\title{
Development of novel Classical and Quantum Information Theory Based Methods for the Detection of Compensatory Mutations in MSAs
}

\author{
Dissertation \\ zur Erlangung des mathematisch-naturwissenschaftlichen Doktorgrades \\ "Doctor rerum naturalium" \\ der Georg-August-Universität Göttingen \\ im Promotionsprogramm PCS \\ der Georg-August University School of Science (GAUSS)
}

\author{
vorgelegt von \\ Mehmet Gültas \\ aus Kirikkale-Türkei
}

Göttingen, 2013 


\section{Betreuungsausschuss}

Professor Dr. Stephan Waack,

Institut für Informatik, Georg-August-Universität Göttingen.

Professor Dr. Carsten Damm,

Institut für Informatik, Georg-August-Universität Göttingen.

Professor Dr. Edgar Wingender,

Institut für Bioinformatik, Universitätsmedizin, Georg-August-Universität Göttingen.

Mitglieder der Prüfungskommission

Referent: $\quad$ Prof. Dr. Stephan Waack, Institut für Informatik, Georg-August-Universität Göttingen.

Korreferent: Prof. Dr. Carsten Damm,

Institut für Informatik, Georg-August-Universität Göttingen.

Korreferent: Prof. Dr. Mario Stanke,

Institut für Mathematik und Informatik, Ernst Moritz Arndt Universität Greifswald

Weitere Mitglieder der Prüfungskommission

Prof. Dr. Edgar Wingender,

Institut für Bioinformatik, Universitätsmedizin, Georg-August-Universität

Göttingen.

Prof. Dr. Burkhard Morgenstern,

Institut für Mikrobiologie und Genetik, Abteilung für Bioinformatik, Georg-August-

Universität Göttingen.

Prof. Dr. Dieter Hogrefe,

Institut für Informatik, Georg-August-Universität Göttingen.

Prof. Dr. Wolfgang May,

Institut für Informatik, Georg-August-Universität Göttingen.

Tag der mündlichen Prüfung: 18. September 2013 


\begin{abstract}
Multiple sequence alignments (MSAs) of homologous proteins are useful tools to characterize compensatory mutations between non-conserved residue sites. The identification of these residue sites in MSAs is an important challenge for understanding the structural basis and molecular mechanism of protein functions. Despite the rich literature on compensatory mutations as well as sequence conservation analysis for the identification of those important residue sites, previous methods often do not take into account biochemical constraints of amino acids which are likely to be crucial for the detection of compensatory mutation signals. However, compensatory mutation signals in MSAs are often masked by noise. Thus, another challenging problem in bioinformatics is the separation of significant signals from the phylogenetic noise and unrelated pair signals.
\end{abstract}

The goal of this thesis is to develop such methods that incorporate biochemical constraints like similarities or dissimilarities of amino acids in identifying compensatory mutations and deal with the noise. Hence, we develop different methods based on classical and quantum information theory and multiple testing procedures.

Our first method is based on classical information theory. It mainly focuses on BLOSUM62dissimilar amino acid pairs as a model of compensatory mutations and incorporates them in the prediction of functionally and/or structurally important sites using a doubly stochastic matrix. To complement this method, we develop our second method applying principles of quantum information theory. The new method differs from the first one by simultaneously modeling similar and dissimilar amino acid pair signals in the compensatory mutation analysis. Moreover, to separate method-based significant compensatory mutation signals from background noise, we develop an MSA-specific statistical model devised for multiple testing problems. By applying this model, we are capable of determining significant signals in MSAs as well as quantifying the error made in terms of the false discovery rate.

To demonstrate the effectiveness of our methods, we evaluate those analyzing important sites of two human proteins, namely epidermal growth factor receptor (EGFR) and glucokinase (GCK). Our results suggest that the MSA-specific statistical model is able to separate significant compensatory mutation signals from the phylogenetic noise and unrelated pair signals. Only considering the dissimilarities of amino acids, the first method successfully deals with disease-associated important sites of both proteins. In contrast, simultaneously focusing on similar and dissimilar amino acid signals, the second method is more sensible to catalytic, allosteric and binding sites. The results further show that overlaps between both methods are quite low, indicating that considerably different sets of residue sites are detected by both methods as functionally and structurally important. As a result of this, we can say that our second method complements the first method when it comes to predicting important sites, rather than replacing it. 



\section{Zusammenfassung}

Multiple Sequenzalignments (MSAs) von homologen Proteinen sind nützliche Werkzeuge, um kompensatorische Mutationen zwischen nicht-konservierten Residuen zu charakterisieren. Die Identifizierung dieser Residuen in MSAs ist eine wichtige Aufgabe um die strukturellen Grundlagen und molekularen Mechanismen von Proteinfunktionen besser zu verstehen. Trotz der vielen Anzahl an Literatur über kompensatorische Mutationen sowie über die Sequenzkonservierungsanalyse für die Erkennung von wichtigen Residuen, haben vorherige Methoden meistens die biochemischen Eigenschaften von Aminosäuren nicht mit in Betracht gezogen, welche allerdings entscheidend für die Erkennung von kompensatorischen Mutationssignalen sein können. Jedoch werden kompensatorische Mutationssignale in MSAs oft durch das Rauschen verfälscht. Aus diesem Grund besteht ein weiteres Problem der Bioinformatik in der Trennung signifikanter Signale vom phylogenetischen Rauschen und beziehungslosen Paarsignalen.

Das Ziel dieser Arbeit besteht darin Methoden zu entwickeln, welche biochemische Eigenschaften wie Ähnlichkeiten und Unähnlichkeiten von Aminosäuren in der Identifizierung von kompensatorischen Mutationen integriert und sich mit dem Rauschen auseinandersetzt. Deshalb entwickeln wir unterschiedliche Methoden basierend auf klassischer- und quantum Informationstheorie sowie multiple Testverfahren.

Unsere erste Methode basiert auf der klassischen Informationstheorie. Diese Methode betrachtet hauptsächlich BLOSUM62-unähnliche Paare von Aminosäuren als ein Modell von kompensatorischen Mutationen und integriert sie in die Identifizierung von wichtigen Residuen. Um diese Methode zu ergänzen, entwickeln wir unsere zweite Methode unter Verwendung der Grundlagen von quantum Informationstheorie. Diese neue Methode unterscheidet sich von der ersten Methode durch gleichzeitige Modellierung ähnlicher und unähnlicher Signale in der kompensatorischen Mutationsanalyse. Des Weiteren, um signifikante Signale vom Rauschen zu trennen, entwickeln wir ein MSA-spezifisch statistisches Modell in Bezug auf multiple Testverfahren.

Wir wenden unsere Methode für zwei menschliche Proteine an, nämlich epidermal growth factor receptor (EGFR) und glucokinase (GCK). Die Ergebnisse zeigen, dass das MSAspezifisch statistische Modell die signifikanten Signale vom phylogenetischen Rauschen und von beziehungslosen Paarsignalen trennen kann. Nur unter Berücksichtigung $B L O$ SUM62-unähnlicher Paare von Aminosäuren identifiziert die erste Methode erfolgreich die krankheits-assoziierten wichtigen Residuen der beiden Proteine. Im Gegensatz dazu, durch die gleichzeitige Modellierung ähnlicher und unähnlicher Signale von Aminosäurepaare ist die zweite Methode sensibler für die Identifizierung von katalytischen und allosterischen Residuen. 



\section{Acknowledgements}

I am indebted to many people for their support and encouragement which was very crucial for the successful completion of my PhD study. In the following lines I would like to use this opportunity to acknowledge and thank all.

First of all, I would like to thank my advisor Prof. Dr. Stephan Waack for giving me the opportunity to join his research group and for his excellent guidance through my study. Without his patient teaching and continuous trust, this work would not have been successful.

My sincere thanks go to my co-advisor Prof. Dr. Carsten Damm patiently guiding me through my work by sharing his experiences with me and for countless hours of fruitful discussions. Without his valuable comments and suggestions, this thesis would not have been possible. I would also like to thank Prof. Dr. Mario Stanke for being in my thesis committee and for sharing valuable ideas.

I would like to thank all my colleagues and friends for their enjoyable and scientifically inspiring environment and for our wonderful friendship: Lennart Obermann, Steffen Herbold, Martin Haubrock, Keyu Wang, Gunnar Krull, Patrick Harms, Thomas Rings, Heike Jachinke, Annette Kadziora, Cansu Topal, Nesrin Tüysüz and Pinar Öz. I would also like to thank the remaining members of my defense committee: Prof. Dr. Edgar Wingender, Prof. Dr. Burkhard Morgenstern, Prof. Dr. Wolfgang May, and Prof. Dr. Dieter Hogrefe.

I want to thank my parents: Elif and Arap Gültas, and my siblings: Dilek Aydogdu, Erdem Gültas and Bayram Gültas for all the love and support over the years.

Especially, I would like to thank my girl friend Susann Michanski who always believes in me and supports me in my research and my daily life. I wouldn't have made it without her. 


\section{Contents}

List of Figures $\quad$ xi

List of Tables $\quad$ xiv

\begin{tabular}{llr}
\hline & Introduction & 2 \\
\hline
\end{tabular}

1.1 Structure of the Thesis $\ldots \ldots \ldots \ldots$

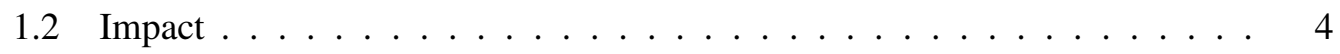

$\begin{array}{lll}2 & \text { Biological Background } & 7\end{array}$

2.1 Amino Acids . . . . . . . . . . . . . . . . . . . . . . 7

2.2 Proteins $\ldots \ldots \ldots \ldots$

2.3 Multiple Sequence Alignments . . . . . . . . . . . . . . . . . . . . . . . . . . . . .

2.4 Mutations in Protein . . . . . . . . . . . . . . . . . . . 12

2.5 Bioinformatics Databases and Tools . . . . . . . . . . . . . . . 13

$2.5 .1 \quad$ Protein Data Bank $\ldots \ldots \ldots \ldots$

2.5 .2 HSSP Database $\ldots \ldots \ldots \ldots \ldots \ldots \ldots$

2.5 .3 Catalytic Site Atlas and PDBsum . . . . . . . . . . . . 21

2.5 .4 Calculation of Distances Between Residues using BioJava . . . . . 21

2.5 .5 BLOSUM Matrices . . . . . . . . . . . . . . . . . . . 23

$\begin{array}{lll}3 & \text { Theoretical Background } & 25\end{array}$

3.1 Classical Information Theory _. . . . . . . . . . . . . . . . 25

3.1 .1 Relative Entropy and Mutual Information . . . . . . . . . . . 28

3.2 Quantum Information Theory $\ldots \ldots \ldots$. . . . . . . . . . . . . 30

$3.2 .1 \quad$ Basic definitions of quantum mechanics . . . . . . . . . . . 30

3.2 .2 Quantum Information and von Neumann Entropy . . . . . . . . . . 36

3.3 Mathematical Foundations . . . . . . . . . . . . . . . . . 37

4 Applying Classical Information Theory for the Compensatory Mutation Analysis 39

4.1 Detecting compensatory mutations by the $\mathbb{U}$-metric . . . . . . . . . . . 39

$4.1 .1 \quad$ Filtering of an MSA . . . . . . . . . . . . . . . . 43

4.2 Determining an MSA-specific lower bound for the significance of U-values 44

4.2 .1 Step 1: Calculation of $p$-values . . . . . . . . . . . . 44 
4.2 .2 Step 2: Setting an MSA-dependent threshold $\tau$. . . . . . . . . 46

4.3 Enhancing prediction by the $\mathbb{U}_{D(\alpha)}$-metric that models dissimilar compensatory mutations $\ldots \ldots \ldots \ldots \ldots \ldots$

$4.3 .1 \quad$ Training data set $\ldots \ldots \ldots \ldots \ldots$

4.3 .2 Preparing substitution matrices $\ldots \ldots \ldots \ldots \ldots$

4.3.3 Creating the Doubly Stochastic Matrix to model dissimilar compen-

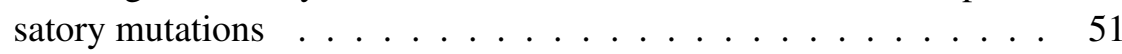

4.3.4 Application of doubly stochastic matrix $D_{\text {CompMut }}$ for the computation of the new $\mathbb{U}_{D(\alpha)}$-metric $\ldots \ldots \ldots \ldots \ldots . \ldots \ldots$



5 Applying quantum information theory for the detection of functionally or structurally important sites 58

5.1 Notation mapping from quantum information theory in protein bioinformatics 58

5.1.1 Calculating the Gram matrices $\mathcal{A}_{\text {ent }}$ and $\mathcal{A}_{\text {sep }}$ to be plugged into

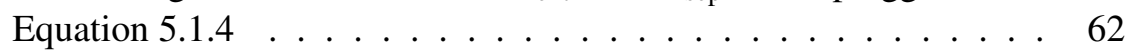

5.1 .2 Simultaneously ensuring Equations $5.1 .3,5.1 .4$ and $5.1 .7 \ldots 63$

5.2 Defining two new metrics $\mathbb{Q}_{\text {ent }}$ and $\mathbb{Q}_{\text {sep }} \ldots \ldots \ldots \ldots 63$

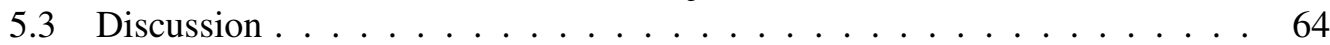

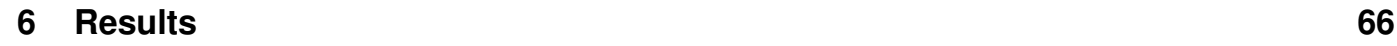

6.1 Essential sites of human EGFR and GCK protein . . . . . . . . . . . . 67

6.1.1 MSAs and 3-D structures of human EGFR and GCK protein . . . . 67

6.2 Applying the $\mathbb{U}$-metric and the $\mathbb{U}_{D(\alpha)}$-metric for the human EGFR and GCK



6.2.1 Position analysis of the Human Epidermal Growth Factor Receptor (EGFR) protein $\ldots \ldots \ldots \ldots \ldots \ldots \ldots 73$

6.2 .2 Position analysis of the Human Glucokinase (GCK) protein . . . . 76

$6.2 .3 \quad$ A comparison between $\mathbb{U}$-metric and $\mathbb{U}_{D(\alpha)}$-metric $\ldots . . . . .79$

6.3 Applying the $\mathbb{Q}_{\text {ent }}$ and the $\mathbb{Q}_{\text {sep }}$-metric for the human EGFR and GCK proteins 80

6.3 .1 Position analysis of the Human EGFR protein . . . . . . . . . 80

6.3 .2 Position analysis of the the Human GCK protein . . . . . . . . . . 85

6.4 A Comparison between CMF-significant sites and QCMF-significant sites. 88

\begin{tabular}{lll}
7 & Discussion & 92 \\
\hline
\end{tabular}

7.1 Connectivity Degree Cut-off $\ldots \ldots$. . . . . . . . . . . . . 92 92

7.2 CMF-significant residue sites . . . . . . . . . . . . . . . . . . 93

$7.3 \quad$ QCMF-significant residue sites . . . . . . . . . . . . . . . . . . . . . . 94

\begin{tabular}{lll}
\hline 8 & Conclusion & 96
\end{tabular}

$8.1 \quad$ Summary . . . . . . . . . . . . . . . . . . . . . 96 
8.2 Outlook . . . . . . . . . . . . . . . . . . . . . . 97

\begin{tabular}{lr}
\hline Bibliography & 99
\end{tabular}

$\begin{array}{llr}9 \text { Appendix } & 110\end{array}$

$9.1 \quad$ Appendix A: Coupled Mutation Finder . . . . . . . . . . . . . . . . . . 110

9.2 Appendix B: Quantum Coupled Mutation Finder . . . . . . . . . . . . . . 123

9.3 Appendix C: PDB enteries of training protein MSAs . . . . . . . . . . . 141

9.4 Appendix D: Curriculum Vitae $\ldots \ldots \ldots \ldots$. . . . . . . . . . . 147 


\section{List of Figures}

2.1 General structure of an amino acid. . . . . . . . . . . . . . . . 7

2.2 Overview of the structural levels in proteins (image from [ [1], p.92]). . 9

2.3 A small section of a multiple sequence alignment. . . . . . . . . . . 11

2.4 The distance calculation: The balls show the $C_{\alpha}$ atoms at positions 14 and 15 in human glucokinase protein with PDB entry 1V4S. Based on the $\mathrm{x}, \mathrm{y}$, and $\mathrm{z}$ coordinates of these atoms, the Euclidean distance is calculated as

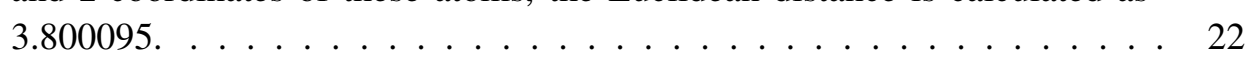

2.5 BLOSUM62 matrix $\ldots \ldots \ldots \ldots \ldots \ldots$

3.1 Venn diagram visualization of entropy $\mathbb{H}(X)$, joint entropy $\mathbb{H}(X, Y)$, conditional entropy $\mathbb{H}(X \mid Y)$, and mutual information $\mathbb{M} \mathbb{I}(X ; Y) . . \ldots . \quad 27$

$4.1 \quad$ A small part of an MSA. The positions $k$ and $l$ show a strong correlation. At position $k$ the $[A$ to $E]$ mutation cause at position $l$ the $[V$ to $G]$ mutation or vice versa. In addition, the position $m$ demonstrates an example for strictly conserved residues. . . . . . . . . . . . . . . . . . 40

4.2 An artificial MSA. The positions $k_{1}$ and $l_{1}$ are slightly conserved and contain only two amino acids. The positions $k_{2}$ and $l_{2}$ contain all 20 amino acids. Since the computation of $\mathbb{M I I}$ values strongly depend on the empirical amino acid distributions of the columns which is also related to the alphabet size, both pairs of columns $\left(k_{1}, l_{1}\right)$ and $\left(k_{2}, l_{2}\right)$ have completely different MII values. The more different amino acids are included in a pair of columns, the higher are their individual column entropy and related joint entropy that often causes a greater $\mathbb{M I I}$ value. However, if a column pair is slightly conserved like $\left(k_{1}, l_{1}\right)$, the alphabet size in these column pair is quite low which results in a less $\mathbb{M I}$ value. $\ldots \ldots . \ldots . \ldots 42$

4.3 p-value distribution of $\mathbb{U}$-values for human GCK protein (PDB Entry 1V4S). The p-values close to zero represent the significant pairs by means of which we assess the individual residue position. In contrast, p-values of unrelated pairs tend to one. As one can see, the p-values of $F_{0}$ distributed U-values are approximately uniform. . . . . . . . . . . . . . 45

4.4 U-value distribution for human GCK protein (PDB Entry 1V4S). The higher the U-value between pairs of columns, the stronger the correlation of amino acids at these columns. . . . . . . . . . . . . . . . . 46 
4.5 p-value distribution of $\mathbb{U}$-values according to the sample mean and variance for human GCK protein (PDB Entry 1V4S). The $p$-values are estimated based on the sample mean and variance which is calculated using all $\mathbb{U}$-values of $M$. As one can see, if we use directly the sample variance for the estimation of shape parameters $\alpha$ and $\beta$ of the $\beta$-distribution, the $p$-values of $\mathbb{U}$-values do not follow the uniform distribution over $[0,1] . \quad \ldots \quad . \quad 47$

4.6 A small part of connectivity degree network of human GCK protein (PDB-Entery 1V4S): The nodes represent individual residue sites and each vertex indicate a significant correlation (U-value) between residue sites $k$ and $l$. The vertex count of a node indicates its connectivity degree. The red circles shows residue sites which have three highest connectivity degree in whole network and thus defined as $(\mathbb{U}, M)$-significant. . . . . . . . . . 50

4.7 Counting pair to pair substitution: In the column pair $(k, l)$ occurs different pair to pair substitution such as $([\mathrm{A}, \mathrm{V}] \leftrightarrow[\mathrm{E}, \mathrm{G}]),([\mathrm{A}, \mathrm{V}] \leftrightarrow[\mathrm{A}, \mathrm{V}])$ and $([E, G] \leftrightarrow[E, G])$ or vice versa. Since the correct order of the sequences in MSAs is unknown, we count the substitutions symmetrically and store the occurrence values of substitutions in a matrix $C . \ldots \ldots \ldots$. . . . . . 52

6.1 The sequence of the human EGFR protein (PDB-Entry 2J6M) (image from $h t t p: / / w w w . r s c b . o r g[2], 05.08 .2013)$. . . . . . . . . . . . . . 68

6.2 The sequence of the human GCK protein (PDB-Entry 1V4S) (image from http://www.rscb.org [2], 05.08.2013). . . . . . . . . . . . . . . 69

6.3 CMF-significant nsSNP positions in human EGFR protein (PDB-Entry 2J6M). The red spheres correspond to structural localization of 15 different CMF-significant nsSNP positions in the EGFR protein. . . . . . . . . . 73

6.4 CMF-significant residue positions are in contact with gefitinib binding sites in human EGFR protein (PDB-Entry 2J6M ). Yellow spheres denote positions of the gefitinib binding sites in the wild type kinase. Blue spheres show the localization of CMF-significant adjacent residue positions which are in contact with these binding sites. Additionally, the CMFsignificant sites 1759, Y764, M766 and K846, shown with green spheres, are already described as nsSNP positions and they are also in contact with gefitinib binding sites E762 and M793, respectively. Circles indicate clusters of gefitinib binding sites and their significant adjacent sites. $\ldots . . .74$

6.5 CMF-significant nsSNP positions in human GCK protein (PDB-Entry 1V4S). Red spheres show the structural localization of 16 different CMFsignificant nsSNP positions in the GCK protein. . . . . . . . . . . . . . 76 
6.6 CMF-significant residue positions are in contact with glucose binding site and allosteric site in human GCK protein (PDB-Entry 1V4S). (A)

\begin{tabular}{|c|}
\hline site and allosteric site in human GCK protein (PDB-Entry 1V4S). (A) \\
\hline \hline Yellow spheres show the structural positions of glucose binding sites (active
\end{tabular}

sites). Blue spheres correspond to localization of CMF-significant adjacent

residue positions which are in contact with these active sites. (B) Orange

spheres denote the allosteric sites. Blue spheres correspond to localization

of just significant adjacent residue positions and green spheres indicate the significant residue positions which are already described as nsSNP positions and in contact with these allosteric sites. Additionally, the significant position R63 is allosteric site by itself and it is also in contact with another allosteric site. Circles indicate clusters of glucose binding sites (A), allosteric sites (B), and their significant adjacent sites. . . . . . . . . . . 77

6.7 p-value distributions of $\mathbb{Q}_{\text {ent }}$ and $\mathbb{Q}_{\text {sep-values for human EGFR protein }}$ (PDB-Entry 2J6M). The blue bars illustrate the $p$-value distribution of the

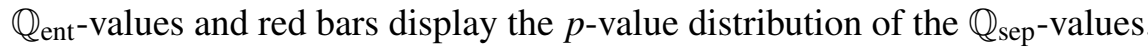

6.8 QCMF-significant residue positions are in contact with catalytic residues in human EGFR protein (PDB-Entry 2J6M). Red spheres denote positions of the catalytic residues. Yellow spheres show the localization of QCMF-significant adjacent residue positions which are in contact with these catalytic residues. Moreover, the QCMF-significant sites A839 and R841 are also catalytic residues by themselves. Green spheres show the structural localization of QCMF-significant nsSNP positions in the EGFR protein. Circles indicate clusters of catalytic residue sites and their significant adjacent sites. . . . . . . . . . . . . . . . 82

6.9 QCMF-significant residue positions are in contact with gefitinib binding sites in human EGFR protein (PDB-Entry 2J6M). Red spheres show the structural localization of the gefitinib binding sites in the wild type kinase. Yellow spheres show significant adjacent residue positions which are in contact with these binding sites. Moreover, the QCMF-significant site T854 is also binding site by itself and interacts with gefitinib binding sites D855. Circles indicate clusters of gefitinib binding sites and their significant

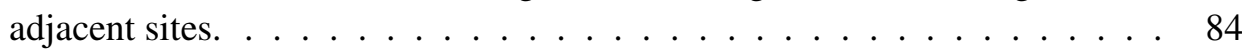

6.10 QCMF-significant positions that are either in contact with allosteric sites or related to nsSNPs in human GCK protein (PDB-Entry 1V4S). Yellow spheres correspond to structural localization of ten QCMFsignificant residue sites which are in contact with allosteric sites where the V62, M210, Y215 are denoted as allosteric sites by themselves and they are also in contact with an other allosteric sites. Green spheres indicate eight QCMF-significant nsSNP positions in the GCK protein. Three of them (T209M, G223S and S453del) are further in contact with allosteric sites M210, I211, V452, V455 and A456. . . . . . . . . . . . 
6.11 QCMF-significant residue positions are in contact with glucose binding site in human GCK protein (PDB-Entry 1V4S). (A) Red spheres show the structural positions of the glucose binding sites (active sites) and yellow spheres show the localization of QCMF-significant adjacent residue positions which are in contact with these active sites. The circles indicate clusters of glucose binding sites and their significant adjacent sites. . . . . . 91 


\section{List of Tables}

2.1 The amino acids: Full names, three-letter abbreviations and single-letter

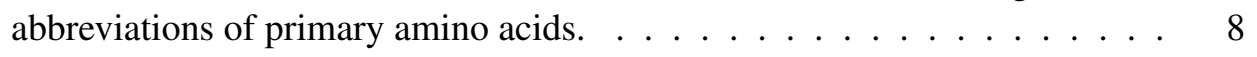

2.2 The standard genetic code: Triplet combinations of nucleotides into amino acids. There are in total of 64 triplets, 61 of them encode the 20 amino acids and 3 of them are stop codons which do not code for any amino



$4.1 \hat{P}_{20 \times 20}$ : Observed probabilities of amino acid pairs in column pair $(k, l)$, where $x_{i}, y_{j} \in 20$ amino acids $(A, C, D, E, F, G, H, I, K, L, M, N, P, Q, R, S, T, V, W, Y) \cdot \ldots \ldots$

$4.2 \hat{P}^{*}{ }_{20 \times 20}$ : Transformed values of joint amino acid pair distribution $\ldots \ldots 55$

$6.1 \quad 43 \mathrm{CMF}-$ significant residue sites found by $\mathbb{U}$ and $\mathbb{U}_{D(\alpha)}$-metric in human EGFR protein. $\ldots \ldots \ldots \ldots \ldots \ldots \ldots \ldots \ldots$

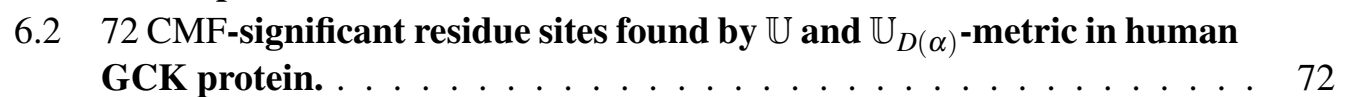

6.3 CMF-significant essential sites in human EGFR protein, which are nearby either nsSNPs or strictly conserved sites. . . . . . . . . . . 75

\begin{tabular}{|ll}
6.4 & CMF-significant essential sites in human GCK protein, which are nearby \\
\hline & either nsSNPs or strictly conserved sites. $\ldots \ldots \ldots \ldots$
\end{tabular}

6.5 Comparison between $\mathbb{U}$-metric and $\mathbb{U}_{D(\alpha)}$-metric $\ldots \ldots \ldots$. . . . . . . . 79

$6.6 \quad 33$ QCMF-significant residue sites found by $\mathbb{Q}_{\text {ent }}$-metric and $\mathbb{Q}_{\text {sep-metric }}$

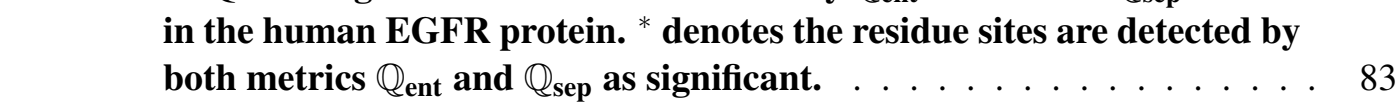

6.7 QCMF-significant essential sites in human EGFR protein, which are nearby either nsSNPs or strictly conserved sites. . . . . . . . . . . . 85

$6.8 \quad 64$ QCMF-significant residue sites found by $\mathbb{Q}_{\text {ent-metric and }} \mathbb{Q}_{\text {sep-metric }}$ in human GCK protein. ${ }^{*}$ denotes the residue sites are detected by both metrics $\mathbb{Q}_{\text {ent }}$ and $\mathbb{Q}_{\text {sep }}$ as significant. . . . . . . . . . . . . . . . . 86

6.9 QCMF-significant essential sites in human GCK protein, which are nearby either nsSNPs or strictly conserved sites. . . . . . . . . . . 88

7.1 Statistical evaluation of CMF-significant residue sites for both human EGFR and GCK protein . . . . . . . . . . . . . . . . 93 
7.2 Statistical evaluation of QCMF-significant residue sites for both human EGFR and GCK proteins. . . . . . . . . . . . . . . . . . . 93

$9.1 \quad$ Pdb enteries of training MSAs. . . . . . . . . . . . . . . . . . . 142 




\section{Introduction}

Multiple sequence alignments (MSAs) of homologous protein sequences give us information about two major features of the proteins of interest. The first one consists of easily detectable highly conserved residue sites that are obviously important for the structure and/or function of the protein while the second one corresponds to compensatory (coupled) mutations between two or more non-conserved residue sites that also contain crucial information on the structural and functional basis of proteins [3]. These compensatory mutations occur according to the functional coupling of mutation positions which might be explained as one mutation in a certain site affecting a compensating mutation at another site, even if both related residue sites are distantly positioned in the protein structure [4-8]. In particular, such mutations at essential residue sites are likely to destroy the protein structure which often results in loss of the protein's function [9, 10]. Thus, for understanding the structural basis and molecular mechanism of protein functions, determination of these compensatory mutated residue sites is as important as strictly conserved sites [4,5,11,12]. These residue sites might be disease-associated, responsible for the maintenance of internal protein volume, or possibly form key sites for interactions within or between proteins [4, 8, 13, 15].

Although strictly conserved residue sites are easily detectable and interpretable in MSAs, the detection of important non-conserved compensatory mutation sites needs more complex approaches. Until now, a variety of studies have employed Pearson's correlation coefficient methods [16-18], perturbation based methods [15, 19] and mutual information (MI) based methods [8, 20-23] because of their simplicity and efficiency. However, these methods strongly depend on the amino acid distributions observed in MSA columns rather than on physical or biochemical similarities or dissimilarites of amino acids that are likely to be crucial for the detection of functionally or structurally important compensatory mutations. In addition, due to background noise, all of these methods interfere with the identification of compensatory mutation signals [9,20,24]. Hence, the significant compensatory mutation signals must be separated from the background noise that might occur as a result of: i) false signals arising from insufficient data; ii) sites with low or high conservation biasing the signal; iii) phylogenetic noise. While the first two types of noise can be easily overcome by appropriately filtering the data [22], phylogenetic noise can only be eliminated to some extent by excluding highly similar sequences from the MSA [9].

Recently, several methods such as bootstrapping, simulation, or randomization methods have been utilized in order to minimize the influence of phylogenetic linkage and stochastic noise [21, 25, 26]. Dunn et al. [9] have introduced the average product correction (APC) 
to adjust MI for background effects. In their study, Merkl and Zwick [22] have used a normalized MI and focused on only the 75 residue pairs with highest normalized MI values as significant for each MSA. Gao et al. [23] have pursued a similar approach, where they have replaced the metric used in [22] with the amino acid background distribution (MIB). While the reduction of background noise in the model of Dunn et al. is not quantified, the approaches of Gao et al. and Merkl and Zwick appear to be over-conservative.

Despite the presence of a variety of different methods as mentioned above, to date, there is still need for a method that contains powerful metrics to take into account biochemical constraints of amino acids and deals with noise and background signals. As a consequence, the main goal of this thesis was to develop classical information theory based methods and quantum information theory based methods to incorporate biochemical similarities or dissimilarities of amino acids in the prediction of functionally or structurally important residue sites. Furthermore, we have developed an MSA-specific statistical model based on multiple testing procedures described in [27,28]. In this way, unlike previous information theory based studies [22, 23], we can separate significant compensatory mutation signals from background noise with respect to our MSA-specific statistical model that quantifies the error made in terms of the false discovery rate.

To demonstrate the performance and functionality of our methods, we analyzed the structurally or functionally important positions of two human proteins, namely epidermal growth factor receptor (EGFR) and glucokinase (GCK). The proteins have been chosen because their important positions experimentally investigated in previous studies [29-40]. As a result, in both proteins our methods detect disease-associated amino acid mutations (nonsynonymous single nucleotide polymorphisms (nsSNPs)), not strictly conserved catalytic or binding sites, and residues that are nearby one of these sites or in the close neighborhood of a strictly conserved positions, which are likely to affect protein stability or functionality [41-43].

\subsection{Structure of the Thesis}

The remainder of this thesis is organized as follows. In Chapter 2, we briefly introduce the descriptions of some biological concepts, databases, and techniques of bioinformatics which are required to motivate, and understand functionally or structurally important amino acids in proteins. In the third chapter, we describe mathematical foundations of classical information theory and quantum information theory, as well as the $\beta$-distribution, upon which we develop our models for the prediction of significant compensatory mutation signals in MSAs. In Chapters 4 and 5, we describe and develop our new classical information theory and quantum information theory based methods and MSA-specific statistical model. In addition, we present the applications of these methods and discuss them in detail at the end of the each chapter. In Chapter 6, we demonstrate the performance and functionality 
of our methods by applying them on the prediction of structurally or functionally important positions of two human proteins. Afterwards, we discuss our results in Chapter 7. In this chapter, we further put our work in the context of a related work which is also developed using classical information theory based method, and compare our results with this related work. Finally, we summarize our conclusions and close by highlighting some potential avenues for further work in Chapter 8.

\subsection{Impact}

We have published the classical information theory based method and the MSA-specific statistical model in a scientific journal article. The quantum information theory based method is also submitted to a scientific journal article and it is currently under review.

\section{Journal Article}

[1] Gültas M, Haubrock M, Tüysüz N, Waack, S: Coupled Mutation Finder: A new entropy-based method quantifying phylogenetic noise for the detection of compensatory mutations. BMC Bioinformatics 2012, 13:225.

[2] Gültas M, Düzgün G, Herzog S, Jäger SJ, Meckbach C, Wingender E, Waack S: Quantum Coupled Mutation Finder: Predicting functionally or structurally important sites in proteins using quantum Jensen-Shannon divergence and CUDA programming. BMC Bioinformatics 2014, 15:96.

\section{Conferences, Workshops, Meetings and Student's thesis}

- Statistical and dynamical models in biology and medicine (October, 2011, Göttingen): Poster presentation

- Workshop über Algorithmen und Komplexität, 63. Theorietag (Januar, 2012, Brandennburg): Oral presentation

- German Conference of Bioinformatics (September, 2012, Jena): Poster presentation

- Meeting Gene Regulation and Information Theory (April, 2013, Halle): Poster presentation

- German Conference of Bioinformatics (September, 2013, Göttingen): Poster presentation

Furthermore, the author identified the topics with Prof. Dr. Stephan Waack for and supervised the following Project Works, Bachelor and Master's thesis.

- Thomas Franke: Identifikation von korrelierten Mutationen auf Basis von H2r, Project Work, 2010 - 2011 
- Thomas Franke: A New Entropy Based Model for the Detection of Correlated Mutations in Multiple Sequence Alignments, Master Thesis, 2011

- Hendrik Kemper: Eine neue Entropie basierte Methode für die Erkennung von kompensatorischen Mutationen: Alternative zu Coupled Mutation Finder, Bachelor Thesis, $2011-2012$

- Linh Dang Truong Khanh: Feature Selection for Compensatory Mutation Analysis in MSAs using Random Forest, Project Work, 2012 - 2013

- Projekt Interdisziplinäres Lernen und Zusammenarbeiten (PILZ): Anwendung der Quanteninformationstheorie für die Identifizierung von kompensatorischen Mutationen, 2012 - 2013

- On going projects

- Linh Dang Truong Khanh: Analysis and prediction of DNA-binding proteins from MSAs using Random Forest, Master Thesis, 2013 -

- Hendrik Kemper: Prediction of functionally important amino acid positions in MSAs using classical and quantum information theory based methods, Master Thesis, 2013 -

- Cornelia Meckbach: Using machine learning methods to combine classical and quantum information theory based metrics for prediction of functionally and/or structurally important amino acid positions in MSAs, Project Work, 2013 - 



\section{Biological Background}

In this chapter, we will give a brief introduction to the key biological concepts and techniques of bioinformatics necessary to motivate, develop, and understand functionally and/or structurally important amino acids in proteins introduced in this thesis. The descriptions in this chapter are based on [1, 44, 47].

\subsection{Amino Acids}

Amino acids are organic molecules which have at least a central carbon atom $\left(C_{\alpha}\right)$ attached to a free carboxyl group $(\mathrm{COOH})$, a free amino group $\left(\mathrm{NH}_{2}\right)$, a hydrogen atom and a side chain group (R) (Figure 2.1). $\mathrm{R}$ is specific to each amino acid and known as residue. The amino acids differ from each other according to the chemical nature of $\mathrm{R}$.



Figure 2.1: General structure of an amino acid.

There are 20 common (or primary) amino acids and each of them is found in proteins, hence they are called proteinogenid 1 amino acids. The names of all amino acids indicate their first isolated source and they are often abbreviated with three-letters and/or one-letter abbreviations (Table 2.1).

\footnotetext{
${ }^{1}$ Proteinogenic means protein building.
} 


\begin{tabular}{lcc}
\hline Full name & Three-letter abbreviation & Single-letter abbreviation \\
\hline Alanine & Ala & $\mathrm{A}$ \\
Cysteine & Cys & $\mathrm{C}$ \\
Aspartic acid & Asp & $\mathrm{D}$ \\
Glutamic acid & Glu & $\mathrm{E}$ \\
Phenylalanine & Phe & $\mathrm{F}$ \\
Glycine & Gly & $\mathrm{G}$ \\
Histidine & His & $\mathrm{H}$ \\
Isoleucine & Ile & $\mathrm{I}$ \\
Lysine & Lys & $\mathrm{K}$ \\
Leucine & Leu & $\mathrm{L}$ \\
Methionine & Met & $\mathrm{M}$ \\
Asparagine & Asn & $\mathrm{N}$ \\
Proline & Pro & $\mathrm{P}$ \\
Glutamine & Gln & $\mathrm{Q}$ \\
Arginine & Arg & $\mathrm{R}$ \\
Serine & Ser & $\mathrm{S}$ \\
Threonine & Thr & $\mathrm{T}$ \\
Valine & Val & V \\
Tryptophan & Trp & W \\
Tyrosine & Tyr & Y \\
\hline
\end{tabular}

Table 2.1: The amino acids: Full names, three-letter abbreviations and single-letter abbreviations of primary amino acids.

All of the proteinogenic amino acids are vital for protein synthesis due to the optimal maintenance of body growth and function. Eleven out of the 20 amino acids: alanine, arginine, asparagine, aspartic acid, cysteine, glutamic acid, glutamine, glycine, proline, serine, and tyrosine are called non-essential amino acids since they are synthesized by the human body. The remaining nine amino acids, histidine, isoleucine, leucine, lysine, methionine, phenylalanine, threonine, tryptophan, and valine are called essential amino acids which cannot be synthesized by the body and thus they must be taken from dietary sources [48].

Amino acids can be classified into hydrophobic and hydrophilic groups based on the physical and chemical characteristics of their residues as follows:

- Hydrophobic groups:

- nonpolar-aliphatic residues: glycine, alanine, proline, valine, leucine, isoleucine, methionine

- aromatic residues: phenylalanine, tyrosine, tryptophan

- Hydrophilic groups:

- polar-charged residues: serine, threonine, cysteine, asparagine, glutamine

- positively charged residues: lysine, arginine, histidine 
- negatively charged residues: aspartate, glutamate

\subsection{Proteins}

Proteins are linear macromolecules made of one or more chains of amino acids arranged in a specific order. Proteins are found in all living cells and are comprised of different combinations of 20 amino acids. The individual side chain of amino acids has a proteinspecific spatial arrangement which defines structure and function of the protein.

According to the structural features, proteins are usually described at four levels of complexity: primary structure, secondary structure, tertiary structure, quaternary structure.

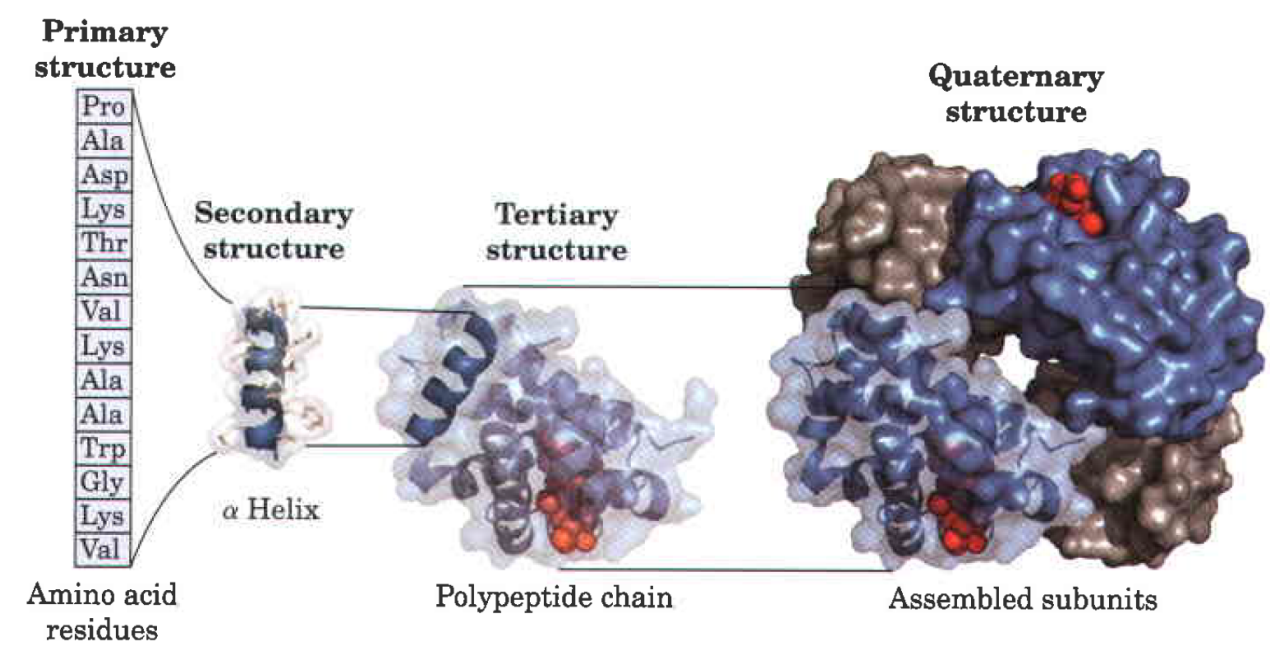

Figure 2.2: Overview of the structural levels in proteins (image from [ [1], p.92]).

Primary Structure: The linear sequence of amino acid residues is referred to as the primary structure of the protein.

Secondary Structure: is the local conformation of the primary structure which is mainly stabilized by hydrogen bonds. There are two types of stable secondary structures: alpha helices $(\alpha$-helices) and beta-sheets ( $\beta$-sheets) that are preferably located at the core of the protein.

Tertiary Structure: is the final three-dimensional (3-D) structure of a protein which consists of secondary structure elements. For stabilization of the protein, very favorable residue interactions occur in this structure. Tertiary structure is unique for each protein and reflects its chemical or structural function. 
Quaternary Structure: is the arrangement of two or more 3-D structures in a protein complex. The quaternary structure is also stabilized by similar interactions as in tertiary structures.

\section{Protein Structure and Function}

Although almost all proteins share the same structural levels, they also contain various chemical environments. Because of this, the behavior of their amino acids can be completely different. The most significant difference occurs between soluble proteins and membrane proteins. While soluble proteins tend to be surrounded by water molecules and have polar or hydrophilic amino acids on their surface, membrane proteins are surrounded by lipids and they contain on their surface the hydrophobic amino acids which are interacting with membrane [49].

The tertiary structure of soluble, membrane, and all other proteins provides essential information for the understanding of their functions. These functions often consist of the reversible binding between protein molecules and other molecules. Such molecules which are usually bound reversibly by proteins are called ligands. Ligands interact with proteins to bind at their specific sites hence they are called binding sites, which are complementary to the ligand size, shape, charge and character [49].

Another important factor for the protein function are enzymes which catalyze the reactions of molecules. After these catalysis, the molecules are called reaction substrates rather than ligands. In addition, ligand binding sites are called catalytic sites or active sites that are crucial for the function and activity of a protein [1,49].

\subsection{Multiple Sequence Alignments}

A multiple sequence alignment (MSA) of proteins contains a set of aligned amino acid sequences in which homologous residues of different sequences are placed in same columns (Figure 2.3). Therefore, functionally or structurally important amino acid positions are often strictly conserved in MSAs [4]. These conserved positions often correspond to catalytic sites, binding sites, sequence family signature, or possibly key sites for interactions within or between proteins. Such conserved positions occur because all living organisms evolved from a common ancestor. According to the common ancestor, it is assumed that all living organism and their proteins are related to each other through evolution [50]. As a result of this, the sequences of proteins are aligned in MSAs very well. In contrast, if the evolutionary relationship between aligned amino acid sequences is distant, their sequences can be aligned poorly. Consequently, for the aligning of these sequences a lot of insertions, deletions, and substitutions (see Section 2.4) are needed in order to create the corresponding MSA. Because of such insertions or deletions, beside of the common 20 amino acid a new element, called gap ('-'), is required for these process. 


Human/1-448
Monkey/14-461
Chimpanzee/14-461
Mouse/14-461
Rat/14-444
Horse/14-461
Cow/3-447
Chicken/14-461
Frog/14-461
Zebrafish/14-461
SeaUrchin/14-444

TLVEQI ALARVDFEF QL QEEDLK TKVEQI ELARGKFEFQL QEEDLK TKVEQI ALA - VDFEFQL QEEDLK EKVEQI EL - - GKFEFYLQEEDLK EKVEQI A - - VD-EFYL QEEDLK HKVEQI EL - - GKFEF ML QEEDLK $H$ - - EQI - - A - GD- DFKLQEADLR HLVEQI AL - - VKQEFI L QEEDLK EKAEQI ELA-GD-EFEL QEEDLA EKVEQI AL - - VKQEFEL QEEDLV EKVEQI ELA - GDQEFEL QEEDLV

Figure 2.3: A small section of a multiple sequence alignment.

A more precisely definition of an MSA is given in [51] as follows;

Definition 2.1 Let $A_{1}, \cdots, A_{r}$ be $r$ sequences over the alphabet of residues $\Sigma$. A multiple sequence alignment (MSA) of A is a matrix which is obtained by inserting gaps ('-') into the original sequences such that all resulting sequences $A_{i}^{*}$ have equal length $L, A_{i}^{*}=A_{i}$ after removal of all gaps from $A_{i}^{*}$, and no column consist of only gaps

$$
A=\left\{\begin{array}{rl}
A_{1}=\left(a_{11}, a_{12}, \ldots, a_{1 n_{1}}\right) \\
A_{2}=\left(a_{21}, a_{22}, \ldots, a_{2 n_{2}}\right) \\
\vdots \\
A_{r}=\left(a_{r 1}, a_{r 2}, \ldots, a_{r n_{r}}\right)
\end{array} \Longrightarrow \quad M S A(A)=\left\{\begin{aligned}
A_{1}^{*}=\left(a_{11}^{*}, a_{12}^{*}, \ldots, a_{1 L}^{*}\right) \\
A_{2}^{*}=\left(a_{21}^{*}, a_{22}^{*}, \ldots, a_{2 L}^{*}\right) \\
\vdots \\
A_{r}^{*}=\left(a_{r 1}^{*}, a_{r 2}^{*}, \ldots, a_{r L}^{*}\right)
\end{aligned}\right.\right.
$$

MSAs are essential and one of the most important computational tools in bioinformatics because they are used almost in every application of bioinformatics, e.g.:

- to visualize and reveal the degree of evolutionary relationship between amino acid sequences,

- to determine the protein family of a newly sequenced protein with unknown structure, function, or evolutionary history in order to get more crucial information about the structure or function of this new protein,

- to predict the secondary structure of proteins, sometimes it even helps for the determination of the 3-D structure,

- to identify functional or structural sites of proteins,

- to identify domains by extracting profiles using them against corresponding databases, 
- to locate DNA regulatory elements such as binding sites,

- to construct a tree for the phylogenetic analysis,

- to cluster proteins according to similar regions.

\subsection{Mutations in Protein}

A protein is a linear sequence over the alphabet of the 20 amino acids that are encoded by the successive triplets of letters from the DNA sequence (Table 2.2). Therefore, mutations in the DNA sequence can lead to variations or substitutions in the structure of corresponding encoded proteins. These variations in the amino acid sequences may lead to a drastic change in the functionality or structural stability of the protein and they can be responsible for diseases. For instance, substitutions at certain positions like $L 858 R, T 790 M$, or $G 719 S$ of the human epidermal growth factor receptor (EGFR) protein result in misregulation of its activity or functionality [4, 52]. EGFR is a famous example in which a small alteration affects protein function, also damages the structural stability of the EGFR protein and increases susceptibility to diseases.

Mutations are generally assigned into three main categories: substitutions, insertions, and deletions. The detailed explanation of these mutations can found in [46] which we highly recommend to interested readers. However, reasons of mutations and how they in detail occur, are out of scope of this thesis, thus we will briefly mention their short definitions.

Substitution: is also known as point mutation, which involve amino acid variations at a certain position. These variations of amino acids in the sequence may produce one of three types of mutation:

1. Missense mutation is also referred as non-synonymous single nucleotide polymorphisms (nsSNPs) that changes one amino acid into another amino acid in proteins. As a result of missense mutation, the amino acid sequence of encoded protein is changed and thus a residue substitution often impacts on the protein structure and also can result in a change or loss of the protein function.

2. Nonsense mutation changes an amino acid into a STOP codon.

3. Frameshift mutation is a genetic mutation caused by a deletion or insertion in a DNA. This mutation causes a change in the reading frame, leading to introduction of unrelated amino acids into the protein, generally followed by a STOP codon.

Deletions: occur if an amino acid is removed from a certain position. As a result, the positions of all the surrounding amino acids are changed.

Insertions: occur if an amino acid is added between two existing ones in sequence which have similar effects like deletions. 


\begin{tabular}{|c|c|c|c|c|c|c|c|c|c|}
\hline & \multicolumn{8}{|c|}{ Second base } & \multirow[b]{2}{*}{ Third base } \\
\hline First base & \multicolumn{2}{|c|}{$\mathbf{T}$} & \multicolumn{2}{|c|}{$\mathbf{C}$} & \multicolumn{2}{|c|}{$\overline{\mathbf{A}}$} & \multicolumn{2}{|c|}{ G } & \\
\hline \multirow{4}{*}{$\mathrm{T}$} & TTT & Phe & TCT & Ser & TAT & Tyr & TGT & Cys & $\mathrm{T}$ \\
\hline & TTC & Phe & $\mathrm{TCC}$ & Ser & TAC & Tyr & TGC & Cys & $\mathrm{C}$ \\
\hline & TTA & Leu & TCA & Ser & TAA & Stop & TGA & Stop & A \\
\hline & TTG & Leu & TCG & Ser & TAG & Stop & TGG & Trp & G \\
\hline \multirow{4}{*}{$\mathrm{C}$} & CTT & Leu & CCT & Pro & CAT & His & CGT & Arg & $\mathrm{T}$ \\
\hline & CTC & Leu & $\mathrm{CCC}$ & Pro & $\mathrm{CAC}$ & His & CGC & Arg & $\mathrm{C}$ \\
\hline & CTA & Leu & $\mathrm{CCA}$ & Pro & CAA & Gln & CGA & Arg & A \\
\hline & CTG & Leu & CCG & Pro & CAG & Gln & CGG & Arg & G \\
\hline \multirow{4}{*}{ A } & ATT & Ile & ACT & Thr & AAT & Asn & AGT & Ser & $\mathrm{T}$ \\
\hline & ATC & Ile & ACC & Thr & $\mathrm{AAC}$ & Asn & AGC & Ser & $\mathrm{C}$ \\
\hline & ATA & Ile & ACA & Thr & AAA & Lys & AGA & Arg & A \\
\hline & ATG & Met & ACG & Thr & $\mathrm{AAG}$ & Lys & AGG & Arg & G \\
\hline \multirow{4}{*}{ G } & GTT & Val & GCT & Ala & GAT & Asp & GGT & Gly & $\mathrm{T}$ \\
\hline & GTC & Val & GCC & Ala & GAC & Asp & GGC & Gly & $\mathrm{C}$ \\
\hline & GTA & Val & GCA & Ala & GAA & Glu & GGA & Gly & A \\
\hline & GTG & Val & GCG & Ala & GAG & Glu & GGG & Gly & G \\
\hline
\end{tabular}

Table 2.2: The standard genetic code: Triplet combinations of nucleotides into amino acids. There are in total of 64 triplets, 61 of them encode the 20 amino acids and 3 of them are stop codons which do not code for any amino acid.

Besides of these three main categories of mutations there is another type of mutation, called correlated mutation, in proteins. Correlated mutations in proteins can be basically explained if one mutation in a certain position occurs within a protein, this mutation can affect a compensating mutation at another position, even if both related residue positions are distantly positioned in the protein structure. For detection of correlated mutations, the basic approach is based on functional coupling of mutation positions in protein multiple sequence alignments which often display correlations between columns [4]. This coupling between residue positions can result from spatial, physical, or chemical restrictions or signaling of allostery [4,7,47]. Thus, determination of these positions is as crucial as the recognition of strictly conserved positions for the understanding the structural basis of protein's function, and for the identification of functionally important residue regions which might be disease associated, responsible for the maintenance of internal protein volume, or possibly form key sites for interactions within or between proteins [4].

\subsection{Bioinformatics Databases and Tools}

In this section, we introduce the databases which are used in this thesis to get 3-D structures of proteins and MSAs. 


\subsubsection{Protein Data Bank}

Protein Data Bank (PDB), at the Research Collaboratory for Structural Bioinformatics (RCSB), is the major database for the structural information of biological macromolecules such as proteins, nucleic acids, or carbohydrates. The PDB database was established by Walter Hamilton at the Brookhaven National Laboratory in 1971 and is freely accessible at http://www.rscb.org. The PDB database contains all publicly available and experimentally determined 3-D structures of approximately 90000 proteins, nucleic acids, protein/nucleic acids complexes, and other biological macromolecules. Although, a large number of database entries are 3-D structures of proteins, the protein structures are often redundant indicating same protein structures which are often observed under different conditions or experiments. In order to present structural information of proteins, PDB has a standard file format in which:

- authors who solved the structure,

- atomic coordinates,

- literature references,

- experimental details about the structure determination,

- primary and secondary structure information such as disulfide bonds, helices, sheets,

- information about binding sites, active sites, as well as hyperlinks to many other scientific databases

are included [2]. In PDB files, each of these information is presented in one line therefore each line of information is called a record. In addition, each PDB file of proteins possesses a name of four characters, known as PDB entry. The PDB entry can consist of either letters A to $\mathrm{Z}$ or digits 0 to 9 like "1V4S.pdb" for the human glucokinase (GCK) protein. 


\subsubsection{PDB file format}

There are several different types of records in PDB files, which are arranged in a specific order to describe a structure. We will briefly describe some of the most important records below. The following descriptions are based on Protein Data Bank Contents Guide [53] where the explanations of all records can be found in detail.

\section{HEADER and TITLE record}

\begin{tabular}{llll|} 
HEADER & TRANSFERASE & 19-NOV-03 & 1V4S \\
TITLE & CRYSTAL &
\end{tabular}

The HEADER record describes the molecule, provides the deposition date of the PDB file and repeats the PDB entry. The TITLE record describes the title of the experiment represented in the entry.

\section{COMPND record}

\begin{tabular}{|ll|}
\hline COMPND & MOL-ID: $1 ;$ \\
COMPND & 2 MOLECULE: GLUCOKINASE ISOFORM 2 \\
COMPND & 3 CHAIN: A; \\
$\cdots$ & $\cdots ;$ \\
\hline
\end{tabular}

The COMPND record contains the description of macromolecular contents of an entry. This record sometimes provides information that may also be found in the TITLE record.

JRNL and REMARK record

\begin{tabular}{|lll|}
\hline JRNL & AUTH & K.KAMATA,M.MITSUYA,T.NISHIMURA,J.EIKI,Y.NAGATA \\
JRNL & TITL & STRUCTURAL BASIS FOR ALLOSTERIC REGULATION OF THE \\
JRNL & TITL 2 & MONOMERIC ALLOSTERIC ENZYME HUMAN GLUCOKINASE \\
JRNL & REF & STRUCTURE V. 12 429 2004 \\
JRNL & REFN & ISSN 0969-2126 \\
JRNL & PMID & 15016359 \\
JRNL & DOI & $10.1016 /$ J.STR.2004.02.005 \\
REMARK & 1 & \\
REMARK & 2 RESOLUTION. & 2.30 ANGSTROMS. \\
REMARK & 3 REFINEMENT. & \\
REMARK & 3 PROGRAM & $:$ CNX 2002 \\
$\cdots$ & $\cdots ;$ & \\
\hline
\end{tabular}

The JRNL record contains the literature reference or publication in which the experiment has been described. However, for more than two references the REMARK record is needed. 
The REMARK record was initially meant for various comments and annotations about the structure of the entry but they are currently used for all general remarks.

\section{HELIX and SHEET record}

The HELIX and SHEET records describe the secondary structure of the protein and polypeptide structures. The HELIX record indicates the location and type (right-handed alpha, etc.) of helices in the molecule. The SHEET record is used to identify the location, sense (anti-parallel, etc.) of the sheet in the molecule.

\begin{tabular}{|llll|}
\hline HELIX & 11 THR A & 14 ALA A & 2118 \\
HELIX & 2 2 GLU A & 22 GLN A & 2453 \\
$\ldots$ & $\cdots ;$ & & \\
SHEET & 1 A 6 LEU A & 58 ARG A & 630 \\
SHEET & 2 A 6 ARG A & 250 ASN A & $254-1$ \\
$\ldots$ & $\ldots$ & & \\
\hline
\end{tabular}

\section{ATOM record}

The ATOM record describes the atomic coordinates containing the $\mathrm{x}, \mathrm{y}, \mathrm{z}$ orthogonal Angstrom ${ }^{2}$ coordinates for atoms in amino acids and nucleic acids.

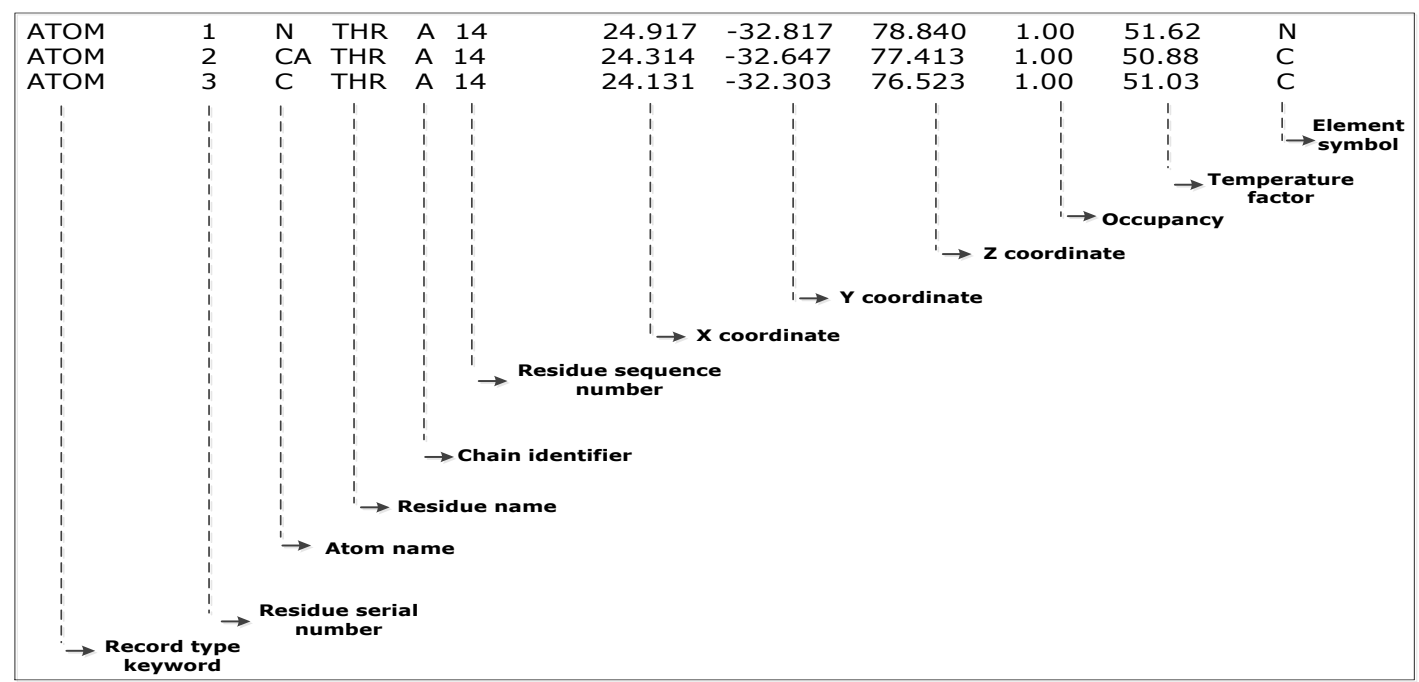

\footnotetext{
${ }^{2} \mathrm{An}$ angstrom is a unit of measurement for very small distances. One Angstrom is equal to $10^{-10}$ meter and it is shown with the symbol of $\AA$.
} 
The third item in the ATOM record shows the atom name that consists of the chemical symbol for the atom type. The atom names begins either with "C", "N", or "O" which indicate carbon, nitrogen, or oxygen atoms, respectively. The next character is the remoteness indicator code which is transliterated according to:

\begin{tabular}{|l|l|l|l|l|l|l|}
\hline$\alpha$ & $\beta$ & $\gamma$ & $\delta$ & $\varepsilon$ & $\eta$ & $\zeta$ \\
$A$ & $B$ & $G$ & $D$ & $E$ & $H$ & $Z$ \\
\hline
\end{tabular}

\subsubsection{HSSP Database}

The HSSP (homology-derived secondary structure of proteins) was created by Chris Sander and Reinhard Schneider which is a derived database to merge primary structure information and secondary/tertiary structure (2-D/3-D) information of proteins [54]. The HSSP database is tightly coupled with the PDB and Swiss-Prot database ${ }^{3}$. While the former database is used to get structural information of proteins, the latter is used to get primary structure information of proteins. According to the structure information, HSSP database provides a special HSPP file like "1V4S.hssp" for each protein from PDB database. The HSSP file contains a multiple sequence alignment of all available homologous that are very likely to share the same 3-D structures [54].

\subsubsection{HSSP file format}

Each HSSP file includes the following four blocks: HEADERS, PROTEINS, ALIGNMENTS, and SEQUENCE PROFILE. While the HEADERS block is obligatory in every HSSP file, the other three blocks are only found if there is at least one homologous alignment. Further, the blocks are separated from one others with the string "\#\#" in HSSP files. Similar to the PDB files, the block information in HSSP files are presented in lines. Below, we will briefly describe these four blocks according to [54].

\section{HEADERS block}

The HEADERS block begins with the version number of HSSP software (program MaxHom) that is used to generate this file. The PDBID (Protein Data Bank Identifier) stands for the PDB entry of the test protein on which the HSSP file is based. The THRESHOLD line describes the homology threshold curve used. The REFERENCE, CONTACT, and DATE lines are already self-explanatory. The header section goes on with information about the PDB lines, including the HEADER, COMPND, SOURCE, and AUTHOR each of them have been explained in the Section 2.5.1. Further, SEQLENGTH, NCHAIN,

\footnotetext{
${ }^{3}$ SWISS-PROT: A protein database that stores only the primary structures of proteins [55].
} 


\begin{tabular}{|ll|}
\hline HSSP & Homology derived secondary structure of proteins, version 2.02011 \\
PDBID & 1 V4S \\
THRESHOLD & according to: $\mathrm{t}(\mathrm{L})=(290.15 * \mathrm{~L} * *-0.562)+5$ \\
REFERENCE & Sander C., Schneider R. : Database of homology-derived protein structures. Proteins \\
CONTACT & Maintained at http://www.cmbi.ru.nl/ by Maarten L. Hekkelman \\
DATE & file generated on 2012-11-25 \\
HEADER & TRANSFERASE 19-NOV-03 1V4S \\
COMPND & MOLECULE: GLUCOKINASE ISOFORM 2 \\
SOURCE & ORGANISM-SCIENTIFIC: HOMO SAPIENS \\
AUTHOR & K.KAMATA,M.MITSUYA,T.NISHIMURA,J.EIKI,Y.NAGATA \\
DBREF & 1V4S A 12 465 UNP P35557 HXK4-HUMAN 13 466 \\
SEQLENGTH & 448 \\
NCHAIN & 1 chain(s) in 1V4S data set \\
NALIGN & 308 \\
NOTATION : & ID: EMBL/SWISSPROT identifier of the aligned (homologous) protein \\
\hline
\end{tabular}

and NALIGN present the length of the sequence, number of distinct chains, and number of aligned sequences in the MSA, respectively. Finally, the NOTATION lines contain some general information about the header description of other blocks and names of the sequence database from which the aligned sequences were obtained, e.g, EMBL/SWISS-PROT or PIR/NBRF.

\section{PROTEINS block}

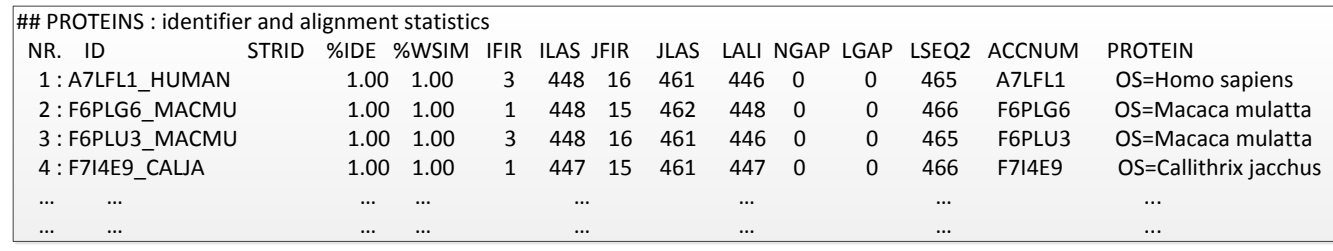

The second block of an HSSP file is the PROTEINS block. This block describes the pairwise aligned data for each protein to the structurally homologous test protein. It begins with "\#\# PROTEINS:". The explanation of headers of columns, which is already described in the NOTATION lines in HEADERS block, is following:

- NR: the line identifier,

- ID: the EMBL/SWISSPROT identifier of the aligned (homologous) protein,

- STRID: the PDB identifier of proteins with known 3-D structure,

- \%IDE: the percentage of the alignment's residue identity, 
- \%SIM (\%WSIM): the (weighted) similarity of the alignment,

- IFIR/ILAS: first and last residue of the alignment in the test sequence,

- JFIR/JLAS: first and last residue of the alignment in the aligned protein,

- LALI: length of the alignment excluding insertions and deletions,

- NGAP: number of insertions and deletions in the alignment,

- LGAP: total length of all insertions and deletions,

- LSEQ2: length of the entire sequence of the aligned protein,

- ACCNUM: Swiss-Prot accession number,

- PROTEIN: one-line description of aligned protein.

\section{ALIGNMENTS block}

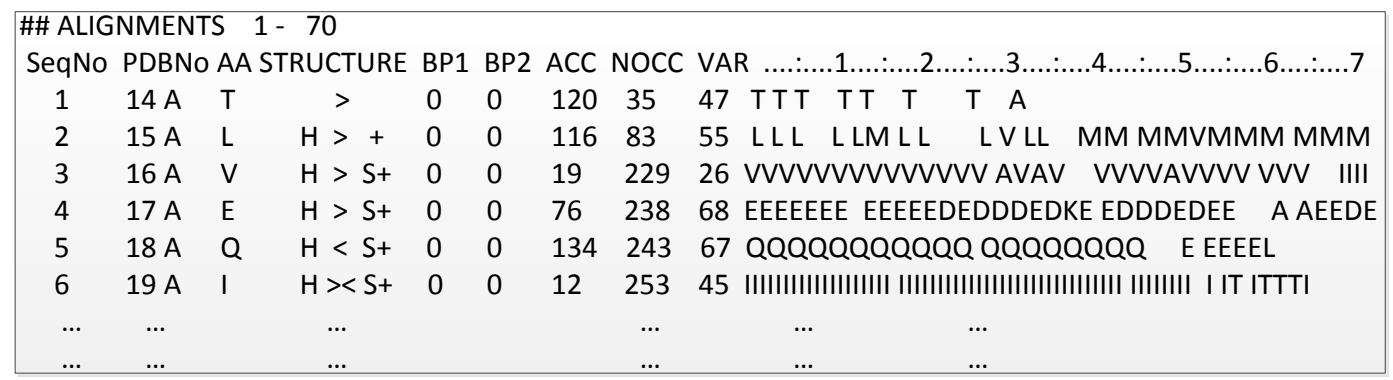

The third section of an HSSP file is the ALIGNMENTS block which begins with "\#\# ALIGNMENTS". In this block, family alignment details are presented residue by residue. The sequences of the test protein and the aligned database proteins are listed vertically, with the leftmost entry at the top. The descriptions of the column headers are explained in the NOTATION lines as following:

- SeqNo: the sequential residue number of the PDB protein as in $\operatorname{DSSP}_{4}^{4}$ file,

- PDBNo: the residue number followed by the name as in the PDB file,

- AA: the amino acid type in the one-letter code,

- STRUCTURE: the summary of the secondary structure as in the DSSP file,

- BP1 and BP2: the $\beta$ bridge partners as obtained from the DSSP file,

- ACC: the surface area of the residue in $\AA^{2}$,

\footnotetext{
${ }^{4}$ DSSP: A database of secondary structure, solvent accessibility and other information derived from 3-D structures in the Protein Data Bank [56].
} 
- NOCC: the number of aligned sequences at this position,

- VAR: the sequence variability derived from the NALIGN alignments.

In this block, while the pair of lower case characters in columns indicates an insertion at corresponding position in the aligned sequence, the dots $(\cdots)$ indicate deletions. In addition, each line of this block contains at most 70 residues of aligned protein sequences. If the total number of aligned sequences (NALIGN, see HEADERS block) is higher than 70 in HSSP files, the ALIGNMENTS block is divided into several intervals like [1 . .70], $[71 \ldots 140]$ etc. until the number of aligned sequences is reached.

\section{SEQUENCE PROFILE block}

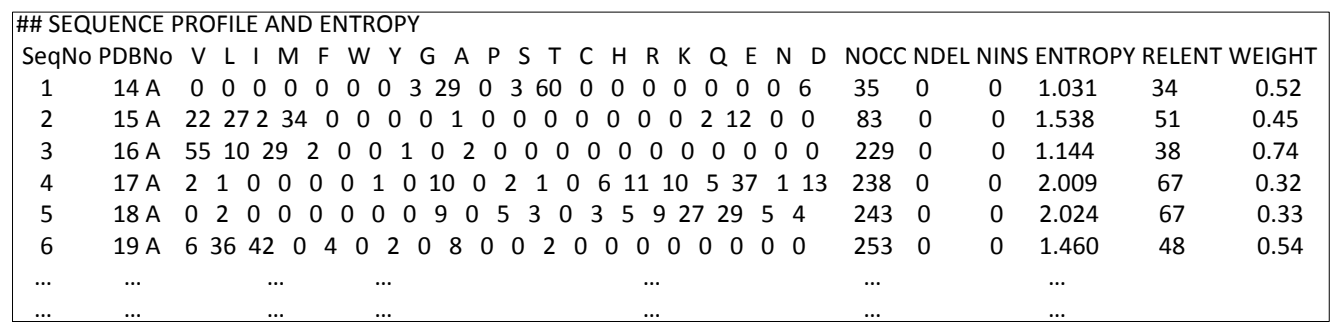

The last section of an HSSP file is the SEQUENCE PROFILE block which begins as usual with "\#\# SEQUENCE PROFILE AND ENTROPY". In this block, the relative frequency of all 20 amino acids at each position is summarized. These frequencies are obtained by counting the amino acids in that position for all aligned sequences, including the test sequence. A frequency of 100 indicates that this position is conserved and consists of only one amino acid. The headers are also explained in the NOTATION lines.

- SeqNo: residue number that is defined in the DSSP file for the test protein (sequence),

- PDBNo: residue number that is defined in the PDB file,

- Columns 3 to 22: the relative frequencies of 20 amino acids under the one letter code,

- NOCC: number of aligned sequences with amino acids in corresponding position (including test sequence),

- NDEL: number of sequences with a deletion, relative to the test protein, at corresponding position,

- NINS: number of sequences with an insertion, relative to the test protein, at corresponding position,

- ENTROPY: measure of sequence variability at corresponding position, 
- RELENT: relative entropy scaled from 0-100,

- WEIGHT: conservation weight.

\subsubsection{HSSP to MSA}

Although an HSSP file contains all of the required information about the protein of interest and its homologous, the format of HSSP files is not suitable to use it directly as an MSA. Therefore, we use the MView algorithm [57] to rewrite an HSSP file in a common MSA file format (fasta format). The MView algorithm is implemented in Perl, Version 5 for UNIX to reformat the results of a sequence database search or a multiple alignment. However, it is important to note that the MView is not a multiple alignment program [57]. For the remainder of this thesis, we use these reformatted MSAs as input for our methods.

\subsubsection{Catalytic Site Atlas and PDBsum}

Catalytic Site Atlas (CSA) is a database which stores experimentally validated catalytic residues in proteins. The database contains two types of validated catalytic residues. While the first one consists of hand-annotated descriptions of catalytic residues, the second type corresponds to equivalent sites in homologous proteins which are found subsequently by a sequence alignment with the original set of hand annotated entries [58]. In the database, there are currently $\sim 6262$ annotated catalytic reside sites according to 968 literature entries. The CSA database is freely available via http://www.ebi.ac.uk/thorntonsrv/databases/CSA/.

Like CSA, the PDBsum database is a part of the European Bioinformatics Institute $(E B I)^{5}$ The PDBsum includes more detailed information about each experimentally determined 3-D structure of proteins in the PDB database. Moreover, the PDBsum illustrates protein structures thereby creates annotated plots for secondary structure of proteins, schematic diagrams of protein-ligand bindings and protein-DNA interactions [59,60]. The PDBsum database is also freely accessible via http://www.ebi.ac.uk/pdbsum/ where a variety of related bionformatics databases are further presented.

In this thesis, we have used both CSA and PDBsum databases in order to observe functionally important catalytic sites and ligand binding sites of proteins which are necessary for the biological evaluation of our methods.

\subsubsection{Calculation of Distances Between Residues using BioJava}

BioJava is one of the most useful open-source bioinformatics library implemented in JAVA. The BioJava library contains a variety of methods and packages each of them are very help-

${ }^{5} \mathrm{EBI}$ is a center for research and services in bioinformatics. 


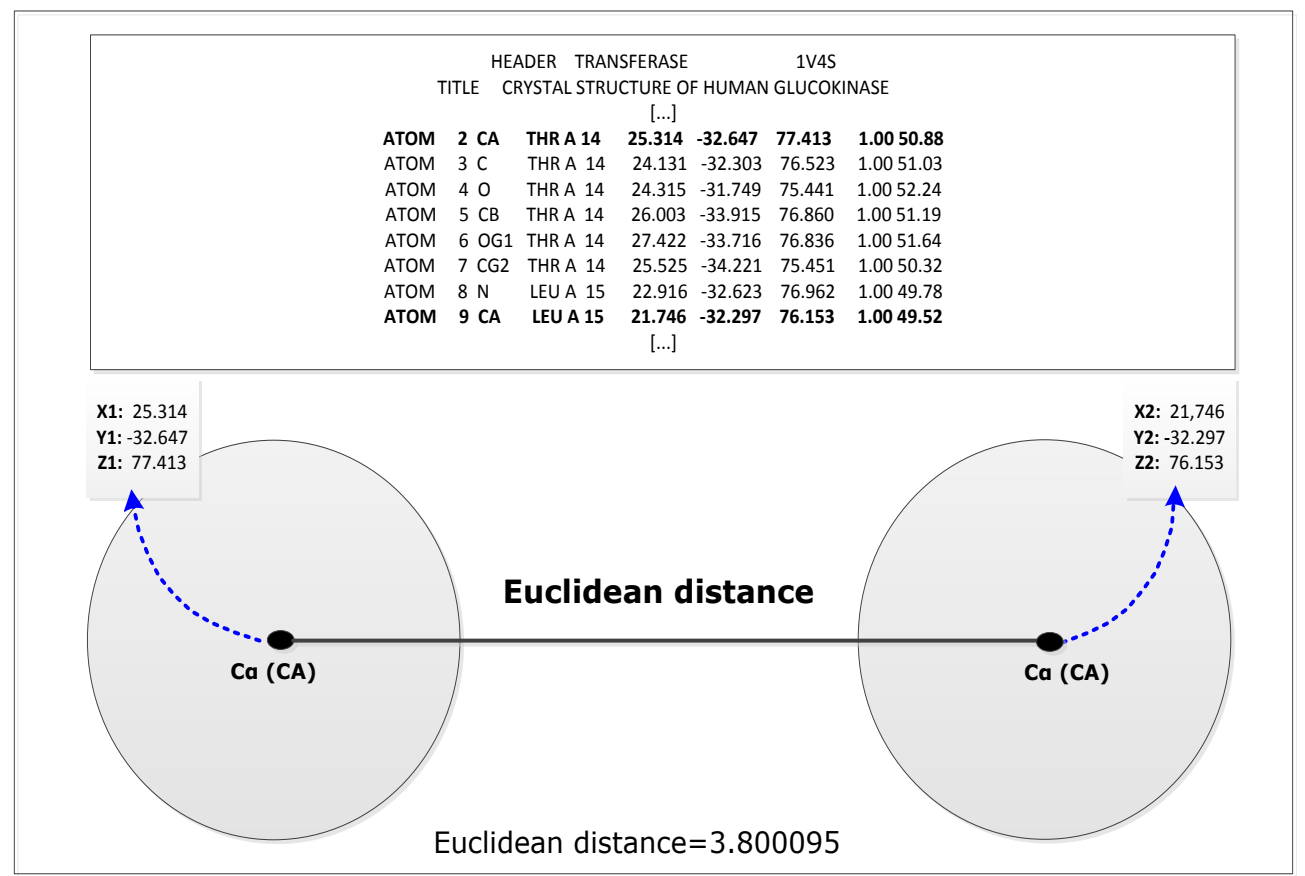

Figure 2.4: The distance calculation: The balls show the $C_{\alpha}$ atoms at positions 14 and 15 in human glucokinase protein with PDB entry $1 \mathrm{~V} 4 \mathrm{~S}$. Based on the $\mathrm{x}, \mathrm{y}$, and $\mathrm{z}$ coordinates of these atoms, the Euclidean distance is calculated as 3.800095 .

ful to process biological data, to parse common file formats, and to manipulate sequences as well as 3-D structures [61].

In this thesis, we have used BioJava for parsing PDB files in order to get three dimensional coordinates of atoms in amino acids. Using these coordinates, we calculate the Euclidean distance between major carbon atoms $\left(C_{\alpha}\right)$ of different amino acids based on the "nearby" definition of Nussinov et al. [62].

Definition 2.2 (Nearby amino acids) Two amino acids (residues) are defined to be in contact or nearby when the distance between their $\left(C_{\alpha}\right)$ atoms is less than $6 \AA$.

It can be assumed that an atom seems likely to be a ball with its three dimensional $\mathrm{x}, \mathrm{y}$, and $\mathrm{z}$ coordinates and according to these coordinates, the Euclidean distance is calculated. 
Definition 2.3 (Euclidean distance) Euclidean distance between two atoms in 3-D space with Cartesian coordinates is defined as

$$
\text { Euclidean distance }=\sqrt{\left(x_{1}-x_{2}\right)^{2}+\left(y_{1}-y_{2}\right)^{2}+\left(z_{1}-z_{2}\right)^{2}},
$$

where $\left(x_{1}, y_{1}, z_{1}\right)$ and $\left(x_{2}, y_{2}, z_{2}\right)$ are the $x, y$ and $z$ coordinates of both atoms under study [63].

Figure 2.4 shows the distance calculation between two $C_{\alpha}$ atoms in different amino acids of human GCK protein. However, it is important to note that in this distance calculation we do not consider the van der Waals radius 6

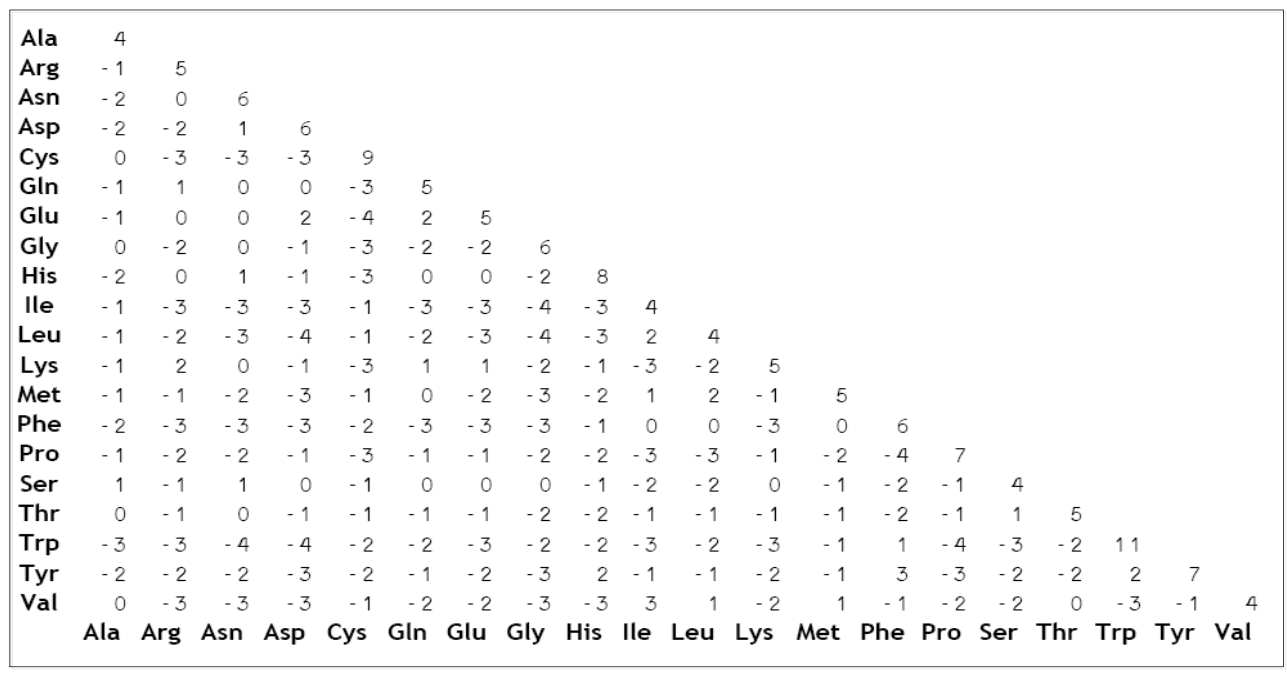

Figure 2.5: BLOSUM62 matrix

\subsubsection{BLOSUM Matrices}

The BLOSUM (BLOcks SUbstitution Matrix) is a widely used scoring matrix for sequence alignments of proteins which were first introduced by Henikoff et. al in 1992 [64]. In order to construct BLOSUM matrices, Henikoff et al. have used the BLOCKS Database [65] which contains a set of aligned and un-gapped regions from protein families. In BLOSUM

\footnotetext{
${ }^{6}$ van der Waals radius: indicates the radius of an imaginary hard sphere around an atom that is used for spatial measurements between atoms.
} 
matrices, each substitution between amino acids is assigned a score according to their observed frequencies in the data set of homologous sequences of proteins. The substitution scores can be negative, zero, or positive. A positive score indicates that the occurrence of substitutions between similar or identical amino acids is greater than that would have been observed by random chance in the data set. In contrast, a negative score indicates that the occurrence of substitutions between dissimilar amino acids is less than that would have been observed by random chance. A score of zero means that the occurrence of weakly similar amino acids is equal to that expected by chance.

The BLOSUM matrices are $20 \times 20$ symmetric matrices and its columns and rows are indexed by the elements of amino acids (see Figure 2.5). Further, each BLOSUM matrix is denoted by BLOSUMr, where $0<r<100$, like BLOSUM50 or BLOSUM62 which indicates the percent identity level of sequences in the alignment. For example, for BLOSUM50, the sequences with approximately $50 \%$ identity were counted. 


\section{Theoretical Background}

In this chapter, we provide a basic introduction to information theory. In the first section we start with the definitions of entropy, mutual information, Kullback Leibler divergence and Jensen-Shannon divergence. Most of this section is based on Chapter 2 in [66] which provides a friendly introduction to the concept of entropy. In the next section, we present some of the fundamental principles of quantum mechanics which are further required to explain the definitions of the quantum information theory. The introduced definitions and notations in this section are based on [67--71].

\subsection{Classical Information Theory}

The Shannon entropy is the key concept of the classical information theory and it is a measure of the average uncertainty of a random variable. Assume that we have a random variable $X$. The entropy of $X$ quantifies the amount of information that we gain after we learn the value of $X$. Alternatively, it can be explained that the entropy of $X$ provides a measure for the amount of uncertainty about $X$ before we learn its value.

Definition 3.1 (Entropy) $\quad$ Let $X$ be a discrete random variable with alphabet $\mathfrak{X}$ and probability distribution $p(x)=\operatorname{Pr}\{X=x\}, x \in \mathfrak{X}$, where probabilities satisfy $0 \leq p(x) \leq 1$ and $\sum_{x \in \mathfrak{X}} p(x)=1$. The entropy of $X$ is denoted by $\mathbb{H}(X)$ and defined as

$$
\mathbb{H}(X)=-\sum_{x \in \mathfrak{X}} p(x) \log p(x) .
$$

In this definition, the $\log (x)$ indicates the $\log$ in base two. Further, we adopt the convention that $p(x) \log p(x)=0$, if $p(x)=0$ for realizations with zero probability. In the Equation 3.1.1, one can easily see that the computation of $H(X)$ only depends on the probability distribution of $X$ and it takes its maximum value when all probabilities $p\left(x_{i}\right)$ are equal.

Definition 3.2 (Joint Entropy) Let $X$ and $Y$ two discrete random variables with alphabet $x \in \mathfrak{X}$ and $y \in \mathfrak{Y}$. Further, let $p(x)=\operatorname{Pr}\{X=x\}$ and $p(y)=\operatorname{Pr}\{Y=y\}$ be their individual probability distributions. The joint entropy $\mathbb{H}(X, Y)$ of $X$ and $Y$ with a joint probability distribution $p(x, y)$ is defined as

$$
\mathbb{H}(X, Y)=-\sum_{x} \sum_{y} p(x, y) \log p(x, y) .
$$


Definition 3.3 (Conditional Entropy) Let $X$ and $Y$ be discrete random variables with joint probability distribution $p(x, y)$ and conditional distribution $p(x \mid y)$, then the conditional entropy $\mathbb{H}(X \mid Y)$ is defined as

$$
\mathbb{H}(X \mid Y=y)=-\sum_{x} p(x \mid y) \log p(x \mid y) .
$$

The conditional entropy $\mathbb{H}(X \mid Y)$ (Equation 3.1.3) can be written as

$$
\begin{aligned}
\mathbb{H}(X \mid Y) & =\sum_{y} p(y) \mathbb{H}(X \mid Y=y) \\
& =-\sum_{y} p(y) \sum_{x} p(x \mid y) \log p(x \mid y) \\
& =-\sum_{x, y} p(x, y) \log p(x \mid y) .
\end{aligned}
$$

If $X$ and $Y$ are independent then

$$
\mathbb{H}(X \mid Y)=\mathbb{H}(X)
$$

It is important to note that the conditional entropy is not symmetric, i.e., $\exists X, Y$ such that $\mathbb{H}(X \mid Y) \neq \mathbb{H}(Y \mid X)$.

Theorem 3.1 (Chain rule for entropy:) The joint entropy $\mathbb{H}(X, Y)$ of a pair of discrete random variables $X$ and $Y$ can be defined according to $\mathbb{H}(X), \mathbb{H}(Y), \mathbb{H}(X \mid Y)$, and $\mathbb{H}(Y \mid X)$ as follows

$$
\mathbb{H}(X, Y)=\mathbb{H}(X)+H(Y \mid X) \text { or } \mathbb{H}(Y, X)=\mathbb{H}(Y)+\mathbb{H}(X \mid Y) .
$$

The additive property of Shannon entropy can be applied as

$$
\mathbb{H}(X, Y)=\mathbb{H}(X)+\mathbb{H}(Y),
$$

if $X$ and $Y$ are completely independent.

According to the Equation 3.1.2, we can show that $\mathbb{H}(X, Y)=\mathbb{H}(Y, X)$, i.e.,

$$
\mathbb{H}(X, Y)=\mathbb{H}(X)+\mathbb{H}(Y \mid X)=\mathbb{H}(Y)+\mathbb{H}(X \mid Y)=\mathbb{H}(Y, X),
$$

from which the following equation is obtained as

$$
\mathbb{H}(X)-\mathbb{H}(X \mid Y)=\mathbb{H}(Y)-\mathbb{H}(Y \mid X) .
$$

The Equation 3.1.4 is different but equivalent forms of mutual information which we will introduce in the next section. The venn diagram in Figure 3.1 illustrates the relationships between $\mathbb{H}(X), \mathbb{H}(X), \mathbb{H}(X, Y), \mathbb{H}(X \mid Y)$ and $\mathbb{H}(Y \mid X)$. 


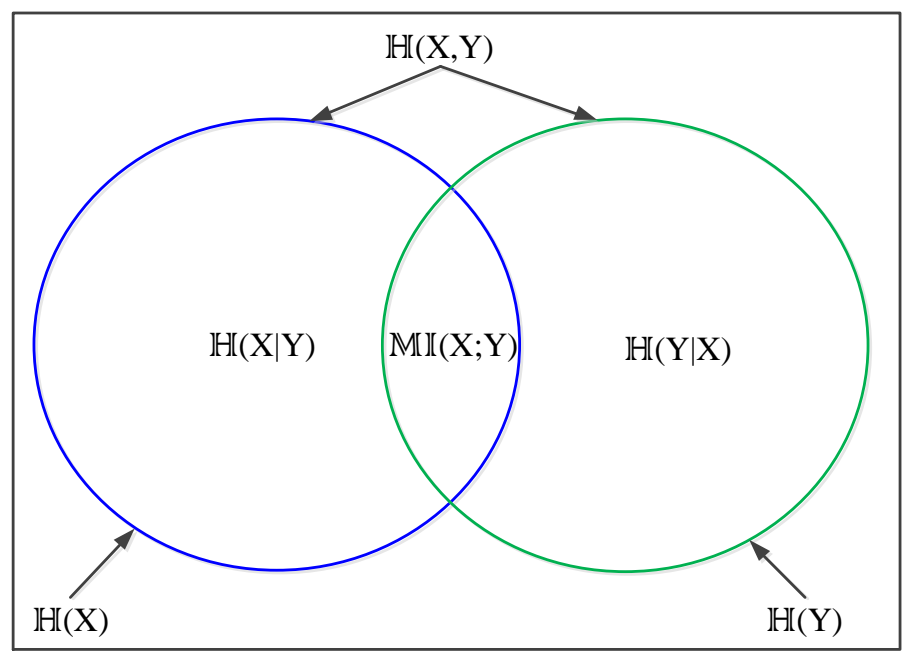

Figure 3.1: Venn diagram visualization of entropy $\mathbb{H}(X)$, joint entropy $\mathbb{H}(X, Y)$, conditional entropy $\mathbb{H}(X \mid Y)$, and mutual information $\mathbb{M I}(X ; Y)$.

\section{Theorem 3.2 (Basic properties of Shannon entropy)}

1. Non-negativity: The entropy is always non-negative for any $p(x)$ :

$$
\mathbb{H}(X) \geq 0 .
$$

2. Upper bound: The maximum value of $\mathbb{H}(X)$ for random variable $X$ with alphabet size $n$ is $\log (n)$ :

$$
\mathbb{H}(X) \leq \log (n) .
$$

3. Conditioning reduces the entropy:

$$
\mathbb{H}(X) \geq \mathbb{H}(X \mid Y) .
$$

4. Subadditivity:

$$
\mathbb{H}(X, Y) \leq \mathbb{H}(X)+\mathbb{H}(Y) .
$$

The equality is only possible if and only if the random variables $X$ and $Y$ are independent.

5. $\mathbb{H}(X) \leq \mathbb{H}(X, Y)$, the equality is only possible if and only if the random variable $Y$ is a function of $X$. 
6. The entropy $\mathbb{H}(X)$ is concave in the probability density function $p(x)$.

\subsubsection{Relative Entropy and Mutual Information}

The relative entropy is another entropic measure that quantifies the difference between two probability distributions over the same alphabet.

Definition 3.4 (Kullback Leibler divergence) Relative entropy or Kullback Leibler (KL) divergence between two probability distributions $p(x)$ and $q(x)$ with alphabet $\mathfrak{X}$ is defined as

$$
K L(p \| q)=\sum_{x} p(x) \log \frac{p(x)}{q(x)}=\mathbb{H}(X)-\sum_{x} p(x) \log q(x) .
$$

In the Equation 3.1.5, we adopt the convention that $0 \log 0=0$ and $-p(x) \log 0=\infty$ if $p(x)>0$.

\section{Theorem 3.3 (Basic properties of the Kullback Leibler divergence)}

1. Non-negativity: $K L(p \| q) \geq 0$.

2. $K L(p \| q)=0$ if and only if $p(x)=q(x)$.

3. The $K L$ divergence is not symmetric: $K L(p \| q) \neq K L(q \| p)$ if $p(x) \neq q(x)$.

4. The KL divergence does not satisfy the triangle inequality.

Definition 3.5 (Jensen-Shannon divergence) Jensen-Shannon (JS) divergence is a symmetrized, smoothed, and bounded version of the KL divergence between two (or more) probability distributions. The JS divergence between two probability distributions $p(x)$ and $q(x)$ with weights $\pi_{1}, \pi_{2}$ defined by $[72]$ and $[73]$ independently as

$$
J S(p(x) \| q(x))=\pi_{1} K L\left(p(x) \| \frac{p(x)+q(x)}{2}\right)+\pi_{2} K L\left(q(x) \| \frac{p(x)+q(x)}{2}\right),
$$

where $\pi_{1}$ and $\pi_{2}$ satisfy the constraints $\pi_{1}+\pi_{2}=1$, and $0 \leq \pi_{i} \leq 1$.

The $J S$ divergence can also be written in the following form

$$
J S(p \| q)=\mathbb{H}\left(\frac{p+q}{2}\right)-\frac{1}{2} \mathbb{H}(p)-\frac{1}{2} \mathbb{H}(q)
$$

where $\mathbb{H}(p)=-\sum_{i} p_{i} \log p_{i}$ is the Shannon entropy and $\pi_{1}=\pi_{2}=\frac{1}{2}$.

Theorem 3.4 (Basic properties of Jensen-Shannon divergence)

1. Non-negativity: $J S(p \| q) \geq 0$. 
2. $J S(p \| q)=0$ if and only if $p=q$.

3. The JS divergence is symmetric and always well defined: $J S(p \| q)=J S(q \| p)$.

4. The JS divergence is bounded $0 \leq J S(p \| q) \leq 1$.

5. The square root of the JS divergence satisfy the triangle inequality but JS divergence does not.

6. The JS divergence can be generalized in order to quantify the difference between more than two probability distributions $p_{1}, p_{2}, \ldots, p_{m}$ with weights $\pi_{1}, \pi_{2}, \ldots, \pi_{m}$ by

$$
J S\left(p_{1}, p_{2}, \ldots, p_{m}\right)=\mathbb{H}\left[\sum_{i=1}^{m} \pi_{i} p_{i}\right]-\left[\sum_{i=1}^{m} \pi_{i} \mathbb{H}\left[p_{i}\right]\right],
$$

where $\sum_{i=1}^{m} \pi_{i}=1$.

Definition 3.6 (Mutual Information) Let $X$ and $Y$ be two random variables with a joint probability distribution $p(x, y)$ and marginal probability distributions $p(x)$ and $p(y)$. The mutual information $\mathbb{M I}(X ; Y)$ between $X$ and $Y$ is defined as

$$
\mathbb{M I}(X ; Y)=\sum_{x} \sum_{y} p(x, y) \log \frac{p(x, y)}{p(x) p(y)} .
$$

Alternatively, the $\mathbb{M I}(X ; Y)$ between $X$ and $Y$ can be written according to $\mathbb{H}(X), \mathbb{H}(Y)$ and $\mathbb{H}(X, Y)$ as

$$
\mathbb{M I}(X ; Y)=\mathbb{H}(X)+\mathbb{H}(Y)-\mathbb{H}(X, Y) .
$$

The mutual information $\mathbb{M I}(X ; Y)$ between two random variables $X$ and $Y$ is the reduction in the uncertainty of $Y$ due to the knowledge of $X$ (or vice versa).

\section{Theorem 3.5 (Basic properties of mutual information)}

1. Non-negativity: $\mathbb{M I}(X ; Y) \geq 0$.

2. $\mathbb{M I}(X ; Y)=0$ if and only if $X$ and $Y$ are independent.

3. $\mathbb{M I}(X ; Y)$ is symmetric: $\mathbb{M I}(X ; Y)=\mathbb{M I}(Y ; X)$.

4. $\mathbb{M I}(X ; Y)=\mathbb{H}(X)-\mathbb{H}(X \mid Y)$.

5. $\mathbb{M I}(X ; Y) \leq \mathbb{H}(X)$. 


\subsection{Quantum Information Theory}

Before we begin to define quantum information theory, we firstly have to introduce some of the basic principles and notations of quantum mechanics, which are necessary to explain and to understand quantum information theory. The definitions and notations in this section are based on [67.-71]. Moreover, we do not consider in this thesis complex vector spaces and only deal with real ones.

\subsubsection{Basic definitions of quantum mechanics}

\section{Quantum bits}

In computer science, the elementary unit of information is represented by a bit which is the fundamental concept of classical computation and classical information. A bit describes the unit information of a classical system being in one of two possible states, in either the state 0 or the state 1 . Like a classical bit, a quantum bit or short qubit is the elementary unit of information describing two-state system in the quantum information theory. Two possible states for a qubit are $|0\rangle$ and $|1\rangle$ which are equivalent to the states of a classical bit. The notation, "|.)", called as Dirac notation, is the standard notation of quantum mechanics. Although classical bits and qubits look very similar at first glance, there exists a fundamental difference between them. While a classical bit can be in either the state 0 or 1 , a qubit can also be in the state superposition which is a linear combination of the states $|0\rangle$ and $|1\rangle$,

$$
|\psi\rangle=\alpha|0\rangle+\beta|1\rangle .
$$

Equation 3.2.1 shows that the $|\psi\rangle$ is in a superposition where $\alpha$ and $\beta$ are coefficients with unit norm

$$
|\alpha|^{2}+|\beta|^{2}=1 .
$$

The coefficients $\alpha$ and $\beta$ are referred to as quantum probability amplitudes and their square magnitudes, $|\alpha|^{2}$ and $|\beta|^{2}$, indicate the probability of $|\psi\rangle$ for being in states $|0\rangle$ and $|1\rangle$, respectively.

\section{Dirac notation}

In quantum mechanics, Dirac notation is a very useful way of expressing unit-length vectors that are used to represent the states of a physical system. Further, the Dirac notation is especially nice to use because it provides a very helpful way of specifying vector and matrix operations of quantum states. The notation consist of bras "L.|" and kets "|.|" therefore it is also called as "braket $(\langle. \mid\rangle$.$) notation". Basically, a ket |$.$\rangle indicates in this thesis a$ column vector of a real column vector space $\mathfrak{A}$ and a bra $\langle$.$| is obtained by transposing a$ ket |.). In quantum information theory it is usual to consider complex spaces. But for our purposes it suffices to deal with real ones. The multiplication of bras by kets results in the 
brakets notation "|.|. $\rangle$ " which is also referred as standard scalar product or inner product on $\mathfrak{A}$. Suppose we have the vectors $|u\rangle$ and $|v\rangle$. Their inner product, $\langle u \mid v\rangle$ has the following geometric interpretation. It is equal to $\|u\|\|v\| \cos \alpha$, where $\alpha$ is the angle between the vectors $|u\rangle$ and $|v\rangle$, and $\|u\|=\sqrt{\langle u \mid u\rangle}$ and $\|v\|=\sqrt{\langle v \mid v\rangle}$ are the Euclidean norms (lengths) of $|u\rangle$ and $|v\rangle$.

The column vector representation of $|0\rangle$ and $|1\rangle$ is denoted as

$$
\begin{aligned}
& |0\rangle=\left(\begin{array}{l}
1 \\
0
\end{array}\right), \\
& |1\rangle=\left(\begin{array}{l}
0 \\
1
\end{array}\right) .
\end{aligned}
$$

The bras corresponding to the kets $|0\rangle$ and $|1\rangle$ can be shown as

$$
\begin{aligned}
& \langle 0|=\left(\begin{array}{ll}
1 & 0
\end{array}\right), \\
& \langle 1|=\left(\begin{array}{ll}
0 & 1
\end{array}\right) .
\end{aligned}
$$

Although a bra does not represent quantum states, it is required for performing calculations like probability amplitudes in the quantum theory. Recall the qubit in the Equation 3.2.1, in order to determine the probability of $|\psi\rangle$ for being in state $|0\rangle$, we will combine the state of the $|\psi\rangle$ with the bra $\langle 0|$ in the following calculation as

$$
\begin{aligned}
\langle 0 \mid \psi\rangle & =\langle 0|(\alpha|0\rangle+\beta|1\rangle) \\
& =\alpha\langle 0|| 0\rangle+\beta\langle 0|| 1\rangle \\
& =\alpha\left(\begin{array}{ll}
1 & 0
\end{array}\right)\left(\begin{array}{l}
1 \\
0
\end{array}\right)+\beta\left(\begin{array}{ll}
0 & 1
\end{array}\right)\left(\begin{array}{l}
0 \\
1
\end{array}\right) \\
& =\alpha \cdot 1+\beta \cdot 0 \\
& =\alpha .
\end{aligned}
$$

Likewise, calculating the quantity $\langle 1 \mid \psi\rangle$ we can determine the probability of $|\psi\rangle$ for being in states $|1\rangle$. Further, we can also calculate the quantities $\langle 0 \mid 1\rangle$ and $\langle 1 \mid 0\rangle$ as

$$
\begin{aligned}
& \langle 0 \mid 1\rangle=0, \\
& \langle 1 \mid 0\rangle=0,
\end{aligned}
$$

which show the probability of $|0\rangle$ for being in state $|1\rangle$ and the probability of $|1\rangle$ for being in state $|0\rangle$. Since the vectors $|0\rangle$ and $|1\rangle$ are orthogonal to each other and have no overlap, their inner products are equal to zero. In contrast to this, the inner products $\langle 0 \mid 0\rangle$ and $\langle 1 \mid 1\rangle$ 
are equal to one,

$$
\begin{aligned}
& \langle 0 \mid 0\rangle=1, \\
& \langle 1 \mid 1\rangle=1 .
\end{aligned}
$$

\section{Outer product}

In contrast to the inner product, the product of a ket $|u\rangle$ with a bra $\langle v|$ results in a matrix. It is defined as the outer product which is used to represent the density operator of a system.

Let $|u\rangle$ and $|v\rangle$ be two qubits given by

$$
|u\rangle=\left(\begin{array}{c}
a_{0} \\
a_{1}
\end{array}\right) \text { and, }|v\rangle=\left(\begin{array}{c}
b_{0} \\
b_{1}
\end{array}\right)
$$

The outer product is an expression like $|u\rangle\langle v|$ and calculated by

$$
|u\rangle\langle v|=\left(\begin{array}{c}
a_{0} \\
a_{1}
\end{array}\right)\left(b_{0}, b_{1}\right)=\left(\begin{array}{cc}
a_{0} b_{0} & a_{0} b_{1} \\
a_{1} b_{0} & a_{1} b_{1}
\end{array}\right) .
$$

We can perform the bras $\langle$.$| and kets |$.$\rangle to represent this matrix in Dirac notation as$

$$
|u\rangle\left\langle v\left|=a_{0} b_{0}\right| 0\right\rangle\left\langle 0\left|+a_{0} b_{1}\right| 0\right\rangle\left\langle 1\left|+a_{1} b_{0}\right| 1\right\rangle\left\langle 0\left|+a_{1} b_{1}\right| 1\right\rangle\langle 1| .
$$

Recall the qubit in the Equation 3.2.1, combining $|\psi\rangle=\alpha|0\rangle+\beta|1\rangle$ with the matrix $|u\rangle\langle v|$, we determine the effect of this matrix on $|\psi\rangle$ as

$$
(|u\rangle\langle v|)(|\psi\rangle)=|u\rangle\langle v \mid \psi\rangle=\langle v \mid \psi\rangle|u\rangle .
$$

This means that $|u\rangle\langle v|$ is a projection of $\mathfrak{A}$ onto the subspace spanned by $|u\rangle$. Every linear operator on $\mathfrak{A}$ can be represented by a linear combination of such products. The transpose $A^{T}$ of a linear operator $A=\sum_{i j} a_{i j}\left|u_{i}\right\rangle\left\langle v_{j}\right|$ equals $\sum_{i j} a_{i j}\left|v_{j}\right\rangle\left\langle u_{i}\right|$.

\section{Tensor product}

The tensor product is a fundamental mathematical operation of combining vector spaces to form a new larger vector space. Suppose we have two two-dimensional vectors:

$$
|u\rangle=\left(\begin{array}{l}
a_{1} \\
a_{2}
\end{array}\right) \text {, and }|v\rangle=\left(\begin{array}{l}
b_{1} \\
b_{2}
\end{array}\right)
$$

The tensor product of $|u\rangle \otimes|v\rangle$ is 


$$
|u\rangle \otimes|v\rangle=|u v\rangle=\left(\begin{array}{l}
a_{1} \\
a_{2}
\end{array}\right) \otimes\left(\begin{array}{l}
b_{1} \\
b_{2}
\end{array}\right)=\left(\begin{array}{l}
a_{1} b_{1} \\
a_{1} b_{2} \\
a_{2} b_{1} \\
a_{2} b_{2}
\end{array}\right) .
$$

We can also calculate the tensor product of qubit states $|0\rangle$ and $|1\rangle$ using their vector representations:

$$
|00\rangle=\left(\begin{array}{l}
1 \\
0 \\
0 \\
0
\end{array}\right),|01\rangle=\left(\begin{array}{l}
0 \\
1 \\
0 \\
0
\end{array}\right),|10\rangle=\left(\begin{array}{l}
0 \\
0 \\
1 \\
0
\end{array}\right),|11\rangle=\left(\begin{array}{l}
0 \\
0 \\
0 \\
1
\end{array}\right) .
$$

Likewise, we can derive the tensor product of two matrices that is referred to as the Kronecker product. Suppose we have two operators $U=\left|u_{1}\right\rangle\left\langle u_{2}\right|$ and $V=\left|v_{1}\right\rangle\left\langle v_{2}\right|$ and their matrix representations are given by

$$
U=\left|u_{1}\right\rangle\left\langle u_{2}\right|=\left(\begin{array}{ll}
a_{11} & a_{12} \\
a_{21} & a_{22}
\end{array}\right) \text { and } \quad V=\left|v_{1}\right\rangle\left\langle v_{2}\right|=\left(\begin{array}{ll}
b_{11} & b_{12} \\
b_{21} & b_{22}
\end{array}\right) .
$$

The matrix representation of the tensor product $U \otimes V$ can be written as

$$
\left|u_{1}\right\rangle\left\langle u_{2}|\otimes| v_{1}\right\rangle\left\langle v_{2}|=| u_{1}\right\rangle\left\langle v_{1}|\otimes| u_{2}\right\rangle\left\langle v_{2}\right|=U \otimes V,
$$

and calculated as

$$
U \otimes V=\left(\begin{array}{ll}
a_{11} V & a_{12} V \\
a_{21} V & a_{22} V
\end{array}\right)=\left(\begin{array}{llll}
a_{11} b_{11} & a_{11} b_{12} & a_{12} b_{11} & a_{12} b_{12} \\
a_{11} b_{21} & a_{11} b_{22} & a_{12} b_{21} & a_{12} b_{22} \\
a_{21} b_{11} & a_{21} b_{12} & a_{22} b_{11} & a_{22} b_{12} \\
a_{21} b_{21} & a_{21} b_{22} & a_{22} b_{21} & a_{22} b_{22}
\end{array}\right) .
$$

In general, if $\left|u_{1}\right\rangle,\left|u_{2}\right\rangle \in \mathfrak{A}_{1}$ and $\left|v_{1}\right\rangle,\left|v_{2}\right\rangle \in \mathfrak{A}_{2}$ are states, then $\left|u_{1} v_{1}\right\rangle=\left|u_{1}\right\rangle \otimes\left|v_{1}\right\rangle \in \mathfrak{A}_{1} \otimes$ $\mathfrak{A}_{2}$ and $\left|u_{2} v_{2}\right\rangle=\left|u_{2}\right\rangle \otimes\left|v_{2}\right\rangle \in \mathfrak{A}_{1} \otimes \mathfrak{A}_{2}$ are tensor products of column vectors. The tensor products of row vectors $\left\langle u_{1} v_{1}\right|=\left\langle u_{1}\right| \otimes\left\langle v_{1}\right|$ and $\left\langle u_{2} v_{2}\right|=\left\langle u_{2}\right| \otimes\left\langle v_{2}\right|$ are the transposes of $\left|u_{1} v_{1}\right\rangle$ and $\left|u_{2} v_{2}\right\rangle$, where $\left\langle u_{1} v_{1} \mid u_{2} v_{2}\right\rangle=\left\langle u_{1} \mid v_{2}\right\rangle\left\langle u_{1} \mid v_{2}\right\rangle$.

\section{The Density operator or matrix}

The traditional quantum mechanics distinguishes between pure states and mixed states: pure state of a quantum system is a vector $(|\rangle$.$) with a unit length in a vector space (the quantum$ states which we have considered until now are also pure states); mixed state is a statistical mixture of two or more states. While the pure states are represented by state vectors, mixed 
states are described by density matrices. A density matrix $\rho$ is an operator representing a quantum state that describes a part of the composite system.

Suppose we have the following two state vectors:

$$
\begin{aligned}
& |u\rangle=\alpha|x\rangle+\beta|y\rangle, \\
& |v\rangle=\gamma|x\rangle+\delta|y\rangle .
\end{aligned}
$$

The density matrix for each of these states is the outer product of the state vector with itself:

$$
\begin{aligned}
\rho_{u} & =|u\rangle\langle u|, \\
\rho_{v} & =|v\rangle\langle v| .
\end{aligned}
$$

Now, we can generalize the density matrix for $n$ possible states. Suppose we have $n$ states and a quantum system is in one of these states $\left|\psi_{i}\right\rangle$ where $i=1,2, \ldots, n$ with respective probabilities $p_{i}$. The notation $\left\{p_{i},\left|\psi_{i}\right\rangle\right\}$ is called an ensemble of pure states. Then the density matrix for the entire system is defined as

$$
\rho=\sum_{i=1}^{n} p_{i}\left|\psi_{i}\right\rangle\left\langle\psi_{i}\right|
$$

Definition 3.7 (Eigenvalues and Eigenstates of the density matrix) Given a density matrix $\rho$ on a vector space, a non-zero state vector $|\psi\rangle$ is defined as eigenstate of $\rho$ if the following equation is satisfied

$$
\rho|\psi\rangle=\lambda|\psi\rangle
$$

where $\lambda$ is called an eigenvalue of $\rho$.

In order to determine eigenvalues of a density matrix $\rho$, the characteristic equation $\operatorname{det} \mid \rho-$ $\lambda I \mid=0$ is used, where det indicates the determinant of the matrix $\rho-\lambda I$ and $I$ denotes the identity matrix. The values of $\lambda$ are the eigenvalues which represent the solutions of this characteristic equation.

Theorem 3.6 (Key properties of a Density operator) An operator $\rho$ is called as a density operator if and only if it satisfies the following properties:

1. The density operator $\rho$ is Hermitian 7 .

$$
\rho^{\dagger}=\left(\sum_{i} p_{i}\left|\psi_{i}\right\rangle\left\langle\psi_{i}\right|\right)^{\dagger}=\sum_{i} p_{i}\left|\psi_{i}\right\rangle\left\langle\psi_{i}\right|=\rho
$$

\footnotetext{
${ }^{7}$ A Hermitian matrix is a matrix which is equal to its own transpose.
} 
2. $\rho$ is positive semi-definite for any state vector $|\psi\rangle$ :

$$
\langle\psi|\rho| \psi\rangle \geq 0 .
$$

3. $\rho$ has unit trace: $\operatorname{Tr}(\rho)=1$.

\section{Entangled and Separable States}

One of the most striking features of quantum mechanics is the fact that the systems can become entangled. Suppose we have two systems $A$ and $B$ and their composite state is given by a density matrix $\rho_{A B}$. We define these two systems to be entangled if they have interacted sometime in the past and we cannot construct the individual systems again now. According to this interaction, the values of certain properties of system $A$ are correlated with the values that those properties will assume for system $B$ [71]. In contrast, we say that two system are separable if they are not entangled indicating that the corresponding composite state $\rho_{A B}$ can be broken into tensor product of these two systems.

More precisely, suppose we have two density operators as

$$
\rho_{A}=\sum_{i, j=1}^{n} \alpha_{i j}|i\rangle\langle j| \text { and } \rho_{B}=\sum_{k, l=1}^{n} \beta_{k l}|k\rangle\langle l| .
$$

Their tensor product

$$
\rho_{A} \otimes \rho_{B}=\sum_{i, j, k, l=1}^{n} \alpha_{i j} \beta_{k l}|i k\rangle\langle j l|
$$

is a separable density operator since it is the probability distributions over products of the kind

$$
\sum_{m} p_{m} \rho_{A, m} \otimes \rho_{B, m}\left(p_{m}>0, \sum_{m} p_{m}=1\right)
$$

\section{The reduced density operator: Partial Trace}

Suppose we have two systems $A$ and $B$ and their composite state is given by a density operator $\rho_{A B}$ as

$$
\rho_{A B}=\sum_{i, j, k, l=1}^{n} \gamma_{i k j l}|i k\rangle\langle j l|
$$


We define the reduced density operator of the system $A$ and $B$ as

$$
\begin{aligned}
& \rho_{A}=\operatorname{tr}_{B}\left(\rho_{A B}\right)=\operatorname{tr}_{B}\left(\rho_{A} \otimes \rho_{B}\right), \\
& \rho_{B}=\operatorname{tr}_{A}\left(\rho_{A B}\right)=\operatorname{tr}_{A}\left(\rho_{A} \otimes \rho_{B}\right),
\end{aligned}
$$

where $t r_{A}$ and $t r_{B}$ are the partial traces over system $A$ and $B$, respectively. The partial traces $t r_{A}$ and $t r_{B}$ are defined by

$$
\operatorname{tr}_{A}(\rho)=\sum_{k, l=1}^{n}\left(\sum_{i=1}^{20} \gamma_{i k i l}\right)|k\rangle\left\langle l\left|, \quad \operatorname{tr}_{B}(\rho)=\sum_{i . j=1}^{n}\left(\sum_{k=1}^{20} \gamma_{i k j k}\right)\right| i\right\rangle\langle j| .
$$

It is important to note that partial traces of density operators are also density operators.

\subsubsection{Quantum Information and von Neumann Entropy}

In this section, we will consider the basis of quantum information theory. We will begin by defining the von Neumann Entropy of a density operator $\rho$ that is the quantum analogue of the Shannon entropy $\mathbb{H}$. After that, we will go on with quantum definitions of relative entropy, joint entropy, mutual information, and Jensen-Shannon divergence [67--71].

Definition 3.8 (Quantum Entropy: von Neumann Entropy) Let $\rho$ be a density operator on a $n$-dimensional space. The von Neumann entropy of $\rho$ is denoted by $V N E(\rho)$ and defined as

$$
V N E(\rho)=-\operatorname{tr}(\rho \log \rho)=-\sum_{i=1}^{n} \lambda_{i} \log \lambda_{i}
$$

where $\lambda_{1}, \ldots, \lambda_{n}$ are eigenvalues of $\rho$ and we define $0 \log 0=0$.

Definition 3.9 (Joint Quantum Entropy) Let A, B be two subsystems and $A B$ be their composite system. Let $\rho_{A B}, \rho_{A}, \rho_{B}$ be corresponding density operators of the system and its subsystems. Quantum joint entropy is defined as

$$
\operatorname{VNE}\left(\rho_{A B}\right)=-\operatorname{tr}\left(\rho_{A B} \log \rho_{A B}\right) .
$$

Definition 3.10 (Quantum Mutual Information) The quantum mutual information of a composite system $A B$ defined as

$$
V N E\left(\rho_{A} ; \rho_{B}\right)=V N E\left(\rho_{A}\right)+V N E\left(\rho_{B}\right)-V N E\left(\rho_{A B}\right) .
$$

\section{Theorem 3.7 (Basic properties of quantum entropy)}

1. Non-negativity: The quantum entropy is non-negative for any density operator $\rho_{A}$ :

$$
\operatorname{VNE}\left(\rho_{A}\right) \geq 0,
$$


where the equality is only possible if and only if $\rho_{A}$ is a pure state.

2. Upper bound: The maximum value of $\operatorname{VNE}\left(\rho_{A}\right)$ for a density operator $\rho_{A}$ is $\log (n)$, where $n$ is the dimension of the system:

$$
V N E\left(\rho_{A}\right) \leq \log (n)
$$

3. If a composite system $\rho_{A B}$ is in a pure state:

$$
\operatorname{VNE}\left(\rho_{A}\right)=\operatorname{VNE}\left(\rho_{B}\right)
$$

4. Subadditivity:

$$
\operatorname{VNE}\left(\rho_{A B}\right) \leq \operatorname{VNE}\left(\rho_{A}\right)+\operatorname{VNE}\left(\rho_{B}\right) .
$$

The equality is only possible if the composite system $\rho_{A B}$ equal to the tensor product of two subsystems

$$
V N E\left(\rho_{A} \otimes \rho_{B}\right)=V N E\left(\rho_{A}\right)+V N E\left(\rho_{B}\right),
$$

which corresponds to two subsystems with uncorrelated information.

Definition 3.11 (Quantum Relative entropy) The quantum relative entropy between two density operators $\rho$ and $\sigma$ is defined, analogously to the corresponding classical quantity, as

$$
R V N E(\rho \| \sigma)=\operatorname{tr}(\rho \log \rho)-\operatorname{tr}(\rho \log \sigma) .
$$

Definition 3.12 (Quantum Jensen-Shannon divergence) Similar to the classical JensenShannon divergence, the quantum Jensen-Shannon divergence $(Q J S D(\rho \| \sigma))$ between two density operators $\rho$ and $\sigma$ is a symmetrized and smoothed version of quantum relative entropy. The $(Q J S D(\rho \| \sigma))$ is defined as

$$
\operatorname{QJSD}(\rho \| \sigma)=\frac{1}{2}\left[R V N E\left(\rho \| \frac{\rho+\sigma}{2}\right)+R V N E\left(\sigma \| \frac{\rho+\sigma}{2}\right)\right] .
$$

The Equation 3.2.34 can be rewritten in terms of the von Neumann entropy as

$$
\operatorname{QJSD}(\rho \| \sigma)=\operatorname{VNE}\left(\frac{\rho+\sigma}{2}\right)-\frac{1}{2} \operatorname{VNE}(\rho)-\frac{1}{2} \operatorname{VNE}(\sigma) .
$$

\subsection{Mathematical Foundations}

In following we briefly introduce the definition of the $\beta$-distribution and its parameter estimation which is necessarily for the development of our significant model. The descriptions in this section are based on [74]. 
Definition 3.13 ( $\beta$-distribution) A continuous random variable $X$ has a $\beta$-distribution over the interval $[0,1]$ with positive shape parameters $\alpha$ and $\beta$ if its density function is defined as

$$
f_{X}(x)=\frac{1}{B(\alpha, \beta)} x^{(\alpha-1)(1-\beta)^{(\beta-1)}}, \quad 0 \leq x \leq 1 .
$$

In Equation 3.3.1 $B(.,$.$) is the \beta$-function and it is calculated as

$$
B(\alpha, \beta)=\int_{0}^{1} t^{\alpha-1}(1-t)^{\beta-1} d t, \quad \alpha>0 \text { and } \beta>0 .
$$

The mean $\mu$ and variance $\sigma^{2}$ of the $\beta$-distribution are

$$
\begin{aligned}
\mu & =\frac{\alpha}{\alpha+\beta}, \\
\sigma^{2} & =\frac{\alpha \beta}{(\alpha+\beta)^{2}(\alpha+\beta+1)} .
\end{aligned}
$$

In addtion, the cumulative distribution function of $\beta$-distribution is given as

$$
F_{X}(x)=\frac{1}{B(\alpha, \beta)}=\int_{0}^{x} t^{\alpha-1}(1-t)^{\beta-1} d t, \quad 0 \leq x \leq 1 \text { and } \alpha, \beta>0 .
$$

For a given sample mean $\hat{\mu}$ and variance $\hat{\sigma}$ of finite sample of size $N$, the shape parameters $\alpha$ and $\beta$ of a beta distribution can be estimated as

$$
\begin{aligned}
& \hat{\alpha}=\hat{\mu}\left(\frac{\hat{\mu}(1-\hat{\mu})}{\hat{\sigma}}-1\right), \\
& \hat{\beta}=(1-\hat{\mu})\left(\frac{\hat{\mu}(1-\hat{\mu})}{\hat{\sigma}}-1\right),
\end{aligned}
$$

where $\hat{\mu}$ and $\hat{\sigma}$ can be calculated using following equations as

$$
\begin{aligned}
& \hat{\mu}=\frac{1}{N} \sum_{i=1}^{N} x_{i}, \\
& \hat{\sigma}=\frac{1}{N} \sum_{i=1}^{N}\left(x_{i}-\hat{\mu}\right)^{2} .
\end{aligned}
$$




\section{Applying Classical Information Theory for the Compensatory Mutation Analysis}

In this chapter, we will present two entropy-based metrics, namely $\mathbb{U}$-metric and $\mathbb{U}_{D(\alpha)^{-}}$ metric, for the detection of compensatory mutations in MSAs. While the former one was developed by Merkl et. al in [22], the latter has been developed during this thesis in order to complete the U-metric. Further, we will present a new MSA-specific statistical model based on multiple testing procedures in order to determine significant $\mathbb{U}$ and $\mathbb{U}_{D(\alpha)}$-values, respectively.

We previously published an important part of this chapter in [4] (see Appendix A 9.1). The descriptions of both the $\mathbb{U}$-metric and the $\mathbb{U}_{D(\alpha)}$-metric as well as the new MSA-specific statistical model approach are based on this publication.

\subsection{Detecting compensatory mutations by the $\mathbb{U}$-metric}

Let $M$ be an arbitrarily chosen but fixed MSA for the protein under investigation. In order to determine the correlation between columns of $M$, Merkl et. al. used in [22] a normalized measure of mutual information ranging over the interval $[0,1]$. It is denoted as $\mathbb{U}$-metric and defined as

$$
\mathbb{U}(k, l)=2 \cdot \frac{\mathbb{H}(k)+\mathbb{H}(l)-\mathbb{H}(k, l)}{\mathbb{H}(k)+\mathbb{H}(l)}=2 \cdot \frac{\mathbb{M I}(k ; l)}{\mathbb{H}(k)+\mathbb{H}(l)},
$$

where $\mathbb{H}(k)$ and $\mathbb{H}(l)$ are the entropy of the empirical amino acid distributions of the columns $k$ and $l$ under study, and $\mathbb{H}(k, l)$ is their joint entropies. The empirical distributions of amino acids are observed based on the occurrence of each amino acid in columns $k$ and $l$ (see Figure 4.1). Likewise, extending the concept of empirical amino acid distributions, we can observe the empirical joint distributions of amino acid pairs in the column pair $(k, l)$.

It is important to note that during the observation of these amino acid distributions we only take into account the standard 20 amino acids and do not consider the gaps ('-') as a real component of the protein. Hence, we exclude them when we determine distributions of amino acids in columns or in column pairs. 


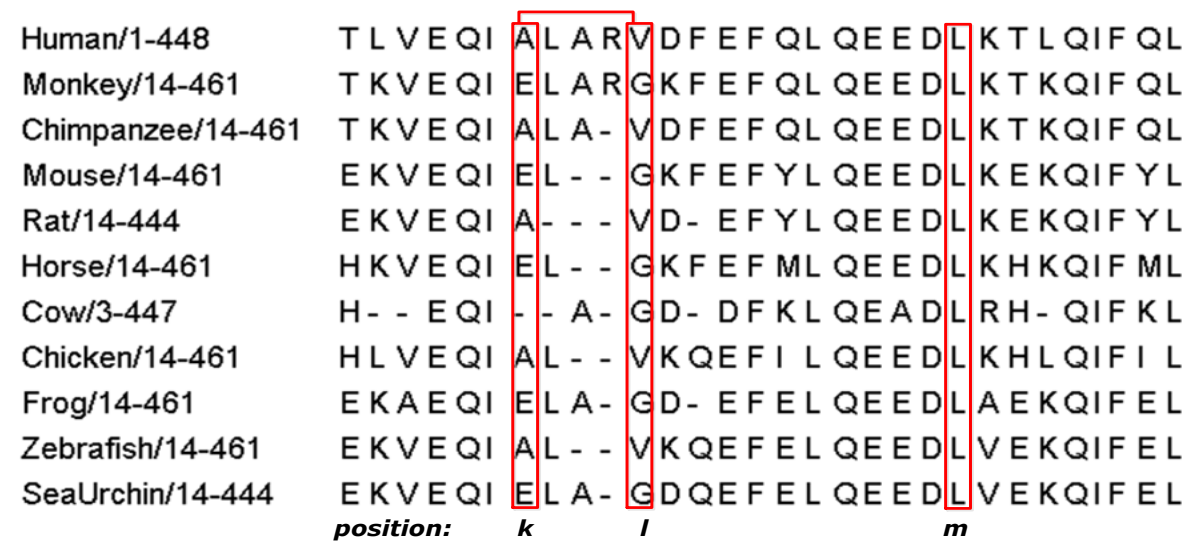

Figure 4.1: A small part of an MSA. The positions $k$ and $l$ show a strong correlation. At position $k$ the $[A$ to $E]$ mutation cause at position $l$ the $[V$ to $G]$ mutation or vice versa. In addition, the position $m$ demonstrates an example for strictly conserved residues.

As mentioned above, the $\mathbb{U}(k, l)$-value of a column pair $(k, l)$ ranges from 0 to 1 . According to the Figure 3.1, one can easily see that if the columns $k$ and $l$ are completely dependent, the individual column entropies are equal to their corresponding joint entropy, i.e.,

$$
\mathbb{H}(k)=\mathbb{H}(l)=\mathbb{H}(k, l) .
$$

In this case, we have $\mathbb{U}(k, l)=1$, which is the upper bound of the $\mathbb{U}$-metric. On the other hand, if both columns are completely independent, we can see according to the Figure 3.1 that the sum of individual column entropies corresponds to the their joint entropy, i.e.,

$$
\mathbb{H}(k, l)=\mathbb{H}(k)+\mathbb{H}(l),
$$

where $\mathbb{U}(k, l)=0$, which is the lower bound of the $\mathbb{U}$-metric.

\section{Calculation of the column entropies}

Since we utilize the concept of information theory, it is necessary to consider amino acids as a set of a random variable in columns of MSAs. Like in Figure 4.1, let $k$ and $l$ be two columns and $n$ is the number of sequences in the MSA $M$ under study. Further, we assume that $X$ and $Y$ are two random variables with same alphabet $\mathfrak{X}$, where the alphabet size corresponds to 20 amino acids (throughout the remainder of this thesis unless otherwise noted, the alphabet size is always 20). The random variables $X$ and $Y$ characterize the frequencies of amino acids in given columns $k$ and $l$ in $M$, respectively. 
According to this, we can write associated empirical amino acid distributions of each columns as $\hat{p}(x)=\operatorname{Pr}\{X=x\}, \hat{p}(y)=\operatorname{Pr}\{Y=y\}, x, y \in \mathfrak{X}$. Further, the empirical joint amino acid pair distribution is denoted by $\hat{p}\left(x_{i}, y_{i}\right)=\hat{p}\{X=x, Y=y\}$. Then, the marginal distributions $\hat{p}\left(x_{i}\right), \hat{p}\left(y_{i}\right)$ and joint distribution $\hat{p}\left(x_{i}, y_{i}\right)$ are calculated as

$$
\begin{array}{r}
\hat{p}\left(x_{i}\right)=\frac{\#\left(x_{i}\right)}{n}, \\
\hat{p}\left(y_{i}\right)=\frac{\#\left(y_{i}\right)}{n}, \\
\hat{p}\left(x_{i}, y_{i}\right)=\frac{\#\left(x_{i}, y_{i}\right)}{n},
\end{array}
$$

where \# $\left(x_{i}\right), \#\left(y_{i}\right)$, and \# $\left(x_{i}, y_{i}\right)$ are observed frequencies of amino acids $\left(x_{i}\right),\left(y_{i}\right)$ and pair of amino acids $\left(x_{i}, y_{i}\right)$ in the given columns $k$ and $l$ and in column pairs $(k, l)$.

Using the Equations 4.1.4 to 4.1.6, we calculate the corresponding column entropies $\mathbb{H}(X)$, $\mathbb{H}(Y)$, and their joint entropy $\mathbb{H}(X, Y)$, respectively.

$$
\begin{aligned}
\mathbb{H}(X) & =-\sum_{i=1}^{20} \hat{p}\left(x_{i}\right) \log \hat{p}\left(x_{i}\right) \\
\mathbb{H}(Y) & =-\sum_{j=1}^{20} \hat{p}\left(y_{j}\right) \log \hat{p}\left(y_{j}\right) \\
\mathbb{H}(X, Y) & =-\sum_{i=1}^{20} \sum_{j=1}^{20} \hat{p}\left(x_{i}, y_{j}\right) \log \hat{p}\left(x_{i}, y_{j}\right)
\end{aligned}
$$

Then, we calculate the

$$
\mathbb{M I}(X ; Y)=-\sum_{i=1}^{20} \hat{p}\left(x_{i}\right) \log \hat{p}\left(x_{i}\right)-\sum_{j=1}^{20} \hat{p}\left(y_{j}\right) \log \hat{p}\left(y_{j}\right)+\sum_{i=1}^{20} \sum_{j=1}^{20} \hat{p}\left(x_{i}, y_{j}\right) \log \hat{p}\left(x_{i}, y_{j}\right),
$$

which can be rewritten as:

$$
\mathbb{M I}(X ; Y)=\sum_{i=1}^{20} \sum_{j=1}^{20} \hat{p}\left(x_{i}, y_{j}\right) \log \frac{\hat{p}\left(x_{i}, y_{j}\right)}{\hat{p}\left(x_{i}\right) \hat{p}\left(y_{j}\right)} .
$$

Finally, we determine the correlation between corresponding columns with $\mathbb{U}$-metric as

$$
\mathbb{U}(X, Y)=2 \cdot \frac{\mathbb{H}(X)+\mathbb{H}(Y)-\mathbb{H}(X, Y)}{\mathbb{H}(X)+\mathbb{H}(Y)}=2 \cdot \frac{\mathbb{M I}(X ; Y)}{\mathbb{H}(X)+\mathbb{H}(Y)}
$$

The higher the $\mathbb{U}(X, Y)$ value between columns $k$ and $l$, the stronger the pair-wise cooccurrence of amino acids at these columns. 


\section{The alphabet size effect on the mutual information}

The mutual information (MII) is a useful method in bioinformatics in order to measure the correlation between two columns in MSAs. However, Martin et al. [8] have argued and showed in detail that the normalizing $\mathbb{M I}$ values with the entropy makes them more suitable than raw MI values for the correlated/compensatory mutation analysis. This is due to possible differences in the alphabet size of a column.

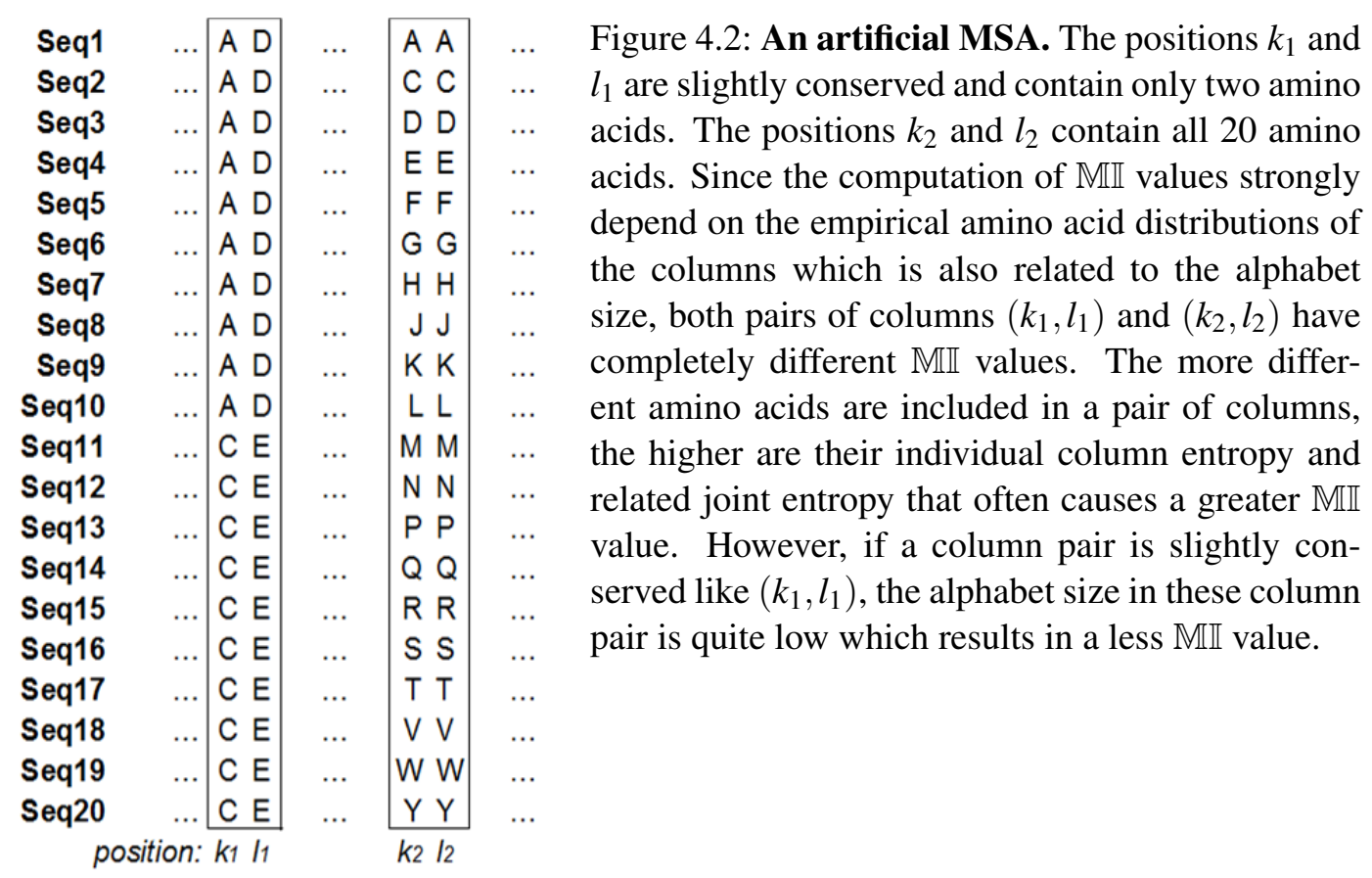

Example: Let $M$ be the MSA under study and let $\left(k_{1}, l_{1}\right)$ and $\left(k_{2}, l_{2}\right)$ be two different column pairs in $M$, where the observed alphabet size of the first column pair is $\mathfrak{X}_{\left(k_{1}, l_{1}\right)}=2$ and the observed alphabet size of the second column pair $\mathfrak{X}_{\left(k_{2}, l_{2}\right)}=20$ (see Figure 4.2). Further, suppose that the associated empirical amino acid distributions, $\hat{p}\left(x_{k_{i}}\right), \hat{p}\left(y_{l_{i}}\right)$ and $\hat{p}\left(x_{k_{i}}, y_{l_{i}}\right), i=1,2$ are uniformly distributed in both pairs of columns which results in

$$
\begin{aligned}
& \mathbb{H}\left(X_{k_{1}}\right)=\mathbb{H}\left(Y_{l_{1}}\right)=\mathbb{H}\left(X_{k_{1}}, Y_{l_{1}}\right), \\
& \mathbb{H}\left(X_{k_{2}}\right)=\mathbb{H}\left(Y_{l_{2}}\right)=\mathbb{H}\left(X_{k_{2}}, Y_{l_{2}}\right) .
\end{aligned}
$$


Now, we calculate the $\mathbb{M I}\left(X_{k_{i}} ; Y_{l_{i}}\right)$ of both pairs of columns as

$$
\begin{aligned}
\mathbb{M I}\left(X_{k_{1}} ; Y_{l_{1}}\right) & =-\sum_{1}^{2} \frac{1}{2} \log \frac{1}{2}=-\log \frac{1}{2}=\log 2=\log \left|\mathfrak{X}_{\left(k_{1}, l_{1}\right)}\right| \\
\mathbb{U}\left(X_{k_{1}} ; Y_{l_{1}}\right) & =2 \cdot \frac{\mathbb{M I}\left(X_{k_{1}} ; Y_{l_{1}}\right)}{\mathbb{H}\left(X_{k_{1}}\right)+\mathbb{H}\left(Y_{l_{1}}\right)}=1 \\
\mathbb{M I}\left(X_{k_{2}} ; Y_{l_{2}}\right) & =-\sum_{1}^{20} \frac{1}{20} \log \frac{1}{20}=-\log \frac{1}{20}=\log 20=\log \left|\mathfrak{X}_{\left(k_{2}, l_{2}\right)}\right| \\
\mathbb{U}\left(X_{k_{2}} ; Y_{l_{2}}\right) & =2 \cdot \frac{\mathbb{M I}\left(X_{k_{2}} ; Y_{l_{2}}\right)}{\mathbb{H}\left(X_{k_{2}}\right)+\mathbb{H}\left(Y_{l_{2}}\right)}=1
\end{aligned}
$$

The Equations 4.1.14 and 4.1.16 clearly show the alphabet size effect on the MII. Although, both column pairs $\left(k_{1}, l_{1}\right)$ and $\left(k_{2}, l_{2}\right)$ are perfectly correlated and have the same $\mathbb{U}$ value, their raw MII-values reflect these correlations completely different because of the alphabet size. Consequently, we can see that the higher is the alphabet size, the greater is the corresponding mutual information. Thus, a normalization method was required in order to reduce the effect of the alphabet size on the $\mathrm{MI}$ values which explains the reason of using the $\mathbb{U}$-metric in this thesis.

\subsubsection{Filtering of an MSA}

We filter MSAs before their analysis with an approach similar to [22].

First, we delete highly similar and dissimilar sequences in a given MSA to ensure that the sequence identity between any two sequences is at least $20 \%$ and no more than $90 \%$.

Second, we remove strictly conserved residue columns (see column $m$ in the Figure 4.1). We say a column is strictly conserved if the percentage of identical residues is greater than $95 \%$. The reason for this is two-fold. First, these strictly conserved columns occur because of the common ancestor of all living organisms thus a compensatory mutation at that positions is often not allowed through the evolution (see Section 2.3. Second, fully conserved columns have an entropy of zero which results in a division through zero (see Section 3.1).

Third, we eliminate the columns which contain more than $25 \%$ gaps. As mentioned before, we do not consider the gaps '-' as a real amino acid in a protein as well as in an MSA. Therefore, rows with a gap at position $k$ or $l$ were excluded from the computation of $\mathbb{U}$ metric. If we incorporate them in the calculation, the gaps can provide wrong information on the correlation between columns. As a result of this, the columns with a higher percentage of gaps could be detected by the $\mathbb{U}$-metric as highly correlated although only little information is available in those columns.

Finally, we only consider MSAs that have at least 125 rows remaining after the filtering. 


\subsection{Determining an MSA-specific lower bound for the significance of $\mathbb{U}$-values}

Filtering an MSA ensures that there is always a correlation between each column pairs in this MSA based on the definition of $\mathbb{U}$-metric. However, a challenging problem in bioinformatics is the separation of significant $\mathbb{U}$-values between two or more column pairs from the background noise and unrelated column pairs. Thus, we have developed an MSA-specific statistical model based on multiple testing procedures that quantifies the error made in terms of the false discovery rate described in [27,28]. Below, we will explain our statistical model step by step. The result is an MSA-dependent threshold $\tau$ above which $\mathbb{U}$-values are defined as significant.

\subsubsection{Step 1: Calculation of $p$-values}

Let $M$ be the MSA for the protein under study. We slightly extend the standard approach of multiple testing procedures introduced in [27, 75, -77] with the following assumptions in mind. If regarded as random variables, $M$ 's $\mathbb{U}(k, l)$-values follow three different distributions as demonstrated in Figure 4.3 , i) a null distribution, $F_{0}$ representing background signals; ii) a distribution for those column pairs which are completely unrelated, $G_{1}$; iii) a distribution representing the correlation signals we are interested in, $G_{2}$.

We assume $F_{0}$ to be a $\beta$-distribution and $M$ 's $\mathbb{U}(k, l)$-values $U_{1}, U_{2}, \ldots, U_{\mu}$ be an independent and identically distributed (i.i.d.) sample, although there are some weak dependencies between them. With respect to the $F_{0}$, we first determine the $p$-values of each $\mathbb{U}$ values.

\section{p-values of $\mathbb{U}$-values}

The $p$-values of $\mathbb{U}$ depend on whether $\mathbb{U}$-values are $F_{0}, G_{1}$ or $G_{2}$-distributed. According to Figure 4.4 and the definition of $\mathbb{U}$-metric, we can say that while the $G_{1}$-distributed unrelated pair signals have a low $\mathbb{U}$-value, the $G_{2}$ distributed correlation signals of column pairs take relatively high $\mathbb{U}$-values, whereas the $F_{0}$ distributed background signals have moderate $\mathbb{U}$ values.

Let $M$ be an arbitrarily chosen but fixed MSA and $U_{1}, U_{2}, \ldots, U_{\mu}$ be $M$ 's $\mathbb{U}$-values. We calculate the $p$-value for each $\mathbb{U}$-value as

$$
X_{l}=1-F_{0}\left(U_{l}\right)=P\left\{\text { random } F_{0} \text {-distributed value } \geq U_{l}\right\},
$$

where $X_{l}$ is the $p$-value of $U_{l}$ with respect to $F_{0}$. If $U_{l}$ is $F_{0}$-distributed, then $X_{l}$ is uniform over [0,1] (Figure 4.3). If however, $U_{l}$ is $G_{1}$ distributed, then the corresponding $X_{l}$ is relatively high and tends to 1 (Figure 4.3). In contrast, if $U_{l}$ is $G_{2}$ distributed, then $X_{l}$ becomes a low score and tends to 0 (Figure 4.3 . 


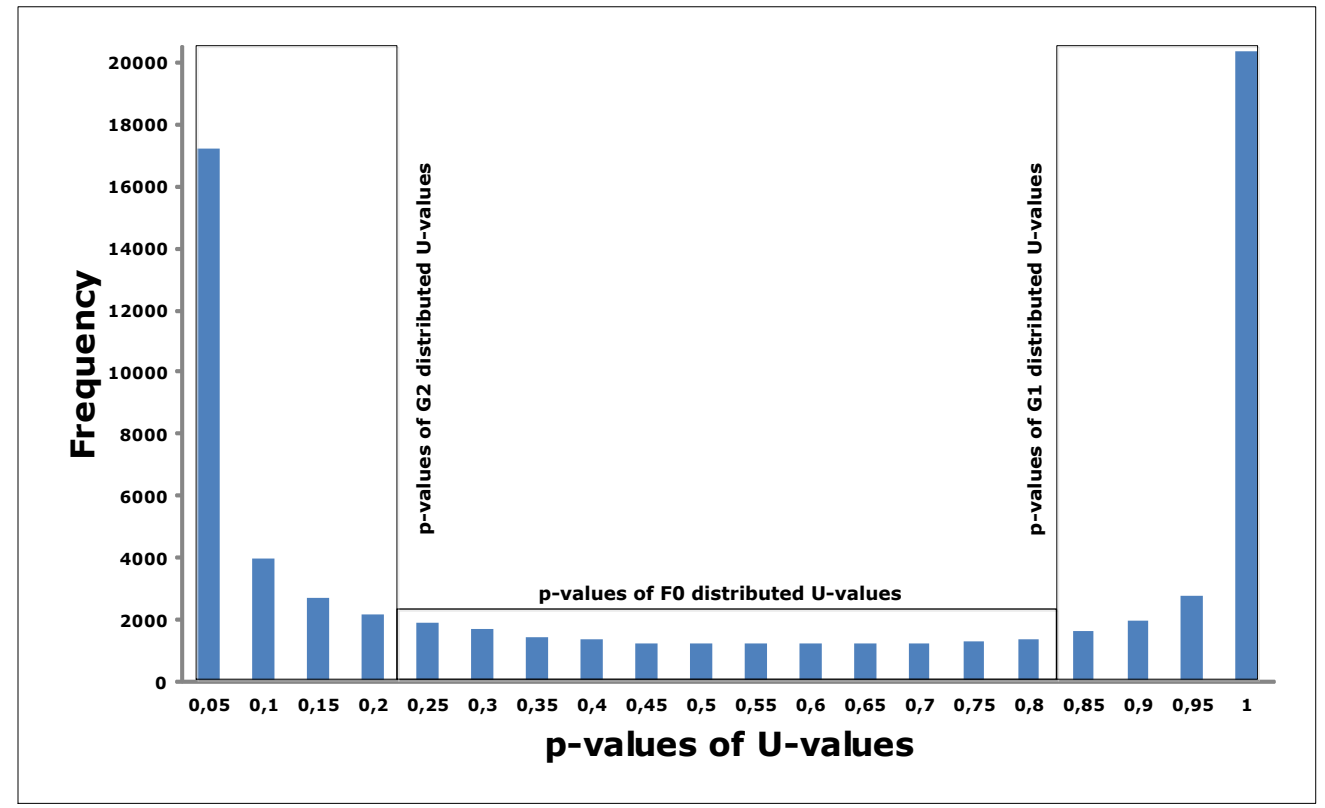

Figure 4.3: p-value distribution of U-values for human GCK protein (PDB Entry 1V4S). The p-values close to zero represent the significant pairs by means of which we assess the individual residue position. In contrast, $\mathrm{p}$-values of unrelated pairs tend to one. As one can see, the p-values of $F_{0}$ distributed $\mathbb{U}$-values are approximately uniform.

Equation 4.2.1 shows that the $p$-values of the $\mathbb{U}$-values can be calculated based on either the cumulative distribution function of the $\beta$-distribution (see Equation 3.3.5) or by drawing a random sample from the $\beta$-distribution with parameters $\alpha, \beta>0$. However, in both cases we first need to know the $\alpha$ and $\beta$. According to equations 3.3.6 and 3.3.7, we can estimate $\alpha$ and $\beta$ from the expected value and the variance of $M$ 's $\mathbb{U}$-values. The expected value is estimated by the sample mean of all $\mathbb{U}$-values of $M$ according to the Equation 3.3.8. However, we do not estimate the variance directly using the Equation 3.3.9, instead we use a more sophisticated approach. If we were to use the Equation 3.3.9 directly, we would get relatively high sample variance because of the $G_{1}$ and $G_{2}$-distributed $\mathbb{U}$-values. Then, the $\beta$ - distribution would not represent the $F_{0}$ - distribution. Hence, the corresponding $p$-values do not follow the uniform distribution over $[0,1]$ (see Figure 4.5).

follow the real scores (see figure 4.5).

In order to eliminate the effect of $G_{1}$ and $G_{2}$-distributed $\mathbb{U}$-values on the sample variance we take pattern from [78]. Having drawn an i.i.d. sample $\left(C_{1}, C_{1}^{\prime}\right),\left(C_{2}, C_{2}^{\prime}\right), \ldots,\left(C_{v}, C_{v}^{\prime}\right)$ of random column pairs of a sufficient size whose $\mathbb{U}$-values fall in a preassigned subinterval 


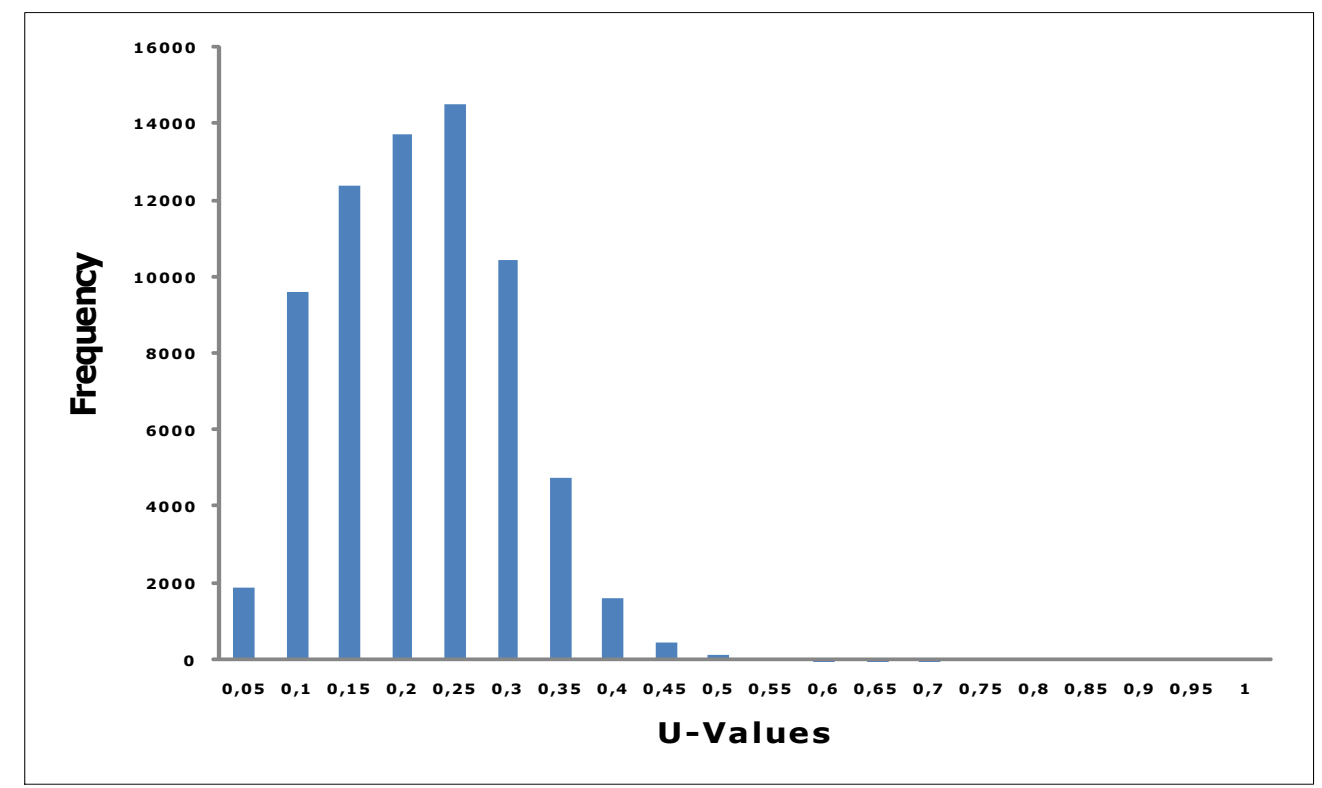

Figure 4.4: U-value distribution for human GCK protein (PDB Entry 1V4S). The higher the $\mathbb{U}$-value between pairs of columns, the stronger the correlation of amino acids at these columns.

of $[0,1]$, we calculate $D_{1}, D_{2}, \ldots, D_{v}$ by randomly shuffling the sequence $C_{l}^{\prime}, \imath=1,2, \ldots, v$. The artificial variance is then estimated according to Equation 3.3.9 as the sample variance of $\left(C_{1}, D_{1}\right),\left(C_{2}, D_{2}\right), \ldots,\left(C_{v}, D_{v}\right)$. Afterwards, we use the sample mean and the artificial variance to estimate the parameters of the $\beta$-distribution.

In order to draw random numbers from the $\beta$-distribution, we apply the $B N$-algorithm in [79], which is an acceptance-rejection technique using uniform and normal distributions to generate $\beta$-distributed random numbers (see Algorithm 1 ).

\subsubsection{Step 2: Setting an MSA-dependent threshold $\tau$}

After the computation of $p$-values of $M$ 's $\mathbb{U}$-values, we need a threshold $\tau$ to separate significant $p$-values of correlation signals from the background and unrelated pair signals. While determining such a significant threshold $\tau$, a certain amount of non-significant $\mathbb{U}$-values is accepted to be significant. The ratio of these falsely accepted values is defined as False Discovery Rate (FDR). In order to determine an MSA-dependent threshold $\tau$ above which $\mathbb{U}$ - 


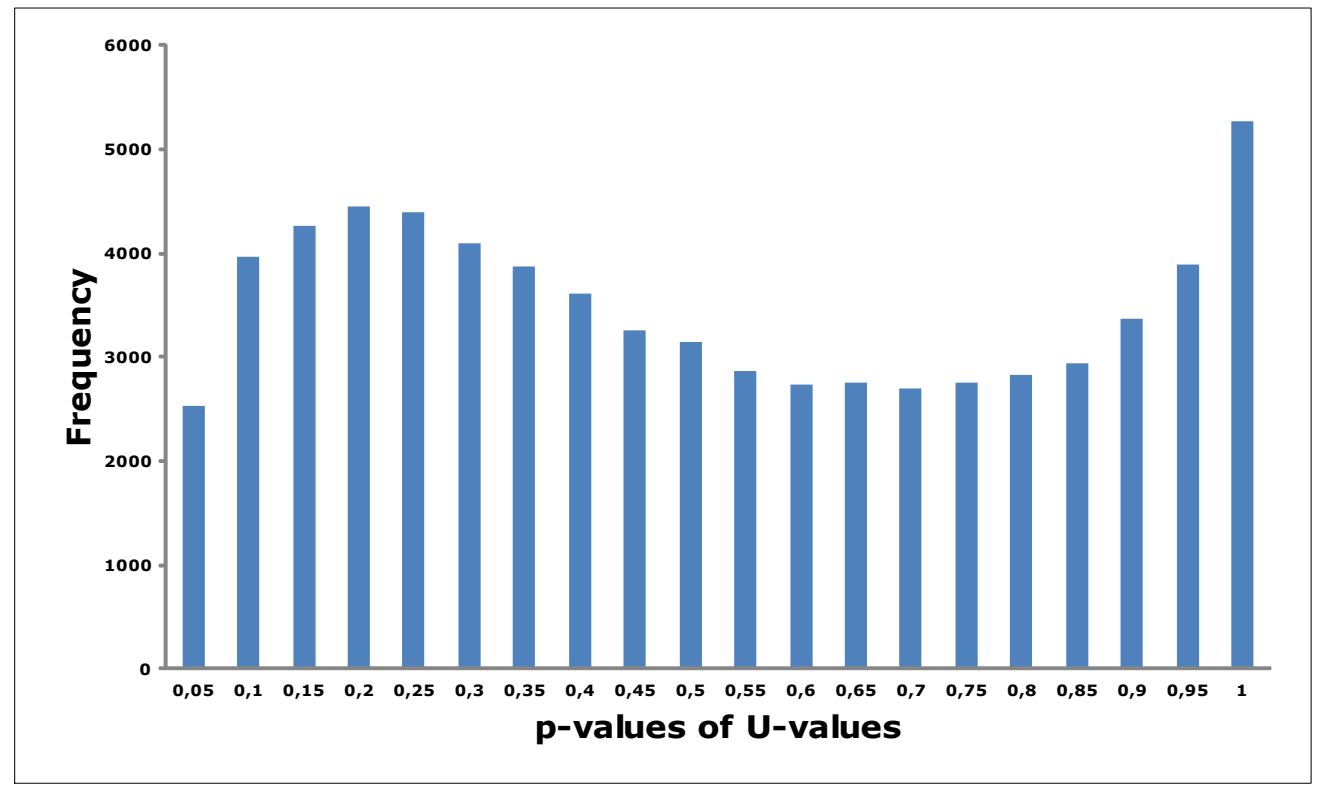

Figure 4.5: p-value distribution of $\mathbb{U}$-values according to the sample mean and variance for human GCK protein (PDB Entry 1V4S). The $p$-values are estimated based on the sample mean and variance which is calculated using all $\mathbb{U}$-values of $M$. As one can see, if we use directly the sample variance for the estimation of shape parameters $\alpha$ and $\beta$ of the $\beta$-distribution, the $p$-values of $\mathbb{U}$-values do not follow the uniform distribution over $[0,1]$.

values are defined as significant, we generalize for a preassigned FDR the Storey-Tibshirani procedure devised for multiple testing problems as follows [27, 28].

First, we estimate the fraction $\gamma$ of the $F_{0}$-distributed $U_{l}$ 's as

$$
\hat{\gamma}=\frac{\text { number of } p \text {-values in }\left[\lambda_{1}, \lambda_{2}\right]}{n\left(\lambda_{2}-\lambda_{1}\right)}
$$

where $n$ is the total number of $M$ 's $\mathbb{U}$-values and $\lambda_{1}, \lambda_{2}$ are the tuning parameters. $\lambda_{1}$ and $\lambda_{2}$ are chosen such that the fraction of not uniformly distributed $p$-values that fall into $\left[\lambda_{1}, \lambda_{2}\right]$ is negligible. The selection of the tuning parameters requires an automated approach during the analysis of $M$. Algorithm 2 presents our approach for determining $\lambda_{1}$ and $\lambda_{2}$.

Second, knowing the fraction $\hat{\gamma}$ of the $F_{0}$-distributed $\mathbb{U}$-values, we say a $\mathbb{U}$-value of sites $(i, j)$ is significant if and only if the $p$-value $\left(1-F_{0}(\mathbb{U}(i, j))\right)$ is less than or equal to $\tau$, for 


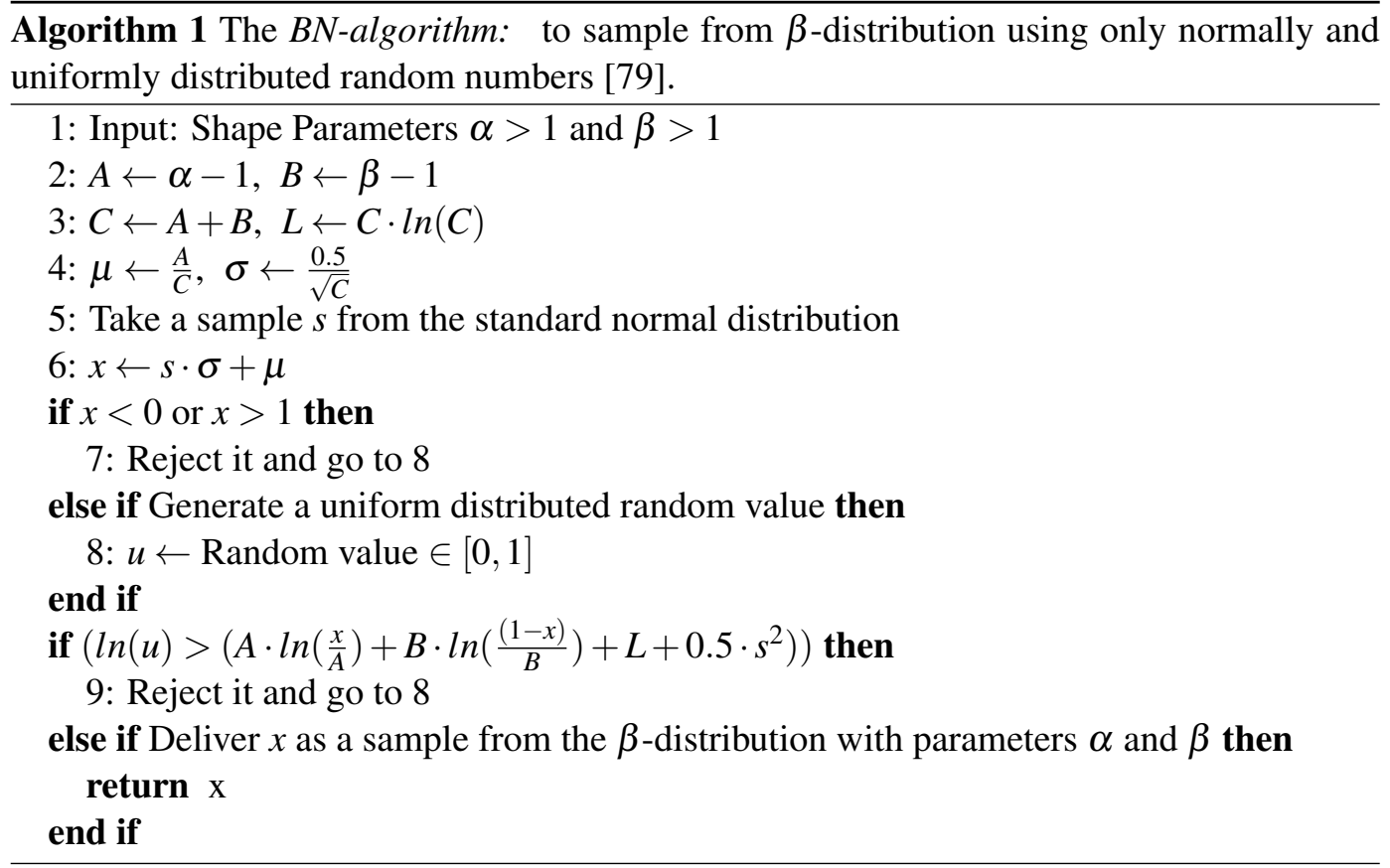

a threshold $\tau \leq \lambda_{1}$ that ensures the input FDR, which is estimated by

$$
\widehat{\operatorname{FDR}}(\tau)=\frac{\hat{\gamma} n \tau}{\text { number of } p \text {-values } \leq \tau}
$$

Finally, setting $\tau=0$ initially, we increase the $\tau$ step by step and solve the Equation 4.2.3 iteratively, as long as the result of this computation is less than the preassigned $\widehat{\text { FDR }}$. After that, the last $\tau$ upon what the $\widehat{\text { FDR }}$ exceeds its limit is defined as the significance threshold.

\section{Assessment of individual residue sites}

A significant $\mathbb{U}$-value simply reflects the correlation between residue sites $i$ and $j$ in an MSA $M$ but does not provide enough information about the site $i$ or $j$, alone. Because of this, Merkl and Zwick have suggested a method based on concepts of network analysis to characterize individual residue sites [22]. They argued that the consideration of $\mathbb{U}$-values is only a promising method if there is a strong correlation signal between residue sites $i$ and $j$. However, if all $\mathbb{U}$-values are low in an MSA, a single $\mathbb{U}(i, j)$-value can be easily misclassified due to definition of $\mathbb{U}$ metric. To overcome this problem and to evaluate individual residue positions $i$ and $j$, we use the connectivity degree technique introduced in [22] and further developed in [4]. 


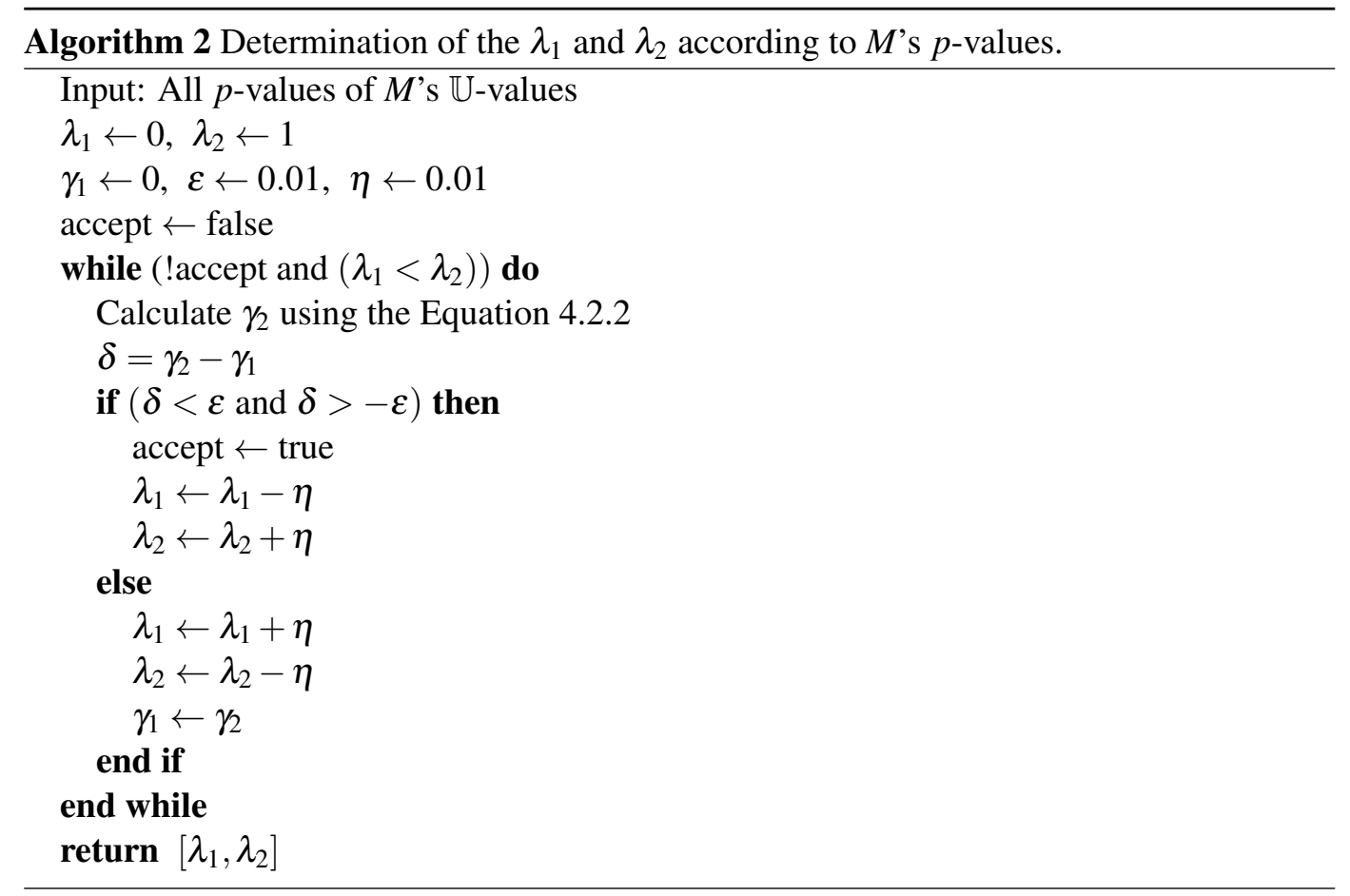

Definition 4.1 (Connectivity degree) The connectivity degree of a site $i$ with respect to the metric $\mathbb{U}$ and the MSA $M$ is defined as number of sites $j$ such that the $\mathbb{U}(i, j)$-value of residue sites $i$ and $j$ is significant for $M$ (see Figure 4.6).

Finally, the site $i$ is defined to be ( $\mathbb{U}, M$ )-significant, if $i$ 's connectivity degree with respect to $\mathbb{U}$ and $M$ is greater than or equal to the $n$-th percentile, where we set $n=90$ in this thesis.

\subsection{Enhancing prediction by the $\mathbb{U}_{D(\alpha)}$-metric that models dissimilar compensatory mutations}

In this section, we will present our new entropy based metric, called $\mathbb{U}_{D(\alpha)}$-metric. The $\mathbb{U}$ -metric introduced in Section 4.1 is defined as a normalized mutual information that uses only the observed frequency of amino acids in the MSA columns. Thus, it does not consider any physical or biochemical properties of amino acids which are likely to be crucial for the detection of functional or structural important positions. Therefore, we have developed our novel $\mathbb{U}_{D(\alpha)}$-metric which differ from the $\mathbb{U}$-metric by incorporating the significant $B L O$ SUM62 dissimilar amino acid signals in the prediction of functional or structural important residue sites. Basically, the calculation of the $\mathbb{U}_{D(\alpha)}$-metric is based on transforming the empirical pair distributions of column pairs with a doubly stochastic pair substitution ma- 


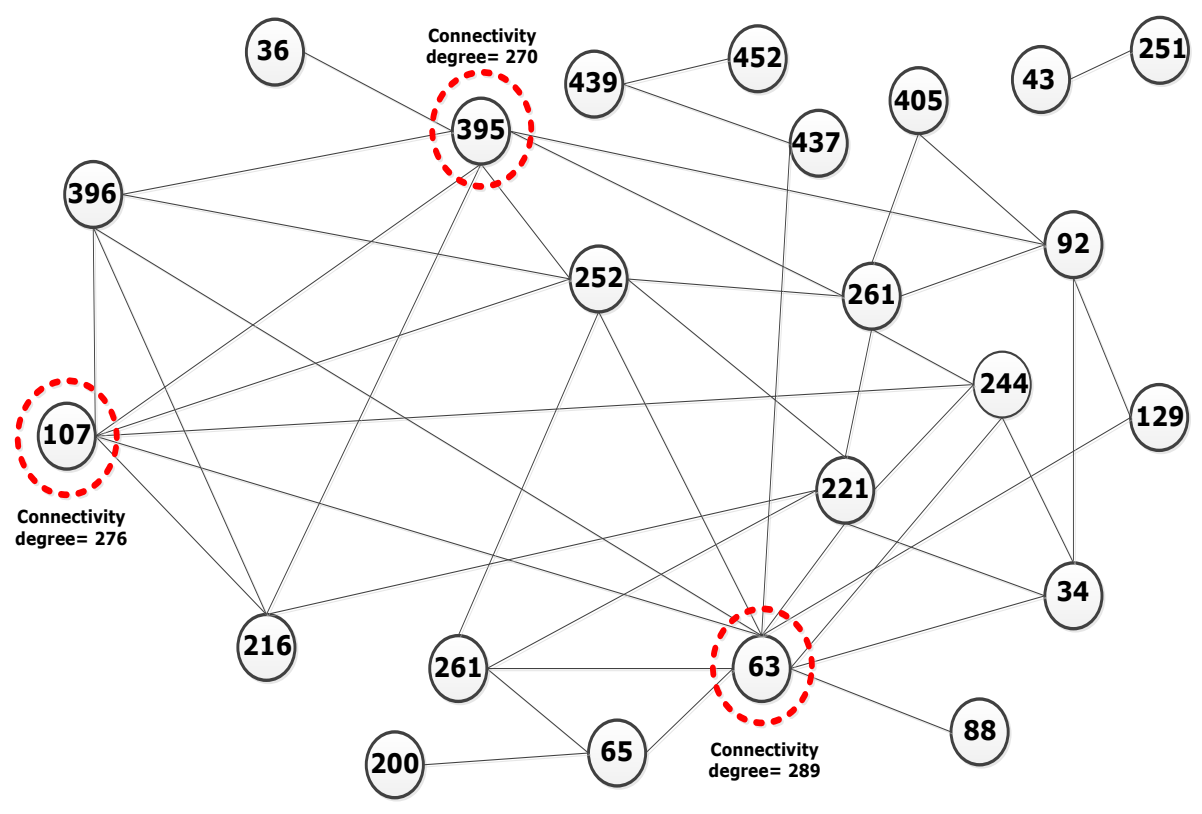

Figure 4.6: A small part of connectivity degree network of human GCK protein (PDBEntery 1V4S): The nodes represent individual residue sites and each vertex indicate a significant correlation (UU-value) between residue sites $k$ and $l$. The vertex count of a node indicates its connectivity degree. The red circles shows residue sites which have three highest connectivity degree in whole network and thus defined as $(\mathbb{U}, M)$-significant.

trix. The doubly stochastic matrix reflects the BLOSUM62 dissimilar amino acid signals which are observed according to the significant column pairs of training MSAs.

\subsubsection{Training data set}

In order to build our mathematical model we used approximately 1700 protein structures which were randomly chosen from a redundancy free data set with more than 35000 protein structures. The redundancy free data set was prepared by Rainer Merkl's group University of Regensburg. In order to construct the data set, the PISCES server [80] is firstly used with a sequence-similarity cut-off of $25 \%$ for the elimination of redundant proteins. Afterwards, we took the corresponding protein structures from the PDB database and the related MSAs were gathered from the HSSP database for our training. Then, we filtered the MSAs in the same way as described in the Section 4.1.1. 
- highly similar and dissimilar sequences in the MSAs are removed to ensure that the sequence identity $\left(s_{i j}\right)$ between any two sequences is $20 \% \leq s_{i j} \leq 90 \%$,

- strictly conserved columns in MSAs which contain same residue types more than 95\% are removed,

- the columns which contain more than $25 \%$ gaps are removed,

- finally, all MSAs with less than 125 sequences are discarded.

More than 17000 MSAs survived the last filtering step. We used approximately 1700 MSAs as training data which we randomly chose from this set. The pdb entries of the corresponding protein structures are listed in Table 9.1

\subsubsection{Preparing substitution matrices}

For our approach, we need to prepare a significant substitution matrix and a random substitution matrix.

Let $M$ be an arbitrarily chosen but fixed MSA with $m$ sequences and let $k$ and $l$ be two columns in $M$. To compute the pair-to-pair substitution scores of amino acid pairs in these columns, we utilize standard concept of computational biology suggested in [81, 82]. For the 20 amino acids, there are 400 different amino acid pairs and each of them can occur in this column pair $(k, l)$. Since we count pair-to-pair substitution of amino acid pairs, there exist 160000 different possible substitution between amino acid pairs for which we use a $400 \times 400$ symmetric matrix $C$. Having that, we calculate pair-to-pair substitution score between pairs of amino acids $\left[a_{k i}, a_{l i}\right]$ and $\left[a_{k j}, a_{l j}\right]$ occurring in columns $k$ and $l$ based on counting the following four individual substitutions:

$$
\begin{aligned}
& \left(\left[a_{k i}, a_{l i}\right],\left[a_{k j}, a_{l j}\right]\right), \\
& \left(\left[a_{l i}, a_{k i}\right],\left[a_{l j}, a_{k j}\right]\right), \\
& \left(\left[a_{k j}, a_{l j}\right],\left[a_{k i}, a_{l i}\right]\right), \\
& \left(\left[a_{l j}, a_{k j}\right],\left[a_{l i}, a_{k i}\right]\right),
\end{aligned}
$$

where $i$ and $j$ indicate the corresponding rows in $M$. We count the substitutions between pairs of amino acids symmetrically since the correct order of the sequences in MSAs is unknown. Finally, each of these substitution scores are summed up in the matrix $C$. In Figure 4.7 we give an example in order to show how we count the pair-to-pair substitution in a column pair.

\subsubsection{Creating the Doubly Stochastic Matrix to model dissimilar compensatory mutations}

A pair $\left(\left(a_{i}, a_{j}\right),\left(a_{k}, a_{l}\right)\right)$ of amino acid pairs is defined to be a formal dissimilar compensatory mutation, if the BLOSUM62 score of both $\left(a_{i}, a_{k}\right)$ and $\left(a_{j}, a_{l}\right)$ is negative. 


Human/1-448
Monkey/14-461
Chimpanzee/14-461
Mouse/14-461
Rat/14-444
Horse/14-461
Cow/3-447
Chicken/14-461
Frog/14-461
Zebrafish/14-461
SeaUrchin/14-444



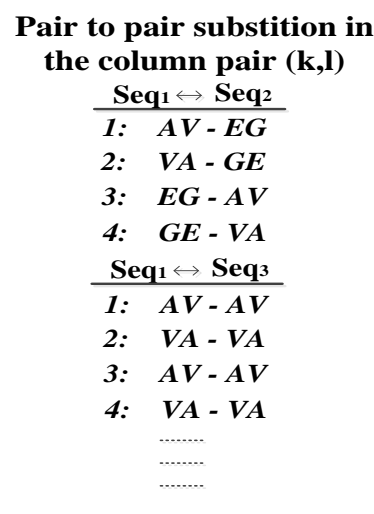

Figure 4.7: Counting pair to pair substitution: In the column pair $(k, l)$ occurs different pair to pair substitution such as $([A, V] \leftrightarrow[E, G]),([A, V] \leftrightarrow[A, V])$ and $([E, G] \leftrightarrow[E, G])$ or vice versa. Since the correct order of the sequences in MSAs is unknown, we count the substitutions symmetrically and store the occurrence values of substitutions in a matrix $C$.

We use the training data set to estimate a $400 \times 400$ doubly stochastic matrix $D_{\text {CompMut }}$. This matrix is our mathematical model of how dissimilar compensatory mutations have affected genomic sequences in the course of evolution. Its construction consists of following five steps.

\section{Step 1:}

We calculate a signal and a null set of column pairs. The signal set consists of all (U, $M)$ significant column pairs, where $M$ ranges over all training MSAs. Significant column pairs of each training MSA $M$ are determined individually using the MSA-specific statistical model (see Section 4.2). The null set consists of sufficiently many non-significant column pairs randomly chosen from every training MSA. For both the signal set and the null set we compute a symmetric $400 \times 400$ integer-valued matrix of frequencies of pair substitutions $C_{\text {alt }}$ and $C_{\text {null }}$ as explained in Section 4.3.2.

\section{Step 2:}

We compare both $C_{\text {alt }}$ and $C_{\text {null }}$ matrices in order to find out if a substitution score of signal pairs is smaller than pure chance. 
Hence, we define a new matrix $C_{\text {sig }}$ by

$$
C_{\text {sig }}\left(\left(a_{i}, a_{j}\right),\left(a_{k}, a_{l}\right)\right)= \begin{cases}C_{\mathrm{alt}}\left(\left(a_{i}, a_{j}\right),\left(a_{k}, a_{l}\right)\right) & \text { if } \varphi\left(\left(a_{i}, a_{j}\right),\left(a_{k}, a_{l}\right)\right)=1 ; \\ 0 & \text { otherwise }\end{cases}
$$

where $\varphi\left(\left(a_{i}, a_{j}\right),\left(a_{k}, a_{l}\right)\right)=1$ if and only if $\left(a_{i}, a_{j}\right)=\left(a_{k}, a_{l}\right)$ or

$$
\frac{C_{\mathrm{alt}}\left(\left(a_{i}, a_{j}\right),\left(a_{k}, a_{l}\right)\right)}{\sum_{i^{\prime}, j^{\prime}, k^{\prime}, l^{\prime}} C_{\mathrm{alt}}\left(\left(a_{i^{\prime}}, a_{j^{\prime}}\right),\left(a_{k^{\prime}}, a_{l^{\prime}}\right)\right)}>\frac{C_{\mathrm{null}}\left(\left(a_{i}, a_{j}\right),\left(a_{k}, a_{l}\right)\right)}{\sum_{i^{\prime}, j^{\prime}, k^{\prime}, l^{\prime}} C_{\mathrm{null}}\left(\left(a_{i^{\prime}}, a_{j^{\prime}}\right),\left(a_{k^{\prime}}, a_{l^{\prime}}\right)\right)} .
$$

\section{Step 3:}

We set all entries of the matrix $C_{\text {sig }}$ outside the main diagonal that do not represent a formal dissimilar compensatory mutation to zero.

$$
C_{\text {CompMut }}\left(\left(a_{i}, a_{j}\right),\left(a_{k}, a_{l}\right)\right)=\left\{\begin{array}{l}
0, \text { if BLOSUM62 score of }\left(a_{i}, a_{k}\right) \text { or }\left(a_{j}, a_{l}\right) \geq 0 \\
C_{\mathrm{sig}}\left(\left(a_{i}, a_{j}\right),\left(a_{k}, a_{l}\right)\right), \text { otherwise }
\end{array}\right.
$$

This results in the compensatory mutation matrix $C_{\mathrm{CompMut}}$. By normalizing $C_{\mathrm{CompMut}}$, we obtain a symmetric matrix $P_{\text {CompMut }}$. For $a_{i}, a_{j}, a_{k}, a_{l}$ ranging over all amino acids, $P_{\text {CompMut }}\left(\left(a_{i}, a_{j}\right),\left(a_{k}, a_{l}\right)\right)$ represents an empirical probability distribution on pairs of amino acid pairs.

\section{Step 4:}

Afterwards, we calculate the symmetric $400 \times 400$-matrix

$$
S_{\text {CompMut }}=\left(\log \frac{P_{\text {CompMut }}\left(\left(a_{i}, a_{j}\right),\left(a_{k}, a_{l}\right)\right)}{P_{\text {CompMut }}^{\mathrm{b}}\left(a_{i}, a_{j}\right) P_{\text {CompMut }}^{\mathrm{b}}\left(a_{k}, a_{l}\right)}\right)_{\left(a_{i}, a_{j}\right),\left(a_{k}, a_{l}\right)},
$$

where $P_{\text {CompMut }}^{\mathrm{b}}\left(a_{i}, a_{j}\right)$ is the marginal distribution of $P_{\mathrm{CompMut}}$.

\section{Step 5:}

We set all negative entries of $S_{\text {CompMut }}$ to zero. Then we compute the doubly stochastic matrix $D_{\text {CompMut }}$ by means of the canonical iterated row-column normalization procedure [83]. 


\subsubsection{Application of doubly stochastic matrix $D_{\text {CompMut }}$ for the computation of the new $\mathbb{U}_{D(\alpha)}$-metric}

In the previous Section 4.3 .3 , the matrix $D_{\text {CompMut }}$ has been estimated to model significant and BLOSUM62 dissimilar compensatory mutation signals. Based on the matrix $D_{\mathrm{CompMut}}$, we incorporate compensatory mutation signals in the computation of the $\mathbb{U}$-metric that results in our novel $\mathbb{U}_{D(\alpha)}$-metric. Consequently, for every column pair $(i, j)$ of the input MSA $M$, we linearly transform the associated empirical pair distribution with the doubly stochastic matrix as

$$
D(\alpha)=(1-\alpha) \mathbf{1}+\alpha D_{\mathrm{CompMu}},
$$

where 1 is the $400 \times 400$ unit matrix and $\alpha \in(0,1]$ is a preassigned real number. $\mathbb{U}_{D(\alpha)}(i, j)$ is then defined to be the $\mathbb{U}$-value (see Equation 4.1.1) of this transform.

In the following example, we demonstrate how the empirical pair distribution of a column pair in an MSA is transformed with the doubly stochastic matrix to calculate corresponding $\mathbb{U}_{D(\alpha)^{- \text {value. }}}$

Example: Let $M$ be the input MSA and $(k, l)$ be a column pair in $M$ with empirical joint distributions of amino acid pairs $\hat{p}\left(x_{i}, y_{i}\right)=\hat{p}\left\{X=x_{i}, Y=y_{i}\right\}, x, y \in \mathfrak{X}$, and $i=1,2, \ldots, 20$. We write these pair probabilities $\hat{p}\left(x_{i}, y_{i}\right)$ in a $\hat{P}_{20 \times 20}$ matrix.

\begin{tabular}{|c|ccc|c|}
\hline & $y_{1}$ & $\cdots$ & $y_{20}$ & $\sum_{j} \hat{p}\left(x_{i}, y_{j}\right)$ \\
\hline$x_{1}$ & $\hat{p}\left(x_{1}, y_{1}\right)$ & $\cdots$ & $\hat{p}\left(x_{1}, y_{20}\right)$ & $\hat{p}_{x}\left(x_{1}\right)$ \\
$\vdots$ & $\vdots$ & $\ddots$ & $\vdots$ & $\vdots$ \\
$x_{20}$ & $\hat{p}\left(x_{20}, y_{1}\right)$ & $\ldots$ & $\hat{p}\left(x_{20}, y_{20}\right)$ & $\hat{p}_{x}\left(x_{20}\right)$ \\
\hline$\sum_{i} \hat{p}\left(x_{i}, y_{j}\right)$ & $\hat{p}_{y}\left(y_{1}\right)$ & $\cdots$ & $\hat{p}_{y}\left(y_{20}\right)$ & 1 \\
\hline
\end{tabular}

Table 4.1: $\hat{P}_{20 \times 20}$ : Observed probabilities of amino acid pairs in column pair $(k, l)$, where $x_{i}, y_{j} \in 20$ amino $\operatorname{acids}_{(A, C, D, E, F, G, H, I, K, L, M, N, P, Q, R, S, T, V, W, Y)}$.

Then, the $\hat{P}_{20 \times 20}$ matrix is converted in a vector $\vec{v}$ of length 400 which is necessary to linearly transform the associated pair distribution $\hat{p}(x, y)$ with doubly stochastic matrix $D_{\text {CompMut }}$.

$$
\vec{v}_{400}^{*}=\vec{v} \times D_{\mathrm{CompMut}}
$$

After that the vector $\vec{v}_{400}^{*}$ is converted again in a $\hat{P}^{*} 20 \times 20$ matrix to determine the novel transformed marginal column distributions $\hat{p}^{*}(x), \hat{p}^{*}(y)$ and pair distribution $\hat{p}^{*}(x, y)$. 


\begin{tabular}{|c|ccc|c|}
\hline & $y_{1}$ & $\cdots$ & $y_{20}$ & $\sum_{j} \hat{p}^{*}\left(x_{i}, y_{j}\right)$ \\
\hline$x_{1}$ & $\hat{p}^{*}\left(x_{1}, y_{1}\right)$ & $\cdots$ & $\hat{p}^{*}\left(x_{1}, y_{20}\right)$ & $\hat{p}^{*}{ }_{x}\left(x_{1}\right)$ \\
$\vdots$ & $\vdots$ & $\ddots$ & $\vdots$ & $\vdots$ \\
$x_{20}$ & $\hat{p}^{*}\left(x_{20}, y_{1}\right)$ & $\ldots$ & $\hat{p}^{*}\left(x_{20}, y_{20}\right)$ & $\hat{p}^{*}{ }_{x}\left(x_{20}\right)$ \\
\hline$\sum_{i} \hat{p}^{*}\left(x_{i}, y_{j}\right)$ & $\hat{p}^{*}\left(y_{1}\right)$ & $\cdots$ & $\hat{p}^{*}\left(y_{20}\right)$ & 1 \\
\hline
\end{tabular}

Table 4.2: $\hat{P}^{*} 20 \times 20$ : Transformed values of joint amino acid pair distribution

Finally, in order to calculate our new $\mathbb{U}_{D(\alpha)}$-metric, we determine the individual column entropies $\mathbb{H}\left(k^{*}\right), \mathbb{H}\left(l^{*}\right)$ and joint entropy $\mathbb{H}\left(k^{*}, l^{*}\right)$ based on the transformed distributions $\hat{p}^{*}(x), \hat{p}^{*}(y), \hat{p}^{*}(x, y)$, respectively.

$$
\mathbb{U}_{D(\alpha)}(k, l)=2 \cdot \frac{\mathbb{H}\left(k^{*}\right)+\mathbb{H}\left(l^{*}\right)-\mathbb{H}\left(k^{*}, l^{*}\right)}{\mathbb{H}\left(k^{*}\right)+\mathbb{H}\left(l^{*}\right)}
$$

Having canonically carried over the definition of a significant site pair and of the connectivity degree of a site to this case, a site $i$ is called $\left(\mathbb{U}_{D(\alpha)}, M\right)$-significant, if $i$ 's connectivity degree with respect to the metric $\mathbb{U}_{D(\alpha)}$ is greater than or equal to the 90 -th percentile. 


\subsection{Discussion}

To predict sites of structural or functional importance, we combine the known $\mathbb{U}$-metric of normalized mutual information [22] with our novel metric $\mathbb{U}_{D^{(i)}(1)}$ to enhance the influence of dissimilar compensatory mutations when measuring covariation of two sites. We discuss how we devised $\mathbb{U}_{D(1)}$ introduced in the Section 4.3 .

To learn the frequency of compensatory mutations, we took $\mathbb{U}$-significant site pairs as training data. We did that for reasons of computation time regardless of the fact that these data are biased. To deal with this bias, one could carry through the training in an iterative process, with our training being the first iteration. For $i>0$, in the $(i+1)$-th iteration of this modified training, a doubly stochastic matrix $D_{\text {CompMut }}^{(i+1)}$ is calculated based on $\mathbb{U}_{D^{(i)}(1)}$-significant site pairs. This is done until the training data are stable.

According to Birkhoff's Theorem [84], every doubly stochastic matrix is a convex combination of permutation matrices. Moreover, from the Hardy-Littlewood-Pólya majorization theorem [85] follows that transforming the probability mass function by a doubly stochastic matrix increases entropy. Consequently, by linearly transforming the empirical amino acid pair distribution of a site pair by $D(1)$ before calculating the $\mathbb{U}$-value, we penalized those site pairs whose original distribution does not match the frequency pattern of formal dissimilar compensatory mutations in the training data described in the Section 4.3.1.

The challenge was to separate the signal caused by structural and functional constraints from the background and unrelated pair signal. To address this issue, we studied only metrics $\mu$ that satisfy the following condition. The larger the $\mu(k, l)$-value, the larger the probability that the two sites $k$ and $l$ have co-evolved. Our critical assumptions were: i) the $\mu(k, l)$-values follow three different distributions, one for the signal, one for the noise, and one for pairs of completely unrelated sites; ii) there is an MSA-dependent threshold below which the metric $\mu$ does not fall with overwhelming probability, when it is applied to the site pairs of functional or structural importance to which $\mu$ is sensitive; iii) there is an MSAdependent threshold significantly smaller then the one in (ii) such that with overwhelming probability there are no $\mu(k, l)$-values of pairs $(k, l)$ of unrelated sites exceeding it.

In order to near-completely eliminate the noise, we filtered both our training and input data. We calculated the significant pairs such that the preassigned false discovery rate was guaranteed by generalizing the Storey-Tibshirani procedure devised for multiple testing problems [27].

Our method to eliminate noise is orthogonal to the technique developed in [9]. Therein, for every pair of sites the so-called average product correction (APC) is calculated as an explicit noise measure, by which the mutual information is then decreased. Furthermore, it generalizes the way Merkl and Zwick [22] as well as Gao et al. [23] cope with noise. According to our judgment, taking only the top 75 high-scoring pairs or the top 25 pairs into account as has been suggested in [22] and [23], respectively, is too conservative. 
We based our noise separation technique on rather weak distribution assumptions that are standard practice in multiple hypothesis testing, instead of explicitly model the noise in terms of a metric. We applied the connectivity degree technique due to Merkl and Zwick [22] to significant site pairs with respect to our both metrics. 


\section{Applying quantum information theory for the detection of functionally or structurally important sites}

In this chapter, we will develop our second model for the prediction of important sites in proteins by applying quantum information theory. First, we give an introduction how the notions of this theory are mapped onto those from bioinformatics. After that, we will define two new metrics based on quantum Jensen-Shannon divergence in order identify functionally and/or structurally important residue sites in MSAs.

We publish the context of this chapter in [5] (see Appendix B 9.2). The descriptions and notations are based on this publication.

\subsection{Notation mapping from quantum information theory in protein bioinformatics}

The 20 amino acids are associated with the standard basis vectors of a 20-dimensional real vector space $\mathfrak{A}$ of column vectors. It is important to note that, we only deal with real vector spaces for our model.

As has been mentioned in the Section 3.2, in Dirac notation a column vector $\psi$ is represented by a ket $|\psi\rangle$. Thus, the 20 amino acids are associated with the basis $|1\rangle,|2\rangle, \ldots,|20\rangle$ of $\mathfrak{A}$, where $|i\rangle$ is the column vector whose $j$-th entry is equal to 1 if $i=j$, and equal to 0 otherwise.

In quantum mechanics unit-length vectors are the states of the system under study. The larger the scalar product of states, the better they are aligned. Thus, we interpret the scalar product of states as similarity measure. If in turn certain not necessarily orthogonal states represent amino acids or amino acid pairs, this is a canonical way to include similarity.

A basis is orthonormal if it consists of states that are pairwise orthogonal. The basis $|1\rangle,|2\rangle, \ldots,|20\rangle$ is the standard orthonormal basis of $\mathfrak{A}$. To model amino acid pairs occurring in MSA column pairs, we need two copies $\mathfrak{A}_{1}$ and $\mathfrak{A}_{2}$ of $\mathfrak{A}$. Pairs are then represented as tensors $|i j\rangle=|i\rangle \otimes|j\rangle \in \mathfrak{A}_{1} \otimes \mathfrak{A}_{2} \quad(i, j=1,2, \ldots, 20)$ which are Kronecker products of the corresponding column vectors of dimension 20 . For $i, j=1,2, \ldots, 20$, the column vector 
$|i j\rangle$ has a dimension of 400 , where exactly that entry is equal to 1 that corresponds to the pair $(i, j)$. All other coefficients are equal to 0 .

The amino acid conservation of an MSA column pair is measured on grounds of its empirical amino acid distribution $\widehat{p}=\left(\widehat{p}_{i j}\right)_{i, j=1,2 \ldots .20}$, where $\widehat{p}_{i j}$ is the relative frequency of the pair of the $i$-th and the $j$-th amino acid in that column pair. If we choose a row of the MSA column pair by pure chance, we get the amino acid pair $(i, j)$ with probability $\widehat{p}_{i j}$ $(i, j=1,2, \ldots, 20)$. In Dirac notation this is expressed by the density operator

$$
\rho(\widehat{p})=\sum_{i, j=1}^{20} \widehat{p}_{i j}|i j\rangle\langle i j|
$$

The logarithm of $\rho(\widehat{p})$ (see Equation 5.1.1) is $\sum_{i, j=1}^{20} \log \widehat{p}_{i j}|i j\rangle\langle i j|$, where $\log p$ is set to 0 if $p=0$. This entails $-\rho(\widehat{p}) \log \rho(\widehat{p})=-\sum_{i, j=1}^{20} \widehat{p}_{i j} \log \widehat{p}_{i j}|i j\rangle\langle i j|$. Then, the entropy of the distribution $\widehat{p}$ equals the trace of the matrix $-\rho(\widehat{p}) \log \rho(\widehat{p})$ :

$$
\operatorname{tr}(-\rho(\widehat{p}) \log \rho(\widehat{p}))=-\sum_{i, j=1}^{20} \widehat{p}_{i j} \log \widehat{p}_{i j}
$$

Positive semi-definite operators of trace 1 are the most general form of density operators. For taking amino acid pair similarity into account when modeling MSA column pairs, the density operator $\rho(\widehat{p})$ of Equation 5.1.1 is swapped for

$$
\rho=\sum_{i, j=1}^{20} \widehat{p}_{i j}\left|\pi_{i j}\right\rangle\left\langle\pi_{i j}\right|
$$

where $\left|\pi_{i j}\right\rangle$ is a not necessarily orthonormal basis of states that represents the amino acids pairs $(i, j)$, for $i, j=1,2, \ldots, 20$. The corresponding Gram matrix

$$
\mathcal{A}=\left(\left\langle\pi_{i j} \mid \pi_{k l}\right\rangle\right)_{i, j, k, l=1,2, \ldots, 20}
$$

reflects predefined similarities between the 400 amino acid pairs.

For positive semi-definite operators of trace 1 like $\rho$ there is always an orthonormal basis $\left|\xi_{i j}\right\rangle(i, j=1,2, \ldots, 20)$ such that

$$
\rho=\sum_{i, j=1}^{20} q_{i j}\left|\xi_{i j}\right\rangle\left\langle\xi_{i j}\right|,
$$

where the eigenvalues $q_{i j} \geq 0$ sum up to one. Then, we calculate the von Neumann entropy 
of $\rho$ as

$$
\operatorname{VNE}(\rho)=\operatorname{tr}(-\rho \log \rho)=-\sum_{i, j=1}^{20} q_{i j} \log q_{i j}
$$

The difference between the Shannon entropy given by Equation 5.1.2 and the von Neumann entropy defined by Equation 5.1.6 is that the more similar the vectors $\left|\pi_{i j}\right\rangle$ appearing in Equation 5.1.3 are, the less is the von Neumann entropy compared with the Shannon entropy.

The predefined Gram matrix $\mathcal{A}$ (see Equation 5.1.4 does not fully determine the states $\left|\pi_{i j}\right\rangle$, for $i, j=1,2, \ldots, 20$. In order to be able to specify additional conditions for a consistent amino acid pair model, we need measurements as another key notion of quantum mechanics. To this end, let $P_{k l}=|k l\rangle\langle k l|$ be the orthogonal projection from $\mathfrak{A}_{1} \otimes \mathfrak{A}_{2}$ onto the subspace spanned by $|k l\rangle$ so that $P_{k l}|\xi\rangle=\langle k l \mid \xi\rangle|k l\rangle$, where $k, l=1,2, \ldots, 20$, and $|\xi\rangle \in \mathfrak{A}_{1} \otimes \mathfrak{A}_{2}$.

By definition, measuring a density matrix $\rho$ given by Equation 5.1 .3 with respect to the standard basis $|k l\rangle(k, l=1,2, \ldots, 20)$ results in the density matrix $\sum_{k, l=1}^{20} P_{k l} \rho P_{k l}$. This measurement determines the relative amino acid pair frequencies of the column pair under study. That is why it is reasonable to require for all $k, l=1,2, \ldots, 20$

$$
P_{k l} \rho P_{k l}=\widehat{p}_{k l}|k l\rangle\langle k l| \text {. }
$$

We ensure that a density operator $\rho$ satisfies these conditions having represented it with respect to the standard basis $|k l\rangle(k, l=1,2, \ldots, 20)$. The relative amino acid pair frequencies $\widehat{p}_{k l}$ have to appear on the main diagonal.

Amino acid pair distributions of MSA column pairs can be marginalized to get the amino acid distributions of the MSA columns concerned. Thus, we use the partial trace which is the analog way in quantum mechanics to observe the amino acid distributions of columns. Let $i$ and $j$ denote the amino acids of the first column, and $k$ and $l$ the amino acids of the second column. If $\rho=\sum_{i, j, k, l=1}^{20} \gamma_{i k j l}|i k\rangle\langle j l|$ is a density operator that describes the amino acid pair distribution of an MSA column pair including pair similarity, then the partial traces of $\rho$ over the first and the second column are:

$$
\operatorname{tr}_{1}(\rho)=\sum_{k, l=1}^{20}\left(\sum_{i=1}^{20} \gamma_{i k i l}\right)|k\rangle\left\langle l\left|, \quad \operatorname{tr}_{2}(\rho)=\sum_{i . j=1}^{20}\left(\sum_{k=1}^{20} \gamma_{i k j k}\right)\right| i\right\rangle\langle j|
$$

As we have explained in Section 3.2.1 partial traces of density operators are also density operators. Here, they involve amino acid similarity schemes that are "marginals" of the underlying amino acid pair similarity. The Algorithm 3 shows how to implement $\operatorname{tr}_{1}(\rho)$ and $\operatorname{tr}_{2}(\rho)$. 


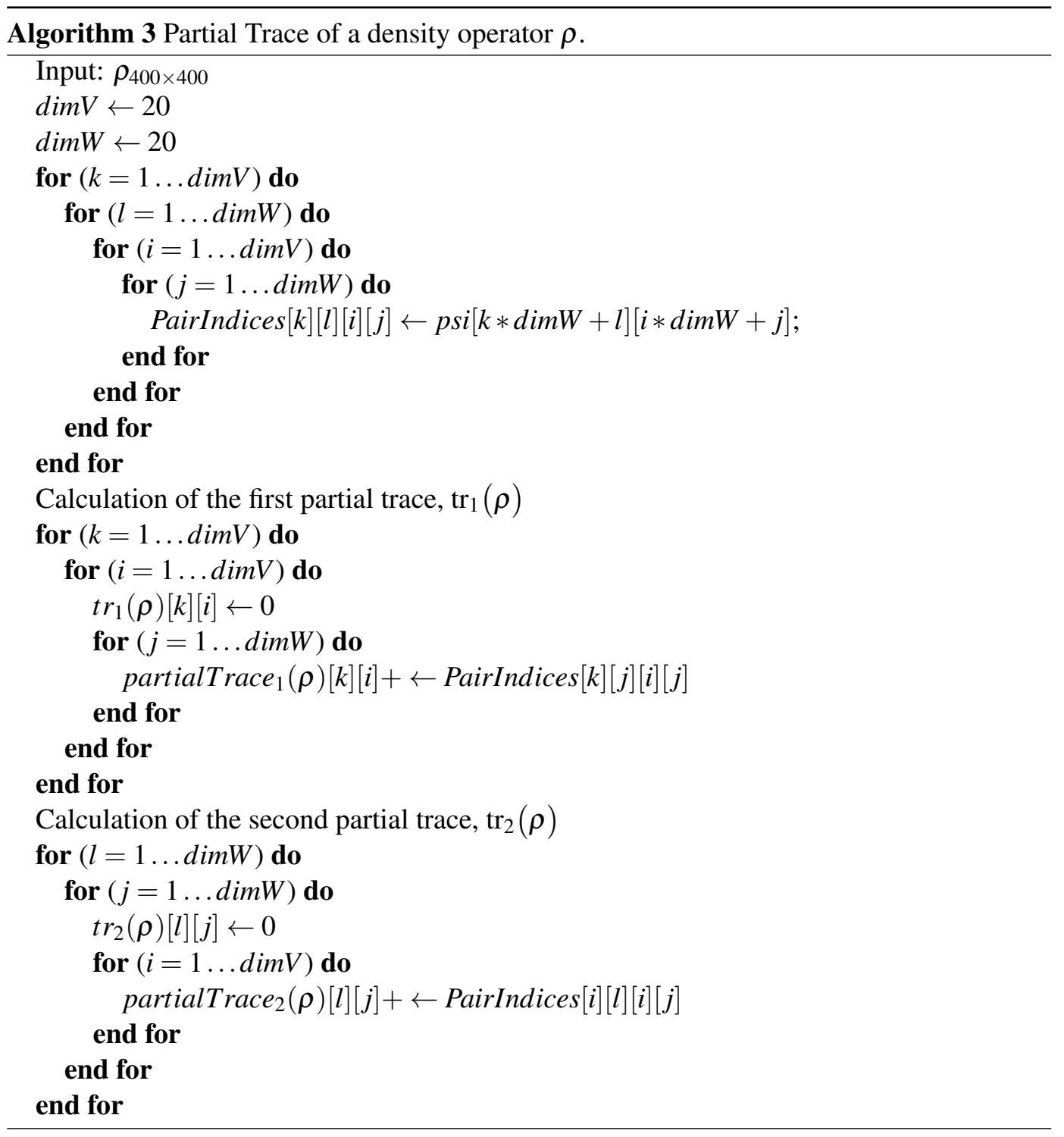




\subsubsection{Calculating the Gram matrices $\mathcal{A}_{\text {ent }}$ and $\mathcal{A}_{\text {sep }}$ to be plugged into Equation 5.1 .4}

We leverage the matrices $C_{\text {alt }}$ and $C_{\text {null }}$ (see Section 4.3.3 to calculate of the Gram matrices. The entries of the two matrices are frequencies of pair substitutions: $C_{\text {alt }}$ models the correlation signals between pair of amino acid pairs; $C_{\text {null }}$ reflects the background signals.

We define two significant pair substitution matrices $C_{\text {ent }}$ and $C_{\text {sep }}$ from $C_{\text {alt }}$ and $C_{\text {null }}$ which form the basis of our new metrics $\mathbb{Q}_{\text {ent }}$ and $\mathbb{Q}_{\text {sep }}$ (both metrics will be defined in Section 5.2). The intuition behind $C_{\text {ent }}$ is that BLOSUM62-based pair similarities are rescaled, whereas $C_{\text {sep }}$ is the basis for a new amino acid pair similarity.

$$
C_{\text {ent }}\left(\left(a_{i}, a_{j}\right),\left(a_{k}, a_{l}\right)\right)= \begin{cases}C_{\mathrm{alt}}\left(\left(a_{i}, a_{j}\right),\left(a_{k}, a_{l}\right)\right) & \text { if } \varphi_{\mathrm{ent}}\left(\left(a_{i}, a_{j}\right),\left(a_{k}, a_{l}\right)\right)=1 ; \\ 0 & \text { otherwise; }\end{cases}
$$

where $\varphi_{\text {ent }}\left(\left(a_{i}, a_{j}\right),\left(a_{k}, a_{l}\right)\right)=1$ if and only if either $\left(a_{i}, a_{j}\right)=\left(a_{k}, a_{l}\right)$ or the pair $\left(a_{i}, a_{k}\right)$ as well as the pair $\left(a_{j}, a_{l}\right)$ are BLOSUM62-similar and

$$
\begin{gathered}
\frac{C_{\mathrm{alt}}\left(\left(a_{i}, a_{j}\right),\left(a_{k}, a_{l}\right)\right)}{\sum_{i^{\prime}, j^{\prime}, k^{\prime}, l^{\prime}} C_{\mathrm{alt}}\left(\left(a_{i^{\prime}}, a_{j^{\prime}}\right),\left(a_{k^{\prime}}, a_{l^{\prime}}\right)\right)}>\frac{C_{\mathrm{null}}\left(\left(a_{i}, a_{j}\right),\left(a_{k}, a_{l}\right)\right)}{\sum_{i^{\prime}, j^{\prime}, k^{\prime}, l^{\prime}} C_{\mathrm{null}}\left(\left(a_{i^{\prime}}, a_{j^{\prime}}\right),\left(a_{k^{\prime}}, a_{l^{\prime}}\right)\right)} . \\
C_{\mathrm{sep}}\left(\left(a_{i}, a_{j}\right),\left(a_{k}, a_{l}\right)\right)= \begin{cases}C_{\mathrm{alt}}\left(\left(a_{i}, a_{j}\right),\left(a_{k}, a_{l}\right)\right) & \text { if } \varphi_{\text {sep }}\left(\left(a_{i}, a_{j}\right),\left(a_{k}, a_{l}\right)\right)=1 ; \\
0 & \text { otherwise; }\end{cases}
\end{gathered}
$$

where $\varphi_{\text {sep }}\left(\left(a_{i}, a_{j}\right),\left(a_{k}, a_{l}\right)\right)=1$ if and only if either $\left(a_{i}, a_{j}\right)=\left(a_{k}, a_{l}\right)$ or Equation 5.1 .10 is satisfied.

Let $C$ be either $C_{\text {ent }}$ or $C_{\text {sep }}$. We define

$$
B_{(g, h),(i, j)}=\frac{C_{(g, h),(i, j)}^{\alpha}}{\sqrt{\sum_{l, \kappa=1}^{20} C_{(l, \kappa),(i, j)}^{2 \alpha}}},
$$

where $((g, h),(i, j))$ range over all possible 160000 indices of pairs of amino acid pairs including the main diagonal, and $\alpha \in(0,1)$ was appropriately chosen.

To ensure positive semi-definiteness, we finally set

$$
\mathcal{A}=B^{T} B .
$$

That way we obtain $\mathcal{A}_{\text {ent }}$ as well as $\mathcal{A}_{\text {sep }}$. 


\subsubsection{Simultaneously ensuring Equations 5.1.3, 5.1.4, and 5.1.7}

Having chosen the kets $\left|\pi_{i j}\right\rangle$ that appear in Equation 5.1.3 so that the uniquely determined positive semi-definite square root $\sqrt{\rho}$ of $\rho$ equals $\sum_{i, j=1}^{20} \widehat{q}_{i j}\left|\pi_{i j}\right\rangle\langle i j|$, where $\widehat{q}=$ $\left(\widehat{q}_{i j}\right)_{i, j=1,2, \ldots, 20}$ represents the amino acid pair distribution we wish to plug into $\rho$, Equation 5.1 .3 is ensured. Moreover, we get

$$
\rho=\sum_{g, h, i, j=1}^{20} \sqrt{\widehat{q}_{g h}} \mathcal{A}_{(g, h),(i, j)} \sqrt{\widehat{q}_{i j}}|g h\rangle\langle i j|
$$

Therein, $\mathcal{A}$ is either equal to $\mathcal{A}_{\text {ent }}$ or equal to $\mathcal{A}_{\text {sep }}$. (Note, that Equation 5.1.14 corresponds to an approach due to Johansson et al. [86] for MSA columns rather than MSA column pairs.)

Equation 5.1.7 follows now from Equation 5.1.14 in a straightforward manner of swapping $\widehat{p}=\left(\widehat{p}_{i j}\right)_{i, j=1,2, \ldots, 20}$ for $\widehat{q}=\left(\widehat{q}_{i j}\right)_{i, j=1,2, \ldots, 20}$. Since the square root $(\sqrt{\rho})$ of $\rho$ is in particular symmetric, we get $\rho=\sum_{g, h, i, j=1}^{20} \sqrt{\widehat{q}_{g h}}\left\langle\pi_{g h} \mid \pi_{i j}\right\rangle \sqrt{\widehat{q}_{i j}}|g h\rangle\langle i j|$. Comparing the latter with Equation 5.1.14, Equation 5.1.4 follows.

\subsection{Defining two new metrics $\mathbb{Q}$ ent and $\mathbb{Q}_{\text {sep }}$}

In the Section 4.3.3 a pair $\left(\left(a_{i}, a_{j}\right),\left(a_{k}, a_{l}\right)\right)$ of amino acid pairs is called a formal dissimilar compensatory mutation, if the BLOSUM62-score of both $\left(a_{i}, a_{k}\right)$ and $\left(a_{j}, a_{l}\right)$ is negative.

To define our first new metric $\mathbb{Q}_{\text {ent }}$ for a certain column pair of a given MSA, let $\widehat{q}=$ $\widehat{p} \cdot D_{\text {CompMut }}$, where $D_{\text {CompMut }}$ is the $400 \times 400$ doubly stochastic matrix defined in Section 4.3 .3 , and $\hat{p}$ is the empirical pair distribution of these two columns. Having determined $\rho_{\text {ent }}$ by Equation 5.1.14, where $\mathcal{A}$ is swapped for $\mathcal{A}_{\text {ent }}$, our first metric is

$$
\mathbb{Q}_{\mathrm{ent}}=\operatorname{QJSD}\left(\rho_{\mathrm{ent}} \| \rho(\widehat{p})\right) \text {. }
$$

It is the quantum Jensen-Shannon divergence of an entangled density matrix that incorporates rescaled amino acid pair similarity as well as dissimilar compensatory mutations with a separable density operator representing the empirical amino acid pair frequencies.

Our second new metric $\mathbb{Q}_{\text {sep }}$ replaces the $\mathbb{U}$-metric introduced in the Section 4.1 by a measure that integrates a new amino acid pair similarity score into the framework of quantum Jensen-Shannon divergence. To this end, let $\rho_{\text {sep }}$ be defined by Equation 5.1.14, where $\widehat{q}$ is equal to the empirical amino acid pair distribution $\widehat{p}$ of the column pair under study, and $\mathcal{A}=\mathcal{A}_{\text {sep }}$. Then $\mathbb{Q}_{\text {sep}}$-metric is defined as

$$
\mathbb{Q}_{\text {sep }}=\operatorname{QJSD}\left(\operatorname{tr}_{1}\left(\rho_{\text {sep }}\right) \| \operatorname{tr}_{2}\left(\rho_{\text {sep }}\right)\right) .
$$




\subsection{Discussion}

Grosse et al. observed in [87] that the Jensen-Shannon divergence (JSD) can be interpreted as mutual information between two (or more) random sources in a special setting particularly appropriate to discriminate between these sources. This is what we need when it comes to predicting important protein sites in an MSA-based approach. It might explain the findings of Capra and Singh [88] on the predictive power of JSD. These two articles encouraged us to utilize quantum Jensen-Shannon divergence (QJSD) in this thesis. As a side effect, a normalization is not necessary, since quantum Jensen-Shannon divergence, like its classical counterpart, ranges over the real interval $[0,1]$.

Several studies have confirmed that detecting coupled MSA-columns is extremely useful in the prediction of important protein sites (see e.g. [4, 8, 9, 15,-24]). When using informationtheoretic metrics, there is no doubt that it is reasonable to incorporate amino acid pair dissimilarity as well as amino acid similarity in a consistent way such that similarity decreases entropy, whereas dissimilarity increases it. This kind of consistency is important, since entropy is the fundamental building block for most of those metrics. In particular, the Jensen-Shannon divergence between two probability mass functions $p$ and $q$ equals $\mathbb{H}(1 / 2(p+q))-1 / 2(\mathbb{H}(p)+\mathbb{H}(q))$.

In the Section 4.3 we have presented an amino acid pair dissimilarity model for compensatory mutations. A doubly stochastic matrix transforms the empirical amino acid pair distribution of a column pair. Rescaled pair similarity of BLOSUM62-similar pairs is to capture an aspect of coupled MSA column pairs orthogonal to the phenomenon of dissimilar compensatory mutations. It models the amino acid pair transition preferences within those column pairs on the average. As suggested by Caffrey et al. [89] as well as Johansson et al. [86], it is promising to incorporate them within the framework of quantum information theory. Therein, density matrices replace probability mass functions. The counterpart of the entropy of a probability mass function is the von Neumann entropy (VNE) of a density matrix (see Equation 5.1.6. QJSD corresponds then exactly to JSD.

The challenge was to complement the model presented in the Section 4.3 by additionally incorporating amino acid pair similarity in a way that the two effects interfere but do not interact. We model the 400 amino acid pairs by means of 400 not necessarily orthogonal tensors spanning the tensor product of two copies of a 20-dimensional Hilbert space. This provides us with the opportunity to utilize the notion of entanglement, which in turn is a major resource of quantum information. Moreover, we are in a position to make use of partial traces, which play the role of the marginals in the classical case. Pair similarity is reflected by means of the Gram matrix of these tensors (see Equation 5.1.4). To ensure positive definiteness, which is a key property of density matrices, we used transitivity of similarity (see Equation 5.1.13). Since there is no transitivity of dissimilarity, we kept dissimilarity apart from that Gram matrix. Instead, we carried over our previously defined BLOSUM62-dissimilarity model which was used to develop $\mathbb{U}_{D(\alpha)}$-metric (see Section 4.3). 
Gram matrix and transformed amino acid pair distribution are joined together by means of Equation 5.1.14 in the final step of our density matrix design. That way we minimize the interaction between the two effects of dissimilarity and similarity.

In order to eliminate the noise and to define an MSA-dependent threshold for significant column pairs, we followed the line of the Section 4.2. The MSA-specific statistical model presented there seems to be of universal applicability. The same is true for the connectivity degree model introduced in the Section 4.2.2. Combining them results in a reliable and robust method to determine significant residue sites. 


\section{Results}

In this chapter, we will analyze important sites of the human epidermal growth factor receptor (EGFR) protein and glucokinase (GCK) protein in order to demonstrate the functionality of our both classical and quantum information theory based methods. We have selected both proteins because their functionally and structurally important sites have been experimentally well studied [29-40].

EGFR is a member of the ErbB (Erythroblastic Leukemia Viral Oncogene Homolog) family receptors. Signaling through this receptor is a highly conserved mechanism from nematode to humans involved in numerous processes, including proliferation, cell fate determination, and tissue specification [90]. Furthermore, several studies have implicated that mutations resulting in misregulation of the activity or action of EGFR led to multiple cancers, including those of the brain, lung, mammary gland, and ovary [29-32].

GCK is a monomeric enzyme catalyzing phosphorylation of glucose to glucose-6phosphate, which is the first step in the utilization of glucose, at physiological glucose concentration in pancreas and liver. Given the fact that GCK displays low affinity for glucose, it acts as a glucose sensor playing an important role in the regulation of carbohydrate metabolism. Mutations of the GCK gene can lead to maturity onset diabetes of the young (MODY) characterized by an autosomal dominant mode of inheritance and onset early adulthood [37], or familial hyperinsulinemic hypoglycemia type 3 (HHF), which is common cause of persistent hypoglycemia in infancy [91].

In Section 6.1, we will start with the introducing the structurally or functionally important residue sites of these two human proteins. After that, in Section 6.2 we will go on analyzing both proteins in detail with $\mathbb{U}$-metric and $\mathbb{U}_{D(\alpha)}$-metric presented in Chapter 4. In addition, we will compare the functionality of these metrics with each other to evaluate their performance statistically. In Section 6.3, we will predict the important sites of EGFR and GCK proteins applying our quantum information theory based metrics $\mathbb{Q}_{\text {ent }}$ and $\mathbb{Q}_{\text {sep }}$ presented in the Chapter 5 and analyze the predicted sites in detail. Finally, in Section 6.4 we will make a comparison between the results of both classical and quantum information theory based methods.

We have previously published most of this chapter in [4] and [5] (see Appendix A 9.1 and $\mathrm{B} 9.2$. 


\subsection{Essential sites of human EGFR and GCK protein}

To evaluate the significant residue sites detected by our classical and quantum information theory based methods, we have investigated essential sites of human EGFR (pdb entry $2 \mathrm{~J} 6 \mathrm{M}$ ) and GCK (pdb entry 1V4S) proteins. The essential sites of both proteins have been assigned into three main categories: i) the nsSNP positions and their adjacent sites; ii) residue positions which are located at or near active sites, allosteric sites, or binding sites; iii) residue positions which are nearby strictly conserved sites. Here, we have used "nearby" definition of Nussinov et al. [62] and defined two residues as in contact or adjacent when the distance between their major carbon atoms is less than $6 \AA$ (for the distance calculation see the Section 2.5.4). We have defined positions which are nearby nsSNPs as essential, because several of them are also adjacent to active sites, allosteric sites, binding sites, or strictly conserved sites. Thus, we refer to a significant residue site as "functionally or structurally important" if it falls into one of these categories of essential sites.

\subsubsection{MSAs and 3-D structures of human EGFR and GCK protein}

We gathered the related MSAs of both proteins from the HSSP database (see Section 2.5.2] [54], and their corresponding 3-D structure information were downloaded from PDB database (see Section 2.5.1) [2] which are necessary to determine the distance between residues.

The human EGFR protein has a chain length of 306 residues and its related MSA contains 1551 sequences. Likewise, the human GCK protein has a chain length of 448 residues and its related MSA contains 785 sequences. In Figure 6.1 and 6.2, we illustrate positions of residues in both proteins with corresponding residue sequence number in PDB files.

\subsection{Applying the $\mathbb{U}$-metric and the $\mathbb{U}_{D(\alpha)}$-metric for the human EGFR and GCK protein}

We apply our classical information theory based method to predict functionally or structurally important residue positions in two steps. First, we utilize the new MSA-specific statistical method presented in the Section 4.2 for the identification of significant MSA column pairs with respect to either of the two metrics $\mathbb{U}$ and $\mathbb{U}_{D(\alpha)}$. Assuming that $M$ is the MSA under study, these pairs are annotated as $(\mathbb{U}, M)$-significant and $\left(\mathbb{U}_{D(\alpha)}, M\right)$-significant, respectively. Second, we utilized the connectivity degree of a residue site (see in the Section 4.2.2). Recall that the connectivity degree of a residue site indicates the number of its significant coupled mutation pairs. In this case, a site is called (U,M)-significant when the frequency of occurrence of this site in the set of $(\mathbb{U}, M)$-significant pairs exceeds the 90 -th percentile. Having defined the concept of a $\left(\mathbb{U}_{D(\alpha)}, M\right)$-significant site analogously, a site 


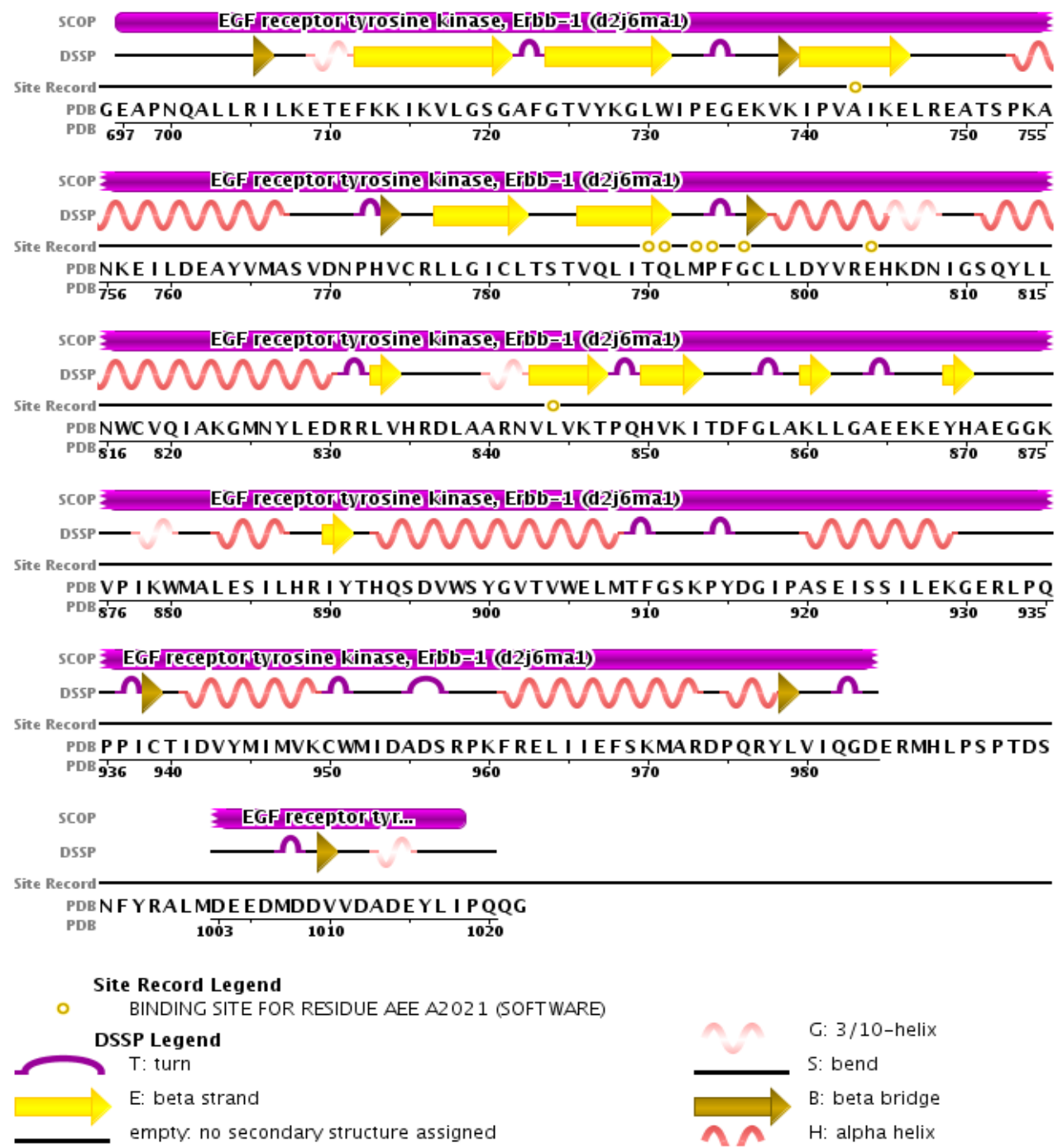

Figure 6.1: The sequence of the human EGFR protein (PDB-Entry 2J6M) (image from http://www.rscb.org [2], 05.08.2013).

is defined as CMF-significan 8 with respect to $M$, when it is either $(\mathbb{U}, M)$-significant or $\left(\mathbb{U}_{D(\alpha)}, M\right)$-significant.

\footnotetext{
${ }^{8}$ We defined the predicted significant sites together as CMF-significant due to our corresponding publication

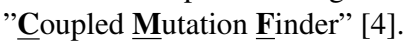




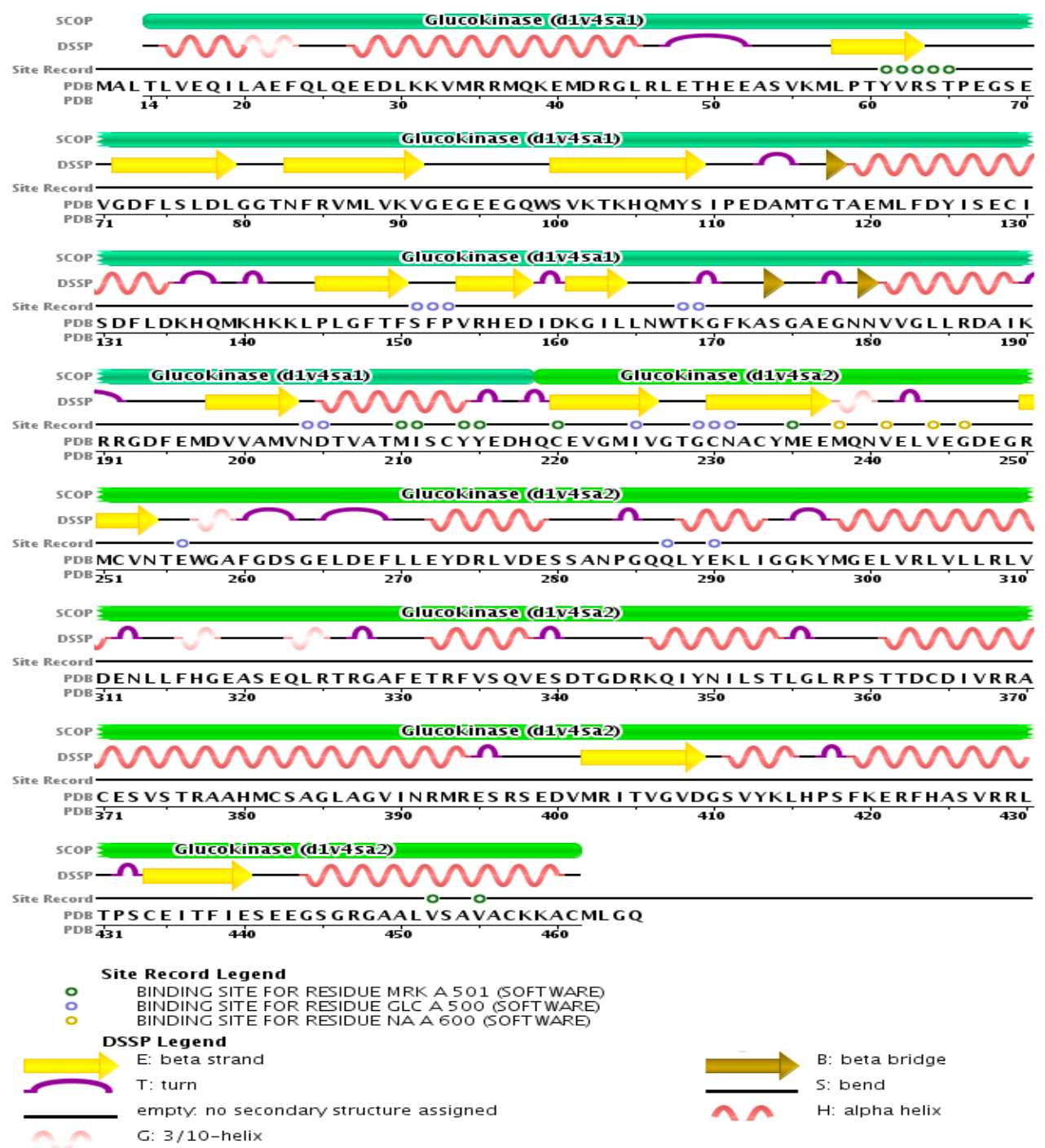

Figure 6.2: The sequence of the human GCK protein (PDB-Entry 1V4S) (image from http://www.rscb.org [2], 05.08.2013).

We analyzed both proteins with a false discovery rate (FDR) of $1 \%$. For EGFR, we defined a total of 14339 out of 26079 non-conserved column pairs as significant. 11365 of these significant pairs are detected as $(\mathbb{U}, M)$-significant and 3798 pairs are observed as $\left(\mathbb{U}_{D(\alpha)}, M\right)$ significant. Only 824 EGFR pairs are significant with respect to both metrics. On the other 
hand, for GCK, we identified a total of 32654 out of 69645 non-conserved column pairs as significant where 18106 of them are $\mathbb{U}$-significant and 16241 are $\mathbb{U}_{D(1)}$-significant. Moreover, 1693 pairs are defined as significant for both $\mathbb{U}$ and $\mathbb{U}_{D(1)}$-significant.

Applying the connectivity degree technique, we identified 22 and 36 residue positions as $\mathbb{U}$-significant for human EGFR and GCK proteins, respectively. Additionally, 21 positions of EGFR and 36 positions of GCK were detected as $\mathbb{U}_{D(1)}$-significant. Finally, a total of 43 sites of EGFR and 72 of GCK were found as CMF-significant, and predicted as of structural or functional importance (see Table 6.1 and 6.2. However, there have been no residue sites detected as significant by both metrics. 


\begin{tabular}{|c|c|c|c|c|c|c|c|c|}
\hline & Residue & Position & Connectivity Degree & Detected by & Residue & Position & Connectivity Degree & Detected by \\
\hline & $\mathrm{S}$ & 720 & 170 & U-metric & $\mathrm{Q}$ & 849 & 173 & U-metric \\
\hline & $\mathrm{T}$ & 725 & 178 & $\mathbb{U}$-metric & K & 860 & 113 & $\mathbb{U}_{D(\alpha)^{-m e t r i c}}$ \\
\hline & $\mathrm{Y}$ & 727 & 169 & $\mathbb{U}$-metric & G & 863 & 170 & U-metric \\
\hline & E & 746 & 168 & $\mathbb{U}$-metric & E & 868 & 170 & $\mathbb{U}$-metric \\
\hline & $\mathrm{A}$ & 755 & 177 & $\mathbb{U}$-metric & E & 872 & 170 & U-metric \\
\hline & $\mathrm{N}$ & 756 & 192 & $\mathbb{U}$-metric & V & 876 & 173 & $\mathbb{U}$-metric \\
\hline & $\mathrm{E}$ & 758 & 121 & $\mathbb{U}_{D(\alpha)}$-metric & K & 879 & 109 & $\mathbb{U}_{D(\alpha)}$-metric \\
\hline & I & 759 & 123 & $\mathbb{U}_{D(\alpha)^{-}}$-metric & $\mathrm{Y}$ & 891 & 120 & $\mathbb{U}_{D(\alpha)}$-metric \\
\hline & $\mathrm{Y}$ & 764 & 174 & $\mathbb{U}$-metric & $\mathrm{T}$ & 892 & 175 & $\mathbb{U}_{D(\alpha)^{-m e t r i c}}$ \\
\hline & M & 766 & 104 & $\mathbb{U}_{D(\alpha)^{-}}$-metric & $S$ & 899 & 97 & $\mathbb{U}_{D(\alpha)}$-metric \\
\hline & A & 767 & 173 & $\mathbb{U}$-metric & $\mathrm{Y}$ & 900 & 119 & $\mathbb{U}_{D(\alpha)}$-metric \\
\hline & $\mathrm{H}$ & 773 & 125 & $\mathbb{U}_{D(\alpha)}$-metric & $\mathrm{T}$ & 909 & 158 & $\mathbb{U}_{D(\alpha)}$-metric \\
\hline & Q & 791 & 198 & $\mathbb{U}_{D(\alpha)^{-m e t r i c}}$ & $S$ & 912 & 174 & $\mathbb{U}$-metric \\
\hline & $\mathrm{D}$ & 800 & 102 & $\mathbb{U}_{D(\alpha)}$-metric & K & 913 & 170 & $\mathbb{U}$-metric \\
\hline & $\mathrm{N}$ & 816 & 176 & $\mathbb{U}$-metric & $\mathrm{D}$ & 916 & 181 & $\mathbb{U}$-metric \\
\hline & $\mathrm{V}$ & 819 & 177 & $\mathbb{U}$-metric & A & 920 & 172 & $\mathbb{U}$-metric \\
\hline - & Q & 820 & 147 & $\mathbb{U}_{D(\alpha)}$-metric & $\mathrm{E}$ & 922 & 188 & $\mathbb{U}_{D(\alpha)}$-metric \\
\hline & G & 824 & 99 & $\mathbb{U}_{D(\alpha)}$-metric & $\mathrm{S}$ & 924 & 176 & $\mathbb{U}$-metric \\
\hline & $\mathrm{E}$ & 829 & 101 & $\mathbb{U}_{D(\alpha)^{-m e t r i c}}$ & $\mathrm{E}$ & 931 & 171 & $\mathbb{U}$-metric \\
\hline & D & 830 & 95 & $\mathbb{U}_{D(\alpha)}$-metric & $\mathrm{R}$ & 932 & 95 & $\mathbb{U}_{D(\alpha)}$-metric \\
\hline & K & 846 & 183 & $\mathbb{U}$-metric & M & 947 & 106 & $\mathbb{U}_{D(\alpha)^{-m e t r i c}}$ \\
\hline & $\mathrm{T}$ & 847 & 164 & $\mathbb{U}_{D(\alpha)}$-metric & & & & \\
\hline
\end{tabular}

Table 6.1: 43 CMF-significant residue sites found by $\mathbb{U}$ and $\mathbb{U}_{D(\alpha)}$-metric in human EGFR protein. 


\begin{tabular}{|c|c|c|c|c|c|c|c|}
\hline Residue & Position & Connectivity Degree & Detected by & Residue & Position & Connectivity Degree & Detected by \\
\hline $\mathrm{L}$ & 25 & 229 & U-metric & G & 264 & 258 & U-metric \\
\hline M & 34 & 238 & $\mathbb{U}$-metric & E & 265 & 250 & $\mathbb{U}$-metric \\
\hline $\mathrm{R}$ & 36 & 238 & $\mathbb{U}$-metric & $\mathrm{L}$ & 266 & 246 & $\mathbb{U}$-metric \\
\hline $\mathrm{E}$ & 40 & 302 & $\mathbb{U}_{D(\alpha)^{- \text {metric }}}$ & $\mathrm{D}$ & 267 & 246 & U-metric \\
\hline $\mathrm{T}$ & 60 & 305 & $\mathbb{U}_{D(\alpha)^{-m e t r i c}}$ & $\mathrm{E}$ & 268 & 242 & $\mathbb{U}$-metric \\
\hline $\mathrm{R}$ & 63 & 289 & $\mathbb{U}$-metric & $\mathrm{L}$ & 271 & 286 & $\mathbb{U}_{D(\alpha)}$-metric \\
\hline $\mathrm{T}$ & 65 & 213 & $\mathbb{U}$-metric & $\mathrm{S}$ & 281 & 286 & $\mathbb{U}_{D(\alpha)}$-metric \\
\hline $\mathrm{E}$ & 67 & 286 & $\mathbb{U}_{D(\alpha)}$-metric & $\mathrm{N}$ & 283 & 321 & $\mathbb{U}_{D(\alpha)}$-metric \\
\hline $\mathrm{T}$ & 82 & 318 & $\mathbb{U}_{D(\alpha)}$-metric & Q & 286 & 238 & $\mathbb{U}$-metric \\
\hline $\mathrm{N}$ & 83 & 312 & $\mathbb{U}_{D(\alpha)}$-metric & Q & 287 & 332 & $\mathbb{U}_{D(\alpha)}$-metric \\
\hline G & 92 & 224 & $\mathbb{U}$-metric & G & 294 & 265 & $\mathbb{U}_{D(\alpha)}$-metric \\
\hline $\mathrm{H}$ & 105 & 253 & $\mathbb{U}$-metric & $\mathrm{E}$ & 300 & 346 & $\mathbb{U}_{D(\alpha)}$-metric \\
\hline M & 107 & 276 & $\mathbb{U}$-metric & $\mathrm{E}$ & 331 & 241 & $\mathbb{U}$-metric \\
\hline $\mathrm{F}$ & 123 & 278 & $\mathbb{U}_{D(\alpha)^{- \text {metric }}}$ & $\mathrm{T}$ & 332 & 240 & $\mathbb{U}_{D(\alpha)}$-metric \\
\hline $\mathrm{C}$ & 129 & 217 & $\mathbb{U}$-metric & $\mathrm{R}$ & 333 & 223 & $\mathbb{U}$-metric \\
\hline $\mathrm{F}$ & 133 & 230 & $\mathbb{U}_{D(\alpha)^{- \text {metric }}}$ & Q & 337 & 221 & $\mathbb{U}$-metric \\
\hline $\mathrm{F}$ & 148 & 234 & $\mathbb{U}_{D(\alpha)^{-}}$-metric & $\mathrm{E}$ & 339 & 264 & $\mathbb{U}_{D(\alpha)}$-metric \\
\hline $\mathrm{T}$ & 149 & 277 & $\mathbb{U}_{D(\alpha)^{-m e t r i c}}$ & $\mathrm{D}$ & 341 & 299 & $\mathbb{U}_{D(\alpha)}$-metric \\
\hline $\mathrm{F}$ & 152 & 258 & $\mathbb{U}_{D(\alpha)^{-m e t r i c}}$ & G & 343 & 235 & U-metric \\
\hline $\mathrm{H}$ & 156 & 334 & $\mathbb{U}_{D(\alpha)}$-metric & $\mathrm{D}$ & 363 & 300 & $\mathbb{U}_{D(\alpha)}$-metric \\
\hline $\mathrm{F}$ & 171 & 243 & $\mathbb{U}_{D(\alpha)^{-}}$-metric & $\mathrm{N}$ & 391 & 224 & $\mathbb{U}$-metric \\
\hline $\mathrm{N}$ & 180 & 320 & $\mathbb{U}_{D(\alpha)^{-}}$-metric & $\mathrm{E}$ & 395 & 270 & U-metric \\
\hline $\mathrm{R}$ & 186 & 257 & $\mathbb{U}_{D(\alpha)}$-metric & $\mathrm{S}$ & 396 & 265 & U-metric \\
\hline $\mathrm{T}$ & 206 & 297 & $\mathbb{U}_{D(\alpha)^{-m e t r i c}}$ & $\mathrm{~T}$ & 405 & 264 & U-metric \\
\hline $\mathrm{T}$ & 209 & 292 & $\mathbb{U}_{D(\alpha)}$-metric & $\mathrm{S}$ & 411 & 315 & $\mathbb{U}_{D(\alpha)}$-metric \\
\hline $\mathrm{C}$ & 213 & 263 & $\mathbb{U}$-metric & $\mathrm{K}$ & 414 & 318 & $\mathbb{U}_{D(\alpha)}$-metric \\
\hline $\mathrm{E}$ & 216 & 227 & U-metric & S & 418 & 236 & $\mathbb{U}$-metric \\
\hline D & 217 & 241 & $\mathbb{U}_{D(\alpha)^{- \text {metric }}}$ & $\mathrm{F}$ & 419 & 226 & $\mathbb{U}_{D(\alpha)}$-metric \\
\hline E & 221 & 249 & $\mathbb{U}$-metric & $\mathrm{R}$ & 422 & 252 & $\mathbb{U}$-metric \\
\hline $\mathrm{T}$ & 228 & 282 & $\mathbb{U}_{D(\alpha)^{- \text {metric }}}$ & $\mathrm{H}$ & 424 & 250 & U-metric \\
\hline $\mathrm{E}$ & 236 & 351 & $\mathbb{U}_{D(\alpha)^{-m e t r i c}}$ & $\mathrm{~T}$ & 431 & 253 & U-metric \\
\hline V & 244 & 240 & $\mathbb{U}$-metric & S & 433 & 224 & U-metric \\
\hline $\mathrm{R}$ & 250 & 225 & U-metric & $\mathrm{C}$ & 434 & 227 & U-metric \\
\hline $\mathrm{F}$ & 260 & 237 & $\mathbb{U}_{D(\alpha)}$-metric & I & 439 & 221 & $\mathbb{U}$-metric \\
\hline G & 261 & 214 & U-metric & E & 442 & 319 & $\mathbb{U}_{D(\alpha)}$-metric \\
\hline D & 262 & 295 & $\mathbb{U}_{D(\alpha)}$-metric & $\mathrm{E}$ & 443 & 315 & $\mathbb{U}_{D(\alpha)}$-metric \\
\hline
\end{tabular}

Table 6.2: 72 CMF-significant residue sites found by $\mathbb{U}$ and $\mathbb{U}_{D(\alpha)}$-metric in human GCK protein. 


\subsubsection{Position analysis of the Human Epidermal Growth Factor Receptor (EGFR) protein}

In order to detect essential mutation positions in the corresponding sequence of human EGFR protein, we determined altogether 43 CMF-significant residue sites (see Table 6.1). 15 of these significant residue sites have been verified as nsSNP sites through the Ensembl database annotation and they are illustrated in Figure 6.3 .

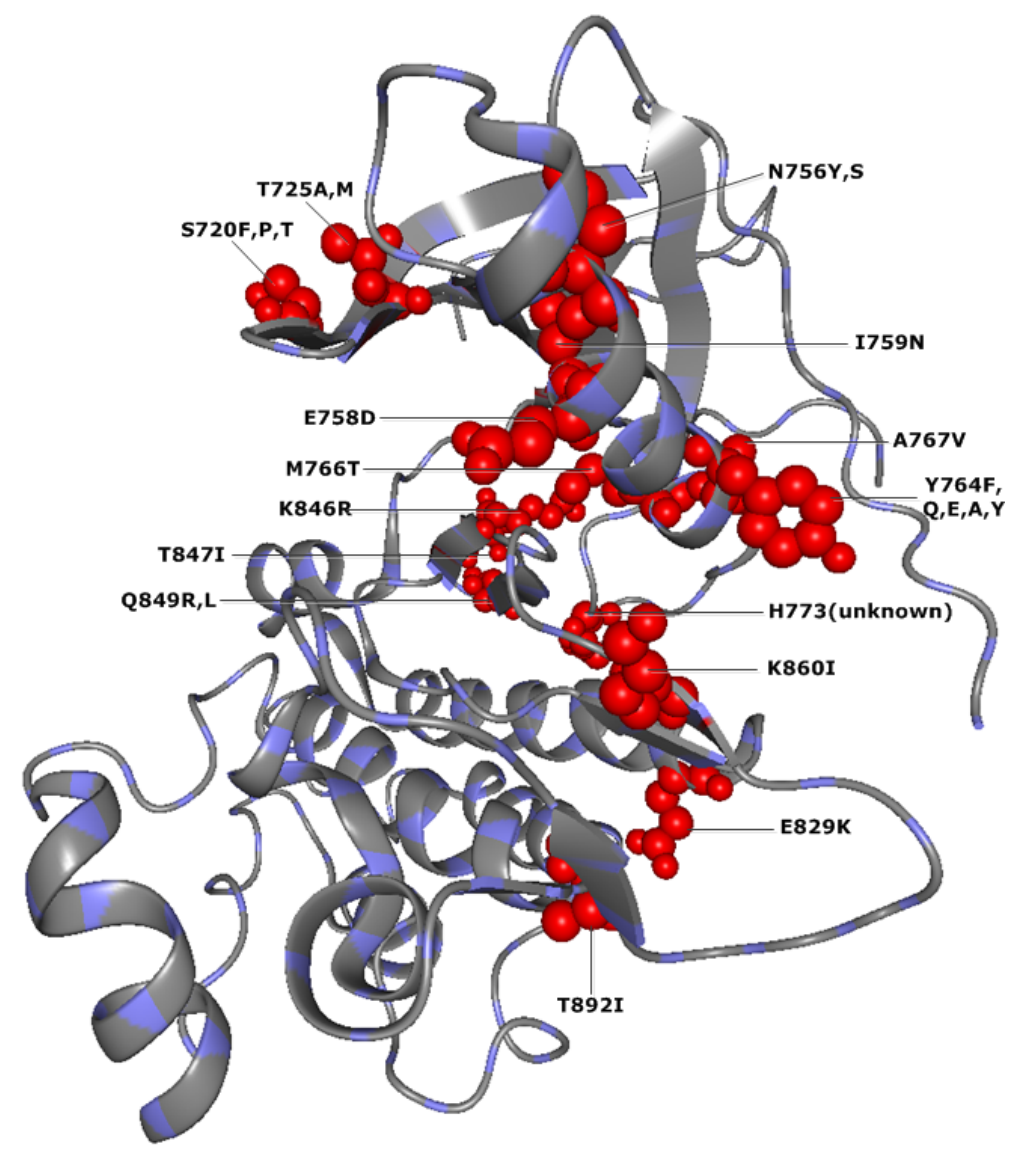

Figure 6.3: CMF-significant nsSNP positions in human EGFR protein (PDB-Entry 2J6M). The red spheres correspond to structural localization of 15 different CMFsignificant nsSNP positions in the EGFR protein.

Additionally, the CMF-significant sites E746, Q791, and four of the nsSNP positions (I759,Y764,M766 and K846) are also in contact with critical active site regions for the gefitinib binding site in the wild type EGFR kinase [30,33] (see Figure 6.4]). 


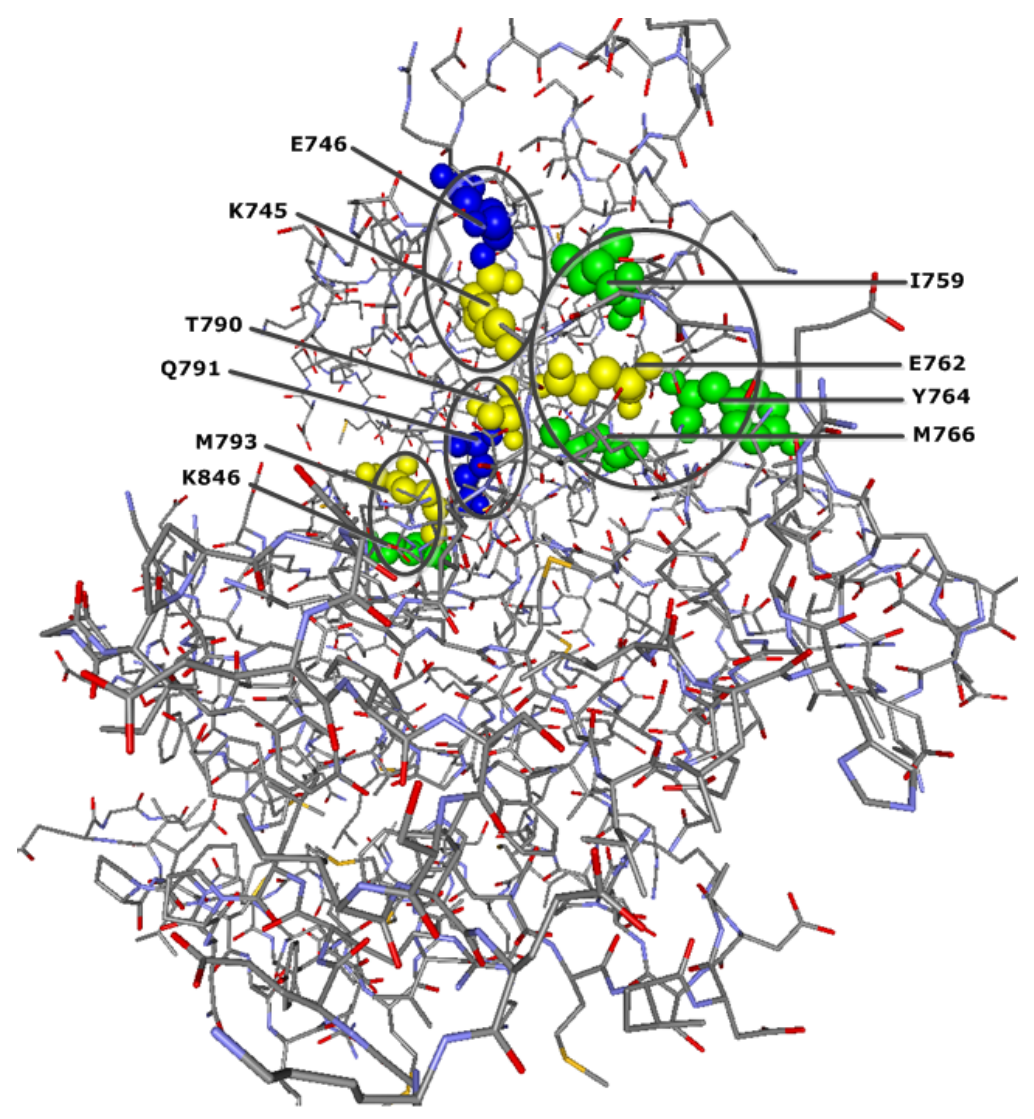

Figure 6.4: CMF-significant residue positions are in contact with gefitinib binding sites in human EGFR protein (PDB-Entry 2J6M ). Yellow spheres denote positions of the gefitinib binding sites in the wild type kinase. Blue spheres show the localization of CMFsignificant adjacent residue positions which are in contact with these binding sites. Additionally, the CMF-significant sites I759, Y764, M766 and K846, shown with green spheres, are already described as nsSNP positions and they are also in contact with gefitinib binding sites E762 and M793, respectively. Circles indicate clusters of gefitinib binding sites and their significant adjacent sites.

Moreover, we observed that 17 further CMF-significant positions are essential sites (see Table 6.3). In total, we have established for EGFR protein the importance of 34 out of 43 CMF-significant residue sites via different resources [30, 33, 39, 40].

\begin{tabular}{|c|l|l|}
\hline CMF-significant essential sites & Nearby nsSNPs or strictly conserved sites & Reference \\
\hline$Y 727$ & $726^{\mathbf{c}} 743^{\mathbf{c}}$ & - \\
\hline \multicolumn{3}{|c|}{ Continued on next page } \\
\hline
\end{tabular}


Table 6.3 - continued from previous page

\begin{tabular}{|c|l|l|}
\hline CMF-significant essential sites & Nearby nsSNPs or strictly conserved sites & References \\
\hline$H 755$ & $756^{\mathbf{s}}, 758^{\mathbf{s}}$ & \\
$D 800$ & $798^{\mathbf{c}}$ & \\
$G 824$ & $773^{\mathbf{s}}$ & \\
$D 830$ & $829^{\mathbf{s}}$ & \\
$E 868$ & $892^{\mathbf{s}}$ & {$[40]$} \\
$E 872$ & $873^{\mathbf{s}}$ & - \\
$V 876$ & $877^{\mathbf{c}}$ & - \\
$K 879$ & $877^{\mathbf{c}}, 880^{\mathbf{c}}$ & - \\
$Y 891$ & $892^{\mathbf{s}}, 895^{\mathbf{c}}$ & - \\
$S 899$ & $880^{\mathbf{c}}, 896^{\mathbf{c}}, 898^{\mathbf{c}}, 901^{\mathbf{c}}$ & - \\
$Y 900$ & $898^{\mathbf{c}}, 901^{\mathbf{c}}$ & - \\
$T 909$ & $906^{\mathbf{c}}, 936^{\mathbf{c}}$ & - \\
$S 912$ & $906^{\mathbf{c}}, 936^{\mathbf{c}}$ & - \\
$K 913$ & $914^{\mathbf{c}}$ & - \\
$D 916$ & $914^{\mathbf{c}}$ & \\
$M 947$ & $901^{\mathbf{c}}, 950^{\mathbf{c}}$ & - \\
\hline
\end{tabular}

Table 6.3: CMF-significant essential sites in human EGFR protein, which are nearby either nsSNPs or strictly conserved sites. ${ }^{\mathbf{s}}$ : non-synonymous snp site, ${ }^{\mathbf{c}}$ : strictly conserved site.

Although the vast majority of CMF-significant sites are verified to be structurally or functionally important in human EGFR protein, nine CMF-significant sites do not overlap with essential sites. The reason for the high connectivity degree of these unconfirmed significant sites and their role in the EGFR protein is unclear. 


\subsubsection{Position analysis of the Human Glucokinase (GCK) protein}

Applying our both metrics, we determined $72 \mathrm{CMF}$-significant residue sites to be structurally or functionally important in human GCK protein (see Table 6.2). 16 of these significant residue positions are related to disease associated nsSNP positions [34, 36, 39, 40] (see Figure 6.5.

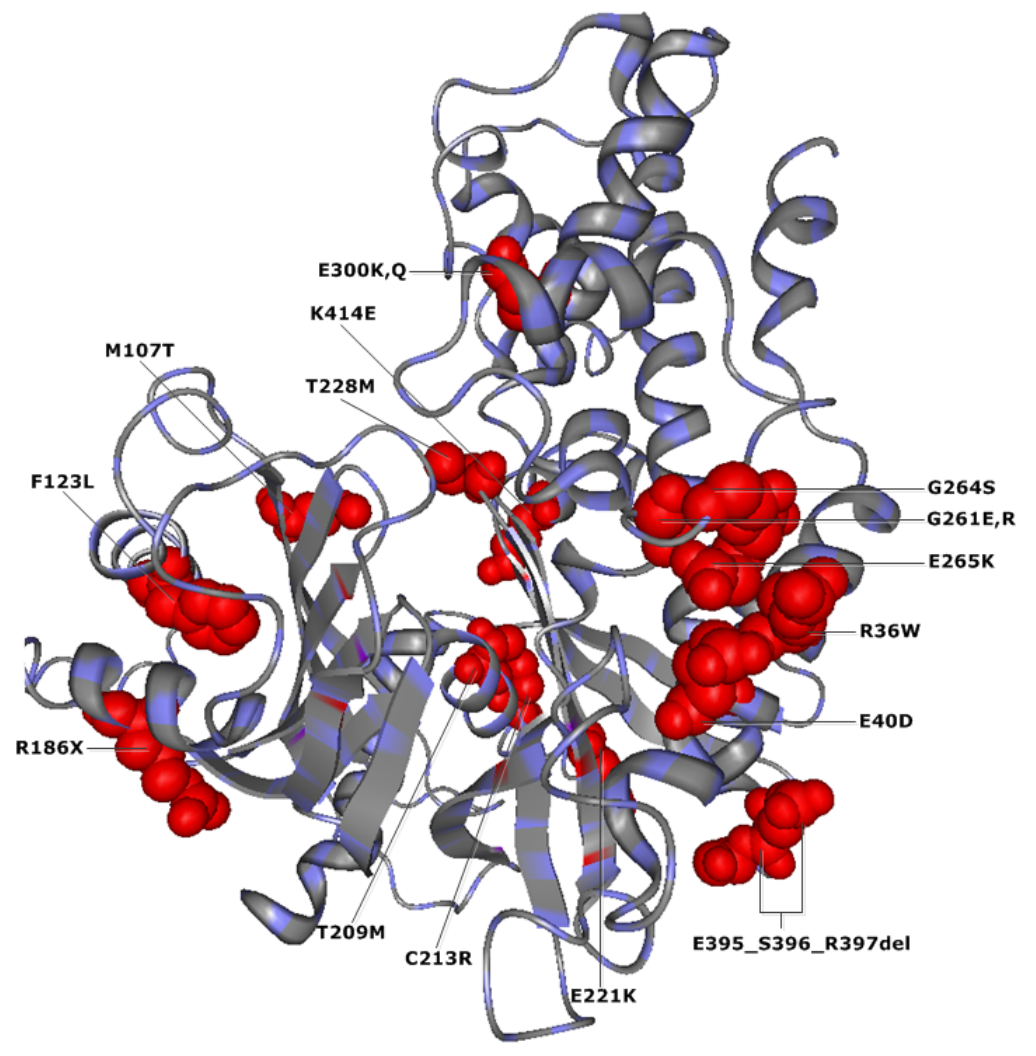

Figure 6.5: CMF-significant nsSNP positions in human GCK protein (PDB-Entry 1V4S). Red spheres show the structural localization of 16 different CMF-significant nsSNP positions in the GCK protein.

Furthermore, nine significant sites are found to be in contact with allosteric sites in the GCK protein structure. Among these sites, the R63 is also allosteric site by itself [37] and T209, C213 and E221 overlap with nsSNP regions (see Figure 6.6B). Moreover, the five significant sites T149, F171, T206, Q287, and G294 interact with glucose binding sites K169, D204, N205, and E290 [37] (see Figure 6.6A). 

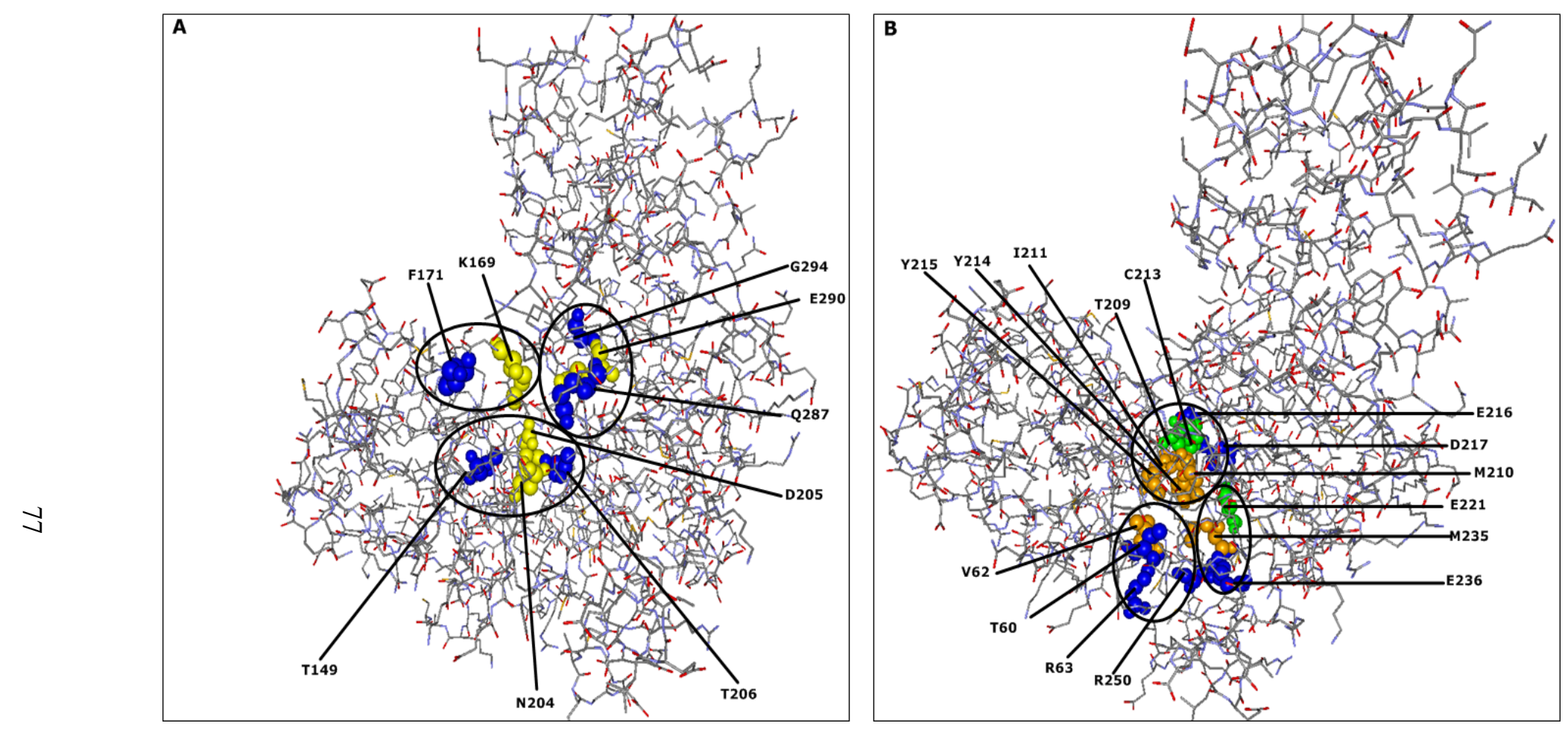

Figure 6.6: CMF-significant residue positions are in contact with glucose binding site and allosteric site in human GCK protein (PDB-Entry 1V4S). (A) Yellow spheres show the structural positions of glucose binding sites (active sites). Blue spheres correspond to localization of CMF-significant adjacent residue positions which are in contact with these active sites. (B) Orange spheres denote the allosteric sites. Blue spheres correspond to localization of just significant adjacent residue positions and green spheres indicate the significant residue positions which are already described as nsSNP positions and in contact with these allosteric sites. Additionally, the significant position R63 is allosteric site by itself and it is also in contact with another allosteric site. Circles indicate clusters of glucose binding sites (A), allosteric sites (B), and their significant adjacent sites. 
Besides this, there are further $30 \mathrm{CMF}$-significant essential sites which are nearby nsSNPs or strictly conserved residue positions (see Table 6.4). Altogether, we showed the functionality of 57 positions out of 72 CMF-significant residue sites via different resources [34-40].

\begin{tabular}{|c|c|c|}
\hline CMF-significant essential sites & Nearby nsSNPs or strictly conserved sites & References \\
\hline$M 34$ & $36^{\mathbf{s}}$ & $\mid 39$ \\
\hline$T 65$ & $66^{c}$ & \\
\hline$E 67$ & $66^{\mathbf{c}}, 68^{\mathbf{c}}$ & - \\
\hline$T 82$ & $81^{\mathrm{c}}$ & - \\
\hline$N 83$ & $81^{\mathbf{c}}, 108^{\mathbf{s}}, 110^{\mathrm{s}}$ & $\mid 39$ \\
\hline$H 105$ & $106^{\mathrm{s}}$ & $|\overline{34}|$ \\
\hline$C 129$ & $131^{\mathrm{s}}, 132^{\mathrm{s}}$ & 3439 \\
\hline$F 133$ & $131^{\mathrm{s}}, 132^{\mathrm{s}}$ & |34 39 \\
\hline$F 148$ & $147^{\mathbf{c}}, 150^{\mathbf{c}, \mathbf{s}}$ & $|\overline{39}|$ \\
\hline$F 152$ & $150^{\mathbf{c}, \mathbf{s}}, 151^{\mathrm{c}}$ & $|\overline{39}|$ \\
\hline$H 156$ & $162^{\mathrm{s}}$ & 34 \\
\hline$N 180$ & $162^{\mathrm{s}}, 182^{\mathrm{s}}$ & 34 \\
\hline$F 260$ & $257^{\mathrm{s}}, 258^{\mathrm{c}}, 259^{\mathrm{s}}, 261^{\mathrm{s}}$ & 39 \\
\hline$D 262$ & $259^{\mathrm{s}}, 261^{\mathrm{s}}, 264^{\mathrm{s}}$ & $\mid 92$ \\
\hline$L 266$ & $261^{\mathrm{s}}, 264^{\mathrm{s}}, 265^{\mathrm{s}}$ & $|\overline{34}| \overline{39} \mid 92$ \\
\hline D267 & $264^{\mathrm{s}}, 265^{\mathrm{s}}$ & $|\overline{34}| \overline{92} \mid$ \\
\hline$L 271$ & $274^{\mathrm{c}}$ & \\
\hline$S 281$ & $278^{\mathrm{c}}, 279^{\mathrm{s}}$ & 39 \\
\hline$Q 286$ & $259^{s}$ & $|\overline{39}|$ \\
\hline E331 & $299^{\mathbf{c}, \mathbf{s}}$ & 39 \\
\hline$T 332$ & $295^{\mathbf{c}}, 299^{\mathbf{c}, \mathbf{s}}$ & 39 \\
\hline$R 333$ & $336^{\mathrm{s}}$ & 39 \\
\hline$Q 337$ & $336^{\mathrm{s}}$ & $|\overline{39}|$ \\
\hline E339 & $336^{\mathrm{s}}$ & $|\overline{39}|$ \\
\hline$N 391$ & $392^{\mathrm{s}}$ & |34 39 \\
\hline$S 411$ & $227^{\mathbf{c}, \mathbf{s}}, 410^{\mathbf{c}}, 414^{\mathbf{s}}$ & |39] \\
\hline$S 418$ & $416^{\mathrm{s}}$ & 35 \\
\hline$F 419$ & $416^{\mathrm{s}}$ & $|\overline{35}|$ \\
\hline$E 442$ & $444^{c}$ & \\
\hline$E 443$ & $444^{\mathrm{c}}, 445^{\mathrm{c}}$ & . \\
\hline
\end{tabular}

Table 6.4: CMF-significant essential sites in human GCK protein, which are nearby either nsSNPs or strictly conserved sites. ${ }^{\mathbf{s}}$ : non-synonymous snp site, ${ }^{\mathbf{c}}$ : strictly conserved site.

While we are able to establish the large number of CMF-significant sites as structurally or functionally important in human GCK protein, 15 CMF-significant sites do not overlap with essential sites. Their importance in the GCK protein and the reason of high connectivity degree of these unconfirmed significant sites has not been determined yet. 


\subsubsection{A comparison between $\mathbb{U}$-metric and $\mathbb{U}_{D(\alpha)}$-metric}

Similarities in physical or biochemical properties of amino acids are likely to be crucial for the detection of functionally or structurally important positions of a protein. In contrast to the $\mathbb{U}$-metric, which is a normalized mutual information that uses only the frequencies of occurrences of amino acids in the MSA columns, the novel $\mathbb{U}_{D(\alpha)}$-metric includes dissimilarities according to the BLOSUM62 matrix when calculating normalized mutual information. As a result, the positions which have undergone dissimilar compensatory mutations are upscaled.

Having applied the $\mathbb{U}$-metric as well as the $\mathbb{U}_{D(\alpha)}$-metric to human EGFR and GCK proteins, the $\mathbb{U}_{D(\alpha)}$-metric has shown better sensitivity and specificity. However, only when we use the both metrics together, the sensitivity is significantly increased, whereas the specificity is only moderately decreased. The details are presented in Table 6.5 .

\begin{tabular}{|l|c|c|}
\hline & Sensitivity & Specificity \\
\hline $\mathbb{U}$-significance & $9.7 \%$ & $91.5 \%$ \\
$\mathbb{U}_{D(\alpha)}$-significance & $12.4 \%$ & $97.2 \%$ \\
CMF-significance & $22.1 \%$ & $88.7 \%$ \\
\hline
\end{tabular}

Table 6.5: Comparison between $\mathbb{U}$-metric and $\mathbb{U}_{D(\alpha)}$-metric

It is important to note that the two metrics complement each other, thus we propose to use them together. 


\subsection{Applying the $\mathbb{Q}_{\text {ent }}$ and the $\mathbb{Q}_{\text {sep-metric for the human EGFR }}$ and GCK proteins}

In Section 4.2, we pointed out that column pair metric distributions on the interval $[0,1]$ can be modeled based on distributions of background signals $F_{0}$, unrelated pair signals $G_{1}$, and significant pair signals $G_{2}$, where $F_{0}$ is a $\beta$-distribution. Let $\mathbb{Q}$ be one of the two new metrics introduced in Section 5.2. The $p$-values $\left(1-F_{0}\left(\mathbb{Q}_{i}\right)\right), i=1,2, \ldots$ are uniformly distributed over $[0,1]$, if $\mathbb{Q}$-values are $F_{0}$-distributed. In contrast, $p$-values tend to zero or one, if $\mathbb{Q}$-values are $G_{2}$-distributed or $G_{1}$-distributed, respectively (see Figure 6.7). By slightly generalizing the Storey-Tibshirani procedure devised for multiple testing problems $[27,28,75]$ to quantify the error made in terms of false discovery rate (FDR), we have developed in our MSA-specific statistical model which successfully separates significant signals of column pairs from background signals and unrelated pair signals. In order to determine significant $\mathbb{Q}$-values of column pairs, we also apply that model in this section. After that, we utilized the connectivity degree technique (see Section 4.2.2) in order to characterize the significance of individual residue sites.

Let $M$ be an MSA, where the protein of interest is represented by $M$ 's first row. The definition of a $(\mathbb{Q}, M)$-significant site pair $(i, j)$ of the protein is completely analogous to the corresponding notion presented in the previous Section 6.2. A residue site of the protein is defined to be QCMF-significant 9 with respect to the MSA $M$, if it is $\left(\mathbb{Q}_{\text {ent }}, M\right)$-significant or $\left(\mathbb{Q}_{\text {sep }}, M\right)$-significant and its connectivity degree cut-off exceeds the 90 -th percentile in $\left(\mathbb{Q}_{\text {ent }}, M\right)$-significant or $\left(\mathbb{Q}_{\text {sep }}, M\right)$-significant pairs.

In order to compare the performance and functionality of our quantum information theory based method with our classical information theory based method, we tested again the same human proteins using their related MSAs for the evaluation of QCMF-significant residue sites. Likewise, we define here a QCMF-significant residue site as "functionally or structurally important" if it corresponds to one of the essential sites.

\subsubsection{Position analysis of the Human EGFR protein}

Using the MSA-specific statistical model with a false discovery rate (FDR) of $1 \%$ for both

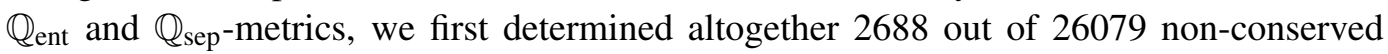
column pairs as significant in corresponding MSA of human EGFR protein. 631 of these significant pairs are detected by the $\mathbb{Q}_{\text {ent }}$-metric and 2149 pairs are observed by the $\mathbb{Q}_{\text {sep}^{-}}$ metric. Only 92 significant column pairs were detected by both metrics. After that, utilizing the connectivity degree technique, we predicted a total of 33 residue sites in the corresponding sequence of the human EGFR protein as QCMF-significant (see Table 6.6). 12 of them

\footnotetext{
${ }^{9}$ We defined the predicted significant sites together as QCMF-significant due to our corresponding publication "Quantum Coupled $\underline{\text { Mutation }}$ Finder" [5].
} 


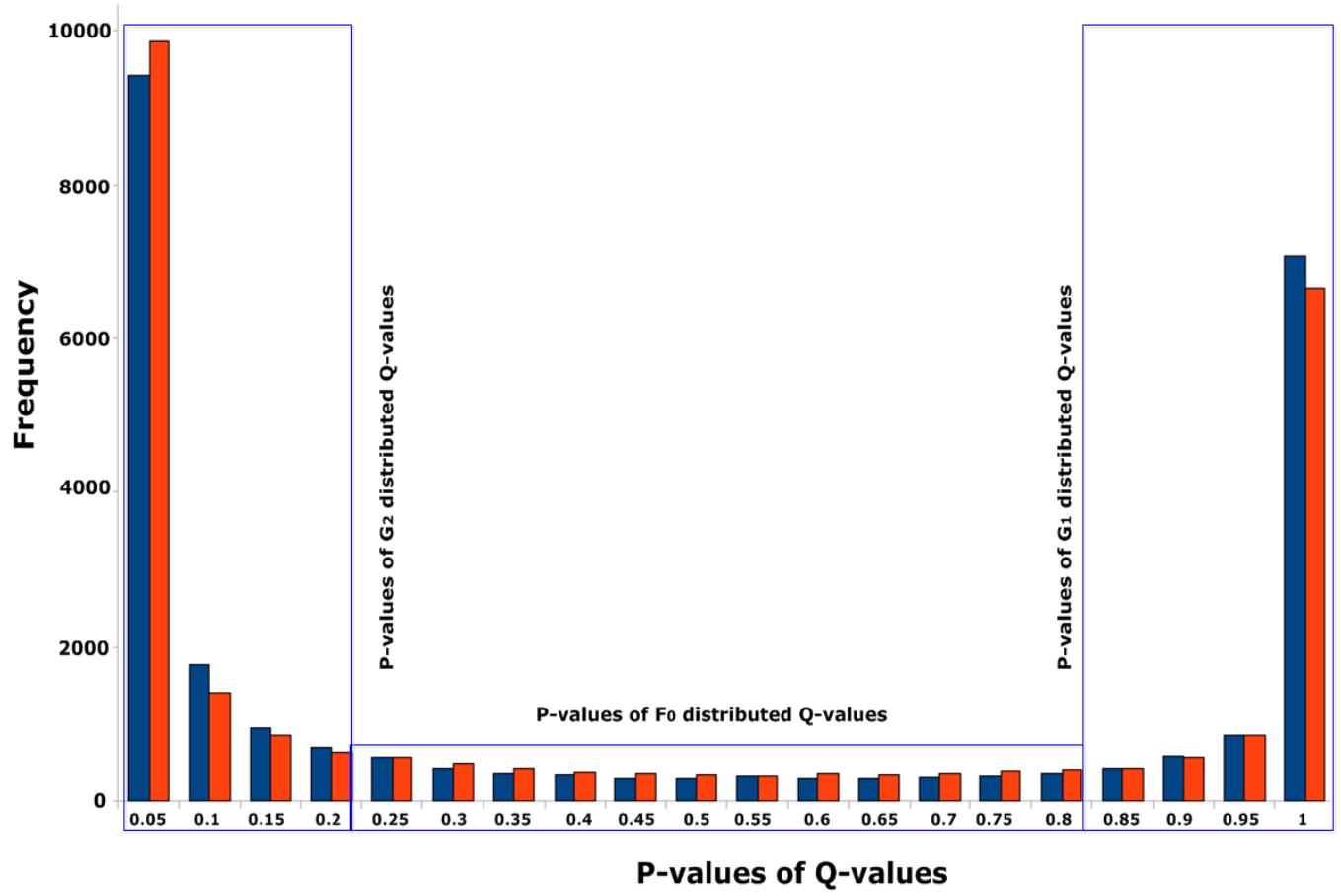

Figure 6.7: p-value distributions of $\mathbb{Q}_{\text {ent }}$ and $\mathbb{Q}_{\text {sep-values for human EGFR protein }}$ (PDB-Entry 2J6M). The blue bars illustrate the $p$-value distribution of the $\mathbb{Q}_{\text {ent-values }}$ and red bars display the $p$-value distribution of the $\mathbb{Q}_{\text {sep-values. }}$ -

are only $\mathbb{Q}_{\text {ent }}$-significant and 18 residue sites are $\mathbb{Q}_{\text {sep-significant, the remaining } 3 \text { residue }}$ sites (A839,A882 and V902) are determined both $\mathbb{Q}_{\text {ent }}$-significant and $\mathbb{Q}_{\text {sep-significant. }}$

10 of the QCMF-significant residue sites are in contact with either catalytic residues or critical active site regions for gefitinib binding site in wild type EGFR kinase [30, 33, 58] (see Figure 6.8 and Figure 6.9). Among these sites, the A839 and R841 have been verified as catalytic residue sites through Catalytic Site Atlas [58], the T854 is a gefitinib binding site by itself and the residue sites V845 and A859 are also in contact with nsSNP positions K846, T847 and K860 in human EGFR protein. Moreover, two out of all 33 significant sites are related to disease associated nsSNP positions and their structural localization are illustrated in Figure 6.8 . 


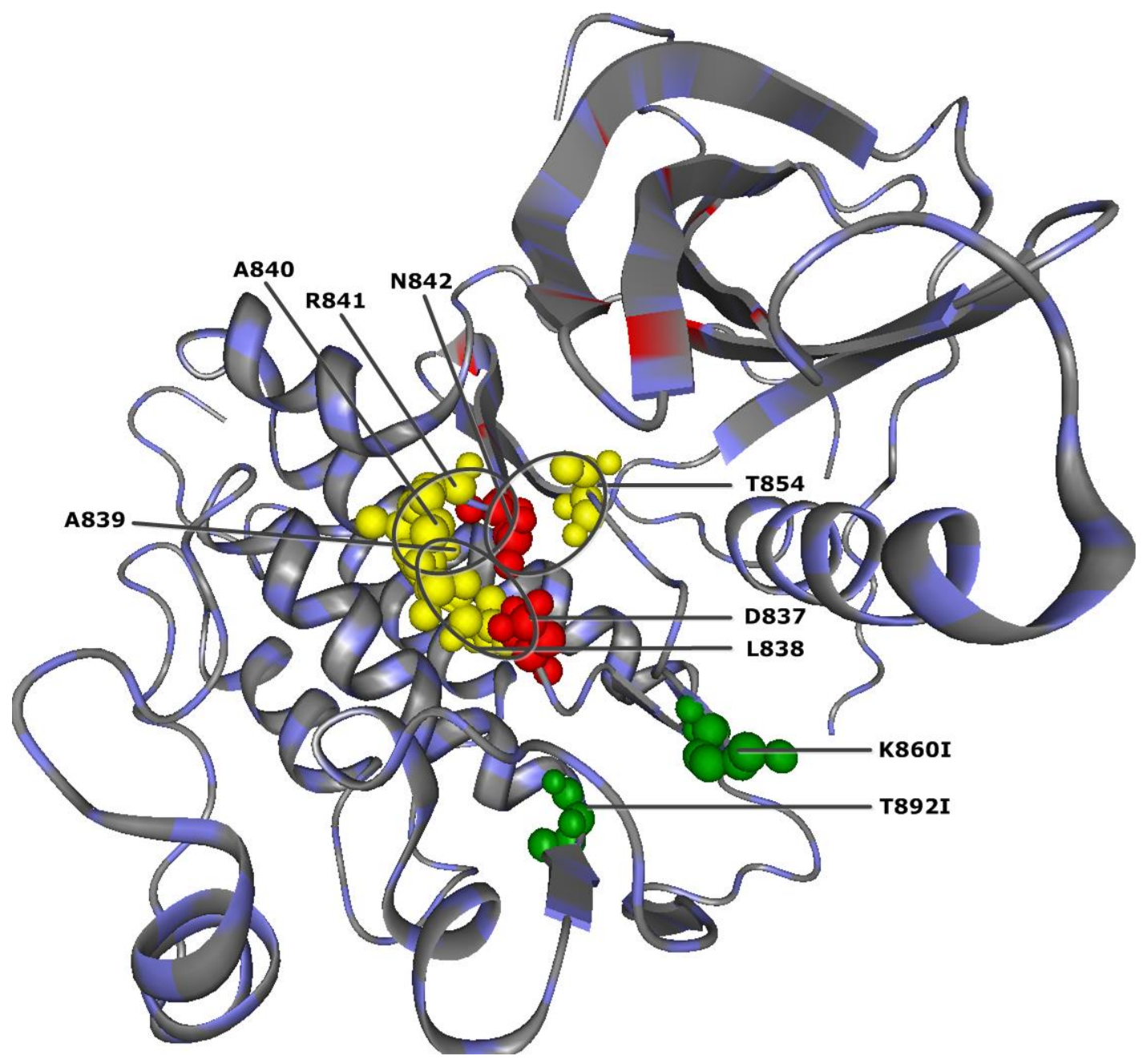

Figure 6.8: QCMF-significant residue positions are in contact with catalytic residues in human EGFR protein (PDB-Entry 2J6M). Red spheres denote positions of the catalytic residues. Yellow spheres show the localization of QCMF-significant adjacent residue positions which are in contact with these catalytic residues. Moreover, the QCMF-significant sites A839 and R841 are also catalytic residues by themselves. Green spheres show the structural localization of QCMF-significant nsSNP positions in the EGFR protein. Circles indicate clusters of catalytic residue sites and their significant adjacent sites. 


\begin{tabular}{|c|c|c|c|c|c|c|c|}
\hline Residue & Position & Connectivity Degree & Detected by & Residue & Position & Connectivity Degree & Detected by \\
\hline G & 729 & 62 & $\mathbb{Q}_{\text {sep}}$-metric & $\mathrm{L}$ & 844 & 62 & $\mathbb{Q}_{\text {sep-metric }}$ \\
\hline $\mathrm{T}$ & 751 & 18 & Qent-metric & V & 845 & 97 & $\mathbb{Q}$ ent-metric \\
\hline $\mathrm{N}$ & 771 & 57 & $\mathbb{Q}_{\text {sep-metric }}$ & I & 853 & 21 & $\mathbb{Q}_{\text {ent-metric }}$ \\
\hline G & 779 & 70 & $\mathbb{Q}_{\text {sep-metric }}$ & $\mathrm{T}$ & 854 & 24 & $\mathbb{Q}_{\text {ent-metric }}$ \\
\hline Q & 791 & 77 & $\mathbb{Q}_{\text {sep-metric }}$ & A & 859 & 24 & $\mathbb{Q}$ ent-metric \\
\hline I & 792 & 22 & $\mathbb{Q}_{\text {ent}}$-metric & K & 860 & 78 & $\mathbb{Q}_{\text {sep-metric }}$ \\
\hline Q & 820 & 69 & $\mathbb{Q}_{\text {sep-metric }}$ & A & $882^{*}$ & 26 & $\mathbb{Q}_{\text {ent-metric }}$ \\
\hline A & 822 & 35 & $\mathbb{Q}$ ent-metric & A & $882^{*}$ & 54 & $\mathbb{Q}_{\text {sep-metric }}$ \\
\hline G & 824 & 89 & $\mathbb{Q}_{\text {sep-metric }}$ & $\mathrm{Y}$ & 891 & 113 & $\mathbb{Q}_{\text {sep-metric }}$ \\
\hline M & 825 & 72 & $\mathbb{Q}_{\text {sep-metric }}$ & $\mathrm{T}$ & 892 & 45 & Qent-metric \\
\hline $\mathrm{Y}$ & 827 & 119 & $\mathbb{Q}_{\text {sep-metric }}$ & $\mathrm{Y}$ & 900 & 132 & $\mathbb{Q}_{\text {sep-metric }}$ \\
\hline $\mathrm{L}$ & 828 & 52 & $\mathbb{Q}_{\text {sep-metric }}$ & V & $902^{*}$ & 74 & $\mathbb{Q}$ ent-metric \\
\hline V & 834 & 54 & $\mathbb{Q}$ ent-metric & V & $902^{*}$ & 79 & $\mathbb{Q}_{\text {sep-metric }}$ \\
\hline $\mathrm{L}$ & 838 & 52 & $\mathbb{Q}_{\text {sep-metric }}$ & $\mathrm{T}$ & 909 & 42 & $\mathbb{Q}_{\text {ent-metric }}$ \\
\hline A & $839^{*}$ & 43 & $\mathbb{Q}_{\text {ent}}$-metric & G & 911 & 66 & $\mathbb{Q}_{\text {sep-metric }}$ \\
\hline A & $839^{*}$ & 55 & $\mathbb{Q}_{\text {sep-metric }}$ & $\mathrm{L}$ & 927 & 24 & Qent-metric \\
\hline A & 840 & 128 & Qent-metric & G & 930 & 71 & $\mathbb{Q}_{\text {sep-metric }}$ \\
\hline $\mathrm{R}$ & 841 & 68 & $\mathbb{Q}_{\text {sep-metric }}$ & $\mathrm{Y}$ & 944 & 51 & $\mathbb{Q}_{\text {sep-metric }}$ \\
\hline
\end{tabular}

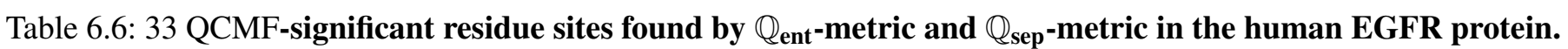
${ }^{*}$ denotes the residue sites are detected by both metrics $\mathbb{Q}_{\text {ent }}$ and $\mathbb{Q}_{\text {sep }}$ as significant. 


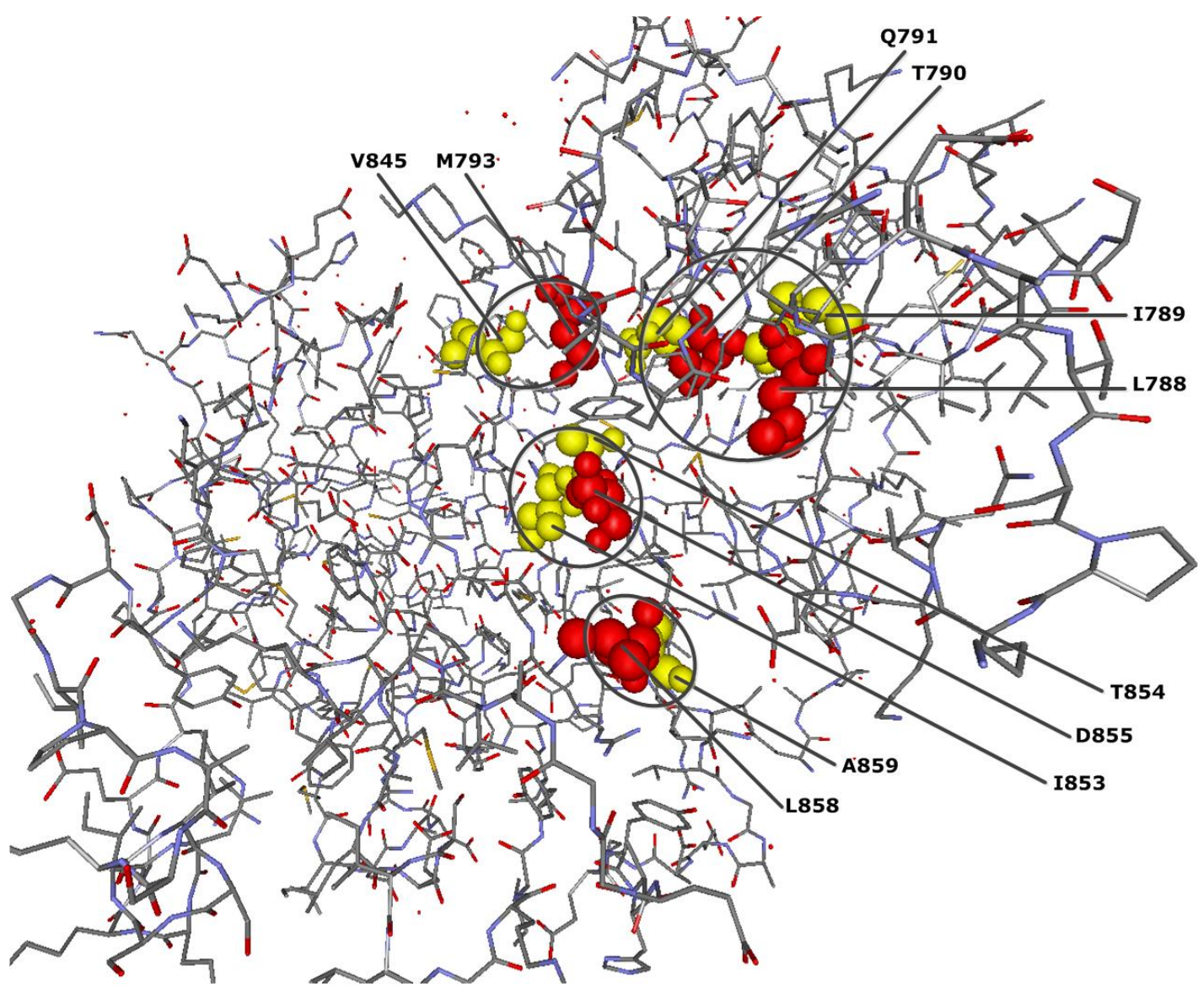

Figure 6.9: QCMF-significant residue positions are in contact with gefitinib binding sites in human EGFR protein (PDB-Entry 2J6M). Red spheres show the structural localization of the gefitinib binding sites in the wild type kinase. Yellow spheres show significant adjacent residue positions which are in contact with these binding sites. Moreover, the QCMF-significant site T854 is also binding site by itself and interacts with gefitinib binding sites D855. Circles indicate clusters of gefitinib binding sites and their significant adjacent sites.

Additionally, 13 of out of all QCMF-significant sites are defined as essential sites since they are either nearby strictly conserved residues or nsSNPs (see Table 6.7). 
6.3 Applying the $\mathbb{Q}_{\text {ent }}$ and the $\mathbb{Q}_{\text {sep-metric for the human EGFR and GCK proteins }}$

\begin{tabular}{|c|c|c|}
\hline QCMF-significant essential sites & Nearby nsSNPs or strictly conserved sites & References \\
\hline$N 771$ & $773^{\mathrm{s}}$ & $|40|$ \\
\hline$G 824$ & $773^{\mathrm{s}}$ & |40] \\
\hline$Y 827$ & $829^{\mathbf{s}}$ & {$[\overline{40}]$} \\
\hline$L 828$ & $829^{\mathrm{s}}$ & 40 \\
\hline$V 834$ & $835^{\mathbf{c}}, 836^{\mathbf{s}}, 860^{\mathbf{s}}$ & {$[\overline{40} 92$} \\
\hline$Y 891$ & $892^{\mathrm{s}}, 895^{\mathrm{c}}$ & {$[\overline{40}$} \\
\hline A822 & $861^{\mathrm{s}}$ & {$\left[\begin{array}{|l|l|}29 & 39\end{array}\right.$} \\
\hline$V 844$ & $796^{\mathbf{c}}, 798^{\mathbf{c}}, 852^{\mathbf{c}}$ & \\
\hline$A 882$ & $884^{\mathbf{c}}, 895^{\mathbf{c}}, 898^{\mathbf{c}}$ & - \\
\hline$Y 900$ & $898^{\mathbf{c}}, 901^{\mathbf{c}}$ & - \\
\hline$V 902$ & $880^{\mathbf{c}}, 901^{\mathbf{c}}$ & - \\
\hline T909 & $906^{\mathbf{c}}, 936^{\mathbf{c}}$ & - \\
\hline$G 911$ & $906^{\mathbf{c}}$ & - \\
\hline
\end{tabular}

Table 6.7: QCMF-significant essential sites in human EGFR protein, which are nearby either nsSNPs or strictly conserved sites. ${ }^{\mathbf{s}}$ : non-synonymous snp site, ${ }^{\mathbf{c}}$ : strictly conserved site.

According to the essential sites of human EGFR protein, we have shown altogether the structural or functional importance of $25 \mathrm{QCMF}-$ significant sites. The remaining 8 significant residue sites (G729, T851, G779, Q820, M825, L927, G930, Y944) do not fall into essential sites and the reason for their significance and their importance in the EGFR protein is currently unclear.

Finally, we compared the new QCMF-significant residue sites for human EGFR protein with our previous CMF-significant residue sites. The new significant residue sites Q791, Q820, G824, K860, Y891, T892, Y900, T909 overlap with CMF-significant residue sites. Interestingly, one of the unconfirmed residue sites (Q820) has been predicted as both QCMFsignificant and CMF-significant.

\subsubsection{Position analysis of the the Human GCK protein}

Like human EGFR protein, applying the MSA-specific statistical model with a FDR of $1 \%$ for both $\mathbb{Q}_{\text {ent }}$ and $\mathbb{Q}_{\text {sep}}$-metrics we identified a total of 9853 out of 69645 non-conserved column pairs as significant in human GCK protein. 6070 of them are $\left(\mathbb{Q}_{\text {ent }}, M\right)$-significant and 4232 are detected as $\left(\mathbb{Q}_{\text {sep }}, M\right)$-significant. Only 449 column pairs are determined as significant with respect to both metrics. Thereupon using the connectivity degree technique, we determined altogether 64 residue sites in human GCK protein as QCMF-significant (see Table 6.8). 30 of them are observed as $\mathbb{Q}_{\text {ent }}$-significant and 30 significant residue sites are detected as $\mathbb{Q}_{\text {sep-significant. Only four residue sites (T82, G223, V253, G407) are defined }}$ as significant based on both metrics. 


\begin{tabular}{|c|c|c|c|c|c|c|c|}
\hline Residue & Position & Connectivity Degree & Detected by & Residue & Position & Connectivity Degree & Detected by \\
\hline $\mathrm{M}$ & 37 & 75 & $\mathbb{Q}_{\text {sep-metric }}$ & $\bar{G}$ & $223^{*}$ & 97 & $\mathbb{Q}_{\text {sep-metric }}$ \\
\hline $\mathrm{T}$ & 60 & 177 & $\mathbb{Q}$ ent-metric & G & $223^{*}$ & 63 & $\mathbb{Q}_{\text {ent}}$-metric \\
\hline Y & 61 & 87 & $\mathbb{Q}_{\text {sep-metric }}$ & $\mathrm{T}$ & 228 & 310 & $\mathbb{Q}$ ent-metric \\
\hline V & 62 & 65 & Qent-metric & A & 232 & 184 & $\mathbb{Q}_{\text {ent}}$-metric \\
\hline$S$ & 76 & 297 & $\mathbb{Q}_{\text {ent}}$-metric & $\mathrm{C}$ & 233 & 241 & $\mathbb{Q}_{\text {ent }}$-metric \\
\hline $\mathrm{L}$ & 79 & 70 & $\mathbb{Q}_{\text {sep-metric }}$ & E & 236 & 153 & $\mathbb{Q}_{\text {sep-metric }}$ \\
\hline $\mathrm{T}$ & $82^{*}$ & 95 & $\mathbb{Q}_{\text {sep-metric }}$ & V & $253^{*}$ & 89 & $\mathbb{Q}_{\text {sep-metric }}$ \\
\hline $\mathrm{T}$ & $82^{*}$ & 318 & $\mathbb{Q}_{\text {ent}}$-metric & V & $253^{*}$ & 64 & $\mathbb{Q}_{\text {ent }}$-metric \\
\hline $\mathrm{N}$ & 83 & 108 & $\mathbb{Q}_{\text {sep-metric }}$ & $\mathrm{F}$ & 260 & 84 & $\mathbb{Q}_{\text {sep-metric }}$ \\
\hline V & 86 & 144 & $\mathbb{Q}_{\text {ent-metric }}$ & $\mathrm{L}$ & 271 & 171 & $\mathbb{Q}_{\text {ent }}$-metric \\
\hline V & 89 & 67 & $\mathbb{Q}_{\text {ent}}$-metric & V & 277 & 107 & $\mathbb{Q}_{\text {ent }}$-metric \\
\hline $\mathrm{F}$ & 123 & 161 & $\mathbb{Q}_{\text {sep-metric }}$ & $\mathrm{S}$ & 281 & 99 & $\mathbb{Q}_{\text {sep-metric }}$ \\
\hline $\mathrm{S}$ & 127 & 296 & $\mathbb{Q}_{\text {ent }}$-metric & $\mathrm{N}$ & 283 & 91 & $\mathbb{Q}_{\text {sep-metric }}$ \\
\hline $\mathrm{F}$ & 148 & 101 & $\mathbb{Q}_{\text {sep-metric }}$ & Q & 287 & 96 & $\mathbb{Q}_{\text {sen-metric }}$ \\
\hline $\mathrm{T}$ & 149 & 318 & $\mathbb{Q}$ ent-metric & G & 294 & 62 & $\mathbb{Q}$ ent-metric \\
\hline $\mathrm{F}$ & 152 & 207 & $\mathbb{Q}_{\text {sep-metric }}$ & Y & 297 & 178 & $\mathbb{Q}_{\text {sep-metric }}$ \\
\hline $\mathrm{P}$ & 153 & 95 & $\mathbb{Q}_{\text {sep-metric }}$ & M & 298 & 81 & $\mathbb{Q}_{\text {sep-metric }}$ \\
\hline $\mathrm{H}$ & 156 & 68 & $\mathbb{Q}_{\text {sep-metric }}$ & $\mathrm{E}$ & 300 & 82 & $\mathbb{Q}_{\text {sep-metric }}$ \\
\hline G & 162 & 95 & $\mathbb{Q}_{\text {sep-metric }}$ & $\mathrm{T}$ & 332 & 89 & $\mathbb{Q}_{\text {ent-metric }}$ \\
\hline G & 170 & 82 & $\mathbb{Q}_{\text {sep-metric }}$ & V & 374 & 182 & $\mathbb{Q}$ ent-metric \\
\hline $\mathrm{F}$ & 171 & 144 & $\mathbb{Q}_{\text {sep-metric }}$ & A & 378 & 302 & $\mathbb{Q}$ ent-metric \\
\hline G & 175 & 90 & $\mathbb{Q}_{\text {sep-metric }}$ & A & 379 & 292 & $\mathbb{Q}$ ent-metric \\
\hline A & 176 & 63 & $\mathbb{Q}_{\text {ent}}$-metric & S & 383 & 198 & $\mathbb{Q}_{\text {ent}}$-metric \\
\hline G & 178 & 125 & $\mathbb{Q}_{\text {sep-metric }}$ & A & 384 & 55 & $\mathbb{Q}_{\text {ent}}$-metric \\
\hline $\mathrm{N}$ & 180 & 76 & $\mathbb{Q}_{\text {sep-metric }}$ & A & 387 & 59 & $\mathbb{Q}_{\text {ent }}$-metric \\
\hline $\mathrm{L}$ & 185 & 96 & $\mathbb{Q}_{\text {sep-metric }}$ & G & 388 & 70 & $\mathbb{Q}_{\text {ent }}$-metric \\
\hline A & 201 & 308 & $\mathbb{Q}_{\text {ent-metric }}$ & G & $407^{*}$ & 75 & $\mathbb{Q}_{\text {sep-metric }}$ \\
\hline M & 202 & 105 & $\mathbb{Q}_{\text {ent-metric }}$ & G & $407^{*}$ & 143 & $\mathbb{Q}$ ent-metric \\
\hline $\mathrm{T}$ & 206 & 300 & $\mathbb{Q}_{\text {ent-metric }}$ & V & 412 & 218 & $\mathbb{Q}_{\text {ent }}$-metric \\
\hline V & 207 & 303 & $\mathbb{Q}_{\text {ent-metric }}$ & $\mathrm{F}$ & 419 & 110 & $\mathbb{Q}_{\text {sep-metric }}$ \\
\hline A & 208 & 145 & $\mathbb{Q}_{s e p}$-metric & $\mathrm{E}$ & 443 & 110 & $\mathbb{Q}_{\text {sep-metric }}$ \\
\hline $\mathrm{T}$ & 209 & 319 & $\mathbb{Q}$ ent-metric & G & 446 & 86 & $\mathbb{Q}_{\text {sep-metric }}$ \\
\hline M & 210 & 85 & $\mathbb{Q}_{s e p}$-metric & $\mathrm{L}$ & 451 & 63 & $\mathbb{Q}_{\text {ent }}$-metric \\
\hline $\mathrm{Y}$ & 215 & 104 & $\mathbb{Q}_{\text {sep-metric }}$ & S & 453 & 317 & $\mathbb{Q}_{\text {ent}}$-metric \\
\hline
\end{tabular}

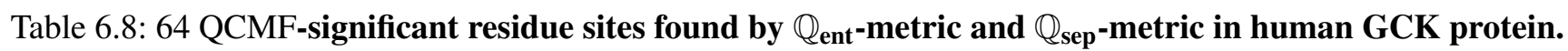

* denotes the residue sites are detected by both metrics $\mathbb{Q}_{\text {ent }}$ and $\mathbb{Q}_{\text {sep }}$ as significant. 
13 out of all QCMF-significant residue sites are in contact with allosteric sites V62, R63, M210, I211, Y214, Y215, M235, V452, V455 and A456 in human GCK protein. Among these significant sites, the $V 62, M 210, Y 215$ are allosteric sites by themselves [37] and the T209M, G223S and S453del are related to disease associated nsSNP positions. In addition, there are further five QCMF-significant sites F123L, G162D, G175R, T228M and E300K,Q that have been verified as nsSNP positions through annotation databases and previous experimental studies [34, 36, 38,39,93]. The structural localization of these 18 QCMF-significant residue sites (contact sites and nsSNPs positions) are illustrated in Figure 6.10.

Additionally, eight QCMF-significant sites T149, G170, F171, T206, V207, A208, Q287 and G294 interact with glucose binding sites (active sites) T168, K169, D204, D205 and E290 in human GCK protein [37] (see Figure 6.11) where V207 and A208 are also in contact with allosteric sites M210 and I211.

Moreover, we have observed that 38 QCMF-significant sites are further included in essential sites since they are near nsSNPs or strictly conserved residues in human GCK protein (see Table 6.9.

\begin{tabular}{|c|c|c|}
\hline QCMF-significant essential sites & Nearby nsSNPs or strictly conserved sites & References \\
\hline M37 & $36^{\mathrm{s}}, 39^{\mathrm{s}}, 40^{\mathrm{s}}$ & \begin{tabular}{|l|l|l|}
34 & 35 & 39 \\
\end{tabular} \\
\hline$S 76$ & $147^{\mathrm{c}}$ & \\
\hline$L 79$ & $78^{\mathrm{c}}, 80^{\mathrm{c}}, 150^{\mathrm{c}}$ & - \\
\hline$T 82$ & $81^{\mathrm{c}}$ & \\
\hline N83 & $81^{\mathbf{c}}, 108^{\mathrm{s}}, 110^{\mathrm{s}}$ & [39 93 \\
\hline$V 86$ & $85^{\mathbf{c}}, 106^{\mathrm{s}}$ & [34] \\
\hline$S 127$ & $130^{\mathrm{s}}$ & {$[\overline{36}]$} \\
\hline$F 148$ & $147^{\mathbf{c}}, 150^{\mathbf{c}, \mathbf{s}}$ & $34,35,39,93$ \\
\hline$F 152$ & $150^{\mathbf{c}, \mathbf{s}}, 151^{\mathbf{c}}$ & [35, $\overline{39}, \overline{93}$ \\
\hline$P 153$ & $154^{\mathrm{s}}$ & [35] \\
\hline$H 156$ & $154^{\mathrm{s}}$ & {$[\overline{35}$} \\
\hline A176 & $119^{\mathrm{s}}, 175^{\mathrm{s}}$ & {$[\overline{39}]$} \\
\hline G178 & $164^{\mathrm{c}}$ & \\
\hline$N 180$ & $162^{\mathrm{s}}, 182^{\mathrm{s}}$ & [34,35,39], \\
\hline$L 185$ & $182^{\mathrm{s}}, 188^{\mathrm{s}}$ & [35. 39.93 \\
\hline A201 & $147^{\mathrm{c}}, 453^{\mathrm{c}}$ & \\
\hline M202 & $147^{\mathrm{c}}, 203^{\mathrm{s}}$ & \\
\hline A232 & $223^{\mathrm{s}}, 231^{\mathrm{c}}$ & [35, 36, 93 \\
\hline$C 233$ & $223^{\mathrm{s}}, 234^{\mathrm{c}}, 235^{\mathrm{c}}$ & [35 $\mid \overline{36}, \overline{93}]$ \\
\hline$V 253$ & $234^{\mathrm{c}}, 254^{\mathrm{c}}$ & \\
\hline$F 260$ & $257^{\mathrm{s}}, 258^{\mathrm{c}}, 259^{\mathrm{s}}, 261^{\mathrm{s}}$ & {$[35,39$} \\
\hline$L 271$ & $274^{\mathrm{c}}$ & \\
\hline V277 & $274^{\mathrm{c}}, 278^{\mathrm{c}}, 279^{\mathrm{s}}$ & [39] \\
\hline$S 281$ & $278^{c}, 279^{s}$ & [39] \\
\hline Y297 & $291^{\mathbf{c}}, 295^{\mathbf{c}}, 299^{\mathbf{c}}, 300^{\mathbf{s}}$ & {$[\overline{39}]$} \\
\hline M298 & $295^{\mathbf{c}}, 299^{\mathbf{c}}, 300^{\mathrm{s}}$ & {$[\overline{39]}$} \\
\hline$T 332$ & $295^{\mathbf{c}}, 299^{\mathbf{c}}$ & \\
\hline
\end{tabular}


Table 6.9 - continued from previous page

\begin{tabular}{|c|l|c|}
\hline QCMF-significant essential sites & Nearby nsSNPs or strictly conserved sites & References \\
\hline$V 374$ & $377^{\mathbf{c}}$ & - \\
$A 378$ & $377^{\mathbf{c}}, 382^{\mathbf{s}}$ & $\mid 39$ \\
$A 379$ & $377^{\mathbf{c}}, 382^{\mathbf{s}}$ & $\mid 39$ \\
$S 383$ & $382^{\mathbf{s}}, 385^{\mathbf{s}}$ & $\mid 39$ \\
$A 384$ & $382^{\mathbf{s}}, 385^{\mathbf{s}}$ & $\mid 39$ \\
$A 387$ & $385^{\mathbf{s}}$ & $\mid 39$ \\
$S 388$ & $385^{\mathbf{s}}, 392^{\mathbf{s}}$ & $\mid 39$ \\
$V 412$ & $226^{\mathbf{s}}, 227^{\mathbf{c}}, 410^{\mathbf{c}}, 414^{\mathbf{s}}, 416^{\mathbf{s}}$ & $\mid 35$ \\
$F 419$ & $416^{\mathbf{s}}$ & $\mid 35$ \\
\hline$E 443$ & $444^{\mathbf{c}}, 445^{\mathbf{c}}, 447^{\mathbf{s}}$ & \\
\hline
\end{tabular}

Table 6.9: QCMF-significant essential sites in human GCK protein, which are nearby either nsSNPs or strictly conserved sites. ${ }^{\mathbf{s}}$ : non-synonymous snp site, ${ }^{\mathbf{c}}$ : strictly conserved site.

In total, we have demonstrated here that according to the essential sites, 62 out of 64 QCMFsignificant residue sites are functionally or structurally important for human GCK protein. The remaining two significant residue sites V89, N283 do not overlap with essential sites and the reason for their significance and their role in the GCK protein has not been determined yet.

Lastly, we compared the new QCMF-significant residue sites with the previous CMFsignificant sites. The unconfirmed residue site N283 and further 23 significant sites (T60, T82, N83, F123, F148, T149, F152, H156, F171, N180, T206, T209, T228, E236, G260, L271, S281, Q287, G294, E300, T332, F419 and E443) were also determined by the previous method as significant.

\subsection{A Comparison between CMF-significant sites and QCMF-significant sites}

To further investigate the performance of our methods, we made a statistical comparison between classical information theory based CMF and quantum information theory based QCMF. The CMF method mainly focuses on significant BLOSUM62-dissimilar amino acid signals as a model of compensatory mutations and integrates them in the calculation of metrics. As a consequence of only taking into account dissimilar amino acid signals, an important part of its significant sites are verified as disease associated nsSNP positions and just a small part of them were located at or near other essential sites in both proteins. In contrast, considerung simultinously BLOSUM62-similar and dissimilar amino acid signals, the result of our new quantum information theory based method is more sensible to catalytic sites, allosteric sites and binding sites. 


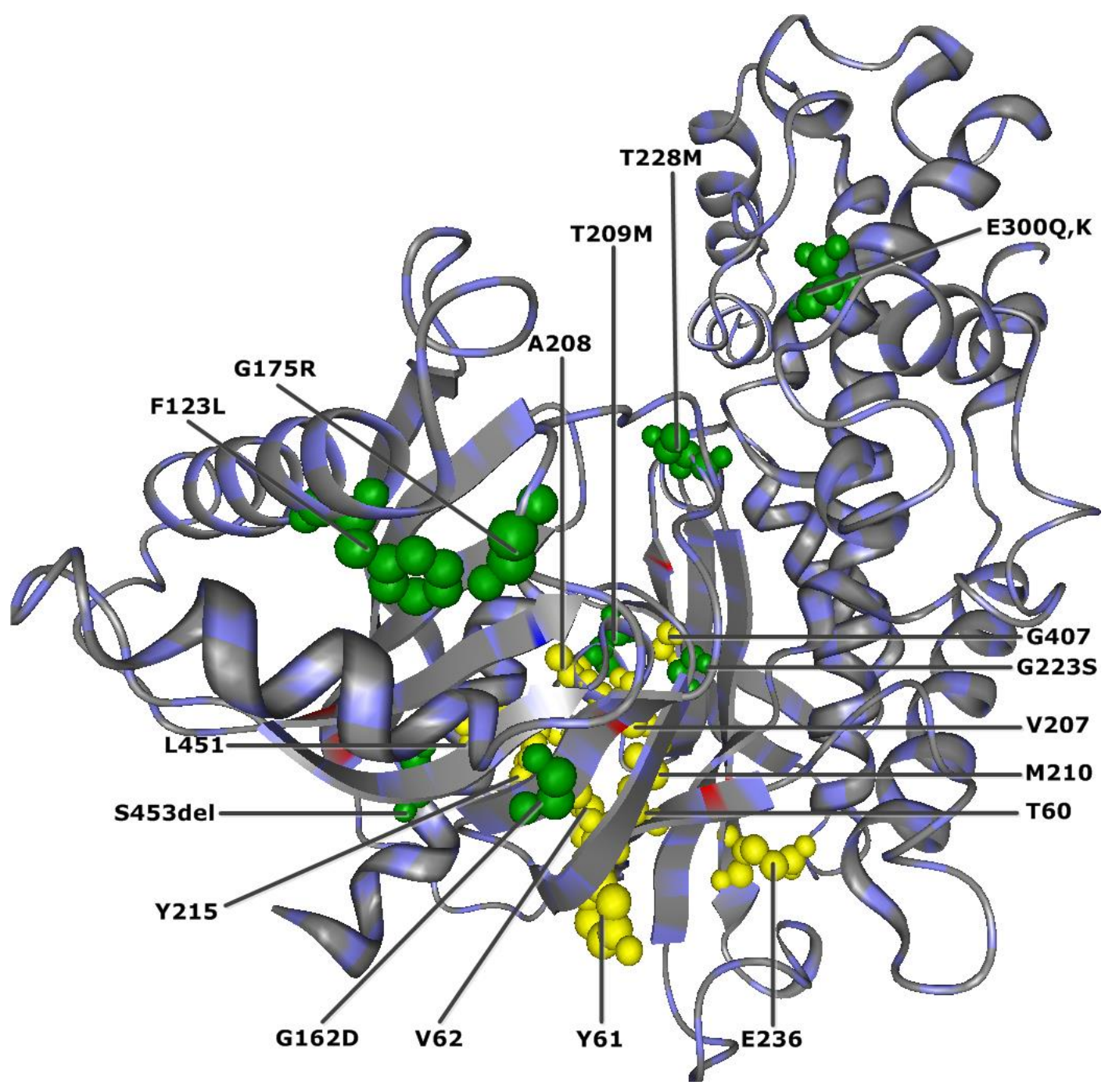

Figure 6.10: QCMF-significant positions that are either in contact with allosteric sites or related to nsSNPs in human GCK protein (PDB-Entry 1V4S). Yellow spheres correspond to structural localization of ten QCMF-significant residue sites which are in contact with allosteric sites where the V62, M210, Y215 are denoted as allosteric sites by themselves and they are also in contact with an other allosteric sites. Green spheres indicate eight QCMF-significant nsSNP positions in the GCK protein. Three of them (T209M, G223S and S453del) are further in contact with allosteric sites M210, I211, V452, V455 and A456. 
Moreover, when statistically evaluating both methods, we have observed that the new quantum information theory based method significantly outperforms the previous method. The new method reaches an improved performance in identifying essential sites from MSAs of both proteins with a significantly higher Matthews correlation coefficient (MCC) value of 0.215 whereas the previous CMF method reaches only a MCC value of 0.133. 


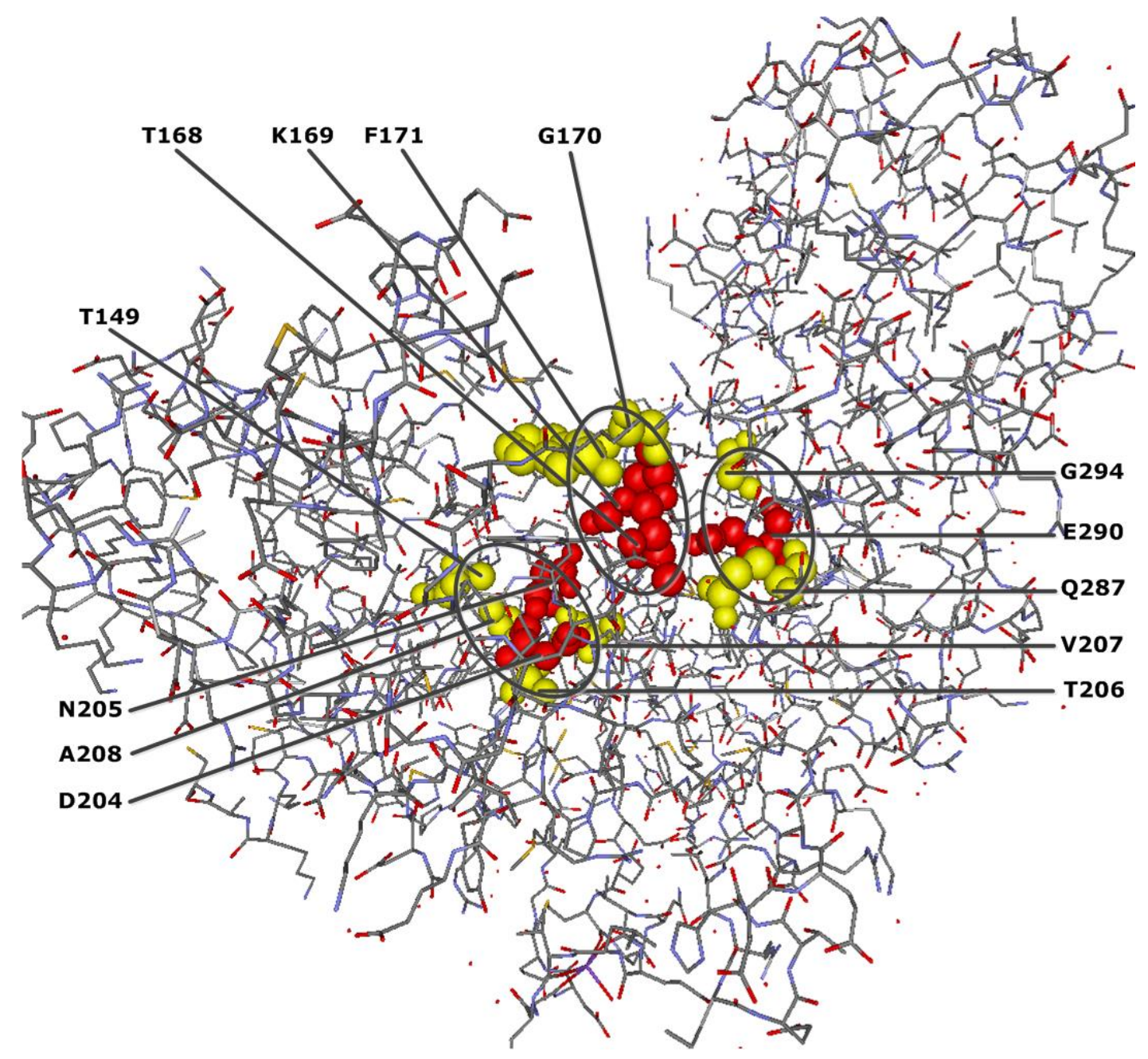

Figure 6.11: QCMF-significant residue positions are in contact with glucose binding site in human GCK protein (PDB-Entry 1V4S). (A) Red spheres show the structural positions of the glucose binding sites (active sites) and yellow spheres show the localization of QCMF-significant adjacent residue positions which are in contact with these active sites. The circles indicate clusters of glucose binding sites and their significant adjacent sites. 


\section{Discussion}

In this chapter, we will discuss the reason for setting 90-th percentile cut-off for the connectivity degree to evaluate results of our both classical and quantum information theory based methods. Afterwards, we discuss the results presented in the previous chapter in the context of related work.

\subsection{Connectivity Degree Cut-off}

The connectivity degree technique was introduced by Merkl and Zwick in [22] to characterize an individual residue site. In their study, they used the $\mathbb{U}$-metric, presented in the Section 4.1 and focused on only 75 columns pairs with the highest $\mathbb{U}(k, l)$-values as significant for each MSA. As a result of taking into account only certain number of significant column pair for each MSA, they set a fixed connectivity degree cut-off value of 3 for each MSA under investigation. It means if the connectivity degree of a residue site is equal or more than three, the residue is accepted as significant in [22].

However, after applying our MSA-specific significant model, only considering a fixed cutoff value for connectivity degree is too conservative and not suitable anymore. Hence, we have further developed the connectivity degree technique by utilizing a confidence interval method to identify a new cut-off value based on the Matthews correlation coefficient (MCC).

We have determined for the CMF and QCMF-significant residue sites going through all possible $n$-th percentiles for $n=80,81, \ldots, 99$, the MCC of a joint prediction for human EGFR and GCK protein. The maximal value of MCC is reached for CMF-significant sites if $n=90$ and likewise, the maximal value of MCC is reached if $n=88$ for QCMF-significant sites. Although we achieve the best MCC with $n=88$ for the QCMF-significant sites, we used in the result section 90\%-th percentile cut-off for the biological evaluation of these significant sites. In this way, we were able to compare both CMF and QCMF-significant residue sites fairly. In Table 7.1 and 7.2 we present the specificity, sensitivity and MCC values of all possible $n$-th percentiles for result of both methods. 


\begin{tabular}{|r|l|l|l|l|l|l|l|l|}
\hline Cut-off & Specificity & Sensitivity & MCC & Cut-off & Specificity & Sensitivity & MCC \\
\hline $99 \%$ & $99.53 \%$ & $3.16 \%$ & 0.086 & $89 \%$ & $86.38 \%$ & $23.11 \%$ & 0.112 \\
$98 \%$ & $97.65 \%$ & $5.10 \%$ & 0.065 & & $88 \%$ & $84.97 \%$ & $25.30 \%$ & 0.118 \\
$97 \%$ & $96.24 \%$ & $6.81 \%$ & 0.062 & & $87 \%$ & $82.15 \%$ & $26.52 \%$ & 0.096 \\
$96 \%$ & $94.83 \%$ & $9.00 \%$ & 0.068 & $86 \%$ & $79.34 \%$ & $27.73 \%$ & 0.077 \\
$95 \%$ & $93.42 \%$ & $10.70 \%$ & 0.067 & & $85 \%$ & $77.93 \%$ & $29.68 \%$ & 0.081 \\
$94 \%$ & $93.42 \%$ & $13.62 \%$ & 0.105 & $84 \%$ & $76.05 \%$ & $31.38 \%$ & 0.077 \\
$93 \%$ & $91.07 \%$ & $15.08 \%$ & 0.086 & $83 \%$ & $73.23 \%$ & $32.84 \%$ & 0.062 \\
$92 \%$ & $90.14 \%$ & $17.51 \%$ & 0.101 & & $82 \%$ & $70.89 \%$ & $34.06 \%$ & 0.050 \\
$91 \%$ & $88.73 \%$ & $19.22 \%$ & 0.101 & $81 \%$ & $69.01 \%$ & $35.76 \%$ & 0.047 \\
$\mathbf{9 0 \%}$ & $\mathbf{8 8 . 7 3 \%}$ & $\mathbf{2 2 . 1 4 \%}$ & $\mathbf{0 . 1 3 3}$ & $80 \%$ & $67.13 \%$ & $37.22 \%$ & 0.043 \\
\hline
\end{tabular}

Table 7.1: Statistical evaluation of CMF-significant residue sites for both human EGFR and GCK proteins. If connectivity degree cut-off is 90-percentile, our classical information theory based method reaches its maximal MCC-value.

\begin{tabular}{|r|l|l|l|l|r|l|l|l|}
\hline Cut-off & Specificity & Sensitivity & MCC & Cut-off & Specificity & Sensitivity & MCC \\
\hline $99 \%$ & $100 \%$ & $3.16 \%$ & 0.105 & $89 \%$ & $94.83 \%$ & $22.38 \%$ & 0.219 \\
$98 \%$ & $100 \%$ & $5.10 \%$ & 0.134 & & $\mathbf{8 8 \%}$ & $\mathbf{9 4 . 3 6 \%}$ & $\mathbf{2 4 . 3 3 \%}$ & $\mathbf{0 . 2 3 1}$ \\
$97 \%$ & $100 \%$ & $8.02 \%$ & 0.170 & $87 \%$ & $92.95 \%$ & $25.30 \%$ & 0.220 \\
$96 \%$ & $99.06 \%$ & $9.73 \%$ & 0.166 & & $86 \%$ & $91.54 \%$ & $26.52 \%$ & 0.212 \\
$95 \%$ & $98.12 \%$ & $11.43 \%$ & 0.165 & $85 \%$ & $90.14 \%$ & $27.49 \%$ & 0.203 \\
$94 \%$ & $97.65 \%$ & $13.86 \%$ & 0.182 & $84 \%$ & $89.67 \%$ & $28.22 \%$ & 0.204 \\
$93 \%$ & $97.18 \%$ & $15.81 \%$ & 0.194 & $83 \%$ & $89.20 \%$ & $29.68 \%$ & 0.212 \\
$92 \%$ & $96.24 \%$ & $17.51 \%$ & 0.195 & $82 \%$ & $88.73 \%$ & $31.87 \%$ & 0.226 \\
$91 \%$ & $96.24 \%$ & $19.22 \%$ & 0.211 & $81 \%$ & $87.32 \%$ & $32.84 \%$ & 0.218 \\
$90 \%$ & $95.30 \%$ & $21.163 \%$ & 0.215 & $80 \%$ & $86.38 \%$ & $34.54 \%$ & 0.222 \\
\hline
\end{tabular}

Table 7.2: Statistical evaluation of QCMF-significant residue sites for both human EGFR and GCK proteins. If connectivity degree cut-off is 88-percentile, our quantum information theory based method reaches its maximal MCC-value.

\subsection{CMF-significant residue sites}

Our results for human EGFR and GCK protein suggest that the large majority of CMFsignificant compensatory mutation sites are in agreement with previous experimental studies regarding the functions and stability of these proteins. 15 and $16 \mathrm{CMF}$-significant sites in human EGRF and GCK proteins, respectively, are verified as disease associated nsSNP positions (see Figures 6.3 and 6.5) where most amino acid substitutions in protein sequences 
damage structural stability of proteins [41,42,94]. Moreover, we have observed that in both proteins some of CMF-significant nsSNP positions are nearby allosteric sites, binding sites, or catalytic sites each of which are considered to be functionally important [95, 96]. Disease associated mutations at these nearby positions are likely to affect protein function [43, 97].

Despite the large number of CMF-significant sites demonstrated to be structurally or functionally important for both of the proteins, 9 and 15 significant sites in human EGFR and GCK proteins, respectively, are not included in the essential sites. However, we hypothesize that most of the novel significant sites may play a critical role in both proteins notwithstanding the absence of previous experimental data. Therefore, further progress from the molecular and structural biology end is required not only to assess the importance of these sites, but also for a future perspective on a deeper understanding of the protein structure.

Because we have also used the $\mathbb{U}$-metric, we compared our tool with H2r presented in [22] rather than with those methods developed in [23]. This way, we studied the impact of applying the Storey-Tibshirani procedure in combination with the effect of using the 90-th percentile cut-off for the connectivity degree. We have applied $\mathrm{H} 2 \mathrm{r}$ without adding pseudo counts to the human EGFR and GCK protein. For EGFR, the 14 sites T725, A755, N756, A767, Q791, V802, N816, V819, K846, V876, M881, K913, D916, and E931 are identified as significant. Out of these significant sites, ten of these residue sites T725, A755, N756, A767, Q791,K846, V876, M881, K913, and D916 are essential sites. On the other hand, for GCK, H2r identified the 15 residue positions L25, R36, R63, M107, C213, V226, G261, D262, G264, L266, D267, E268, T405, K414, and H416 as significant. Twelve of these sites, namely R36, R63, M107, C213, V226, G261,D262, G264, L266, D267, K414, and H416, are essential sites. However, when using the H2r Web service (http://wwwbioinf.uni-regensburg.de/) to analyze EGFR and GCK proteins, sensitivity is decreased, while precision is increased. By this service only eight sites for EGFR and nine sites for GCK were found to be significant. Moreover, only five and eight of them are verified as functionally or structurally important for EGFR and GCK proteins, respectively. This difference stems from the fact that the H2r Web service tightens the filtering of the columns. In addition to this, statistically evaluating H2r for EGFR and GCK proteins, we observed a sensitivity of $5.4 \%$, specificity of $96.7 \%$, and a MCC value of 0.047 .

The results of this comparison show that a vast majority of functionally or structurally important residue positions cannot be detected without using our novel MSA specific model and both metrics $\left(\mathbb{U}\right.$ and $\left.\mathbb{U}_{D(1)}\right)$ together.

\subsection{QCMF-significant residue sites}

The results of our new quantum information theory based method presented in the Section 6.3 show that the vast majority of QCMF-significant residue sites are closely related to functionality and structural stability of both human EGFR and GCK proteins. 10 signif- 
icant residue sites in EGFR and 19 significant sites in GCK are established as functionally important since they are directly located at or close to catalytic sites, allosteric sites and binding sites which are crucial for maintaining protein functions and for understanding the underlying molecular mechanism (see Figures 6.8, 6.9, 6.10, 6.11). Additionally, 2 significant sites in EGFR and 8 significant sites in GCK (three of them are also in contact with allosteric sites in GCK) are related to disease associated nsSNP regions of both proteins. As has been noted for the result of CMF-significant sites, most disease-causing mutations at these positions in corresponding sequences destroy structural features of proteins, thus affecting protein stability and often results in loss of protein function.

Although the importance of almost all QCMF-significant sites are verified through essential sites of both human proteins, there are still eight and two unconfirmed significant sites in EGFR and GCK proteins, respectively, which do not fall into essential sites. It is interesting to note that some of these unconfirmed sites are also referred as CMF-significant. We therefore believe that most of these unconfirmed sites identified by our present method may have an importance for the function and structural stability of both proteins notwithstanding the absence of previous experimental data. A further comparison reveals that the overlaps between the present QCMF-significant sites and previous CMF-significant sites are quite low, indicating that our both quantum and classical information theory based methods detect considerably different sets of residue sites as functionally and structurally important. The comparison result clearly shows that considering similar and dissimilar amino acid signals simultaneously, our present quantum information theory based method is more sensible to catalytic, allosteric and binding sites, while only focusing on dissimilar signals our previous classical information theory based method deals successfully with nsSNP positions in proteins. A final comparison between the methods is made based on their connectivity degree cut-off. As has been mentioned before, we initially set the connectivity degree cut-off value to the 90-th percentile at which the previous method reaches its maximal MCC value. However, going through all possible $n$-th percentiles for $n=80,81, \ldots, 99$, our present method reaches its maximal MCC value of 0.231 if $n=88$ (see Table 7.2).

Although the QCMF shows a better performance than CMF, the comparison study indicates that the result of the QCMF complements the result of CMF. The simultaneous usage of both methods can significantly improve the identification of important sites of proteins. 


\section{Conclusion}

In the final chapter of this thesis, we summarize our findings and contributions. Furthermore, we give an outlook on future work in this research direction.

\subsection{Summary}

In this thesis, we reported two new methods based on classical information theory and quantum information theory for the identification of functionally or structurally important non-conserved residue sites by analyzing compensatory mutations in MSAs. In addition, we developed an MSA-specific statistical model by slightly generalizing the Storey-Tibshirani procedure devised for multiple testing problems. This model separates method-based significant compensatory mutation signals from background noise and quantifies further the error made in terms of the false discovery rate.

Our first method (see Chapter 4) is based on classical information theory and includes two metrics. While the first metric (developed by Merkl and Zwick in [22]) does not consider the biochemical constraints of amino acids, the second metric focuses on significant and BLOSUM62-dissimilar amino acid signals as a model of compensatory mutations. In addition, these signals are integrated in calculation of its metric using a doubly stochastic matrix to transform the empirical pair distribution of the column pair. Using this approach, we can show how dissimilar compensatory mutations have affected genomic sequences in the course of evolution.

In contrast to the first method, applying principles of quantum information theory, our second method (see Chapter 5) simultaneously models similar and dissimilar amino acid pair signals in the detection of functionally or structurally important sites. This method also includes two metrics: the first metric measures compensatory mutations between pairs of columns; the second metric considers the sequence conservation of columns.

Applying the MSA-specific statistical model for our classical and quantum information theory based methods, we determined for each method as well as each metric the amount of significant compensatory mutation signals in MSAs of two human proteins. To demonstrate the performance and functionality of our methods, we analyzed the structurally or functionally important positions of these proteins. The results show that overlaps between two information theory based methods are quite low, indicating that both methods detect considerably different sets of residue sites as functionally and structurally important. Only 
focusing on dissimilar signals, the first method successfully deals with nsSNP positions. In contrast, simultaneously considering similar and dissimilar amino acid signals, our second method is more sensible to catalytic, allosteric and binding sites than those found by the first method.

\subsection{Outlook}

Although we developed two information theory based methods and one statistical model for the identification of functionally and structurally important sites of proteins, there is still need for a method to replace the 90-th percentile cut-off by an MSA-dependent threshold for the connectivity degree. The extension of the connectivity degree technique would provide opportunities for future studies, which could also be applied for several biological network analysis.

Another interesting extension of this thesis would be the construction of the matrices, introduced in Section 4.3, taking into account only either functionally or structurally important sites in MSAs. In this thesis, we constructed these matrices simultaneously using functionally and structurally important sites. However, considering them individually for the matrix construction could provide us to measure the effect of biochemical constraints of amino acids in the identification of important residue sites.

Furthermore, in order to get an improved performance in identifying important sites in proteins, our both classical information theory and quantum information theory based methods could be combined using a machine learning model, such as random forests, support vector machine, or other comparable models. The combination of both methods with a suitable machine learning model would result in a significantly better performance for the identification of important sites. 



\section{Bibliography}

[1] Nelson DL, MCox M: Lehninger Prenciples of Biochemistry. New York, NY 10010: W.H Freeman and Company, 41 Madison Avenue, fifth edition edition 2008.

[2] Berman HM, Westbrook J, Feng Z, Gilliland G, Bhat TN, Weissig H, Shindyalov IN, Bourne PE: The Protein Data Bank. Nucleic Acids Research 2000, 28:235-242, [http://nar.oxfordjournals.org/content/28/1/235.abstract]

[3] Gloor GB, Martin LC, Wahl LM, Dunn SD: Mutual Information in Protein Multiple Sequence Alignments Reveals Two Classes of Coevolving Positions. Biochemistry 2005, 44(19):7156-7165, [http://pubs.acs.org/doi/abs/10.1021/ bi050293e]. [PMID: 15882054].

[4] Gültas M, Haubrock M, Tüysüz N, Waack S: Coupled Mutation Finder: A new entropy-based method quantifying phylogenetic noise for the detection of compensatory mutations. BMC Bioinformatics 2012, 13:225, [http://www . biomedcentral.com/1471-2105/13/225]

[5] Gültas M, Düzgün G, Herzog S, Jäger S, Meckbach C, Wingender E, Waack S: Quantum coupled mutation finder: predicting functionally or structurally important sites in proteins using quantum Jensen-Shannon divergence and CUDA programming. BMC Bioinformatics 2014, 15:96, [http://www . biomedcentral . com/1471-2105/15/96]

[6] Wilson K, Walker J: Principles and Techniques of Biochemistry and Molecular Biology. Cambridge University Press, 7th edition 2010.

[7] Altschuh D, Lesk AM, Bloomer AC, Klug A: Correlation of co-ordinated amino acid substitutions with function in viruses related to tobacco mosaic virus. Journal of Molecular Biology 1987, 193(4):693-707.

[8] Martin LC, Gloor GB, Dunn SD, Wahl LM: Using information theory to search for co-evolving residues in proteins. Bioinformatics 2005, 21(22):4116-4124, [http: //bioinformatics.oxfordjournals.org/content/21/22/4116.abstract]

[9] Dunn SD, Wahl LM, Gloor GB: Mutual information without the influence of phylogeny or entropy dramatically improves residue contact prediction. Bioinformatics 2008, 24(3):333-340. 
[10] Chakrabarti S, Panchenko AR: Structural and Functional Roles of Coevolved Sites in Proteins. PLoS ONE 2010, 5:e8591, [http://dx.doi.org/10.1371\% 2Fjournal.pone.0008591].

[11] Sandler I, Abu-Qarn M, Aharoni A: Protein co-evolution: how do we combine bioinformatics and experimental approaches? Mol. BioSyst. 2013, 9:175-181, [http://dx.doi.org/10.1039/C2MB25317H].

[12] DePristo MA, Weinreich DM, Hartl DL: Missense meanderings in sequence space: a biophysical view of protein evolution. Nat Rev Genet Nature Publishing Group 2005, 6(9):678-687, [http://dx.doi.org/10.1038/nrg1672].

[13] Yeang $\mathrm{CH}$, Haussler D: Detecting Coevolution in and among Protein Domains. PLoS Comput Biol 2007, 3(11):e211, [http://dx.plos.org/10.1371\% 2Fjournal.pcbi.0030211]

[14] Goh CS, Bogan AA, Joachimiak M, Walther D, Cohen FE: Co-evolution of proteins with their interaction partners. Journal of Molecular Biology 2000, 299(2):283 - 293, [http://www.sciencedirect.com/science/article/pii/ S002228360093732X].

[15] Lockless SW, Ranganathan R: Evolutionarily Conserved Pathways of Energetic Connectivity in Protein Families. Science 1999, 286(5438):295-299, [http:// www. sciencemag.org/content/286/5438/295.abstract].

[16] Göbel U, Sander C, Schneider R, Valencia A: Correlated mutations and residue contacts in proteins. PROTEINS-STRUCTURE FUNCTION AND GENETICS 1994, 18(4):309-317.

[17] Neher E: How frequent are correlated changes in families of protein sequences? Proceedings of the National Academy of Sciences 1994, 91:98-102, [http://www. pnas.org/content/91/1/98.abstract].

[18] Pollock DD, Taylor WR: Effectiveness of correlation analysis in identifying protein residues undergoing correlated evolution. Protein Engineering 1997, 10(6):647657,[http://peds.oxfordjournals.org/content/10/6/647.abstract].

[19] Dekker JP, Fodor A, Aldrich RW, Yellen G: A perturbation-based method for calculating explicit likelihood of evolutionary co-variance in multiple sequence alignments. Bioinformatics 2004, 20(10):1565-1572.

[20] Atchley, W R and Wollenberg, K R and Fitch, W M and Terhalle, W and Dress, A W: Correlations among amino acid sites in bHLH protein domains: an information theoretic analysis. Mol. Biol. Evol. 2000, 17:164. 
[21] Tillier ER, Lui TW: Using multiple interdependency to separate functional from phylogenetic correlations in protein alignments. Bioinformatics 2003, 19(6):750-755, [http://bioinformatics.oxfordjournals.org/content/19/ 6/750.abstract].

[22] Merkl R, Zwick M: H2r: Identification of evolutionary important residues by means of an entropy based analysis of multiple sequence alignments. BMC Bioinformatics 2008, 9:151, [http://www. biomedcentral.com/1471-2105/9/151]

[23] Gao H, Dou Y, Yang J, Wang J: New methods to measure residues coevolution in proteins. BMC Bioinformatics 2011, 12:206, [http://www.biomedcentral.com/ 1471-2105/12/206].

[24] Codoner FM, Fares M: Why Should We Care About Molecular Coevolution? Evolutionary Bioinformatics 2008, 4:29-38.

[25] Noivirt O, Eisenstein M, Horovitz A: Detection and reduction of evolutionary noise in correlated mutation analysis. Protein Engineering Design and Selection May 2005, 18(5):247-253, [http://peds.oxfordjournals.org/content/18/ 5/247.abstract].

[26] Wollenberg KR, Atchley WR: Separation of phylogenetic and functional associations in biological sequences by using the parametric bootstrap. Proceedings of the National Academy of Sciences 2000, 97(7):3288-3291, [http://www.pnas.org/ content $/ 97 / 7 / 3288$.abstract].

[27] Storey JD, Tibshirani R: Statistical significance for genomewide studies. Proc. Acad. Sci. 2003, 100:9440-9445.

[28] Walsh B: Multiple comparisons: Bonferroni Corrections and False Discovery Rates. Lecture Notes EEB 581, Department of Ecology and Evolutionary Biology, University of Arizona 2004.

[29] Dixit A, Yi L, Gowthaman R, Torkamani A, Schork NJ, Verkhivker GM: Sequence and Structure Signatures of Cancer Mutation Hotspots in Protein Kinases. PLoS ONE 2009, 4(10):e7485, [http://dx.doi.org/10.1371\% 2Fjournal.pone.0007485].

[30] Yun CH, Boggon TJ, Li Y, Woo MS, Greulich H, Meyerson M, Eck MJ: Structures of Lung Cancer-Derived EGFR Mutants and Inhibitor Complexes: Mechanism of Activation and Insights into Differential Inhibitor Sensitivity. Cancer Cell 2007, 11(3):217 - 227, [http://www.sciencedirect.com/science/article/ pii/S1535610807000281]. 
[31] Zhang H, Berezov A, Wang Q, Zhang G, Drebin J, Murali R, Greene MI: ErbB receptors: from oncogenes to targeted cancer therapies. The Journal of Clinical Investigation 2007, 117(8):2051-2058, [http://www.jci.org/articles/view/ 32278].

[32] Lynch TJ, Bell DW, Sordella R, Gurubhagavatula S, Okimoto RA, Brannigan BW, Harris PL, Haserlat SM, Supko JG, Haluska FG, Louis DN, Christiani DC, Settleman J, Haber DA: Activating Mutations in the Epidermal Growth Factor Receptor Underlying Responsiveness of Non-Small-Cell Lung Cancer to Gefitinib. New England Journal of Medicine 2004, 350(21):2129-2139, [http://www.nejm.org/doi/ full/10.1056/NEJMoa040938].

[33] Balius TE, Rizzo RC: Quantitative Prediction of Fold Resistance for Inhibitors of EGFR. Biochemistry 2009, 48(35):8435-8448, [http://pubs.acs.org/doi/ abs/10.1021/bi900729a], [PMID: 19627157].

[34] Tinto N, Zagari A, Capuano M, De Simone A, Capobianco V, Daniele G, Giugliano M, Spadaro R, Franzese A, Sacchetti L: Glucokinase Gene Mutations: Structural and Genotype-Phenotype Analyses in MODY Children from South Italy. PLoS ONE 2008, 3(4):e1870, [http://dx.plos.org/10.1371\% 2Fjournal.pone.0001870].

[35] Capuano M, Garcia-Herrero CM, Tinto N, Carluccio C, Capobianco V, Coto I, Cola A, Iafusco D, Franzese A, Zagari A, Navas MA, Sacchetti L: Glucokinase (GCK) Mutations and Their Characterization in MODY2 Children of Southern Italy. PLoS ONE 2012, 7(6):e38906, [http://dx.doi .org/10.1371\%2F journal. pone.0038906].

[36] Garcia-Herrero CM, Rubio-Cabezas O, Azriel S, Gutierrez-Nogues A, Aragones A, Vincent O, Campos-Barros A, Argente J, Navas MA: Functional Characterization of MODY2 Mutations Highlights the Importance of the Fine-Tuning of Glucokinase and Its Role in Glucose Sensing. PLoS ONE 2012, 7:e30518, [http://dx.doi. org $/ 10.1371 \% 2$ Fjournal.pone.0030518].

[37] Kamata K, Mitsuya M, Nishimura T, ichi Eiki J, Nagata Y: Structural Basis for Allosteric Regulation of the Monomeric Allosteric Enzyme Human Glucokinase. Structure 2004, 12(3):429 - 438, [http://www.sciencedirect.com/science/ article/pii/S0969212604000474]

[38] Hamosh A, Scott AF, Amberger JS, Bocchini CA, McKusick VA: Online Mendelian Inheritance in Man (OMIM), a knowledgebase of human genes and genetic disorders. Nucleic Acids Research 2005, 33(suppl 1):D514-D517, [http://nar. oxfordjournals.org/content/33/suppl_1/D514.abstract] 
[39] Reichert J, Sühnel J: The IMB Jena Image Library of Biological Macromolecules: 2002 update. Nucleic Acids Research 2002, 30:253-254, [http://nar. oxfordjournals.org/content/30/1/253.abstract]

[40] Flicek P, Amode MR, Barrell D, Beal K, Brent S, Chen Y, Clapham P, Coates G, Fairley S, Fitzgerald S, Gordon L, Hendrix: Ensembl 2011. Nucleic Acids Research 2011, 39(suppl 1):D800-D806, [http://nar.oxfordjournals.org/content/ 39/suppl_1/D800.abstract]

[41] Sunyaev S, Ramensky V, Bork P: Towards a structural basis of human non-synonymous single nucleotide polymorphisms. Trends in Genetics 2000, 16(5):198 - 200, [http://www.sciencedirect.com/science/article/pii/ S0168952500019880].

[42] Wang Z, Moult J: SNPs, protein structure, and disease. Human Mutation 2001, 17(4):263-270, [http://dx.doi.org/10.1002/humu.22].

[43] Burke D, Worth C, Priego EM, Cheng T, Smink L, Todd J, Blundell T: Genome bioinformatic analysis of nonsynonymous SNPs . BMC Bioinformatics 2007, 8:301, [http://www.biomedcentral.com/1471-2105/8/301].

[44] Xiong J: Essential Bioinformatics. New York: Cambridge Universtiy Press 2006.

[45] Lesk AM: Introduction To Bioinformatics. Oxford University Press, second edition edition 2005.

[46] Lodish H: Molecular Cell Biology. W. H. Freeman, W.H. Freeman 2012, [http: //books.google.de/books?id=hdn7ngEACAAJ].

[47] Mount DW: Bioinformatics: Sequence and Genome Analysis. Cold Spring Harbor Laboratory Press, New York, second edition edition 2001.

[48] Hansen B, Jorde LB: USMLE Step 1 Lecture Notes Biochemistry. KAPLAN medical 2004.

[49] Barnes MR, Gray IC: Bioinformatics for Geneticists. West Sussex PO19 8SQ, England: John Wiley and Sons Ltd, 2003.

[50] Wang S: On Multiple Sequence Alignment. Dissertation, The University of Texas at Austin 2007.

[51] D H: Bioinformatics 1, Multiple Sequence Alignment. Tech. rep., Algorithms in Bioinformatics, University of Tuebingen 2009. 
[52] Yun CH, Mengwasser KE, Toms AV, Woo MS, Greulich H, Wong KK, Meyerson M, Eck MJ: The T790M mutation in EGFR kinase causes drug resistance by increasing the affinity for ATP. Proceedings of the National Academy of Sciences 2008, 105(6):2070-2075, [http://www.pnas.org/content/105/6/2070.abstract].

[53] Protein Data Bank Contents Guide: Atomic Coordinate Entry Format Description. $w w P D B$ 2012, Version 3.30.

[54] Schneider R, Sander C: The HSSP Database of Protein StructureSequence Alignments. Nucleic Acids Research 1996, 24:201-205, [http: //nar.oxfordjournals.org/content/24/1/201.abstract].

[55] Bairoch A, Boeckmann B: The SWISS-PROT protein sequence data bank. Nucleic Acids Research 1992, 20(suppl):2019-2022, [http://nar.oxfordjournals.org/ content/20/supplement/2019.short].

[56] Kabsch W, Sander C: Dictionary of protein secondary structure: Pattern recognition of hydrogen-bonded and geometrical features. Biopolymers 1983, 22(12):2577-2637, [http://dx.doi.org/10.1002/bip.360221211]

[57] Brown NP, Leroy C, Sander C: MView: a web-compatible database search or multiple alignment viewer. Bioinformatics 1998, 14(4):380-381, [http:// bioinformatics.oxfordjournals.org/content/14/4/380.abstract]

[58] Porter CT, Bartlett GJ, Thornton JM: The Catalytic Site Atlas: a resource of catalytic sites and residues identified in enzymes using structural data. Nucleic Acids Research 2004, 32(suppl 1):D129-D133, [http://nar.oxfordjournals. org/content/32/suppl_1/D129.abstract].

[59] Laskowski RA: PDBsum: summaries and analyses of PDB structures. Nucleic Acids Research 2001, 29:221-222, [http://nar.oxfordjournals.org/ content/29/1/221. abstract].

[60] Laskowski RA, Chistyakov VV, Thornton JM: PDBsum more: new summaries and analyses of the known 3D structures of proteins and nucleic acids. Nucleic Acids Research 2005, 33(suppl 1):D266-D268, [http://nar.oxfordjournals. org/content/33/suppl_1/D266.abstract].

[61] Holland RCG, Down TA, Pocock M, Prlic A, Huen D, James K, Foisy S, Dreager A, Yates A, Heuer M, Schreiber MJ: BioJava: an open-source framework for bioinformatics. Bioinformatics 2008, 24(18):2096-2097, [http://bioinformatics . oxfordjournals.org/content/24/18/2096.abstract] 
[62] Keskin O, Tsai CJ, Wolfson H, Nussinov R: A new, structurally nonredundant, diverse data set of protein-protein interfaces and its implications. Protein Science 2004, 13(4):1043-1055, [http://dx.doi.org/10.1110/ps.03484604]

[63] Deza MM, Deza E: Encyclopedia of Distances. Springer Dordrecht Heidelberg London New York 2009.

[64] Henikoff S, Henikoff JG: Amino acid substitution matrices from protein blocks. Proceedings of the National Academy of Sciences 1992, 89(22):10915-10919, [http: //www.pnas.org/content/89/22/10915.abstract]

[65] Pietrokovski S, Henikoff JG, Henikoff S: The Blocks Database A System for Protein Classification. Nucleic Acids Research 1996, 24:197-200, [http://nar. oxfordjournals.org/content/24/1/197.abstract]

[66] Cover TM, Thomas JA: Elements of Information Theory. John Wiley \& Sons, Inc, second edition edition 2006.

[67] Desurvire E: Classical and Quantum Information Theory An Indroduction for the Telecom Scientist. Cambridge University Press 2009.

[68] Vedral V: Introduction to Quantum Information Science. Oxford University Press 2006.

[69] Nielsen MA, Chuang IL: Quantum Computation and Quantum Information. Cambridge University Press 2000.

[70] Wilde MM: Quantum Information Theory. Cambridge University Press 2013.

[71] McMahon D: Quantum Computing Explained . John Wiley and Sons Ltd, 2007.

[72] Lin J: Divergence measures based on the Shannon entropy. Information Theory, IEEE Transactions on 1991, 37:145-151.

[73] Fuglede B, Topsoe F: Jensen-Shannon divergence and Hilbert space embedding. In Information Theory, 2004. ISIT 2004. Proceedings. International Symposium on 2004:31.

[74] Ewens WJ, Grant GR: Statistical Methods in Bioinformatics: An Introduction. Springer-Verlag New York 2001.

[75] Benjamini Y, Hochberg Y: Controlling the False Discovery Rate: A Practical and Powerful Approach to Multiple Testing. Journal of the Royal Statistical Society. Series B (Methodological) 1995, 57:pp. 289-300, [http://www . jstor.org/stable/ 2346101]. 
[76] Ferreira JA, Zwinderman AH: On the Benjamini-Hochberg Method. The Annals of Statistics 2006, 34(4):pp. 1827-1849, [http://www.jstor.org/stable/ 25463486]

[77] Ferreira JA, Zwinderman AH: Approximate Power and Sample Size Calculations with the Benjamini-Hochberg Method. The International Journal of Biostatistics 2006, 2.

[78] Bremm S, Schreck T, Boba P, Held S, Hamacher K: Computing and visually analyzing mutual information in molecular co-evolution. BMC Bioinformatics 2010, 11:330, [http://www.biomedcentral.com/1471-2105/11/330]

[79] Dieter U, Ahrens JH: Acceptance-Rejection Techniques For Sampling From The Gamma And Beta Distribution. Tech. rep., Stanford University 1974.

[80] Wang G, Jr RLD: PISCES: recent improvements to a PDB sequence culling server. Nucleic Acids Research 2005, 33(Web-Server-Issue):94-98.

[81] Eyal E, Frenkel-Morgenstern M, Sobolev V, Pietrokovski S: A pair-to-pair amino acids substitution matrix and its applications for protein structure prediction. Proteins: Structure, Function, and Bioinformatics 2007, 67:142-153, [http://dx. doi.org/10.1002/prot.21223].

[82] Asper RY: Classifiers for Discrimination of Significant Protein Residues and Protein-Protein Interaction Using Concepts of Information Theory and Machine Learning. Dissertation, Georg-August-University of Goettingen 2011.

[83] Cappellini V, Sommer HJ, Bruzda W, Zyczkowski K: Random bistochastic matrices. J.Phys. A: Math. Theor. 2009, 42:23.

[84] Birkhoff G: Tres observationes sobre et algebra lineal. Univ. Nac. Tucaman Rev. 1946, A(5).

[85] Hardy G, Littlewood J, Pólya G: Inequalities. Oxford: Oxford University Press, 2nd edition 1952.

[86] Johansson F, Toh H: Relative von Neumann entropy for evaluating amino acid conservation. Journal of Bioinformatics and Computational Biology 2010, 08(05):809-823, [http://www.worldscientific.com/doi/abs/10.1142/ S021972001000494X].

[87] Grosse I, Bernaola-Galván P, Carpena P, Román-Roldán R, Oliver J, Stanley HE: Analysis of symbolic sequences using the Jensen-Shannon divergence. Phys. Rev. E 2002, 65:041905, [http://link.aps.org/doi/10.1103/PhysRevE.65. 041905]. 
[88] Capra JA, Singh M: Predicting functionally important residues from sequence conservation. Bioinformatics 2007, 23(15):1875-1882.

[89] Caffrey DR, Somaroo S, Hughes JD, Mintseris J, Huang ES: Are protein-protein interfaces more conserved in sequence than the rest of the protein surface? Protein Science 2004, 13:190-2002.

[90] Herbst RS: Review of epidermal growth factor receptor biology. International Journal of Radiation Oncology*Biology*Physics 2004, 59(2, Supplement):S21 - S26, [http://www.sciencedirect.com/science/article/pii/ S0360301604003311].

[91] Thornton PS, Satin-Smith MS, Herold K, Glaser B, Chiu KC, Nestorowicz A, Permutt M, Baker L, Stanley CA: Familial hyperinsulinism with apparent autosomal dominant inheritance: Clinical and genetic differences from the autosomal recessive variant. The Journal of Pediatrics 1998, 132:9 - 14, [http://www . sciencedirect.com/science/article/pii/S0022347698704779].

[92] Sherry ST, Ward MH, Kholodov M, Baker J, Phan L, Smigielski EM, Sirotkin K: dbSNP: the NCBI database of genetic variation. Nucleic Acids Research 2001, 29:308-311, [http://nar.oxfordjournals.org/content/29/1/ 308.abstract]

[93] Valentinova L, Beer NL, Stanik J, Tribble ND, van de Bunt M, Huckova M, Barrett A, Klimes I, Gasperikova D, Gloyn AL: Identification and Functional Characterisation of Novel Glucokinase Mutations Causing Maturity-Onset Diabetes of the Young in Slovakia. PLoS ONE 2012, 7(4):e34541, [http://dx.doi.org/10. 1371\%2F journal.pone.0034541].

[94] Cheng TMK, Lu YE, Vendruscolo M, Lio' P, Blundell TL: Prediction by Graph Theoretic Measures of Structural Effects in Proteins Arising from Non-Synonymous Single Nucleotide Polymorphisms. PLoS Comput Biol 2008, 4(7):e1000135, [http: //dx.doi.org/10.1371\%2Fjournal.pcbi.1000135]

[95] Bao L, Cui Y: Functional impacts of non-synonymous single nucleotide polymorphisms: Selective constraint and structural environments. FEBS Letters 2006, 580(5):1231 - 1234, [http://www.sciencedirect.com/science/ article/pii/S0014579306000755].

[96] $\mathrm{Ng}$ PC, Henikoff S: SIFT: predicting amino acid changes that affect protein function. Nucleic Acids Research 2003, 31(13):3812-3814, [http://nar. oxfordjournals.org/content/31/13/3812. abstract]. 
[97] Ramensky V, Bork P, Sunyaev S: Human non-synonymous SNPs: server and survey. Nucleic Acids Research 2002, 30(17):3894-3900, [http://nar. oxfordjournals.org/content/30/17/3894.abstract] 



\section{Appendix}

9.1 Appendix A: Coupled Mutation Finder 


\title{
Coupled mutation finder: A new entropy- based method quantifying phylogenetic noise for the detection of compensatory mutations
}

\author{
Mehmet Gültas ${ }^{1 *}$, Martin Haubrock ${ }^{2}$, Nesrin Tüysüz ${ }^{3}$ and Stephan Waack ${ }^{1 *}$
}

\begin{abstract}
Background: The detection of significant compensatory mutation signals in multiple sequence alignments (MSAs) is often complicated by noise. A challenging problem in bioinformatics is remains the separation of significant signals between two or more non-conserved residue sites from the phylogenetic noise and unrelated pair signals. Determination of these non-conserved residue sites is as important as the recognition of strictly conserved positions for understanding of the structural basis of protein functions and identification of functionally important residue regions. In this study, we developed a new method, the Coupled Mutation Finder (CMF) quantifying the phylogenetic noise for the detection of compensatory mutations.

Results: To demonstrate the effectiveness of this method, we analyzed essential sites of two human proteins: epidermal growth factor receptor (EGFR) and glucokinase (GCK). Our results suggest that the CMF is able to separate significant compensatory mutation signals from the phylogenetic noise and unrelated pair signals. The vast majority of compensatory mutation sites found by the CMF are related to essential sites of both proteins and they are likely to affect protein stability or functionality.

Conclusions: The CMF is a new method, which includes an MSA-specific statistical model based on multiple testing procedures that quantify the error made in terms of the false discovery rate and a novel entropy-based metric to upscale BLOSUM62 dissimilar compensatory mutations. Therefore, it is a helpful tool to predict and investigate compensatory mutation sites of structural or functional importance in proteins. We suggest that the CMF could be used as a novel automated function prediction tool that is required for a better understanding of the structural basis of proteins. The CMF server is freely accessible at http://cmf.bioinf.med.uni-goettingen.de.
\end{abstract}

\section{Background}

A multiple sequence alignment (MSA) of proteins contains a set of aligned amino acid sequences in which homologous residues of different sequences are placed in same columns. Therefore, functionally or structurally important amino acids and their positions both of which are often strictly conserved are easily detectable with MSAs [1-3]. On the other hand, detection of important non-conserved residue positions related to several

\footnotetext{
*Correspondence: gueltas@cs.uni-goettingen.de;

waack@cs.uni-goettingen.de

${ }^{1}$ Institute of Computer Science, University of Göttingen, Goldschmidtstr. 7, 37077, Göttingen, Germany
}

Full list of author information is available at the end of the article essential conserved residues requires a more sophisticated approach. The usage of methods such as correlation analysis allow the identification of important nonconserved residue positions based on their correlated mutation manners $[4,5]$ due to functional coupling of mutation positions. This coupling might stem from one mutation in a certain site affecting a compensating mutation at another site, even if both related residue sites are distantly positioned in the protein structure. Moreover, these coupled mutations can result from spatial, physical, or chemical restrictions or signaling of allostery $[4,5]$. Thus, determination of these positions is as crucial as the recognition of strictly conserved positions for the understanding of the structural basis of protein functions, and for the identification of functionally important residue regions which might be disease associated, responsible for 
the maintenance of internal protein volume, or possibly form key sites for interactions within or between proteins [6-9].

Until now, a variety of studies have employed Pearson's correlation coefficient methods [10-12], perturbation based methods $[9,13]$ and mutual information (MI) based methods [6,14-17] because of their simplicity and efficiency for the detection of coupled mutations in MSAs. However, due to background noise, all of these methods interfere with the identification of compensatory mutation signals $[14,18,19]$. Hence, the significant compensatory mutation signals must be separated from the background noise that might occur as a result of: i) false signals arising from insufficient data; ii) sites with low or high conservation biasing the signal; iii) phylogenetic noise. While the first two types of noise can be easily overcome by appropriately filtering the data [16], phylogenetic noise can only be eliminated to some extent by excluding highly similar sequences from the MSA [19].

Recently, several methods such as bootstrapping, simulation or randomization methods have been utilized in order to minimize the influence of phylogenetic linkage and stochastic noise [15,20,21]. Dunn et al. [19] have introduced the average product correction (APC), to adjust MI for background effects. Merkl and Zwick, in their study, [16] have used a normalized MI (see Equation 1) and focused on only 75 residue pairs with the highest normalized MI values as significant for each MSA. Gao et al. [17] have pursued a similar approach, where they have replaced the metric used in [16] with the amino acid background distribution (MIB). While the reduction of background noise in the model of Dunn et al. is not quantified, the approaches of Gao et al. and Merkl and Zwick appear to be over-conservative, yet specific.

Despite the presence of a variety of different methods as mentioned above, to date there is still need for a method to deal with the noise as well as for powerful metrics to improve the sensitivity. In this study, we have developed such a method called Coupled Mutation Finder (CMF). The CMF includes an MSA-specific statistical model based on multiple testing procedures described in $[22,23]$ and a novel entropy-based metric to upscale dissimilar compensatory mutations and also to complement the normalized MI metric used in [16]. Unlike previous normalized MI based studies [16,17], we have separated metric-based significant compensatory mutation signals from background noise with respect to our MSA specific statistical model that quantifies the error made in terms of the false discovery rate.

To demonstrate the performance and functionality of the CMF, we analyzed the structurally or functionally important positions of two human proteins, namely epidermal growth factor receptor (EGFR) and glucokinase (GCK). The proteins have been chosen because their functionally and structurally important positions have been experimentally well studied previously [24-35]. As a result, the $C M F$ detects in these two proteins disease associated amino acid mutations (non-synonymous single nucleotide polymorphisms (nsSNPs)), not strictly conserved catalytic or binding sites, and residues that are nearby one of these sites or in the close neighborhood of a strictly conserved positions, which are likely to affect protein stability or functionality [36-38].

\section{Results}

Our method to predict functionally or structurally important residue positions is composed of two steps. First, we have devised a new MSA-specific statistical method for the identification of significant MSA column pairs with respect to either of the two metrics $\mathbb{U}$ and $\mathbb{U}_{D(\alpha)}$. Assume that $M$ is the MSA under study, these pairs are annotated as $(\mathbb{U}, M)$-significant and $\left(\mathbb{U}_{D(\alpha)}, M\right)$-significant, respectively. Second, we utilized the connectivity degree of a residue site with respect to a metric introduced in [16]. The connectivity degree of a residue site indicates the number of its significant coupled mutation pairs. In this case, a site is called (U,M)-significant when the frequency of occurrence of this site in the set of $(\mathbb{U}, M)$-significant pairs exceeds the 90-th percentile. Having defined the concept of a $\left(\mathbb{U}_{D(\alpha)}, M\right)$-significant site analogously, a site is defined as $C M F$-significant with respect to $M$, when it is either $(\mathbb{U}, M)$-significant or $\left(\mathbb{U}_{D(\alpha)}, M\right)$-significant.

In this study, we analyzed human EGFR (pdb entry 2J6M) and GCK (pdb entry 1V4S) proteins with a false discovery rate $(F D R)$ of $1 \%$. For the preceding one, we defined a total of 14339 out of 26079 non-conserved column pairs as significant. 11365 of these significant pairs are detected as $(\mathbb{U}, M)$-significant and 3798 pairs are observed as $\left(\mathbb{U}_{D(\alpha)}, M\right)$-significant. Only 824 EGFR pairs are significant with respect to both metrics. On the other hand, for GCK, we identified a total of 32654 out of 69645 non-conserved column pairs as significant where 18106 of them are $\mathbb{U}$-significant and 16241 are $\mathbb{U}_{D(1)}$-significant. Moreover, 1693 pairs are defined as significant for both $\mathbb{U}$ and $\mathbb{U}_{D(1)}$-significant.

Applying the connectivity degree technique, we identified 22 and 36 residue positions as U-significant for human EGFR and GCK proteins, respectively. Additionally, 21 positions of EGFR and 36 positions of GCK were detected as $\mathbb{U}_{D(1)}$-significant. Finally, a total of 43 sites of EGFR and 72 of GCK were found as $C M F$-significant, and predicted as of structural or functional importance. However, there have been no residue sites defined as significant with respect to either metric.

\section{Essential sites of human EGFR and GCK proteins}

To evaluate the $C M F$-significant residue sites, we have investigated essential sites of human EGFR (pdb entry 
2J6M) and GCK (pdb entry 1V4S) proteins. The essential sites of both proteins have been assigned into three main categories: i) the nsSNP positions and their adjacent sites; ii) residue positions which are located at or near active sites, allosteric sites, or binding sites; iii) residue positions which are nearby strictly conserved sites. Here, we have used "nearby" definition of Nussinov et al. [39] and defined two residues as in contact or adjacent when the distance between their major carbon atoms is less than $6 \AA$. We have defined positions which are nearby nsSNPs as essential, because several of them are also adjacent to active sites, allosteric sites, binding sites, or strictly conserved sites. Thus, we refer to a $C M F$-significant residue site as "functionally or structurally important" if it falls into one of these categories of essential sites.

\section{Position analysis of the Human Epidermal Growth Factor Receptor (EGFR) protein}

The epidermal growth factor receptor (EGFR) is a member of the ErbB (Erythroblastic Leukemia Viral Oncogene
Homolog) family receptors. Signaling through this receptor is a highly conserved mechanism from nematode to humans involved in numerous processes, including proliferation, cell fate determination, and tissue specification [40]. Furthermore, several studies have implicated that mutations resulting in misregulation of the activity or action of EGFR led to multiple cancers, including those of the brain, lung, mammary gland, and ovary [24-27]. Here, in order to detect essential mutation positions in corresponding sequence of human EGFR protein, we determined altogether $43 C M F$-significant residue sites (see Additional file 1). 15 of these significant residue sites have been verified as nsSNP sites through the Ensembl database annotation and they are illustrated in Figure 1.

Additionally, the significant sites E746, Q791, and four of the nsSNP positions (I759,Y764,M766 and K846) are also in contact with critical active site regions for gefitinib binding site in the wild type EGFR kinase [25,28] (see Figure 2).

Moreover, we observed that 17 further $C M F$-significant positions are essential sites (see Table 1). In total, we

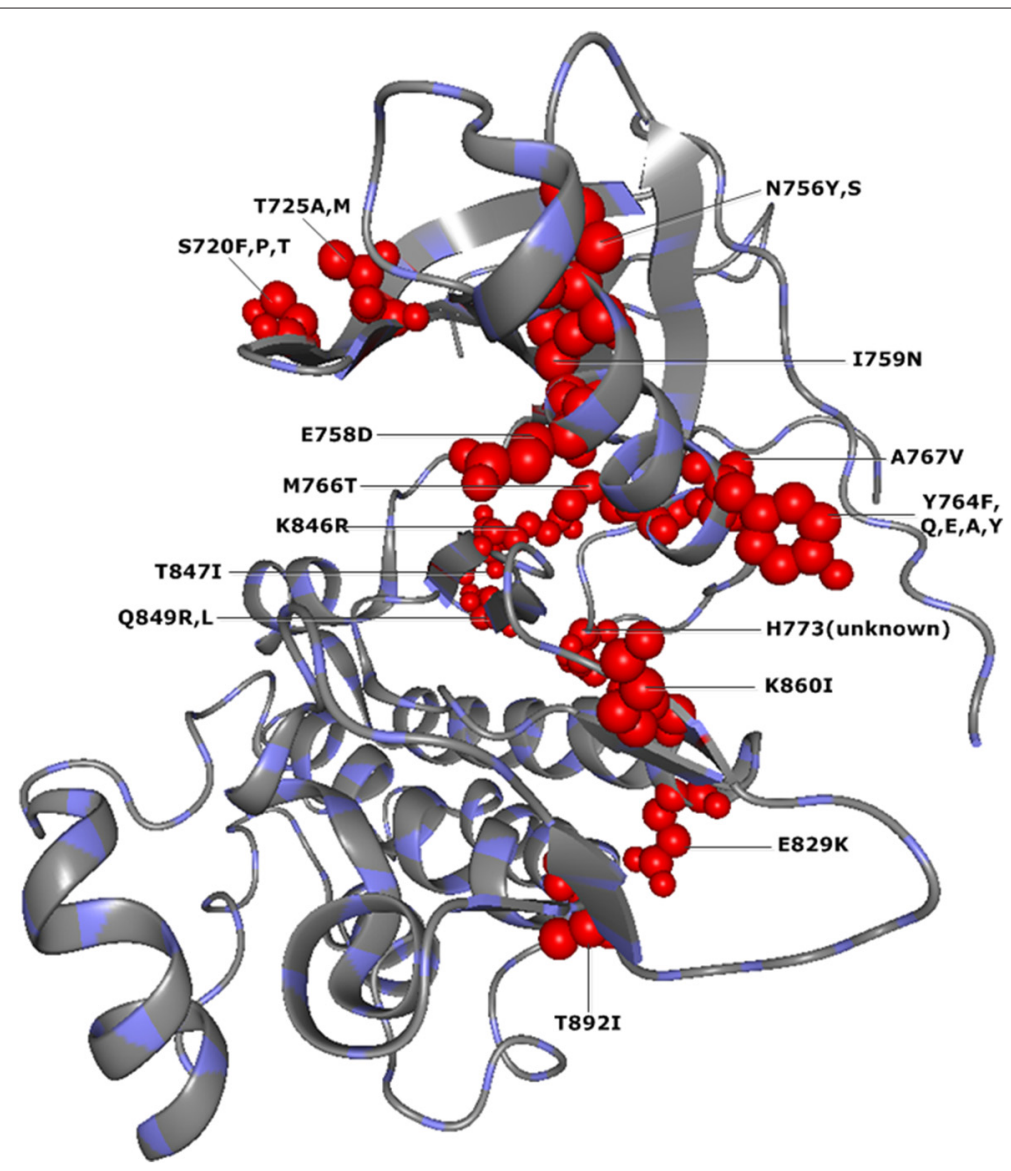

Figure 1 CMF-significant nsSNP positions in human EGFR protein (PDB-Entry 2J6M). The red spheres correspond to structural localization of 15 different nsSNP positions found by CMF as significant in the EGFR protein. 


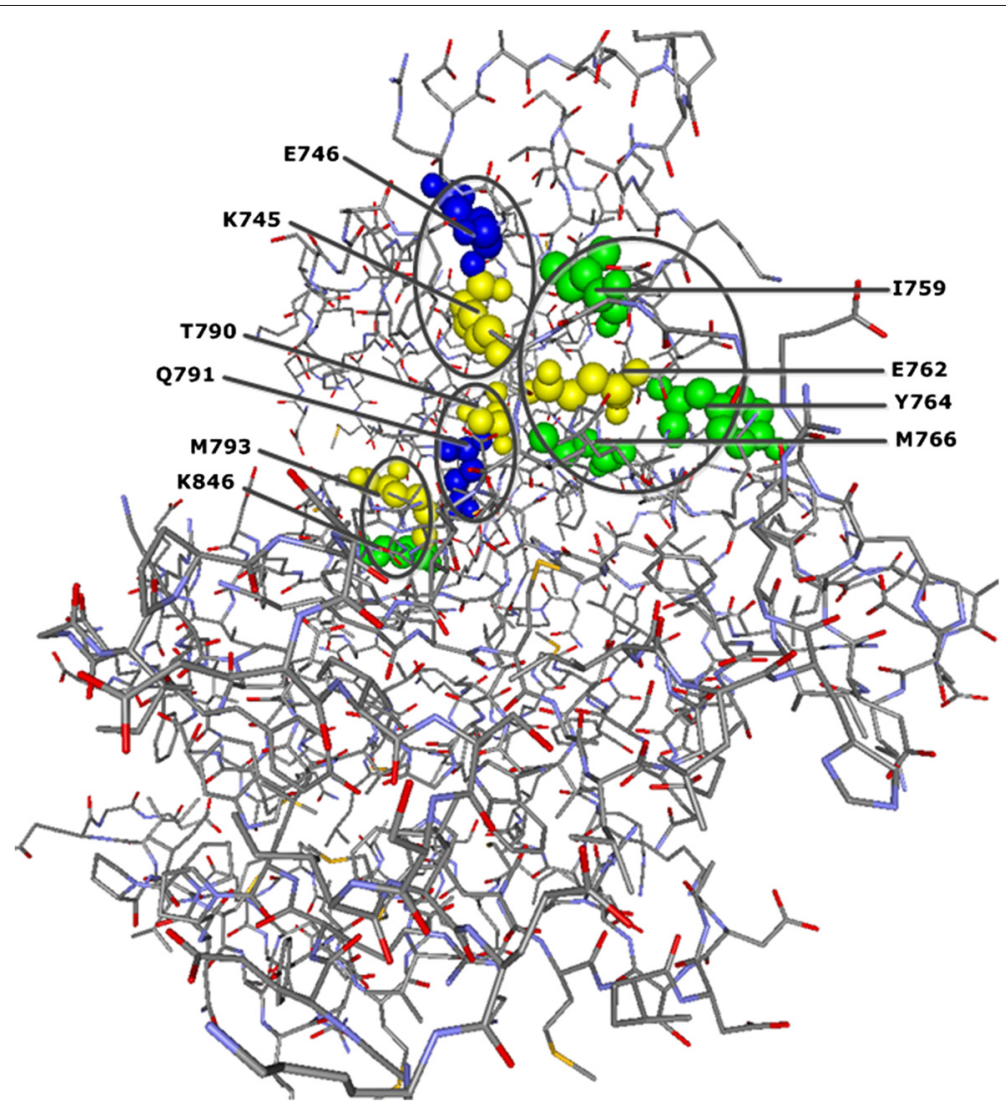

Figure $\mathbf{2}$ CMF-significant residue positions are in contact with gefitinib binding sites in human EGFR protein (PDB-Entry 2J6M ). Yellow spheres denote positions of the gefitinib binding sites in the wild type kinase. Blue spheres show the localization of significant adjacent residue positions found by CMF which are in contact with these binding sites. Moreover, the CMF-significant sites 1759, Y764, M766 and K846, shown with green spheres, are already described as nsSNP positions and they are also in contact with gefitinib binding sites E762 and M793, respectively. The circles indicate clusters of gefitinib binding sites and their significant adjacent sites.

have established here for EGFR protein the importance of 34 out of $43 C M F$-significant residue sites via different resources $[25,28,35]$.

Although the vast majority of $C M F$-significant sites are verified to be structurally or functionally important in human EGFR protein, nine CMF-significant sites do not overlap with essential sites. The reason for the high connectivity degree of these unconfirmed significant sites and their role in the EGFR protein is unclear.

\section{Position analysis of the Human Glucokinase (GCK) protein}

Glucokinase (GCK) is a monomeric enzyme catalyzing phosphorylation of glucose to glucose-6-phosphate, which is the first step in the utilization of glucose, at physiological glucose concentration in pancreas and liver. Given the fact that GCK displays low affinity for glucose, it acts as a glucose sensor playing an important role in the regulation of carbohydrate metabolism. Mutations of the GCK gene can lead to maturity onset diabetes of the young (MODY) characterized by an autosomal dominant mode of inheritance and onset early adulthood
[32], or familial hyperinsulinemic hypoglycemia type 3 (HHF), common cause of persistent hypoglycemia in infancy [41].

Applying our method, we found 72 CMF-significant sites to be structurally or functionally important in human GCK protein (see Additional file 2). 16 of these significant residue positions are related to disease associated nsSNP positions [29-31,34,35] (see Figure 3).

Furthermore, nine significant sites are found to be in contact with allosteric sites in the GCK protein structure. Among these sites, the R63 is also allosteric site by itself [32] and T209, C213 and E221 overlap with nsSNP regions (see Figure 4B). Moreover, the five significant sites T149, F171, T206, Q287, and G294 interact with glucose binding sites K169, D204, N205, and E290 [32] (see Figure 4A).

Besides this, there are further $30 C M F$-significant essential sites which are nearby nsSNPs or strictly conserved residue positions (see Table 2). Altogether, we showed the functionality of 57 positions out of $72 \mathrm{CMF}$-significant residue sites via different resources [29-35]. 
Page 5 of 12

\begin{tabular}{lll}
$\begin{array}{l}\text { Table } 1 \text { CMF-significant essential sites in human EGFR } \\
\text { protein, which are nearby either nsSNPs or strictly } \\
\text { conserved sites }\end{array}$ & Reference \\
\hline $\begin{array}{l}\text { CMF-significant } \\
\text { essential sites }\end{array}$ & $\begin{array}{l}\text { Nearby nsSNPs, or strictly } \\
\text { conserved sites }\end{array}$ & - \\
\hline Y727 & $726^{\mathbf{c}} 743^{\mathbf{c}}$ & {$[35]$} \\
H755 & $756^{\mathbf{s}}, 758^{\mathbf{s}}$ & - \\
D800 & $798^{\mathbf{c}}$ & {$[35]$} \\
G824 & $773^{\mathbf{s}}$ & {$[35]$} \\
D830 & $829^{\mathbf{s}}$ & {$[35]$} \\
E868 & $892^{\mathbf{s}}$ & {$[34]$} \\
E872 & $873^{\mathbf{s}}$ & - \\
V876 & $877^{\mathbf{c}}$ & - \\
K879 & $877^{\mathbf{c}}, 880^{\mathbf{c}}$ \\
Y891 & $892^{\mathbf{s}}, 895^{\mathbf{c}}$ \\
S899 & $880^{\mathbf{c}}, 896^{\mathbf{c}}, 898^{\mathbf{c}}, 901^{\mathbf{c}}$ \\
Y900 & $898^{\mathbf{c}}, 901^{\mathbf{c}}$ \\
T909 & $906^{\mathbf{c}}, 936^{\mathbf{c}}$ \\
S912 & $906^{\mathbf{c}}, 936^{\mathbf{c}}$ & - \\
K913 & $914^{\mathbf{c}}$ & - \\
D916 & $914^{\mathbf{c}}$ & - \\
M947 & $901^{\mathbf{c}}, 950^{\mathbf{c}}$ & - \\
\hline
\end{tabular}

s: non-synonymous snp site, ${ }^{c}$ : strictly conserved site.

While we are able to establish the large number of $C M F$ significant sites as structurally or functionally important in human GCK protein, 15 CMF-significant sites do not overlap with essential sites. Their importance in the GCK protein and the reason of high connectivity degree of these unconfirmed significant sites has not been explicitly determined yet.

\section{A comparison between $\mathbb{U}$-metric and $\mathbb{U}_{D(\alpha)}$-metric}

Similarities in physical or biochemical properties of amino acids are likely to be crucial for the detection of functionally or structurally important positions of a protein. In contrast to the $\mathbb{U}$-metric, which is a normalized mutual information that uses only the frequencies of occurrences of amino acids in the MSA columns, the novel $\mathbb{U}_{D(\alpha)}$-metric includes dissimilarities according to the BLOSUM62 matrix when calculating normalized mutual information. As a result the positions which have undergone dissimilar compensatory mutations are upscaled.

Having applied the $\mathbb{U}$-metric as well as the $\mathbb{U}_{D(\alpha)}$-metric to human EGFR and GCK proteins, the $\mathbb{U}_{D(\alpha)}$-metric has shown better sensitivity and specificity. However, only when we use the both metrics together, the sensitivity is significantly increased, whereas the specificity is only moderately decreased. The details are presented in Table 3.
It is important to note that the two metrics complement each other. Thus, we propose to use them together.

\section{CMF as a Web service}

We have implemented a $C M F$ Web service (http://cmf. bioinf.med.uni-goettingen.de) that takes an MSA in multiple FASTA format and a real number from $(0,1)$ interpreted as false discovery rate as input. It reports the results via email.

\section{Discussion}

To predict sites of structural or functional importance, we combine the known $\mathbb{U}$-metric of normalized mutual information [16] with a novel metric $\mathbb{U}_{D^{(i)}(1)}$ to enhance the influence of dissimilar compensatory mutations when measuring covariation of two sites. We discuss how we devised $\mathbb{U}_{D(1)}$ in Methods section.

To learn the frequency of compensatory mutations, we took $\mathbb{U}$-significant site pairs as training data. We did that for reasons of computation time regardless of the fact that these data are biased. To deal with this bias, one could carry through the training in an iterative process, with our training being the first iteration. For $i>0$, in the $(i+1)$-th iteration of this modified training, a doubly stochastic matrix $D_{\text {CompMut }}^{(i+1)}$ is calculated based on $\mathbb{U}_{D^{(i)}(1)}$-significant site pairs. This is done until the training data are stable.

According to Birkhoff's Theorem [43], every doubly stochastic matrix is a convex combination of permutation matrices. Moreover, from the Hardy-Littlewood-Pólya majorization theorem [44] follows that transforming the probability mass function by a doubly stochastic matrix increases entropy. Consequently, by linearly transforming the empirical amino acid pair distribution of a site pair by $D(1)$ before calculating the $\mathbb{U}$-value, we penalized those site pairs whose original distribution does not match the frequency pattern of formal dissimilar compensatory mutations in the training data described in the Methods section.

The challenge was to separate the signal caused by structural and functional constraints from the background. To address this issue, we studied only metrics $\mu$ that satisfy the following condition. The larger the $\mu(k, l)$-value, the larger the probability that the two sites $k$ and $l$ have co-evolved. Our critical assumptions were: i) the $\mu(k, l)$ values follow three different distributions, one for the signal, one for the noise, and one for pairs of completely unrelated sites; ii) there is an MSA-dependent threshold below which the metric $\mu$ does not fall with overwhelming probability, when it is applied to the site pairs of functional or structural importance to which $\mu$ is sensitive; iii) there is an MSA-dependent threshold significantly smaller then the one in (ii) such that with overwhelming probability 


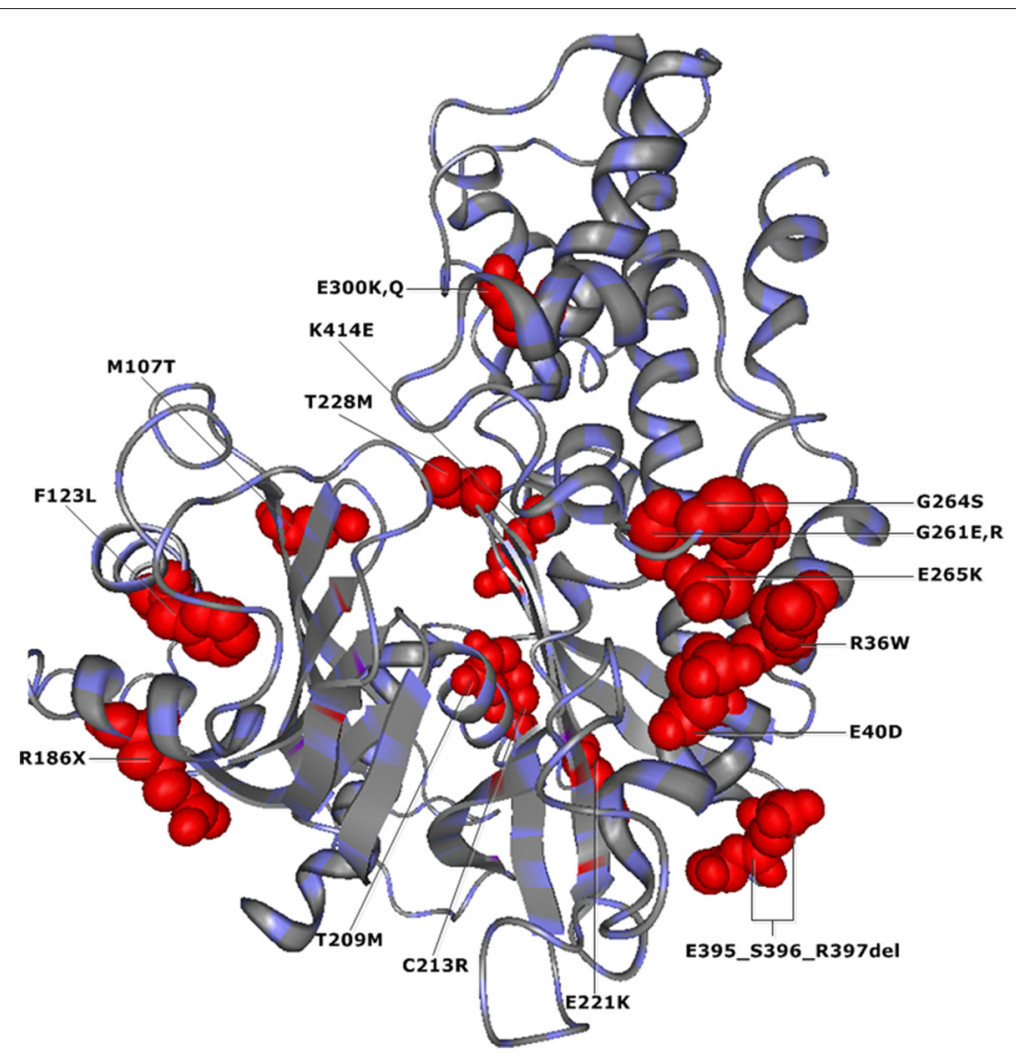

Figure 3 CMF-significant nsSNP positions in human GCK protein (PDB-Entry 1V4S). Red spheres show the structural localization of 16 different nsSNP positions found by CMF as significant in the GCK protein.
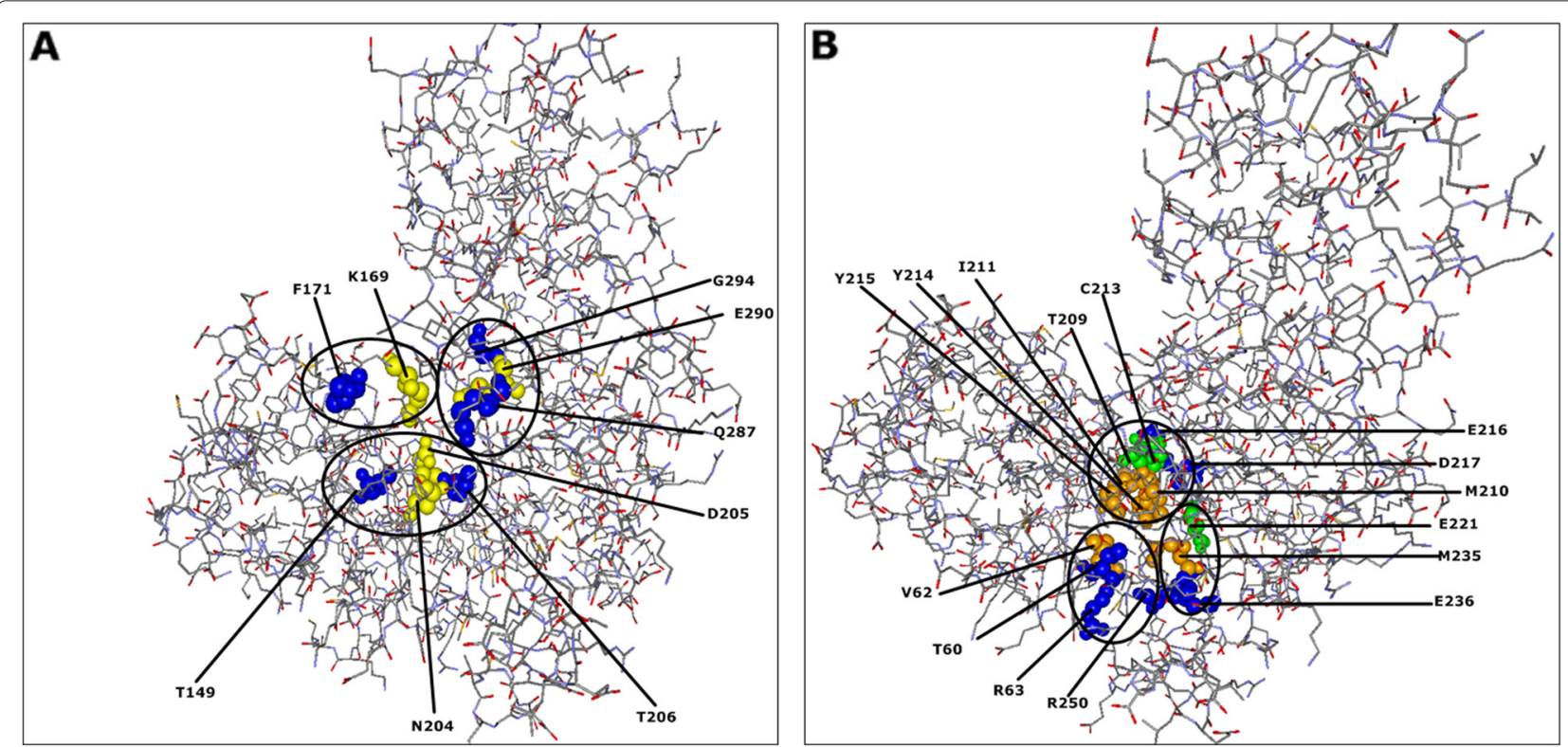

Figure $4 \mathrm{CMF}$-significant residue positions are in contact with glucose binding site and allosteric site in human GCK protein (PDB-Entry 1V4S). (A) Yellow spheres show the structural positions of the glucose binding sites (active sites). Blue spheres correspond to localization of significant adjacent residue positions found by CMF which are in contact with these active sites. (B) Orange spheres denote the allosteric sites. Blue spheres correspond to localization of just significant adjacent residue positions and green spheres indicate the significant residue positions which are already described as nsSNP position and in contact with these allosteric site. Additionally, the significant position R63 is allosteric site by itself and it is also in contact with an other allosteric site. The circles indicate clusters of glucose binding sites (A), allosteric sites (B), and their significant adjacent sites 
Table 2 CMF-significant essential sites in human GCK protein, which are nearby either nsSNPs or strictly conserved sites

\begin{tabular}{|c|c|c|}
\hline $\begin{array}{l}\text { CMF-significant } \\
\text { essential sites }\end{array}$ & $\begin{array}{l}\text { Nearby nsSNPs or strictly } \\
\text { conserved sites }\end{array}$ & Reference \\
\hline M34 & $36^{\mathbf{s}}$ & {$[34]$} \\
\hline T65 & $66^{c}$ & \\
\hline E67 & $66^{\mathbf{c}}, 68^{\mathbf{c}}$ & - \\
\hline T82 & $81^{c}$ & - \\
\hline N83 & $81^{\mathbf{c}}, 108^{\mathbf{s}}, 110^{\mathbf{s}}$ & {$[34]$} \\
\hline H105 & $106^{\mathbf{s}}$ & {$[29]$} \\
\hline C129 & $131^{\mathbf{s}}, 132^{\mathbf{s}}$ & {$[29,34]$} \\
\hline F133 & $131^{\mathbf{s}}, 132^{\mathbf{s}}$ & {$[29,34]$} \\
\hline$F 148$ & $147^{\mathbf{c}}, 150^{\mathbf{c}, \mathbf{s}}$ & {$[34]$} \\
\hline$F 152$ & $150^{\mathbf{c}, \mathbf{s}}, 151^{\mathbf{c}}$ & {$[34]$} \\
\hline H156 & $162^{\mathrm{s}}$ & {$[29]$} \\
\hline$N 180$ & $162^{\mathbf{s}}, 182^{\mathbf{s}}$ & {$[29,34]$} \\
\hline$F 260$ & $257^{\mathbf{s}}, 258^{\mathbf{c}}, 259^{\mathbf{s}}, 261^{\mathbf{s}}$ & {$[34]$} \\
\hline D262 & $259^{\mathbf{s}}, 261^{\mathbf{s}}, 264^{\mathbf{s}}$ & {$[34,42]$} \\
\hline$L 266$ & $261^{s}, 264^{s}, 265^{s}$ & {$[29,34,42]$} \\
\hline D267 & $264^{\mathbf{s}}, 265^{\mathbf{s}}$ & {$[29,42]$} \\
\hline$L 271$ & $274^{\mathrm{c}}$ & - \\
\hline$S 281$ & $278^{\mathbf{c}}, 279^{\mathbf{s}}$ & {$[34]$} \\
\hline Q286 & $259^{s}$ & {$[34]$} \\
\hline E331 & $299^{\mathbf{c}, \mathbf{s}}$ & {$[34]$} \\
\hline T332 & $295^{\mathbf{c}}, 299^{\mathbf{c}, \mathbf{s}}$ & {$[34]$} \\
\hline R333 & $336^{\mathbf{s}}$ & {$[34]$} \\
\hline Q337 & $336^{\mathbf{s}}$ & {$[34]$} \\
\hline E339 & $336^{\mathbf{s}}$ & {$[34]$} \\
\hline N391 & $392^{\mathbf{s}}$ & {$[29,34]$} \\
\hline S411 & $227^{\mathbf{c}, \mathbf{s}}, 410^{\mathbf{c}}, 414^{\mathbf{s}}$ & {$[34]$} \\
\hline$\$ 418$ & $416^{\mathbf{s}}$ & {$[30]$} \\
\hline F419 & $416^{\mathbf{s}}$ & {$[30]$} \\
\hline$E 442$ & $444^{c}$ & - \\
\hline$E 443$ & $444^{\mathrm{c}}, 445^{\mathbf{c}}$ & - \\
\hline
\end{tabular}

$\mathrm{s}_{\text {: }}$ non-synonymous snp site, ${ }^{\mathrm{c}}$ : strictly conserved site.

there are no $\mu(k, l)$-values of pairs $(k, l)$ of unrelated sites exceeding it.

In order to near-completely eliminate the noise, we filtered both our training and input data. We calculated the significant pairs such that the preassigned false discovery rate was guaranteed by generalizing the Storey-Tibshirani procedure devised for multiple testing problems [22].

Our method to eliminate noise is orthogonal to the technique developed in [19]. Therein, for every pair of sites the so-called average product correction (APC) is calculated as an explicit noise measure, by which the mutual information is then decreased. Furthermore, it generalizes the way Merkl and Zwick [16] as well as Gao et al. [17] cope with noise. According to our judgment, taking only the top 75 high-scoring pairs or the top 25 pairs into account as done in [16,17], respectively, is too conservative.

We based our noise separation technique on rather weak distribution assumptions that are standard practice in multiple hypothesis testing, instead of explicitly model the noise in terms of a metric. We applied the connectivity degree technique due to Merkl and Zwick [16] to significant site pairs with respect to our metrics. The cut-off for the connectivity degree was set to the 90-th percentile. That way we defined significant sites. Finally, a site was defined to be $C M F$-significant, if it was $\mu$-significant, where $\mu$ is either $\mathbb{U}$ or $\mathbb{U}_{D(1)}$.

Why did we set the cut-off value for the connectivity degree to the 90 -th percentile? Going through all possible $n$-th percentiles for $n=80,81, \ldots, 99$, the Matthews correlation coefficient (MCC) of a joint prediction for human EGFR and GCK proteins is maximal if $n=90$.

It is plausible that the number of functionally or structurally important sites does not only depend on the length of the protein. Therefore, the 90-th percentile cut-off should be replaced by an MSA-dependent threshold in future studies.

Our results for human EGFR and GCK proteins suggest that the large majority of significant compensatory mutation sites found by $C M F$ are in agreement with previous experimental studies regarding the functions and stability of these proteins. 15 and $16 C M F$-significant sites in human EGRF and GCK proteins, respectively, are verified as disease associated nsSNP positions (see Figures 1 and 2) where most amino acid substitutions in protein sequences damage structural stability of proteins $[36,37,45]$. Moreover, we have observed that in both proteins some of $C M F$-significant nsSNP positions are nearby allosteric sites, binding sites or catalytic sites each of which are considered to be functionally important $[46,47]$ (see Figures 2 and 4). Disease associated mutations at these nearby positions are likely to affect protein function $[38,48]$.

Despite the large number of $C M F$-significant sites demonstrated to be structurally or functionally important for both of the proteins, 9 and 15 significant sites in human EGFR and GCK proteins, respectively, are not included in essential sites. However, we hypothesize that most of the novel significant sites may play a critical role in both proteins notwithstanding the absence of previous

Table 3 Comparison between $\mathbb{U}$-metric and $\mathbb{U}_{D(\alpha)}$-metric

\begin{tabular}{lcc}
\hline & Sensitivity & Specificity \\
\hline $\mathbb{U}$-significance & $9.7 \%$ & $91.5 \%$ \\
$\mathbb{U}_{D(\alpha)}$-significance & $12.4 \%$ & $97.2 \%$ \\
$C M F$-significance & $22.1 \%$ & $88.7 \%$ \\
\hline
\end{tabular}


experimental data. Therefore, further progress from the molecular and structural biology end is required not only to assess the importance of these sites, but also for a future perspective on a deeper understanding of protein structure.

Because we have also used the $\mathbb{U}$-metric, we compared our tool with $\mathrm{H} 2 \mathrm{r}$ presented in [16] rather than with those methods developed in [17]. This way, we studied the impact of applying the Storey-Tibshirani procedure in combination with the effect of using the 90-th percentile cut-off for the connectivity degree. We have applied H2r without adding pseudo counts to the human EGFR and GCK protein. For EGFR, the 14 sites T725, A755, N756, A767, Q791, V802, N816, V819, K846, V876, M881, K913, D916, and E931 are identified as significant. Out of these significant sites, ten of these residue sites T725, A755, N756, A767, Q791,K846, V876, M881, K913, and D916 are essential sites. On the other hand, for GCK, H2r identified the 15 residue positions L25, R36, R63, M107, C213, V226, G261, D262, G264, L266, D267, E268, T405, K414, and H416 as significant. Twelve of these sites, namely R36, R63, M107, C213, V226, G261,D262, G264, L266, D267, $\mathrm{K} 414$, and H416, are essential sites. However, when using the H2r Web service (http://www-bioinf.uni-regensburg. $\mathrm{de} /$ ) to analyze EGFR and GCK proteins, sensitivity is decreased, while precision is increased. By this service only eight sites for EGFR and nine sites for GCK were found to be significant. Moreover, only five and eight of them are verified as functionally or structurally important for EGFR and GCK proteins, respectively. This difference stems from the fact that the H2r Web service tightens the filtering of the columns. In addition to this, statistically evaluating H2r for EGFR and GCK proteins, we observed a sensitivity of $5.4 \%$, specificity of $96.7 \%$, precision of $75.9 \%$, and a Matthews correlation coefficient value of 0.047 . On the other hand, the CMF reaches precision of $79.1 \%$, and a Matthews correlation coefficiant value of 0.133 . For sensitivity and specificity of the $C M F$ refer to the last row of Table 3.

The results of this comparison show that a vast majority of functionally or structurally important residue positions cannot be detected without using our novel MSA specific model and both metrics $\left(\mathbb{U}\right.$ and $\left.\mathbb{U}_{D(1)}\right)$ together.

\section{Conclusions}

The $C M F$ is a new method which includes an MSAspecific statistical model based on multiple testing procedures that quantifies the error made in terms of the false discovery rate and a novel entropy-based metric to upscale BLOSUM62 dissimilar compensatory mutations. Hence, it shows how dissimilar compensatory mutations have affected genomic sequences in the course of evolution. The method is able to predict significant compensatory mutation positions in protein sequences. We suggest that CMF could be used as a novel automated function prediction tool that is required for a better understanding of the structural basis of proteins.

\section{Methods}

In this section we describe the training data used and the methods applied and partly developed. Our descriptions follows the structure of Figure 5, i.e. we start with the data

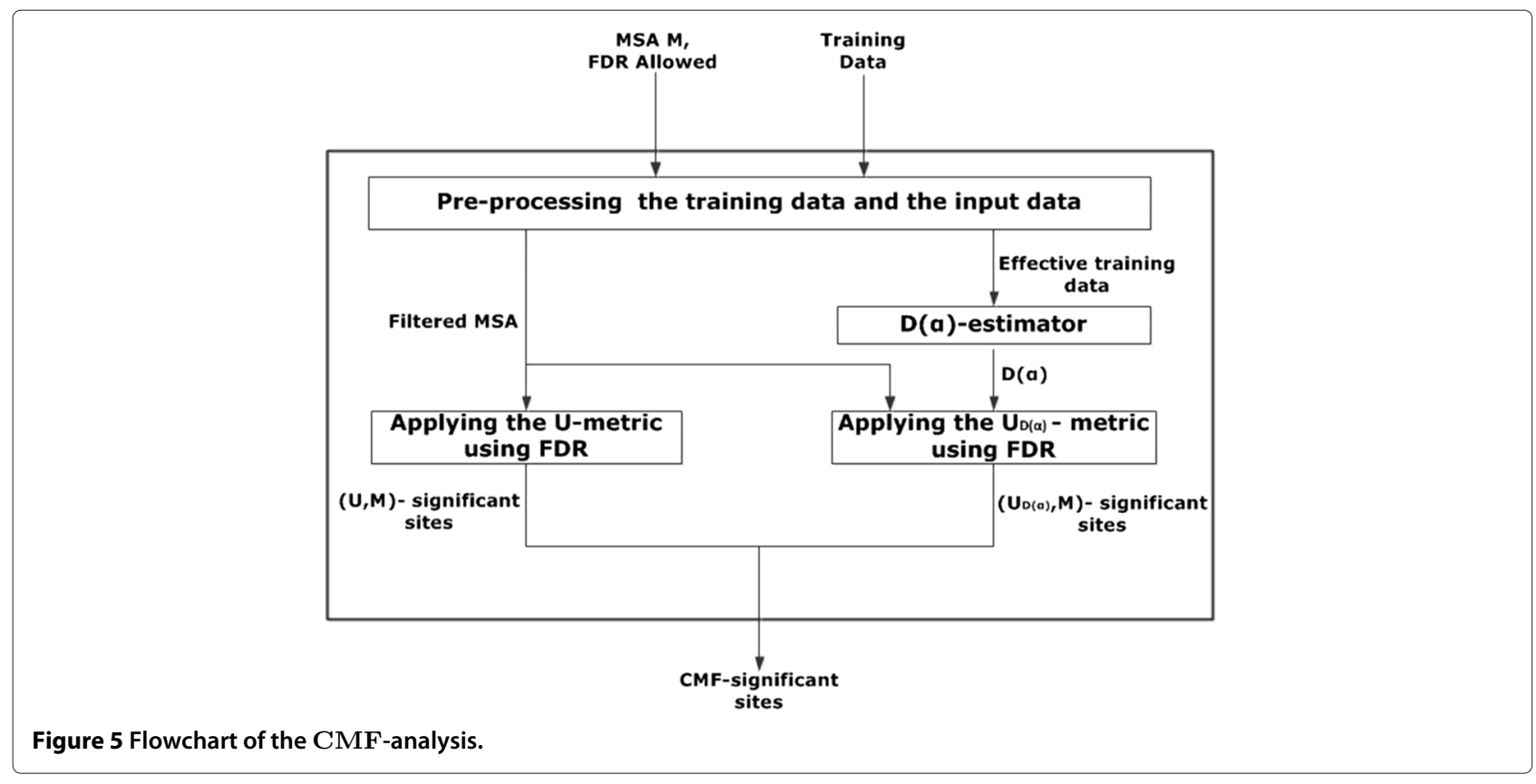


and the preprocessing and systemically work towards the $C M F$-significant site prediction.

\section{Training data set and pre-processing}

We used a redundancy free set of more than 35000 protein structures computed in Rainer Merkl's Lab at the University of Regensburg in the following way. The protein structures were taken from the protein data base (http://www.pdb.org/). The PISCES services [49] was applied to assess proteins on sequence similarity and equality of 3D-data. The related MSAs were gathered from the HSSP data base (http://swift.cmbi.ru.nl/gv/ hssp/).

Taking pattern from [16], we filtered every MSA obtained as follows. First, highly similar and dissimilar sequences were deleted to ensure that the sequence identity between any two sequences is at least $20 \%$ and no more than $90 \%$. Second, we removed strictly conserved residue columns, where the percentage of identical residues is greater than 95\%. Third, we eliminated the residue columns which contain more than $25 \%$ gaps. Finally, we discarded all MSAs with less than 125 sequences. More than 17000 MSAs survived the last filtering step. We used approximately 1700 MSAs as training data which we randomly chose from this set. The pdb entries of the corresponding protein structures are listed in Additional file 3.

\section{Detecting compensatory mutations by the $\mathbb{U}$-metric}

In [16] a normalized measure of mutual information ranging over the interval $[0,1]$ is successfully used to detect important residues. It is defined as

$$
\mathbb{U}(i, j):=2 \cdot \frac{\mathbb{H}(i)+\mathbb{H}(j)-\mathbb{H}(i, j)}{\mathbb{H}(i)+\mathbb{H}(j)},
$$

where $\mathbb{H}(i)$ and $\mathbb{H}(j)$ are the entropy of the empirical amino acid distributions of the columns $i$ and $j$, and $\mathbb{H}(i, j)$ is their joint entropy.

We determine an MSA-dependent threshold $\tau$ above which $\mathbb{U}$-values are defined as significant. Let $M$ be the MSA for the protein under investigation. We extend a standard approach of multiple testing theory $[22,50,51]$ with the following assumptions in mind. $M$ 's $\mathbb{U}(k, l)$ values follow three different distributions. The null distribution $F_{0}$ represents background signals. The distributions $G_{1}$ and $G_{2}$ model the unrelated pairs and the signal pairs, respectively.

We assume $F_{0}$ to be a $\beta$-distribution, and $M$ 's $\mathbb{U}(k, l)$ values $U_{1}, U_{2}, \ldots, U_{\mu}$ to be an independent and identically distributed (iid) sample.

Let $X_{\iota}:=1-F_{0}\left(U_{\iota}\right)$ be the $p$-value of $U_{\iota}$ with respect to $F_{0}$. If $U_{\iota}$ is $F_{0}$-distributed, then $X_{\iota}$ is uniform over [0,1]. However, if $U_{\iota}$ is $G_{1}$-distributed or $G_{2}$-distributed, then $X_{\iota}$ is skewed to 1 or to 0 (see Figure 6). According to
[22,23], the fraction $\gamma$ of the $U_{l}$ 's that are $F_{0}$-distributed is estimated by

$$
\hat{\gamma}:=\frac{\text { number of } p \text {-values in }\left[\lambda_{1}, \lambda_{2}\right]}{\mu\left(\lambda_{2}-\lambda_{1}\right)} .
$$

The tuning parameters $\lambda_{1}$ and $\lambda_{2}$ are chosen such that the fraction of not uniformly distributed $p$-values that fall into $\left[\lambda_{1}, \lambda_{2}\right]$ is negligible.

We call a pair of sites $(i, j)$ of the protein under study $(\mathbb{U}, M)$-significant if and only if the $p$-value $1-F_{0}(\mathbb{U}(i, j))$ is less than or equal to $\tau$, for a threshold $\tau \leq \lambda_{1}$ that ensures the input false discovery rate $F D R$, which in turn can be estimated by

$$
\widehat{F D R}(\tau)=\frac{\hat{\gamma} \mu \tau}{\text { number of } p \text {-values } \leq \tau} .
$$

In order to determine the parameters of the $\beta$ distribution $F_{0}$, it is sufficient to estimate the expected value and the variance. The expected value is estimated by the sample mean of all $\mathbb{U}$-values of $M$. As for the variance, we take pattern from [52]. Having drawn an iid sample $\left(C_{1}, C_{1}^{\prime}\right),\left(C_{2}, C_{2}^{\prime}\right), \ldots,\left(C_{v}, C_{v}^{\prime}\right)$ of random column pairs of a sufficient size whose $\mathbb{U}$-values fall in a preassigned subinterval of $[0,1]$, we calculate $D_{1}, D_{2}, \ldots, D_{v}$ by randomly shuffling $C_{\iota}^{\prime}$ for every $\iota=1,2, \ldots, v$. The variance is then estimated as the sample variance of $\left(C_{1}, D_{1}\right),\left(C_{2}, D_{2}\right), \ldots,\left(C_{v}, D_{v}\right)$.

The connectivity degree of a site $i$ with respect to the metric $\mathbb{U}$ and the MSA $M$ is defined as number of sites $j$ such that $(i, j)$ is $(\mathbb{U}, M)$-significant [16]. Site $i$ is defined to be $(\mathbb{U}, M)$-significant, if $i$ s connectivity degree with respect to $\mathbb{U}$ and $M$ is greater than or equal to the 90 -th percentile. The $(\mathbb{U}, M)$-significant sites of a protein do not coincide with those predicted by H2r [16]. The connectivity degrees attained and the threshold used substantially differ. In particular, the latter one is data-dependent rather than constant.

\section{Enhancing prediction by the $\mathbb{U}_{D(\alpha)}$-metric that models dissimilar compensatory mutations}

A pair $\left(\left(a_{i}, a_{j}\right),\left(a_{k}, a_{l}\right)\right)$ of amino acid pairs is defined to be a formal dissimilar compensatory mutation, if the BLOSUM62 score both of $\left(a_{i}, a_{k}\right)$ and $\left(a_{j}, a_{l}\right)$ is negative.

We use the training data set of approximately 1700 MSAs described above to estimate a $400 \times 400$ doubly stochastic matrix $D_{\text {CompMut }}$ This matrix is our mathematical model of how dissimilar compensatory mutations have affected genomic sequences in the course of evolution. Its training consists of five phases.

Phase 1. We calculate a signal and a null set of column pairs. The signal set consists of all $(\mathbb{U}, M)$-significant column pairs, where $M$ ranges over all training MSA. The null set consists of sufficiently many column pairs 




randomly chosen from every training MSA. For both the signal set and the null set we compute a symmetric $400 \times 400$ integer-valued matrix of frequencies of pair substitutions $C_{\text {alt }}$ and $C_{\text {null }}$. To this end, the method used to compute BLOSUM62 matrices [53] is applied to count residue pair substitutions in MSA column pairs rather than residue substitution in columns.

Phase 2. Using $C_{\text {alt }}$ and $C_{\text {null, }}$, we define the matrix $C_{\text {sig }}$ by

$$
\begin{aligned}
& C_{\text {sig }}\left(\left(a_{i}, a_{j}\right),\left(a_{k}, a_{l}\right)\right) \\
& \quad:= \begin{cases}C_{\text {alt }}\left(\left(a_{i}, a_{j}\right),\left(a_{k}, a_{l}\right)\right) & \text { if } \varphi\left(\left(a_{i}, a_{j}\right),\left(a_{k}, a_{l}\right)\right)=1 ; \\
0 & \text { otherwise }\end{cases}
\end{aligned}
$$

where $\varphi\left(\left(a_{i}, a_{j}\right),\left(a_{k}, a_{l}\right)\right)=1$ if and only if $\left(a_{i}, a_{j}\right)=$ $\left(a_{k}, a_{l}\right)$ or

$$
\begin{gathered}
\frac{C_{\text {alt }}\left(\left(a_{i}, a_{j}\right),\left(a_{k}, a_{l}\right)\right)}{\sum_{i^{\prime}, j^{\prime}, k^{\prime}, l^{\prime}} C_{\text {alt }}\left(\left(a_{i^{\prime}}, a_{j^{\prime}}\right),\left(a_{k^{\prime}}, a_{l^{\prime}}\right)\right)} \\
>\frac{C_{\text {null }}\left(\left(a_{i}, a_{j}\right),\left(a_{k}, a_{l}\right)\right)}{\sum_{i^{\prime}, j^{\prime}, k^{\prime}, l^{\prime}} C_{\text {null }}\left(\left(a_{i^{\prime}}, a_{j^{\prime}}\right),\left(a_{k^{\prime}}, a_{l^{\prime}}\right)\right)} .
\end{gathered}
$$

Phase 3. We set all entries of the matrix $C_{\text {sig }}$ outside the main diagonal that do not represent a formal dissimilar compensatory mutation to zero. This results in the matrix $C_{\text {CompMut }}$. By normalizing $C_{\text {CompMut }}$, we obtain a symmetric matrix $P_{\text {CompMut }}$. For $a_{i}, a_{j}, a_{k}, a_{l}$ ranging over all amino acids, $P_{\text {CompMut }}\left(\left(a_{i}, a_{j}\right),\left(a_{k}, a_{l}\right)\right)$ represents an empirical probability distribution on pairs of amino acid pairs.
Phase 4. We calculate the symmetric $400 \times 400$-matrix

$S_{\text {CompMut }}:=\left(\log \frac{P_{\text {CompMut }}\left(\left(a_{i}, a_{j}\right),\left(a_{k}, a_{l}\right)\right)}{P_{\text {CompMut }}^{\mathrm{b}}\left(a_{i}, a_{j}\right) P_{\text {CompMut }}^{\mathrm{b}}\left(a_{k}, a_{l}\right)}\right)_{\left(a_{i}, a_{j}\right),\left(a_{k}, a_{l}\right)}$,

where $P_{\text {CompMut }}^{\mathrm{b}}\left(a_{i}, a_{j}\right)$ is the marginal distribution of $P_{\text {CompMut. }}$

Phase 5. We set all negative entries of $S_{\text {CompMut }}$ to zero. Then we compute the doubly stochastic matrix $D_{\text {CompMut }}$ by means of the canonical iterated row-column normalization procedure [54].

Now we define our new $\mathbb{U}_{D(\alpha)}$-metric based on $D_{\text {CompMut }}$. For every column pair $(i, j)$ of the input MSA $M$, we linearly transform the associated empirical pair distribution with the doubly stochastic matrix

$$
D(\alpha):=(1-\alpha) \mathbf{1}+\alpha D_{\text {CompMut }}
$$

where 1 is the $400 \times 400$ unit matrix, $D_{\text {CompMut }}$ is the result of training phase 5 , and $\alpha \in(0,1]$ is a preassigned real number. $\mathbb{U}_{D(\alpha)}(i, j)$ is then defined to be the $\mathbb{U}$-value (see Equation 1) of this transform.

Having canonically carried over the definition of a significant site pair and of the connectivity degree of a site to this case, a site $i$ is called $\left(\mathbb{U}_{D(\alpha)}, M\right)$-significant, if $i$ 's connectivity degree with respect to the metric $\mathbb{U}_{D(\alpha)}$ is greater than or equal to the 90-th percentile.

Finally, a site is defined to be CMF-significant with respect to the MSA $M$, if it is $(\mathbb{U}, M)$-significant or $\left(\mathbb{U}_{D(\alpha)}, M\right)$-significant. The $C M F$-significant sites are predicted as functionally or structurally important ones. 
Principally, the controlling parameter $\alpha \in(0,1]$ can be adjusted by the user. We set $\alpha$ to 1 to allow the two sets of $(\mathbb{U}, M)$-significant and $\left(\mathbb{U}_{D(\alpha)}, M\right)$-significant positions to complement each other.

Note, that the matrix $S_{\text {CompMut }}$ could be replaced with another scoring matrix meaningful in this context.

\section{Additional files}

Additional file 1: EGFR significant sites. CMF-significant residue sites of the human epidermal growth factor receptor (EGFR) protein.

Additional file 2: GCK significant sites. CMF-significant residue sites of the human glucokinase (GCK) protein.

Additional file 3: Pdb entries of training MSAs. Pdb entries of redundancy free data set.

\section{Competing interests}

The authors declare that they have no competing interests.

\section{Authors' contributions}

SW developed the model underlying CMF. MG developed the model together with SW, designed and implemented the tool, and interpreted the results together with NT and $\mathrm{MH}$. All authors read and approved the manuscript.

\section{Acknowledgements}

We thank Rainer Merkl for placing a redundancy free set of more than 35000 protein structures at our disposal. Special thank goes to Torsten Schöps for setting up the CMF Web server. Mehmet Gltas acknowledges financial support by the Deutsche Forschungsgemeinschaft (DFG: WA 766/7-1).

\section{Author details}

${ }^{1}$ Institute of Computer Science, University of Göttingen, Goldschmidtstr. 7, 37077, Göttingen, Germany. ${ }^{2}$ Department of Bioinformatics, University of Göttingen, Goldschmidtstr. 1, 37077 Göttingen, Germany. ${ }^{3}$ Erasmus MC Stem Cell Institute, Department of Cell Biology, Erasmus Medical Center, Rotterdam, The Netherlands.

Received: 18 May 2012 Accepted: 23 August 2012 Published: 11 September 2012

\section{References}

1. Jeon J, Yang JS, Kim S: Integration of Evolutionary Features for the Identification of Functionally Important Residues in Major Facilitator Superfamily Transporters. PLoS Comput Bio/ 2009, 5(10):e1000522. [http://dx.doi.org/10.13712Fjournal.pcbi.1000522]

2. Sadovsky $E$, Yifrach $O$ : Principles underlying energetic coupling along an allosteric communication trajectory of a voltage-activated $\mathrm{K}+$ channel. Proc Nat Acad Sci 2007, 104(50):19813-19818. [http://www. pnas.org/content/104/50/19813.abstract]

3. Pupko T, Bell RE, Mayrose I, Glaser F, Ben-Tal N: Rate4Site: an algorithmic tool for the identification of functional regions in proteins by surface mapping of evolutionary determinants within their homologues. Bioinformatics 2002, 18(suppl 1):S71 — S77. [http:// bioinformatics.oxfordjournals.org/content/18/suppl_1/S71.abstract]

4. Wilson K, Walker J: Principles and Techniques of Biochemistry and Molecular Biology. 7th edition. New York: Cambridge University Press; 2010.

5. Altschuh D, Lesk AM, Bloomer AC, Klug A: Correlation of co-ordinated amino acid substitutions with function in viruses related to tobacco mosaic virus. $J$ Mol Biol 1987, 193(4):693-707.

6. Martin LC, Gloor GB, Dunn SD, Wahl LM: Using information theory to search for co-evolving residues in proteins. Bioinformatics 2005, 21(22):4116-4124.

7. Yeang $\mathrm{CH}$, Haussler D: Detecting Coevolution in and among Protein Domains. PLoS Comput Biol 2007, 3(11):e211. [http://dx.plos.org/10. 13712Fjournal.pcbi.0030211]

8. Goh CS, Bogan AA, Joachimiak M, Walther D, Cohen FE: Co-evolution of proteins with their interaction partners. $J \mathrm{Mol} B i \mathrm{O} / 2000$,
299(2):283-293. [http://www.sciencedirect.com/science/article/pii/ S002228360093732X]

9. Lockless SW, Ranganathan R: Evolutionarily Conserved Pathways of Energetic Connectivity in Protein Families. Science 1999, 286(5438):295-299. [http://www.sciencemag.org/content/286/5438/295. abstract]

10. Göbel U, Sander C, Schneider R, Valencia A: Correlated mutations and residue contacts in proteins. Proteins-Struct Funct Genet 1994, 18(4):309-317.

11. Neher $E$ : How frequent are correlated changes in families of protein sequences? Proc Nat AcadSci 1994, 91:98-102. [http://www.pnas.org/ content/91/1/98.abstract]

12. Pollock DD, Taylor WR: Effectiveness of correlation analysis in identifying protein residues undergoing correlated evolution. Protein Eng 1997, 10(6):647-657. [http://peds.oxfordjournals.org/content/ 10/6/647.abstract]

13. Dekker JP, Fodor A, Aldrich RW, Yellen G: A perturbation-based method for calculating explicit likelihood of evolutionary co-variance in multiple sequence alignments. Bioinformatics 2004, 20(10): 1565-1572.

14. Atchley WR, Wollenberg KR, Fitch WM, Terhalle W, Dress AW: Correlations among amino acid sites in bHLH protein domains: an information theoretic analysis. Mol Biol Evol 2000, 17:164.

15. Tillier ER, Lui TW: Using multiple interdependency to separate functional from phylogenetic correlations in protein alignments. Bioinformatics 2003, 19(6):750-755. [http://bioinformatics.oxfordjournals. org/content/19/6/750.abstract]

16. Merkl R, Zwick M: H2r: Identification of evolutionary important residues by means of an entropy based analysis of multiple sequence alignments. BMC Bioinformatics 2008, 9:151. [http://www. biomedcentral.com/1471-2105/9/151]

17. Gao H, Dou Y, Yang J, Wang J: New methods to measure residues coevolution in proteins. BMC Bioinformatics 2011, 12:206. [http://www. biomedcentral.com/1471-2105/12/206]

18. Codoner FM, Fares M: Why Should We Care About Molecular Coevolution? Evolutionary c 2008, 4:29-38.

19. Dunn SD, Wahl LM, Gloor GB: Mutual information without the influence of phylogeny or entropy dramatically improves residue contact prediction. Bioinformatics 2008, 24(3):333-340.

20. Noivirt $O$, Eisenstein $M$, Horovitz A: Detection and reduction of evolutionary noise in correlated mutation analysis. Protein Eng Design and Sel 2005, 18(5):247-253. [http://peds.oxfordjournals.org/content/18/ 5/247.abstract]

21. Wollenberg KR, Atchley WR: Separation of phylogenetic and functional associations in biological sequences by using the parametric bootstrap. Proc Nat Acad Sci 2000, 97(7):3288-3291. [http:// www.pnas.org/content/97/7/3288.abstract]

22. Storey JD, Tibshirani R: Statistical significance for genomewide studies. Proc Acad Sci 2003, 100:9440-9445.

23. Walsh B: Multiple comparisons: Bonferroni Corrections and False Discovery Rates. Lecture Notes EEB 581, Department of Ecology and Evolutionary Biology, University of Arizona 2004.

24. Dixit A, Yi L, Gowthaman R, Torkamani A, Schork NJ, Verkhivker GM: Sequence and Structure Signatures of Cancer Mutation Hotspots in Protein Kinases. PLOS ONE 2009, 4(10):e7485. [http://dx.doi.org/10. 13712Fjournal.pone.0007485]

25. Yun CH, Boggon TJ, Li Y, Woo MS, Greulich H, Meyerson M, Eck MJ: Structures of Lung Cancer-Derived EGFR Mutants and Inhibitor Complexes: Mechanism of Activation and Insights into Differential Inhibitor Sensitivity. Cancer Cell 2007, 11(3):217-227. [http://www. sciencedirect.com/science/article/pii/S1535610807000281]

26. Zhang H, Berezov A, Wang Q, Zhang G, Drebin J, Murali R, Greene MI: ErbB receptors: from oncogenes to targeted cancer therapies. $J$ Clin Invest 2007, 117(8):2051-2058. [http://www.jci.org/articles/view/32278]

27. Lynch TJ, Bell DW, Sordella R, Gurubhagavatula S, Okimoto RA, Brannigan BW, Harris PL, Haserlat SM, Supko JG, Haluska FG, Louis DN, Christiani DC, Settleman J, Haber DA: Activating Mutations in the Epidermal Growth Factor Receptor Underlying Responsiveness of Non-Small-Cell Lung Cancer to Gefitinib. New England J Med 2004, 350(21):2129-2139. [http://www.nejm.org/doi/full/10.1056/NEJMoa040938] 
28. Balius TE, Rizzo RC: Quantitative Prediction of Fold Resistance for Inhibitors of EGFR. Biochemistry 2009, 48(35):8435-8448. [PMID: 19627157]. [http://pubs.acs.org/doi/abs/10.1021/bi900729a]

29. Tinto N, Zagari A, Capuano M, De Simone A, Capobianco V, Daniele G Giugliano M, Spadaro R, Franzese A, Sacchetti L: Glucokinase Gene Mutations: Structural and Genotype-Phenotype Analyses in MODY Children from South Italy. PLOS ONE 2008, 3(4):e1870. [http://dx.plos. org/10.13712Fjournal.pone.0001870]

30. Capuano M, Garcia-Herrero CM, Tinto N, Carluccio C, Capobianco V, Coto I, Cola A, lafusco D, Franzese A, Zagari A, Navas MA, Sacchetti L: Glucokinase (GCK) Mutations and Their Characterization in MODY2 Children of Southern Italy. PLOS ONE 2012, 7(6):e38906. [http://dx.doi. org/10.13712Fjournal.pone.0038906]

31. Garcia-Herrero CM, Rubio-Cabezas O, Azriel S, Gutierrez-Nogues A, Aragones A, Vincent O, Campos-Barros A, Argente J, Navas MA: Functional Characterization of MODY2 Mutations Highlights the Importance of the Fine-Tuning of Glucokinase and Its Role in Glucose Sensing. PLOS ONE 2012, 7:e30518. [http://dx.doi.org/10. 13712Fjournal.pone.0030518]

32. Kamata K, Mitsuya M, Nishimura T, ichi Eiki J, Nagata Y: Structural Basis for Allosteric Regulation of the Monomeric Allosteric Enzyme Human Glucokinase. Structure 2004, 12(3):429-438. [http://www. sciencedirect.com/science/article/pii/S0969212604000474]

33. Hamosh A, Scott AF, Amberger JS, Bocchini CA, McKusick VA: Online Mendelian Inheritance in Man (OMIM), a knowledgebase of human genes and genetic disorders. Nucleic Acids Res 2005, 33(suppl 1):D514_D517. [http://nar.oxfordjournals.org/content/33/suppl_1/D514. abstract]

34. Reichert J, Sühnel J: The IMB Jena Image Library of Biological Macromolecules: 2002 update. Nucleic Acids Res 2002, 30:253-254 [http://nar.oxfordjournals.org/content/30/1/253.abstract]

35. Flicek P, Amode MR, Barrell D, Beal K, Brent S, Chen Y, Clapham P, Coates G, Fairley S, Fitzgerald S, Gordon L, Hendrix M, Hourlier T, Johnson N, Kähäri A Keefe D, Keenan S, Kinsella R, Kokocinski F, Kulesha E, Larsson P, Longden I, McLaren W, Overduin B, Pritchard B, Riat HS, Rios D, Ritchie GRS, Ruffier M, et al: Ensembl 2011. Nucleic Acids Res 2011, 39(suppl 1):D800-D806. [http://nar.oxfordjournals.org/content/39/suppl_1/D800.abstract]

36. Sunyaev $S$, Ramensky $V$, Bork P: Towards a structural basis of human non-synonymous single nucleotide polymorphisms. Trends in Genet 2000, 16(5):198-200. [http://www.sciencedirect.com/science/article/pii/ S0168952500019880]

37. Wang Z, Moult J: SNPs, protein structure, and disease. Human Mutation 2001, 17(4):263-270. [http://dx.doi.org/10.1002/humu.22]

38. Burke D, Worth C, Priego EM, Cheng T, Smink L, Todd J, Blundell T: Genome bioinformatic analysis of nonsynonymous SNPs. BMC Bioinformatics 2007, 8:301. [http://www.biomedcentral.com/1471-2105/ 8/301]

39. Keskin O, Tsai CJ, Wolfson $\mathrm{H}$, Nussinov R: A new, structurally nonredundant, diverse data set of protein-protein interfaces and its implications. Protein Science 2004, 13(4):1043-1055. [http://dx.doi.org/ 10.1110/ps.03484604]

40. Herbst RS: Review of epidermal growth factor receptor biology. Int J Radiat Oncol Biol Phys 2004, 59(Supplement 2):S21 - S26. [http://www. sciencedirect.com/science/article/pii/S0360301604003311]

41. Thornton PS, Satin-Smith MS, Herold K, Glaser B, Chiu KC, Nestorowicz A, Permutt M, Baker L, Stanley CA: Familial hyperinsulinism with apparent autosomal dominant inheritance: Clinical and genetic differences from the autosomal recessive variant. J Pediatrics 1998, 132:9-14. [http://www.sciencedirect.com/science/article/pii/S0022347698704779]

42. Sherry ST, Ward MH, Kholodov M, Baker J, Phan L, Smigielski EM, Sirotkin K dbSNP: the NCBI database of genetic variation. Nucleic Acids Res 2001, 29:308-311. [http://nar.oxfordjournals.org/content/29/1/308.abstract]

43. Birkhoff $\mathrm{G}$ : Tres observationes sobre et algebra lineal. Univ NaC Tucaman Rev 1946, A(5):147-151.

44. Hardy G, Littlewood J, Pólya G: Inequalities. 2nd edition. Oxford: Oxford University Press; 1952.

45. Cheng TMK, Lu YE, Vendruscolo M, Lio' P, Blundell TL: Prediction by Graph Theoretic Measures of Structural Effects in Proteins Arising from Non-Synonymous Single Nucleotide Polymorphisms. PLOS Comput Biol 2008, 4(7):e1000135. [http://dx.doi.org/10.13712Fjournal. pcbi.1000135]
46. Bao L, Cui Y: Functional impacts of non-synonymous single nucleotide polymorphisms: Selective constraint and structural environments. FEBS Letters 2006, 580(5):1231-1234. [http://www. sciencedirect.com/science/article/pii/S0014579306000755]

47. Ng PC, Henikoff S: SIFT: predicting amino acid changes that affect protein function. Nucleic Acids Res 2003, 31(13):3812-3814. [http://nar. oxfordjournals.org/content/31/13/3812.abstract]

48. Ramensky V, Bork P, Sunyaev S: Human non-synonymous SNPs: server and survey. Nucleic Acids Res 2002, 30(17):3894-3900. [http://nar oxfordjournals.org/content/30/17/3894.abstract]

49. Wang G, Dunbrack Jr RLD: PISCES: recent improvements to a PDB sequence culling server. Nucleic Acids Res 2005, 33(Web-Server-Issue): 94-98.

50. Benjamini $Y$, Hochberg $Y$ : Controlling the False Discovery Rate: $\mathbf{A}$ Practical and Powerful Approach to Multiple Testing. J R Stat Soc Ser B (Methodological) 1995, 57:289-300. [http://www.jstor.org/stable/ 2346101]

51. Ferreira JA, Zwinderman AH: On the Benjamini-Hochberg Method. Ann Stat 2006, 34(4):1827-1849. [http://www.jstor.org/stable/25463486]

52. Bremm S, Schreck T, Boba P, Held S, Hamacher K: Computing and visually analyzing mutual information in molecular co-evolution. BMC Bioinformatics 2010, 11:330. [http://www.biomedcentral.com/14712105/11/330]

53. Henikoff S, Henikoff JG: Amino acid substitution matrices from protein blocks. Proc Nat Acad Sci 1992, 89(22):10915-10919. [http:// www.pnas.org/content/89/22/10915.abstract]

54. Cappellini V, Sommer HJ, Bruzda W, Zyczkowski K: Random bistochastic matrices. J Phys A: Math Theor 2009, 42:23.

doi:10.1186/1471-2105-13-225

Cite this article as: Gültas et al:: Coupled mutation finder: A new entropybased method quantifying phylogenetic noise for the detection of compensatory mutations. BMC Bioinformatics 2012 13:225.

\section{Submit your next manuscript to BioMed Central} and take full advantage of:

- Convenient online submission

- Thorough peer review

- No space constraints or color figure charges

- Immediate publication on acceptance

- Inclusion in PubMed, CAS, Scopus and Google Scholar

- Research which is freely available for redistribution

Submit your manuscript at www.biomedcentral.com/submit
Biomed Central 
9.2 Appendix B: Quantum Coupled Mutation Finder

9.2 Appendix B: Quantum Coupled Mutation Finder 


\title{
Quantum coupled mutation finder: predicting functionally or structurally important sites in proteins using quantum Jensen-Shannon divergence and CUDA programming
}

Mehmet Gültas ${ }^{1,2^{*}}$, Güncel Düzgün ${ }^{1}$, Sebastian Herzog ${ }^{1}$, Sven Joachim Jäger ${ }^{1}$, Cornelia Meckbach ${ }^{1}$, Edgar Wingender ${ }^{2}$ and Stephan Waack ${ }^{*}$

\begin{abstract}
Background: The identification of functionally or structurally important non-conserved residue sites in protein MSAs is an important challenge for understanding the structural basis and molecular mechanism of protein functions. Despite the rich literature on compensatory mutations as well as sequence conservation analysis for the detection of those important residues, previous methods often rely on classical information-theoretic measures. However, these measures usually do not take into account dis/similarities of amino acids which are likely to be crucial for those residues. In this study, we present a new method, the Quantum Coupled Mutation Finder (QCMF) that incorporates significant dis/similar amino acid pair signals in the prediction of functionally or structurally important sites.

Results: The result of this study is twofold. First, using the essential sites of two human proteins, namely epidermal growth factor receptor (EGFR) and glucokinase (GCK), we tested the QCMF-method. The QCMF includes two metrics based on quantum Jensen-Shannon divergence to measure both sequence conservation and compensatory mutations. We found that the QCMF reaches an improved performance in identifying essential sites from MSAs of both proteins with a significantly higher Matthews correlation coefficient (MCC) value in comparison to previous methods. Second, using a data set of 153 proteins, we made a pairwise comparison between QCMF and three conventional methods. This comparison study strongly suggests that QCMF complements the conventional methods for the identification of correlated mutations in MSAs.

Conclusions: QCMF utilizes the notion of entanglement, which is a major resource of quantum information, to model significant dissimilar and similar amino acid pair signals in the detection of functionally or structurally important sites. Our results suggest that on the one hand QCMF significantly outperforms the previous method, which mainly focuses on dissimilar amino acid signals, to detect essential sites in proteins. On the other hand, it is complementary to the existing methods for the identification of correlated mutations. The method of QCMF is computationally intensive. To ensure a feasible computation time of the QCMF's algorithm, we leveraged Compute Unified Device Architecture (CUDA).

The QCMF server is freely accessible at http://qcmf.informatik.uni-goettingen.de/.
\end{abstract}

\footnotetext{
*Correspondence: gueltas@cs.uni-goettingen.de,

waack@cs.uni-goettingen.de;

Institute of Computer Science, University of Göttingen, Goldschmidtstr. 7,

37077 Göttingen, Germany

${ }^{2}$ Institute of Bioinformatics, University of Göttingen, Goldschmidtstr. 1, 37077

Göttingen, Germany
}

Biomed Central

(c) 2014 Gültas et al.; licensee BioMed Central Ltd. This is an Open Access article distributed under the terms of the Creative Commons Attribution License (http://creativecommons.org/licenses/by/2.0), which permits unrestricted use, distribution, and reproduction in any medium, provided the original work is properly credited. 


\section{Background}

Multiple sequence alignments (MSAs) of homologous protein sequences give us information about two major features of the proteins of interest. The first one consists of easily detectable highly conserved residue sites that are obviously important for the structure and/or the function of the protein; while the second one corresponds to compensatory (coupled) mutations between two or more residue sites that also contain crucial information on the structural and functional basis of proteins [1]. These compensatory mutations occur according to the functional coupling of mutation positions which might be explained as one mutation in a certain site affecting a compensating mutation at another site, even if both related residue sites are distantly positioned in the protein structure [2-5]. In particular, such mutations at essential residue sites are likely to destroy protein structure which often results in loss of the protein function [6,7]. Thus, recognition of these residue sites is as important as the strictly conserved positions for the understanding of the structural basis of protein functions and for the identification of functionally important residue positions $[5,8,9]$.

Although the strictly conserved residue sites are easily detectable and interpretable in MSAs, the detection of important non-conserved compensatory mutation sites needs more complex approaches. Today, due to the simplicity and efficiency, the mutual-information-based metrics (MI-metrics) are often used to measure the coevolutionary relationship between residue sites in MSAs [4-6,10-13]. However, the MI-metrics strongly depend on the amino acid distributions observed in the MSA columns rather than on physical or biochemical constraints of amino acids that are likely to be crucial for the detection of functionally or structurally important compensatory mutations in a protein sequence. Further, according to the phylogenetic relationship of protein sequences and background noise, there is always a MIvalue between each column pair in an MSA. Therefore, the challenging problems in bioinformatics for the detection of significant compensatory mutation signals are: i) the minimization of the influence of phylogenetic relationships of protein sequences by incorporating physical or biochemical properties of amino acids in the calculation; ii) the separation of significant signals from the background noise or unrelated pair signals.

In order to eliminate the influence of phylogeny and noise effects of MI, Dunn et al. [6] have introduced the average product correction (APC). Subtracting APC from MI, they obtained their MIp metric. However, in their model the reduction of background noise is not quantified. On the other hand, Gao et al. [13] have integrated amino acid background distribution (MIB) in the calculation of their MI-metric and focused on only 25 column pairs of each MSA with the highest normalized MI values as significant to reduce noisy effect which seems to be over-conservative, yet specific.

Large efforts have been made in the last few years to improve local-correlation-measure-based approaches to residue co-evolution when it comes to modeling effects that rely on spatial proximity (see [14] for an overview). In this case, it is necessary to disentangle direct and indirect correlations. Classical mutual information, for example, is high not only if the two sites under study are close in 3D space. Quite the contrary, any local measure of correlation, not just mutual information, is limited by the transitivity effect.

To overcome this problem, global statistical models of protein families are employed. The direct-coupling analysis (DCA) works as follows. Maximizing the entropy subject to preserving the single and pair residue frequencies observed, a joint probability distribution on all possible members of the protein family is derived. Utilizing this distribution, considerable progress in predicting residue-residue contacts in 3-dimensional protein structures was made [15-17]. Protein Sparse Inverse Covariance (PSICOV) [18] achieves disentanglement of direct and indirect correlations by inverting a residue-residue covariance matrix. In [19] further progress was made by integrating structural context and sequence co-evolution information.

There is merely a small number of methods that incorporate amino acid similarity in the prediction of functionally or structurally important sites. In this context, it is natural to partition the amino acids into chemically similar groups before applying an information-theoretic measure like the Shannon entropy $[20,21]$. It was reported that many other methods fail to outperform this simple partition approach [22]. However, quantum information theory supplies a well-studied and powerful framework to integrate such similarity, where the classical Shannon entropy is swapped for the von Neumann entropy (VNE). Caffrey et al. [23] and Johansson et al. [24] have firstly introduced VNE to multiple sequence alignment analysis although they did not treat amino acid pair similarity.

Recently, a new method called Coupled Mutation Finder (CMF) has been introduced by Gültas et al. [5] to deal with phylogenetic noise as well as background signals and to quantify the error made in terms of the false discovery rate. The CMF method only focuses on BLOSUM62dissimilar amino acid pairs as a model of compensatory mutations and integrated them in the calculation of normalized MI-metrics using a doubly stochastic matrix to transform the empirical pair distribution of the column pair. However, the CMF disregards amino acid pair similarity which can be also crucial for the detection of functionally or structurally important sites in MSAs. 
In this study, we present a new method called Quantum Coupled Mutation Finder (QCMF) which extends the CMF algorithm [5] by additionally incorporating amino acid pair similarity. To this end, the QCMF invokes principles from quantum information theory, in particular for the first time in the context of MSA analysis quantum entanglement as a major resource of quantum information. Amino acid pair distributions are replaced by entangled density matrices from quantum mechanics which encompass in our case both empirical pair distributions, possibly transformed by the doubly stochastic matrix used in [5], and pair similarity. Following Capra and Singh [22] who pointed out that it is hard to improve upon metrics based on Jensen-Shannon divergences, we quantify the effect of both amino acid pair similarity and amino acid pair dissimilarity by the quantum Jensen-Shannon divergence between an entangled density matrix and the one that simply represents the amino acid pair frequencies.

The QCMF algorithm is strongly based on the matrix operations that are computationally intensive. When analyzing a single MSA, the computational time of these matrix operations rise very quickly due to the huge number of column pairs. In order to speed up the running time of the QCMF, we implemented its algorithm using Compute Unified Device Architecture (CUDA). CUDA is an efficient parallel computing architecture developed by NVIDIA that utilizes graphic processing units (GPUs) for general-purpose scientific and engineering applications [25]. Nowadays, GPUs are often used for computationally challenging problems in bioinformatics [26-29] and several other scientific fields [30-32].

\section{Results}

Our main focus in this study was to investigate whether quantum information theory based measures could contribute beyond conventional measures to the identification of important residue sites. The Results section of this work twofold. First, to test the functionality of QCMF-significant individual residue sites we analysed the essential sites of two human proteins: epidermal growth factor receptor (EGFR) (pdb entry 2J6M) and glucokinase (GCK) (pdb entry 1V4S). The functionally and structurally important sites of both proteins have been experimentally investigated in several studies previously [33-44] and their positions were summarized in [5] as essential sites. The essential sites of these proteins consist of several non-conserved residue sites which are directly located at or near disease associated amino acid mutation (nonsynonymous single nucleotide polymorphisms (nsSNPs)) sites, catalytic sites, protein binding sites and so on, each of which are likely to affect protein stability or functionality (see [5] and references therein). In addition, residue sites are defined to be in contact according to the "nearby" definition of Nussinov et al. [45] if their carbon major atoms have a distance of less than or equal to $6 \AA$. Consequently, we defined an individual QCMF-significant residue site as "functionally or structurally important" if it corresponds to one of these essential sites.

Second, to further investigate the performance of QCMF and to make a comparison with the previous methods (CMF [5], MIp [6], and PSICOV [18]), we selected a non-redundant set of proteins prepared by Janda et al. [46]. Although the dataset contains 216 proteins, we eliminated a few proteins due to inconsistency between corresponding MSAs and PDB files, so that we finally ended up with a dataset of 153 proteins (see Additional file 1).

The MSAs for each protein, which contain after filtering at least 125 independent sequences, were derived from the HSSP-database [47] that merges primary structure information and tertiary structure information of proteins.

Finally, we define QCMF-significant sites as follows. Let $M$ be an MSA, with the protein of interest being the first row of $M$. A site pair as well as an individual site of the protein are said to be QCMF-significant with respect to the MSA $M$, if they are $\left(\mathbb{Q}_{\text {ent }}, M\right)$-significant or $\left(\mathbb{Q}_{\text {sep }}, M\right)$ significant. The latter two notions and the underlying two co-evolutionary column pair metrics $\mathbb{Q}_{\text {ent }}$ and $\mathbb{Q}_{\text {sep }}$ are defined in the Methods section. If the MSA $M$ is fixed, we speak of $\mathbb{Q}_{\text {ent-significance and }} \mathbb{Q}_{\text {sep-significance, rather }}$ than of $\left(\mathbb{Q}_{\text {ent }}, M\right)$-significance and $\left(\mathbb{Q}_{\text {sep }}, M\right)$-significance, respectively.

\section{QCMF-significant residue sites in the Human Epidermal Growth Factor Receptor (EGFR) protein}

Using the MSA-specific statistical model with a false discovery rate (FDR) of $1 \%$ for both QCMF-metrics, we first determined altogether 2688 out of 26079 non-conserved column pairs as significant in corresponding MSA of human EGFR protein. 631 of these significant pairs were

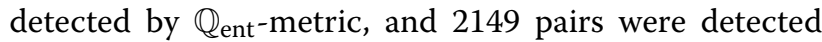

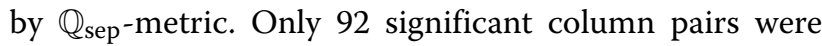
detected by both metrics. After that, utilizing the connectivity degree technique, we predicted in total 33 residue sites in corresponding sequence of human EGFR protein as QCMF-significant (see Additional file 2). 12 of them

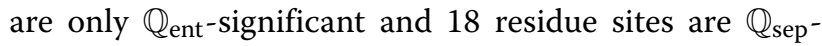
significant, the remaining 3 residue sites (A839, A882 and V902) are both $\mathbb{Q}_{\text {ent }}$-significant and $\mathbb{Q}_{\text {sep-significant. }}$

10 of the QCMF-significant residue sites are in contact with either catalytic residues or critical active site regions for gefitinib binding site in wild type EGFR kinase $[34,37,48]$ (see Figure 1 and Figure 2). Among these sites, the A839 and R841 have been verified as catalytic residue sites through the Catalytic Site Atlas [48]. The T854 is a gefitinib binding site by itself and the residue sites V845 and A859 are also in contact with nsSNP positions K846, 




Figure 1 QCMF-significant residue positions are in contact with catalytic residues in human EGFR protein (PDB-Entry 2J6M). Red spheres denote positions of the catalytic residues. Yellow spheres show the localization of significant adjacent residue positions found by QCMF which are in contact with these catalytic residues. Moreover, the QCMF-significant sites A839 and R841 are also catalytic residues by themselves. Green spheres show the structural localization of nsSNP positions found by QCMF as significant in the EGFR protein. The circles indicate clusters of catalytic residue sites and their significant adjacent sites.

T847 and K860 in human EGFR protein. Moreover, two out of all 33 significant sites are related to disease associated nsSNP positions and their structural localization are illustrated in Figure 1.

Additionally, 13 out of all QCMF-significant sites are referred to as essential sites, each of them are either nearby strictly conserved residues or nsSNPs (see Table 1 ).

According to the essential sites of human EGFR protein, published in [5], we have shown altogether the structural or functional importance of 25 QCMF-significant sites. The remaining 8 significant residue sites (G729, T851, G779, Q820, M825, L927, G930, Y944) do not fall into essential sites and the reason for their significance and their importance in the EGFR protein is currently unclear.

\section{QCMF-significant residue sites in the Human Glucokinase (GCK) protein}

Like human EGFR protein, applying the MSA-specific statistical model with a FDR of $1 \%$ for both QCMF-metrics we identified a total of 9853 out of 69645 non-conserved column pairs as significant in the human GCK protein (pdb entry 1V4S). 6070 of them were $(\mathbb{Q}$ ent,$M$ )-significant and 4232 were detected as $\left(\mathbb{Q}_{\text {sep }}, M\right)$-significant. Only 449 column pairs were detected as significant with respect to both metrics. Thereupon using the connectivity degree technique, we determined altogether 64 residue sites in the human GCK protein as QCMF-significant (see Additional file 3). 30 of them are determined as $\mathbb{Q}_{\text {ent }}{ }^{-}$ significant and further 30 significant residue sites are determined as $\mathbb{Q}_{\text {sep-significant. Only four residue sites }}$ (T82, G223, V253, and G407) are significant based on both metrics.

13 of QCMF-significant sites are in contact with allosteric sites V62, R63, M210, I211, Y214, Y215, M235, V452, V455 and A456 in the human GCK protein. Among these significant sites, the V62, M210, $Y 215$ are allosteric sites by themselves [41] and the T209M, G223S and S453del are related to disease associated nsSNP positions. In addition, there are further five QCMF-significant sites (F123L, G162D, G175R, 


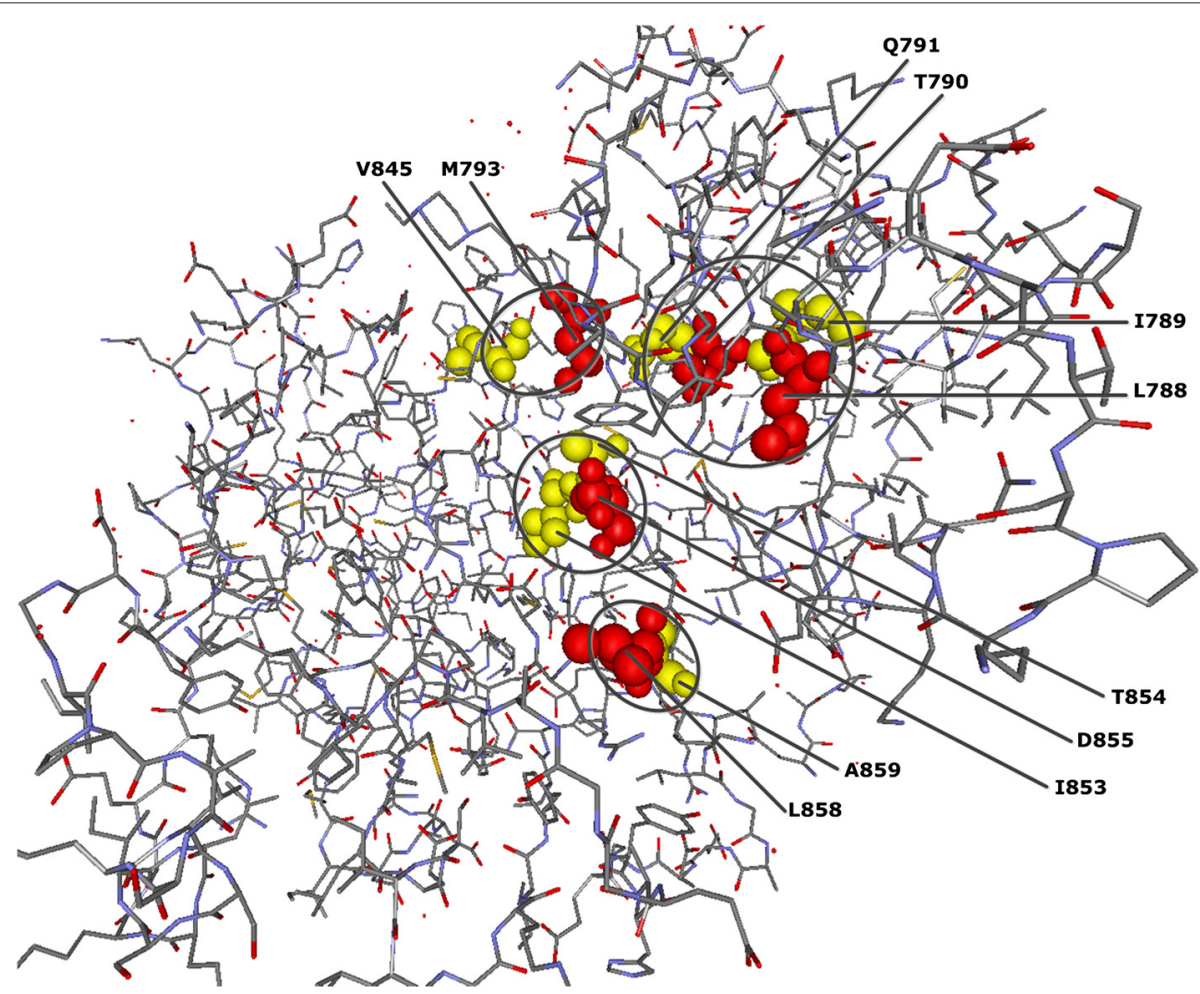

Figure 2 QCMF-significant residue positions are in contact with gefitinib binding sites in human EGFR protein (PDB-Entry 2J6M). Red spheres show the structural localization of the gefitinib binding sites in the wild type kinase. Yellow spheres show QCMF-significant adjacent residue positions which are in contact with these binding sites. Moreover, the QCMF-significant site T854 is also a binding site by itself and interacts with gefitinib binding site D855. The circles indicate clusters of gefitinib binding sites and their significant adjacent sites.

\begin{tabular}{|c|c|c|}
\hline $\begin{array}{l}\text { QCMF-significant } \\
\text { essential sites }\end{array}$ & $\begin{array}{l}\text { Nearby nsSNPs, or strictly } \\
\text { conserved sites }\end{array}$ & Reference \\
\hline$\overline{N 771}$ & $773^{s}$ & [44] \\
\hline G824 & $773^{s}$ & [44] \\
\hline Y827 & $829^{\mathbf{s}}$ & [44] \\
\hline L828 & $829^{\mathbf{s}}$ & [44] \\
\hline V834 & $835^{\mathbf{c}}, 836^{\mathbf{s}}, 860^{\mathbf{s}}$ & {$[44,49]$} \\
\hline Y891 & $892^{\mathbf{s}}, 895^{\mathbf{c}}$ & [44] \\
\hline A822 & $861^{\mathbf{s}}$ & {$[43,49,50]$} \\
\hline V844 & $796^{\mathbf{c}}, 798^{\mathbf{c}}, 852^{\mathbf{c}}$ & - \\
\hline A882 & $884^{\mathbf{c}}, 895^{\mathbf{c}}, 898^{\mathbf{c}}$ & - \\
\hline Y900 & $898^{\mathbf{c}}, 901^{\mathbf{c}}$ & - \\
\hline V902 & $880^{c}, 901^{c}$ & - \\
\hline T909 & $906^{\mathbf{c}}, 936^{\mathbf{c}}$ & - \\
\hline G911 & $906^{c}$ & - \\
\hline
\end{tabular}

$\mathbf{s}:$ non-synonymous snp site, ${ }^{\mathbf{c}}:$ strictly conserved site.
T228M, and E300K,Q) that have been verified as nsSNP positions through annotation databases and previous experimental studies [38-40,42,43,51]. The structural localization of these 18 QCMF-significant sites (contact sites and nsSNPs positions) are illustrated in Figure 3.

Additionally, eight significant sites T149, G170, F171, T206, V207, A208, Q287 and G294 in contact with glucose binding sites (active sites) T168, K169, D204, D205 and E290 in human GCK protein [41] (see Figure 4) where V207 and A208 are also in contact with the allosteric sites M210 and I211.

Moreover, we have also observed that 38 QCMFsignificant sites are further included in essential sites since they are nearby nsSNPs or strictly conserved residues in human GCK protein (see Table 2).

In total, we have demonstrated here that according to the essential sites of GCK, 62 out of 64 QCMF- significant sites are functionally or structurally important for human GCK protein. The remaining two significant residue sites V89 and N283 do not overlap with essential sites and the reason for their significance and their role in the GCK protein is still unclear. 


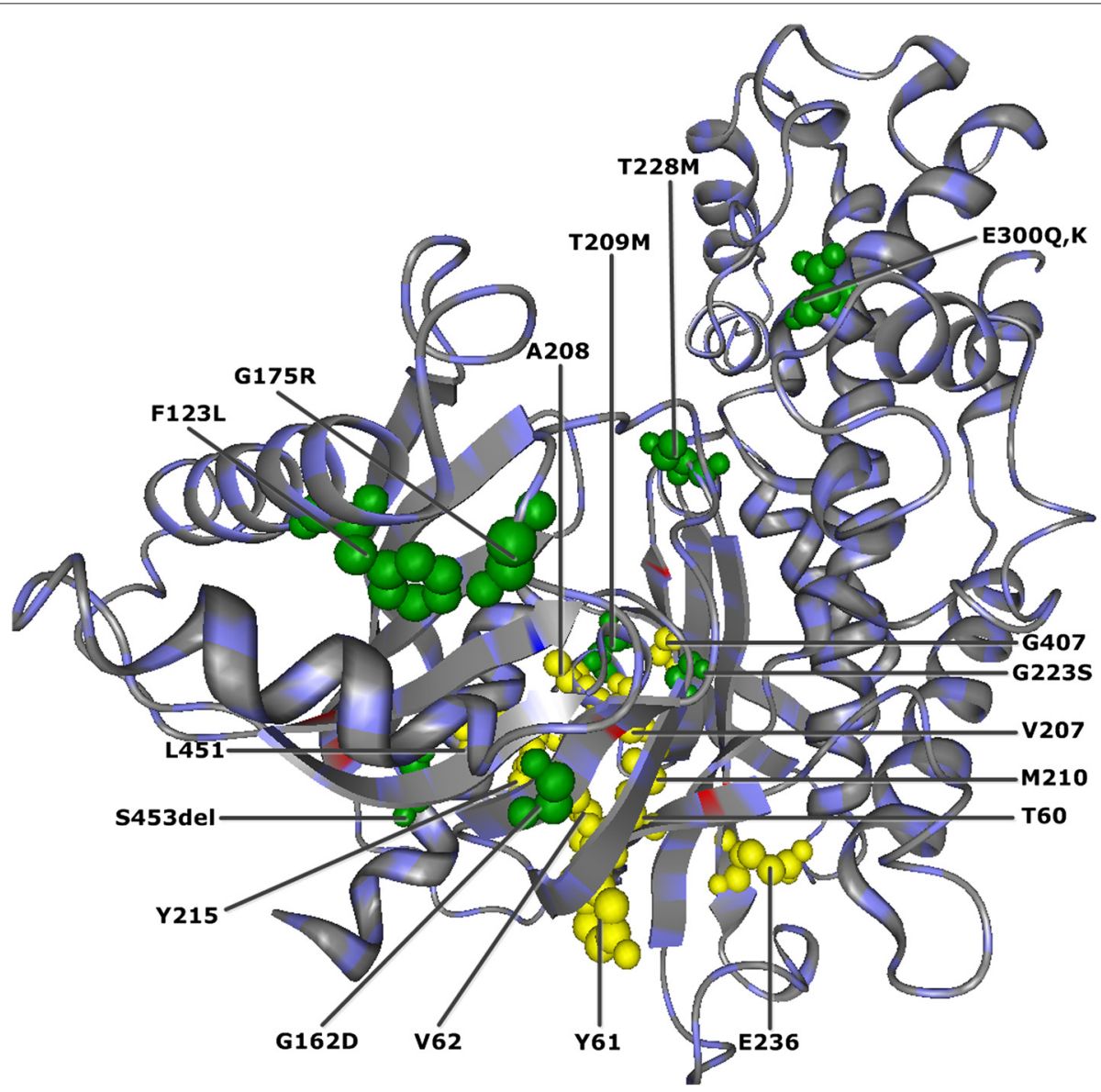

Figure 3 QCMF-significant positions that are either in contact with allosteric sites or related to nsSNPs in human GCK protein (PDB-Entry 1V4S). Yellow spheres correspond to structural localization of ten significant residue sites which are in contact with allosteric sites where V62, M210, and Y215 are denoted as allosteric sites by themselves and they are also in contact with an other allosteric sites. Green spheres indicate eight significant nsSNP positions in the GCK protein. Three of them (T209M, G223S and S453del) are further in contact with allosteric sites M210, I211, V452, V455 and A456.

\section{Individual residue site comparison between} QCMF-significant sites and previous CMF-significant sites We compared QCMF-significant residue sites for both human EGFR and GCK proteins with the significant residue sites given in [5] of the previous CMF-method. The CMF-method detected for both human proteins, 43 sites in EGFR and 72 sites in GCK as significant.

For the EGFR protein we found that the QCMFsignificant residue sites Q791, Q820, G824, K860, Y891, T892, Y900, T909 overlap with results of the CMFmethod. Interestingly, one of the unconfirmed residue sites, the Q820, has been predicted by both QCMFmethod and CMF-method as significant.

For GCK protein, we observed that in total 24 QCMFsignificant sites (T60, T82, N83, F123, F148, T149, F152, H156, F171, N180, T206, T209, T228, E236, G260, L271, S281, N283, Q287, G294, E300, T332, F419 and E443) were also determined by the CMF-method as significant. Although both methods detected residue site N283 as significant, it corresponds to one of the unconfirmed residue sites for GCK, currently.

The CMF has been developed using normalized mutual information (MI) measures in order to detect important residue positions in MSAs. The method mainly focuses on significant BLOSUM62-dissimilar amino acid signals as a model of compensatory mutations and integrates them in the calculation of normalized MI-metrics. As a consequence of mainly taking into account dissimilar amino acid signals, an important part of CMF-significant sites were verified as disease associated nsSNP positions and just a small part of them were located at or near the catalytic sites, allosteric sites and binding sites in both proteins.

Moreover, when statistically evaluating both methods, we have observed that the QCMF significantly outperforms the QCMF-method. The QCMF reaches an improved performance in identifying essential sites from MSAs of both proteins with a significantly higher 




Figure 4 QCMF-significant residue positions are in contact with glucose binding site in human GCK protein (PDB-Entry 1V4S). (A) Red spheres show the structural positions of the glucose binding sites (active sites) and yellow spheres show the localization of significant adjacent residue positions found by QCMF which are in contact with these active sites. The circles indicate clusters of glucose binding sites and their significant adjacent sites.

Matthews correlation coefficient (MCC) value of 0.215 whereas the CMF reaches only a MCC value of 0.133 .

\section{Significant residue pair comparison}

To analyze whether the quantum-information-theorybased measures proposed in this study complements the coventional methods for the detection of correlated (coevolutionary) mutations, we made pairwise comparisons between our new QCMF, MIp [6], PSICOV [18], and CMF [5].

All four methods take as input an MSA satisfying certain admissibility criteria. The problem is that QCMF and CMF output the set of QCMF-significant sites and CMF-significant sites of $M$ 's reference protein, respectively, whereas PSICOV and MIp result in sets of important residue pairs. To make these outputs comparable, we extend them in all cases.

Let $\mathcal{V}_{\mathrm{QCMF}}$ denote the output of QCMF on any admissible MSA $M$. We extend this set to what we call the QCMFsignificant residue network $\mathcal{N}_{\mathrm{QCMF}}:=\left(\mathcal{V}_{\mathrm{QCMF}}, \mathcal{E}_{\mathrm{QCMF}}\right)$ of $M$ as follows. Any two elements of $\mathcal{V}_{\mathrm{QCMF}}$ are connected by an undirected edge belonging to $\mathcal{E}_{\mathrm{QCMF}}$ if and only if the corresponding column pair is QCMFsignificant.

The CMF-significant residue network $\mathcal{N}_{C M F}$ is analogously defined.

In order to get a sufficiently large number MIpsignificant and PSICOV-significant residue pairs, for every input MSA we simply took the top-ranking $10 \%$ as MIp-significant and PSICOV-significant, respectively.

We then utilized the connectivity degree technique in the same way as we did for CMF and QCMF to calculate the set of MIp-significant sites $\mathcal{V}_{\text {MIp }}$ and the set of PSICOV-significant sites $\mathcal{V}_{\text {PSICOV }}$.

For all four methods we used the 90th, the 95th and the 99th percentile as cut-off values.

Finally, the edge sets $\mathcal{E}_{\text {MIp }}$ and $\mathcal{E}_{\text {PSICOV }}$ were determined by full analogy with the calculation of $\mathcal{E}_{\mathrm{QCMF}}$ and $\mathcal{E}_{\mathrm{CMF}}$. Thus we obtained the MIp-significant residue network $\mathcal{N}_{\text {MIp }}$ and the PSICOV-significant residue network $\mathcal{N}_{\text {PSICOV }}$. 
Table 2 QCMF-significant essential sites in the human GCK protein, which are nearby either nsSNPs or strictly conserved sites

\begin{tabular}{|c|c|c|}
\hline $\begin{array}{l}\text { QCMF-significant } \\
\text { essential sites }\end{array}$ & $\begin{array}{l}\text { Nearby nsSNPs or strictly } \\
\text { conserved sites }\end{array}$ & Reference \\
\hline M37 & $36^{\mathbf{s}}, 39^{\mathbf{s}}, 40^{\mathbf{s}}$ & {$[38,39,43,51]$} \\
\hline S76 & $147^{c}$ & \\
\hline$L 79$ & $78^{c}, 80^{\mathbf{c}}, 150^{\mathbf{c}}$ & - \\
\hline T82 & $81^{c}$ & - \\
\hline N83 & $81^{\mathbf{c}}, 108^{\mathbf{s}}, 110^{\mathbf{s}}$ & {$[43,51]$} \\
\hline V86 & $85^{\mathbf{c}}, 106^{\mathbf{s}}$ & {$[38]$} \\
\hline$\$ 127$ & $130^{\mathbf{s}}$ & {$[40]$} \\
\hline$F 148$ & $147^{\mathbf{c}}, 150^{\mathbf{c}, \mathbf{s}}$ & {$[38,39,43,51]$} \\
\hline$F 152$ & $150^{\mathbf{c}, \mathbf{s}}, 151^{\mathbf{c}}$ & {$[39,43,51]$} \\
\hline$P 153$ & $154^{\mathbf{s}}$ & {$[39]$} \\
\hline$H 156$ & $154^{\mathbf{s}}$ & {$[39]$} \\
\hline$A 176$ & $119^{\mathbf{s}}, 175^{\mathbf{s}}$ & {$[43]$} \\
\hline G178 & $164^{c}$ & \\
\hline$N 180$ & $162^{\mathbf{s}}, 182^{\mathbf{s}}$ & {$[38,39,43]$} \\
\hline$L 185$ & $182^{\mathbf{s}}, 188^{\mathbf{s}}$ & {$[39,43,51]$} \\
\hline A201 & $147^{c}, 453^{c}$ & \\
\hline M202 & $147^{c}, 203^{s}$ & {$[43]$} \\
\hline$A 232$ & $223^{\mathbf{s}}, 231^{\mathbf{c}}$ & {$[39,40,51]$} \\
\hline C233 & $223^{\mathbf{s}}, 234^{\mathbf{c}}, 235^{\mathbf{c}}$ & {$[39,40,51]$} \\
\hline V253 & $234^{c}, 254^{c}$ & \\
\hline$F 260$ & $257^{\mathbf{s}}, 258^{\mathbf{c}}, 259^{\mathbf{s}}, 261^{\mathbf{s}}$ & {$[39,43]$} \\
\hline$L 271$ & $274^{c}$ & \\
\hline V277 & $274^{\mathbf{c}}, 278^{\mathbf{c}}, 279^{\mathbf{s}}$ & {$[43]$} \\
\hline S281 & $278^{\mathbf{c}}, 279^{\mathbf{s}}$ & {$[43]$} \\
\hline Y297 & $291^{\mathbf{c}}, 295^{\mathbf{c}}, 299^{\mathbf{c}}, 300^{\mathbf{s}}$ & {$[43]$} \\
\hline M298 & $295^{\mathbf{c}}, 299^{\mathbf{c}}, 300^{\mathbf{s}}$ & {$[43]$} \\
\hline T332 & $295^{c}, 299^{c}$ & \\
\hline V374 & $377^{c}$ & \\
\hline A378 & $377^{\mathbf{c}}, 382^{\mathbf{s}}$ & {$[43]$} \\
\hline A379 & $377^{\mathbf{c}}, 382^{\mathbf{s}}$ & {$[43]$} \\
\hline S383 & $382^{\mathbf{s}}, 385^{\mathbf{s}}$ & {$[43]$} \\
\hline A384 & $382^{\mathbf{s}}, 385^{\mathbf{s}}$ & {$[43]$} \\
\hline A387 & $385^{\mathbf{s}}$ & {$[43]$} \\
\hline S388 & $385^{\mathbf{s}}, 392^{\mathbf{s}}$ & {$[38,43]$} \\
\hline V412 & $226^{\mathbf{s}}, 227^{\mathbf{c}}, 410^{\mathbf{c}}, 414^{\mathbf{s}}, 416^{\mathbf{s}}$ & {$[40,43]$} \\
\hline$F 419$ & $416^{\mathbf{s}}$ & {$[40]$} \\
\hline E443 & $444^{\mathbf{c}}, 445^{\mathbf{c}}, 447^{\mathbf{s}}$ & {$[39]$} \\
\hline G446 & $444^{\mathbf{c}}, 445^{\mathbf{c}}, 447^{\mathbf{s}}, 448^{\mathbf{c}}, 449^{\mathbf{c}}$ & [39] \\
\hline
\end{tabular}

$\mathbf{s}$ : non-synonymous snp site, ${ }^{\mathbf{c}}:$ strictly conserved site.
We performed the method comparison edgeoriented, with the number of overlapping edges as measure. We applied all four methods to the 153 MSAs (see Additional files 1) described at the very beginning of this section and calculated the numbers $\left|\mathcal{E}_{\mathrm{QCMF}}^{(i)}\right|, \quad\left|\mathcal{E}_{\mathrm{CMF}}^{(i)}\right|,\left|\mathcal{E}_{\text {PSICOV }}^{(i)}\right|,\left|\mathcal{E}_{\mathrm{MIp}}^{(i)}\right|,\left|\mathcal{E}_{\mathrm{QCMF}}^{(i)} \cap \mathcal{E}_{\mathrm{MIp}}^{(i)}\right|$, $\left|\mathcal{E}_{\mathrm{QCMF}}^{(i)} \cap \mathcal{E}_{\mathrm{PSICOV}}^{(i)}\right|,\left|\mathcal{E}_{\mathrm{QCMF}}^{(i)} \cap \mathcal{E}_{\mathrm{CMF}}^{(i)}\right|,\left|\mathcal{E}_{\mathrm{MIp}}^{(i)} \cap \mathcal{E}_{\mathrm{PSICOV}}^{(i)}\right|$, $\left|\mathcal{E}_{\text {MIp }}^{(i)} \cap \mathcal{E}_{\mathrm{CMF}}^{(i)}\right|$ and $\left|\mathcal{E}_{\text {PSICOV }}^{(i)} \cap \mathcal{E}_{\mathrm{CMF}}^{(i)}\right|$ on each of them, where the connectivity cut-off ranges over the 90th, the 95th and the 99th percentile, and $i=1,2, \ldots, 153$. Summing up the 153 numbers in each of these groups results in the numbers $\sum_{i=1}^{153}\left|\mathcal{E}_{\mathrm{QCMF}}^{(i)}\right|, \quad \sum_{i=1}^{153}\left|\mathcal{E}_{\mathrm{CMF}}^{(i)}\right|$, $\sum_{i=1}^{153}\left|\mathcal{E}_{\text {PSICOV }}^{(i)}\right|, \quad \sum_{i=1}^{153}\left|\mathcal{E}_{\text {MIp }}^{(i)}\right|, \quad \sum_{i=1}^{153}\left|\mathcal{E}_{\mathrm{QCMF}}^{(i)} \cap \mathcal{E}_{\mathrm{MIp}}^{(i)}\right|$, $\sum_{i=1}^{153}\left|\mathcal{E}_{\mathrm{QCMF}}^{(i)} \cap \mathcal{E}_{\mathrm{PSICOV}}^{(i)}\right|, \quad \sum_{i=1}^{153}\left|\mathcal{E}_{\mathrm{QCMF}}^{(i)} \cap \mathcal{E}_{\mathrm{CMF}}^{(i)}\right|$, $\sum_{i=1}^{153}\left|\mathcal{E}_{\mathrm{MIP}}^{(i)} \cap \mathcal{E}_{\mathrm{PSICOV}}^{(i)}\right|, \quad \sum_{i=1}^{153}\left|\mathcal{E}_{\mathrm{MIp}}^{(i)} \cap \mathcal{E}_{\mathrm{CMF}}^{(i)}\right| \quad$ and $\sum_{i=1}^{153}\left|\mathcal{E}_{\text {PSICOV }}^{(i)} \cap \mathcal{E}_{\mathrm{CMF}}^{(i)}\right|$, which are displayed in Tables 3 and 4 .

Table 3 shows that all methods detect with the same connectivity degree cut-off a comparable number of edges in the corresponding significant residue network.

Table 4 highly suggests that all four methods carry distinct information. The overlap between any two of them is less than or equal to $10 \%$. This indicates that, under the assumption that each of them models important aspects of co-evolution, they complement each other perfectly. In particular, this is true for QCMF as a quantuminformation-science-based service compared with the other three established tools that are based on conventional methods.

Implementation of QCMF: Parallel computing using CUDA The computation of both QCMF metrics (Equations 7 and 8) is strongly based on matrix operations. Therefore, we implement QCMF algorithm using CUDA [25] which is very suitable to perform large number of vector and matrix operations in real time. This results in a dramatic reduction of computational time of QCMF.

In this study, we use the CUDA 4.0 architecture (Toolkit) with several linear algebra libraries such as MAGMA [52], LAPACK [53], BLAS [54], GotoBLAS [55], CUBLAS [25] together (see Figure 5) to speed up the running time of the QCMF algorithm. Since our program requires a cooperative multi threading to not fall in any asynchronicity or locks we extended the magma library with dynamic scheduling features according to [56]. Further, in order to be able to compare the performance, we also implemented the QCMF algorithm onto CPU architecture alone. Both implementations were performed on an Intel Core $^{\mathrm{Tm}} \mathrm{i} 7-3770 \mathrm{~K}$ Processor operating at $3.9 \mathrm{GHz}$, with 16 GB of DDR3 RAM and a GeForce GTX 680 
Table 3 Total number of edges in method-dependent significant residue networks with respect to various connectivity degree cut-offs

\begin{tabular}{l|l|cc}
\hline & & \multicolumn{2}{c}{ Total number of edges in significant residue networks } \\
\cline { 2 - 3 } Connectivy degree cut-off & 90\%th percentile & 95\%th percentile \\
\hline$\sum_{i=1}^{153} \mid \mathcal{E}_{\mathrm{QCMF}}^{(i)}$ & 82561 & 20411 \\
$\sum_{i=1}^{153} \mid \mathcal{E}_{\mathrm{Mlp}}^{(i)}$ & 90636 & $\mathbf{9 9 \%}$ percentile \\
$\sum_{i=1}^{153} \mid \mathcal{E}_{\mathrm{PSICOV}}^{(i)}$ & 80489 & 24094 \\
$\sum_{i=1}^{153}$ & $\mathcal{E}_{\mathrm{CMF}}^{(i)}$ & 87208 & 21596 \\
\hline
\end{tabular}

graphics card using the Ubuntu 13.04 operating system (64-bit version).

Applying the QCMF algorithm for human EGFR protein with CPU alone and with CUDA acceleration, the average computational time of a column pair was 0.7117 seconds and 0.0301 seconds, respectively. Similarly, for human GCK protein, the average computational time of a column pair was 0.6977 seconds with CPU alone and 0.0299 seconds with CUDA acceleration. Consequently, the algorithm took $\sim 310$ minutes for human EGFR protein and $\sim 811$ minutes for GCK protein with CPU alone. On the other hand, applying the CUDA acceleration it took only $\sim 13$ minutes for EGFR and $\sim 39$ minutes for GCK protein. The comparison between the average times indicates that the required computational time of QCMFalgorithm with the CUDA acceleration was significantly faster than with CPU alone (approximately more than 23 times faster).

\section{Methods}

We predict important sites of a protein by detecting co-evolving residues. Our measures of co-evolution are quantum-Jensen-Shannon-divergence-based metrics of column pairs of a multiple sequence alignment, with the protein under study being the reference row. The quantum Jensen-Shannon divergence in turn has the von Neumann entropy as main building block.

The von Neumann entropy was originally defined in the framework of quantum mechanics. We elucidate it in the subsequent section as far as it is necessary to understand our methods. Researchers interested in learning more are referred to the excellent textbook due to Vedral [57]. A comprehensive reference book was published by Nielsen and Chuang [58].

This section is organized as follows. In the first four subsections we recapitulate techniques developed in [5] which we leverage in this study. This concerns the definition of significant site pairs and of significant individual sites, the preparation of the training data set used, and the computation of a doubly stochastic matrix $D$ as our model of compensatory mutations on grounds of two counting matrices $C_{\text {alt }}$ and $C_{\text {null }}$. These two matrices also form the basis of the two amino acid pair similarity matrices $\mathcal{A}_{\text {ent }}$ and $\mathcal{A}_{\text {sep }}$, which in turn give rise to our new quantuminformation-science-based metrics $\mathbb{Q}_{\text {ent }}$ and $\mathbb{Q}_{\text {sep}}$. The last four subsection are dedicated to their definitions.

\section{Significant column pairs and significant position with respect to a certain metric}

Let $M$ be an MSA, where the protein of interest is represented by $M$ 's first row, and let $\mathbb{E}$ be a metric which assigns to every MSA column pair $\left(\gamma_{1}, \gamma_{2}\right)$ a real number $\mathbb{E}\left(\gamma_{1}, \gamma_{2}\right) \in[0,1]$. We call $\mathbb{E}$ a co-evolutionary column pair metric if it models a biologically meaningful co-evolutionary signal: The larger the metric value on $\left(\gamma_{1}, \gamma_{2}\right)$, the more likely co-evolution between position $\gamma_{1}$ and position $\gamma_{2}$ has occurred.

Let $\widehat{p}_{(i, j)}$ be the empirical relative amino acid pair frequency of the $i$-th and the $j$-th amino acid in column pair $\left(\gamma_{1}, \gamma_{2}\right)$, where $i, j=1,2, \ldots, 20$. (When choosing a row of

Table 4 Total number of edges in two networks of different type with respect to various connectivity degree cut-offs

\begin{tabular}{l|ccc}
\hline & \multicolumn{2}{c}{ Total number of common edges in two networks of different type } \\
\cline { 2 - 4 } Connectivy degree cut-off & 90\%th percentile & 95\%th percentile & 99\%th percentile \\
\hline$\sum_{i=1}^{153}\left|\mathcal{E}_{\mathrm{QCMF}}^{(i)} \cap \mathcal{E}_{\mathrm{Mlp}}^{(i)}\right|$ & 898 & 77 & 0 \\
$\sum_{i=1}^{153} \mid \mathcal{E}_{\mathrm{QCMF}}^{(i)} \cap \mathcal{E}_{\mathrm{PSICOV}}^{(i)}$ & 735 & 64 & 0 \\
$\sum_{i=1}^{153}\left|\mathcal{E}_{\mathrm{QCMF}}^{(i)} \cap \mathcal{E}_{\mathrm{CMF}}^{(i)}\right|$ & 4036 & 474 & 1 \\
$\sum_{i=1}^{153}\left|\mathcal{E}_{\mathrm{Mlp}}^{(i)} \cap \mathcal{E}_{\mathrm{PSICOV}}^{(i)}\right|$ & 9094 & 1488 & 11 \\
$\sum_{i=1}^{153}\left|\mathcal{E}_{\mathrm{Mlp}}^{(i)} \cap \mathcal{E}_{\mathrm{CMF}}^{(i)}\right|$ & 3343 & 474 & 6 \\
$\sum_{i=1}^{153}\left|\mathcal{E}_{\mathrm{PSICOV}}^{(i)} \cap \mathcal{E}_{\mathrm{CMF}}^{(i)}\right|$ & 2618 & 368 & 2 \\
\hline
\end{tabular}




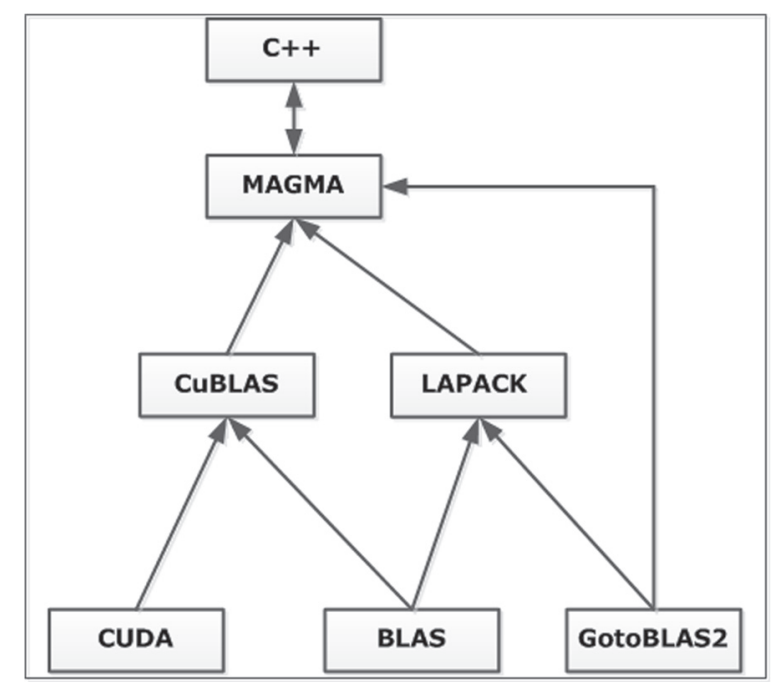

Figure 5 Linking of the CUDA environment using $\mathrm{C}++$.

this column pair by pure chance, acid pair $(i, j)$ is drawn with probability $\widehat{p}_{(i, j)}$.) In the subsequent subsection we recapitulate the way developed in [5] to identify significant columns and significant column pairs with respect to $\mathbb{E}$.

A well-studied example (see $[5,12]$ ) of a co-evolutionary column pair metric is the normalized mutual information

$$
\mathbb{U}\left(\gamma_{1}, \gamma_{2}\right):=2 \cdot \frac{\mathbb{H}\left(\gamma_{1}\right)+\mathbb{H}\left(\gamma_{2}\right)-\mathbb{H}\left(\gamma_{1}, \gamma_{2}\right)}{\mathbb{H}\left(\gamma_{2}+\mathbb{H} \gamma_{2}\right)}
$$

where $\mathbb{H}\left(\gamma_{1}, \gamma_{2}\right), \mathbb{H}\left(\gamma_{1}\right)$, and $\mathbb{H}\left(\gamma_{2}\right)$ denote the Shannon entropy of the empirical pair distribution $\left(\widehat{p}_{(i, j)}\right)_{i, j=1,2, \ldots, 20}$ of the column pair $\left(\gamma_{1}, \gamma_{2}\right)$ and its two marginals.

In order to identify significant column pairs of the MSA under study with respect to the metric $\mathbb{E}$, in [5] we have pointed out, that the distribution of $\mathbb{E}$ can be regarded as a mixture of a background $\beta$-distribution $F_{0}$, an unrelated pair distribution $G_{1}$, and a distribution $G_{2}$ of presumably co-evolving pairs.

The $p$-values $1-F_{0}(\mathbb{E})$ are then uniformly distributed over $[0,1]$ given the underlying $\mathbb{E}$-values are $F_{0}$-distributed. In contrast, $p$-values tend to zero or one, if $\mathbb{E}$-values are $G_{2}$-distributed or $G_{1}$-distributed, respectively.

If, moreover, there is a sub-interval of $[0,1]$ which contains only data from the background distribution, on grounds of a result due to Storey and Tibshirani $[59,60]$ we determined in [5] an MSA-dependent threshold for $\mathbb{E}$ values. A column pair is said to be $(\mathbb{E}, M)$-significant, if its $\mathbb{E}$-value is above the threshold, where the false discovery rate is bounded by a predefined constant.
Figure 6 is a typical pictorial representation of metric distributions which can be treated that way to detect significant pairs.

We applied that model in this study.

We utilized the connectivity degree technique, introduced in [12] and developed further in [5], in order to define the $(\mathbb{E}, M)$-significance of individual residue sites. The connectivity degree of a position $\gamma_{1}$ is the number of positions $\gamma_{2}$ so that the site pair $\left(\gamma_{1}, \gamma_{2}\right)$ is $(\mathbb{E}, M)$-significant. A site of the protein of interest is then called $(\mathbb{E}, M)$-significant, if its connectivity degree cut-off exceeds the 90 -th percentile.

\section{Training data set and pre-processing}

Following [5], a redundancy free set of more than 35000 protein structures is our starting point. This collection was compiled in Rainer Merkl's Lab at the University of Regensburg. The protein structures were taken from the protein data base (http://www.pdb.org/). The PISCES services [61] was applied to assess proteins on sequence similarity and equality of 3D-data. The related MSAs were gathered from the HSSP data base (http://swift.cmbi.ru. $\mathrm{nl} / \mathrm{gv} / \mathrm{hssp} /$ ).

Taking pattern from [12], we filtered every MSA obtained as follows. First, highly similar and dissimilar sequences were deleted to ensure that the sequence identity between any two sequences is at least $20 \%$ and no more than $90 \%$. Second, we removed strictly conserved residue columns, where the percentage of identical residues is greater than 95\%. Third, we eliminated the residue columns which contain more than $25 \%$ gaps. Finally, we discarded all MSAs with less than 125 sequences. More than 17000 MSAs survived the last filtering step. We used approximately 1700 MSAs published in [5] as our training data set which we randomly chose from this set.

\section{Setting up the counting matrices $C_{\text {alt }}$ and $C_{\text {null }}$}

The entries of the two matrices are frequencies of pair substitutions calculated from our training data set described in the foregoing subsection. Informally spoken, matrix $C_{\text {alt }}$ models the signal, whereas $C_{\text {null }}$ reflects the background.

In line with [5], we calculated a signal and a null set of column pairs. The signal set consists of all $(\mathcal{U}, M)-$ significant column pairs, where $M$ ranges over all training MSA. The null set consists of sufficiently many column pairs randomly chosen from every training MSA. For both the signal set and the null set we computed a symmetric $400 \times 400$ integer-valued matrix of frequencies of pair substitutions $C_{\text {alt }}$ and $C_{\text {null }}$. To this end, the method used to compute BLOSUM62 matrices [62] is applied to count residue pair substitutions in MSA column pairs rather than residue substitution in columns. 


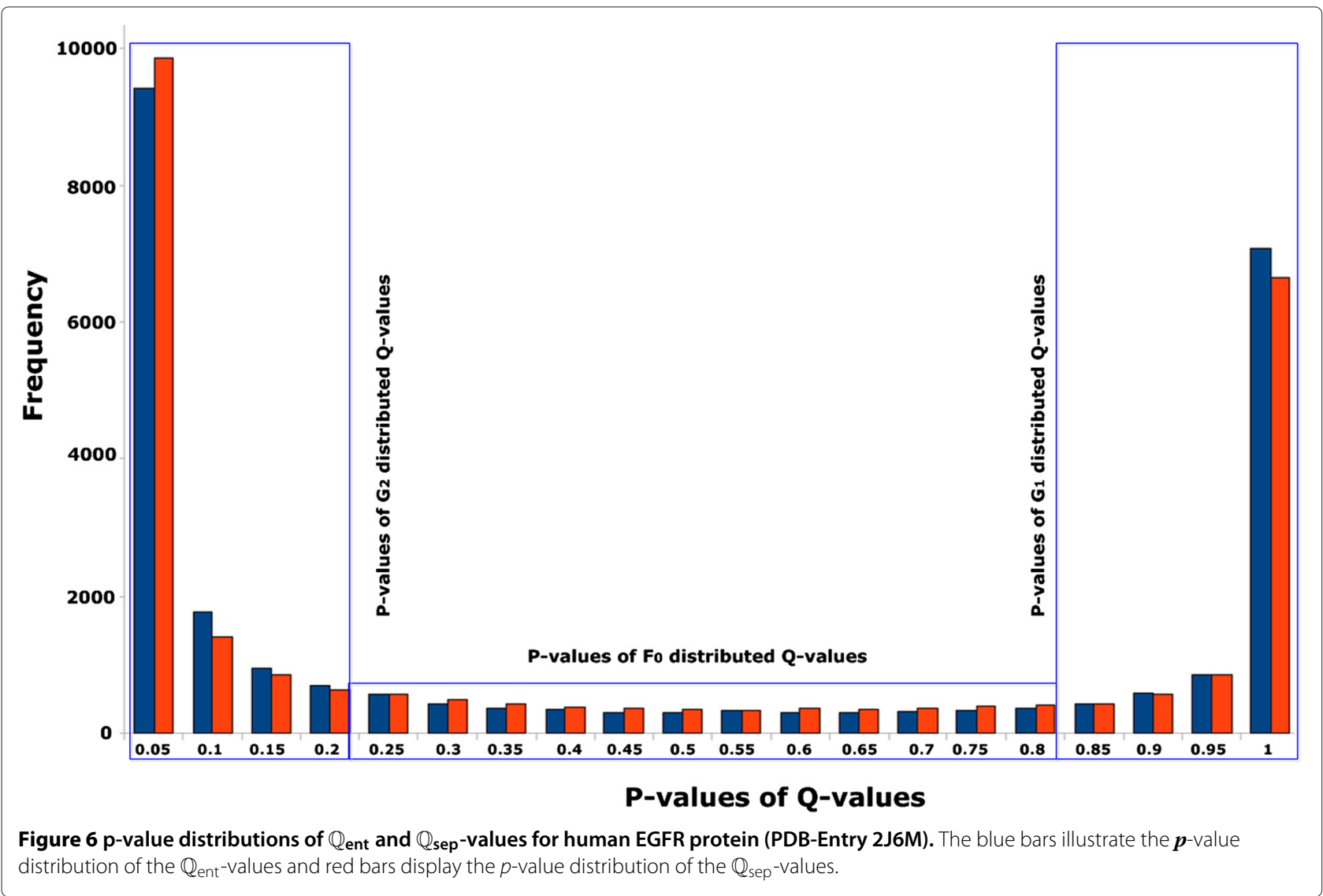

\section{Computing a doubly stochastic matrix $D$}

According to [5], a pair $\left(\left(a_{i}, a_{j}\right),\left(a_{k}, a_{l}\right)\right)$ of amino acid pairs is said to be a formal dissimilar compensatory mutation, if the BLOSUM62 score both of $\left(a_{i}, a_{k}\right)$ and $\left(a_{j}, a_{l}\right)$ is negative.

Using $C_{\text {alt }}$ and $C_{\text {null }}$, we define the matrix $C_{\text {CompMut }}$ by

$$
\begin{aligned}
& C_{\text {CompMut }}\left(\left(a_{i}, a_{j}\right),\left(a_{k}, a_{l}\right)\right) \\
& := \begin{cases}C_{\text {alt }}\left(\left(a_{i}, a_{j}\right),\left(a_{k}, a_{l}\right)\right) & \text { if } \varphi_{\text {CompMut }}\left(\left(a_{i}, a_{j}\right),\left(a_{k}, a_{l}\right)\right)=1 ; \\
0 & \text { otherwise; }\end{cases}
\end{aligned}
$$

where $\varphi_{\text {CompMut }}\left(\left(a_{i}, a_{j}\right),\left(a_{k}, a_{l}\right)\right)=1$ if and only if either $\left(a_{i}, a_{j}\right)=\left(a_{k}, a_{l}\right)$ or $\left(\left(a_{i}, a_{j}\right),\left(a_{k}, a_{l}\right)\right)$ is a formal dissimilar compensatory mutation and

$$
\begin{gathered}
\frac{C_{\text {alt }}\left(\left(a_{i}, a_{j}\right),\left(a_{k}, a_{l}\right)\right)}{\sum_{i^{\prime}, j^{\prime}, k^{\prime}, l^{\prime}} C_{\text {alt }}\left(\left(a_{i^{\prime}}, a_{j^{\prime}}\right),\left(a_{k^{\prime}}, a_{l^{\prime}}\right)\right)} \\
>\frac{C_{\text {null }}\left(\left(a_{i}, a_{j}\right),\left(a_{k}, a_{l}\right)\right)}{\sum_{i^{\prime}, j^{\prime}, k^{\prime}, l^{\prime}} C_{\text {null }}\left(\left(a_{i^{\prime}}, a_{j^{\prime}}\right),\left(a_{k^{\prime}}, a_{l^{\prime}}\right)\right)} .
\end{gathered}
$$

By normalizing $C_{\text {CompMut }}$, we obtain a symmetric matrix $P_{\text {CompMut }}$. For $a_{i}, a_{j}, a_{k}, a_{l}$ ranging over all amino acids, $P_{\text {CompMut }}\left(\left(a_{i}, a_{j}\right),\left(a_{k}, a_{l}\right)\right)$ represents an empirical probability distribution on pairs of amino acid pairs.
We then calculated the symmetric $400 \times 400$-matrix

$$
S_{\text {CompMut }}:=\left(\log \frac{P_{\text {CompMut }}\left(\left(a_{i}, a_{j}\right),\left(a_{k}, a_{l}\right)\right)}{P_{\text {CompMut }}^{\mathrm{b}}\left(a_{i}, a_{j}\right) P_{\text {CompMut }}^{\mathrm{b}}\left(a_{k}, a_{l}\right)}\right)_{\left(a_{i}, a_{j}\right),\left(a_{k}, a_{l}\right)},
$$

where $P_{\text {CompMut }}^{\mathrm{b}}\left(a_{i}, a_{j}\right)$ is the marginal distribution of $P_{\text {CompMut. }}$

Having set all negative entries of $S_{\text {CompMut }}$ to zero, the doubly stochastic matrix $D$ is computed by means of the canonical iterated row-column normalization procedure [63].

The doubly stochastic $D$ is used to linearly transform empirical amino acid pair distributions of column pairs. If the pair distribution is regarded as a 400-dimensional row vector, matrix $D$ is multiplied from the right. If then, for example, the resulting distribution is plugged into Equation 1, column pairs containing formal dissimilar compensatory mutations the $D$-transition probability of which is relatively large tend to be up-scaled.

The idea of the subsequent subsections is to design a model of MSA column pairs that takes formal dissimilar compensatory mutations regarded as pair dissimilarities as well as pair similarities into account. The challenge is to implement this in a way such that these two effects 
interfere but do not interact. This is necessary since a similarity relation is transitive, whereas a dissimilarity relation is not.

\section{Setting up the two counting matrices $C_{\text {ent }}$ and $C_{\text {sep }}$}

We set up two significant pair substitution matrices $C_{\text {ent }}$ and $C_{\text {sep }}$ from $C_{\text {alt }}$ and $C_{\text {null }}$ which form the basis of our new metrics $\mathbb{Q}_{\text {ent }}$ and $\mathbb{Q}_{\text {sep. }}$. The intuition behind $C_{\text {ent }}$ is that the component-wise BLOSUM62-based pair similarity is rescaled, whereas $C_{\text {sep }}$ leads to a new amino acid pair similarity.

$$
\begin{aligned}
& C_{\text {ent }}\left(\left(a_{i}, a_{j}\right),\left(a_{k}, a_{l}\right)\right) \\
& := \begin{cases}C_{\mathrm{alt}}\left(\left(a_{i}, a_{j}\right),\left(a_{k}, a_{l}\right)\right) & \text { if } \varphi_{\mathrm{ent}}\left(\left(a_{i}, a_{j}\right),\left(a_{k}, a_{l}\right)\right)=1 ; \\
0 & \text { otherwise; }\end{cases}
\end{aligned}
$$

where $\varphi_{\text {ent }}\left(\left(a_{i}, a_{j}\right),\left(a_{k}, a_{l}\right)\right)=1$ if and only if either $\left(a_{i}, a_{j}\right)=\left(a_{k}, a_{l}\right)$ or the following two conditions are satisfied. First, the amino acids $a_{i}$ and $a_{k}$ as well as the amino acids $a_{j}$ and $a_{l}$ are BLOSUM62-similar. Second,

$$
\begin{gathered}
\frac{C_{\text {alt }}\left(\left(a_{i}, a_{j}\right),\left(a_{k}, a_{l}\right)\right)}{\sum_{i^{\prime}, j^{\prime}, k^{\prime}, l^{\prime}} C_{\text {alt }}\left(\left(a_{i^{\prime}}, a_{j^{\prime}}\right),\left(a_{k^{\prime}}, a_{l^{\prime}}\right)\right)} \\
>\frac{C_{\text {null }}\left(\left(a_{i}, a_{j}\right),\left(a_{k}, a_{l}\right)\right)}{\sum_{i^{\prime}, j^{\prime}, k^{\prime}, l^{\prime}} C_{\text {null }}\left(\left(a_{i^{\prime}}, a_{j^{\prime}}\right),\left(a_{k^{\prime}}, a_{l^{\prime}}\right)\right)} .
\end{gathered}
$$

$$
\begin{aligned}
& C_{\text {sep }}\left(\left(a_{i}, a_{j}\right),\left(a_{k}, a_{l}\right)\right) \\
& := \begin{cases}C_{\text {alt }}\left(\left(a_{i}, a_{j}\right),\left(a_{k}, a_{l}\right)\right) & \text { if } \varphi_{\text {sep }}\left(\left(a_{i}, a_{j}\right),\left(a_{k}, a_{l}\right)\right)=1 ; \\
0 & \text { otherwise; }\end{cases}
\end{aligned}
$$

where $\varphi_{\text {sep }}\left(\left(a_{i}, a_{j}\right),\left(a_{k}, a_{l}\right)\right)=1$ if and only if either $\left(a_{i}, a_{j}\right)=\left(a_{k}, a_{l}\right)$ or Equation 2 is satisfied.

\section{Calculating the two amino acid pair similarity matrices $\mathcal{A}_{\text {ent }}$ and $\mathcal{A}_{\text {sep }}$}

Recall that a matrix $A$ is positive definite (positive semidefinite), if there is an orthogonal matrix $U$ (defining property $U^{-1}=U^{T}$ ) such that $U A U^{T}$ is a diagonal matrix, where the coefficients in the main diagonal are strictly positive (non-negative).

Let us call a $400 \times 400$-matrix $\mathcal{A}$ a amino acid pair similarity matrix, if $\mathcal{A}$ is positive definite and the entries in the main diagonal are equal to 1 , whereas the off-diagonal elements $\mathcal{A}_{(g, h),(i, j)}((g, h) \neq(i, j))$ are greater than or equal to 0 , but less than 1 .

The entries of an amino acid pair similarity matrix $\mathcal{A}$ are interpreted as follows. The closer $\mathcal{A}_{(g, h),(i, j)}$ to 1 , the more similar are the amino acid pairs $(g, h)$ and $(i, j)$.

Let $C$ be either $C_{\text {ent }}$ or $C_{\text {sep }}$. We define

$$
B_{(g, h),(i, j)}:=\frac{C_{(g, h),(i, j)}^{\alpha}}{\sqrt{\sum_{l, \kappa=1}^{20} C_{(l, \kappa),(i, j)}^{2 \alpha}}},
$$

where $((g, h),(i, j))$ ranges over all possible 160000 indices of pairs of amino acid pairs including the main diagonal, and $\alpha \in(0,1)$ was set to 0.1 in order to enhance the effect of similarity.

Because of the fact, that matrix $B$ is not in any case positive definite, we finally set

$$
\mathcal{A}:=B^{T} B
$$

which is justified by the transitivity of similarity. That way the amino acid similarity matrices $\mathcal{A}_{\text {ent }}$ and $\mathcal{A}_{\text {sep }}$ are obtained from the counting matrices $C_{\text {ent }}$ and $C_{\text {sep }}$, respectively.

Amino acid pair similarity matrices generalize amino acid similarity matrices used by Johansson et al. [24] for evaluating amino acid conservation.

\section{Modeling MSA column pairs and single columns by means of density matrices}

Let $\left(\gamma_{1}, \gamma_{2}\right)$ be a column pair of a multiple sequence alignment, let $\left(\widehat{p}_{(i, j)}\right)_{i, j=1,2, \ldots, 20}$ be the empirical amino acid pair distribution in these columns, let $\left(\widehat{q}_{(i, j)}\right)_{i, j=1,2, \ldots, 20}$ be the linear transform of $\left(\widehat{p}_{(i, j)}\right)_{i, j=1,2, \ldots, 20}$ by the doubly stochastic matrix $D$, and let $\mathcal{A}$ be an amino acid pair similarity matrix.

Recall, that the trace of a matrix is the sum of its coefficients in the main diagonal.

Taking pattern from quantum mechanics, we model column pair $\left(\gamma_{1}, \gamma_{2}\right)$ by a positive semi-definite $400 \times 400$ matrix the trace of which is equal to 1 , a so-called density matrix. Regarding the two distributions $\left(\widehat{p}_{(i, j)}\right)_{i, j=1,2, \ldots, 20}$ and $\left(\widehat{q}_{(i, j)}\right)_{i, j=1,2, \ldots, 20}$ as $400 \times 400$-diagonal matrices the main diagonal of which are formed by the probabilities $\widehat{p}_{(i, j)}$ and $\widehat{q}_{(i, j)}$, respectively, we integrate the classical model into the quantum-mechanics-based one.

Generalizing the approach for amino acid used in [24] to amino acid pairs, our density matrices are of the shape

$$
\rho(\widehat{r}, \mathcal{A}):=\left(\sqrt{\widehat{r}_{(g, h)}} \mathcal{A}_{(g, h),(i, j)} \sqrt{\widehat{r}_{(i, j)}}\right)_{g, h, i, j=1,2, \ldots, 20},
$$

where $\widehat{r}_{(i, j)}$ is either $\widehat{p}_{(i, j)}$ or $\widehat{q}_{(i, j)}(i, j=1,2, \ldots, 20)$. Using this denotation, the diagonal density matrices considered in the preceding paragraph are equal to some $\rho(\widehat{r}, \mathbb{1})$, where $\mathbb{1}$ is the $400 \times 400$-identity matrix.

In this study, we regard individual MSA columns only as components of column pairs. In the classical case, where MSA-column pair $\left(\gamma_{1}, \gamma_{2}\right)$ is modeled by an MSA-dependent amino acid pair distribution $\widehat{r}$ (either $\left(\widehat{p}_{(i, j)}\right)_{i, j=1,2, \ldots, 20}$ or some derivative), the columns $\gamma_{1}$ and $\gamma_{2}$ are represented by the corresponding marginals $\widehat{r}_{1}$ and $\widehat{r}_{2}$ of $\widehat{r}$. 
In quantum information science, the counter part of the marginals $\widehat{r}_{1}$ and $\widehat{r}_{2}$ of $\widehat{r}$ are the partial traces $\operatorname{tr}_{2}(\rho)$ and $\operatorname{tr}_{1}(\rho)$ of $\rho$. They are $20 \times 20$ density matrices defined by

$$
\left(\operatorname{tr}_{1}(\rho)\right)_{i j}:=\sum_{k=1}^{20} \rho_{k k i j} \quad\left(\operatorname{tr}_{2}(\rho)\right)_{i j}:=\sum_{k=1}^{20} \rho_{i j k k},
$$

where $i, j=1,2 \ldots, 20$. As opposed to the indices of the marginals, matrix $\operatorname{tr}_{1}(\rho)$ models column $\gamma_{2}$, whereas matrix $\operatorname{tr}_{2}(\rho)$ represents column $\gamma_{1}$.

\section{Defining our two new metrics $\mathbb{Q}$ ent and $\mathbb{Q}_{\text {sep }}$}

To begin with, we define the von Neumann entropy $\operatorname{VNE}(\rho)$ of a diagonal density matrix $\rho$ as the Shannon entropy of its main diagonal coefficients regarded as a probability distribution.

The crucial property of a density matrix $\rho$ is that there exists an orthogonal matrix $U$ such that $U \rho U^{T}$ is a diagonal density matrix, where the diagonal elements are uniquely determined up to their order. Thus we are justified to finally define

$$
\operatorname{VNE}(\rho):=\operatorname{VNE}\left(U \rho U^{T}\right),
$$

where $U$ is an orthogonal matrix diagonalizing $\rho$ in a way just mentioned.

In principle, the following holds true. The larger the off-diagonal coefficients of the similarity matrix $\mathcal{A}$, the smaller the von Neumann entropy of the density matrix according to Equation 4 compared with the Shannon entropy of the probability distribution $\widehat{r}_{(i, j)}(i, j=$ $1,2, \ldots, 20)$.

In order to compare two density matrices $\rho$ and $\sigma$ of the same dimension, we make use of the quantum JensenShannon divergence:

$$
\operatorname{QJSD}(\rho \| \sigma):=\operatorname{VNE}((\rho+\sigma) / 2)-(\operatorname{VNE}(\rho)+\operatorname{VNE}(\sigma)) / 2
$$

It can be shown that $0 \leq \mathrm{QJSD}(\rho \| \sigma) \leq 1$, where 0 is attained if and only if the two density matrices $\rho$ and $\sigma$ are equal. As oppose to the case of Equation 1, we have thus avoided a normalization.

We are now in a position to define our new two metrics for a certain column pair of a given MSA. As before, the amino acid pair distribution $\widehat{q}$ is given by $\widehat{p} \cdot D$, where $D$ is the $400 \times 400$ doubly stochastic matrix described above, $\widehat{p}$ is the empirical pair distribution of these two columns, and $\mathbb{1}$ is the $400 \times 400$-identity matrix.

Then our first metric $\mathbb{Q}_{\text {ent }}$ is defined by

$$
\mathbb{Q}_{\text {ent }}:=\operatorname{QJSD}\left(\rho\left(\widehat{q}, \mathcal{A}_{\text {ent }}\right) \| \rho(\widehat{p}, \mathbb{1})\right)
$$

(see Equation 4). This metric measures the difference between a density matrix combining rescaled amino acid pair similarity with dissimilar compensatory mutations and the empirical amino acid pair distribution. The index "ent" indicates that here we make use of quantum entanglement, which in turn is a major resource of quantum information science. (Entangled $400 \times 400$-density matrices are those that cannot be represented as a convex combination of Kronecker products of $20 \times 20$-density matrices. Note, that the Kronecker product of density matrices is the analog of the classical product of probability distributions).

Our second new metric $\mathbb{Q}_{\text {sep }}$ is given by

$$
\mathbb{Q}_{\text {sep }}:=\operatorname{QJSD}\left(\operatorname{tr}_{1}\left(\rho\left(\widehat{p}, \mathcal{A}_{\text {sep }}\right)\right) \| \operatorname{tr}_{2}\left(\rho\left(\widehat{p}, \mathcal{A}_{\text {sep }}\right)\right)\right) \text {. }
$$

The density operator $\rho\left(\widehat{p}, \mathcal{A}_{\text {sep }}\right)$ is entangled. However, before finally calculating the metric, we separate the columns of the pair by applying the two partial trace operators.

Using the example of the human EGFR protein (PDBEntry 2J6M), Figure 6 illustrates that the method we developed in [5] to determine significant column pairs is well-applicable for both $\mathbb{Q}_{\text {ent }}$ and $\mathbb{Q}_{\text {sep. The results pre- }}$ sented in this work prove that $\mathbb{Q}_{\text {ent }}$ as well as $\mathbb{Q}_{\text {sep }}$ are powerful co-evolutionary column pair metrics.

\section{Discussion}

Grosse et al. observed in [64] that the Jensen-Shannon divergence (JSD) can be interpreted as mutual information between two (or more) random sources in a special setting particularly appropriate to discriminate between these sources. This is what we need when it comes to predicting important protein sites in an MSA-based approach. It might explain the findings of Capra and Singh [22] on the predictive power of JSD. These two articles encouraged us to utilize quantum Jensen-Shannon divergence (QJSD) in this study. As a side effect, a normalization is not necessary, since quantum Jensen-Shannon divergence, like its classical counterpart, ranges over the real interval $[0,1]$.

Several studies have confirmed the fact that detecting coupled MSA-columns is extremely useful in the prediction of important protein sites (see e.g. [4-6,10-13,65-70]). When using information-theoretic metrics, there is no doubt that it is reasonable to incorporate amino acid pair dissimilarity as well as amino acid similarity in a consistent way such that similarity decreases entropy, whereas dissimilarity increases it. This kind of consistency is important, since entropy is the fundamental building block for most of those metrics. In particular, the Jensen-Shannon divergence between two probability mass functions (pmfs) $p$ and $q$ equals $\mathbb{H}(1 / 2(p+q))-1 / 2(\mathbb{H}(p)+\mathbb{H}(q))$.

In [5] an amino acid pair dissimilarity model for compensatory mutations is presented. A doubly stochastic matrix transforms the empirical amino acid pair distribution of a column pair. 
Rescaled pair similarity of BLOSUM62-similar pairs is to capture an aspect of coupled MSA column pairs orthogonal to the phenomenon of dissimilar compensatory mutations. It models the amino acid pair transition preferences within those column pairs on the average. As suggested by Caffrey et al. [23] as well as Johansson et al. [24], it is promising to incorporate them within the framework of quantum information theory. Therein, density matrices replace pmfs. The counterpart of the entropy of a pmf is the von Neumann entropy (VNE) of a density matrix (see Equation 5). QJSD corresponds then exactly to JSD (see Equation 6).

The challenge was to complement the model presented in [5] by additionally incorporating amino acid pair similarity in a way that the two effects interfere but do not interact. We model an MSA column pair by means of a $400 \times 400$-density matrix, rather than amino acid pair distributions. This provides us with the opportunity to utilize the notion of entanglement, which in turn is a major resource of quantum information. In our model, partial traces play the role of the marginals in the classical case. Pair similarity is reflected by means of positive definite pair similarity matrices (see Equation 3), where positive definiteness, which is a key property of density matrices, can only be ensured by using transitivity of similarity. Since there is no transitivity of dissimilarity, we kept dissimilarity apart from that similarity matrix. Instead, we carried over the CMF dissimilarity model of [5]. Similarity matrix and transformed amino acid pair distribution are joined together by means of Equation 4 in the final step of our density matrix design. That way we minimize the interaction between the two effects of dissimilarity and similarity.

In order to eliminate the noise and to define an MSAdependent threshold for significant column pairs, we followed the line of [5]. The model presented there seems to be of universal applicability. The same is true for the connectivity degree model introduced in [12] and further developed in [5]. Combining them results in a reliable and robust method to determine significant residues.

The results we present in this study show that the vast majority of QCMF-significant residue sites are closely related to functionality and structural stability of both human EGFR and GCK proteins. 10 significant residue sites in EGFR and 19 significant sites in GCK are established as functionally important since they are directly located at or close to catalytic sites, allosteric sites and binding sites which are crucial for maintaining protein functions and for understanding the underlying molecular mechanism (see Figures 1,2,3,4). Additionally, 2 significant sites in EGFR and 8 significant sites in GCK (three of them are also in contact with allosteric sites in GCK) are related to disease associated nsSNP regions of both proteins. As has been noted in [5], most disease-causing mutations at these positions in corresponding sequences destroy structural features of proteins, thus affecting protein stability and often results in loss of protein function.

Although the importance of almost all QCMFsignificant sites are verified through essential sites of both human proteins, there are still eight and two unconfirmed significant sites in EGFR and GCK proteins, respectively, which do not fall into essential sites. It is interesting to note that some of these unconfirmed sites are also referred as significant by CMF [5]. We therefore believe that most of these unconfirmed sites identified by our present method may have an importance for the function and structural stability of both proteins notwithstanding the absence of previous experimental data. A further comparison reveals that the overlaps between the results of the QCMF method and the CMF method are quite low, indicating that both methods detect considerably different sets of residue sites as functionally and structurally important. The comparison results clearly show that considering similar and dissimilar amino acid signals simultaneously, our present method is more sensible to catalytic, allosteric and binding sites, while only focusing on dissimilar signals the previous method deals successfully with nsSNP positions in proteins.

The final comparison between QCMF and CMF on EGFR and GCK proteins is made by inspecting several connectivity degree cut-offs. We initially set it to the 90th percentile at which CMF reaches its maximal MCC value. Going through all possible $n$-th percentiles for $n=$ $80,81, \ldots, 99$, QCMF reaches its maximal MCC value of 0.231 if $n=88$. What we got can be summarized as follows. On the one hand QCMF shows a better performance than CMF in identifying important residue sites. On the other hand QCMF complements CMF. This is because of the fact that the method of QCMF is more information rich than that of CMF. QCMF simultaneously uses similar and dissimilar amino acid pair signals, whereas CMF's method focuses only on amino acid pair dissimilarity.

To confirm the educated guess that QCMF complements conventional methods both from information theory and statistics, we applied QCMF, CMF [5], MIp [6] and PSICOV [18] to the 153 MSAs described at the beginning of the Results section. In sum, each of these methods detects different residue pairs as important, where the pairwise overlap is bounded from above by $10 \%$. The reason for that is that the four methods model different aspects of amino acid pair co-evolution. Consequently, they carry distinct information.

To further improve the specificity of QCMF it is promising to combine its quantum-information-theory-based framework with the direct pair distribution derived in DCA (see e.g. [15] or [16]). 


\section{Conclusions}

In this work, we report a new method, QCMF, applying principles of quantum information theory. In contrast to the previous method CMF which focused on dissimilar amino acid signals, QCMF simultaneously models similar and dissimilar amino acid pair signals in the detection of functionally or structurally important sites. QCMF includes two metrics based on quantum Jensen-Shannon divergence. While the first metric measures compensatory mutations between pairs of columns, the second metric considers the sequence conservation of columns. Results show that QCMF reaches an improved performance in identifying important sites from MSAs and it predicts a quite different set of residue sites as functionally and structurally important (in comparison to the previous method). Further, results indicate that the residue sites found by QCMF are more sensible to catalytic sites, allosteric sites and binding sites than those found by the previous method. On the top of that, a pairwise comparison with existing methods shows that QCMF is complementary to them when it comes to predicting co-evolving residue site pairs.

\section{Additional files}

Additional file 1: Pdb entries. Pdb entries of 153 test proteins.

Additional file 2: EGFR significant sites. QCMF-significant residue sites of the human epidermal growth factor receptor (EGFR) protein.

Additional file 3: GCK significant sites. QCMF-significant residue sites of the human glucokinase (GCK) protein.

\section{Competing interests}

The authors declare that they have no competing interests.

\section{Authors' contributions}

SW developed the model underlying QCMF. EW adjusted the model together with SW and interpreted the results. MG developed the model together with SW, designed and implemented the tool together with SH, SJJ and GD, and interpreted the results together with EW and CM. SH designed and carried out the CUDA programming. GD and SJJ supported SW in developing the model and $M G$ in designing and implementing the tool. CM interpreted the results together with EW and MG. All authors read and approved the manuscript.

\section{Acknowledgements}

We thank Rainer Merkl from Regensburg for his helpful advice and insights at early stages of this project and for comments on the methods.

Mehmet Gültas acknowledges financial support by the Deutsche

Forschungsgemeinschaft (DFG: WA 766/7-1).

Received: 10 June 2013 Accepted: 26 March 2014 Published: 3 April 2014

\section{References}

1. Gloor GB, Martin LC, Wahl LM, Dunn SD: Mutual information in protein multiple sequence alignments reveals two classes of Coevolving positions. Biochemistry 2005, 44(19):7156-7165. [http://pubs.acs.org/ doi/abs/10.1021/bi050293e]. [PMID: 15882054]

2. Wilson K, Walker J: Principles and Techniques of Biochemistry and Molecular Biology. 7th edition. New York: Cambridge University Press; 2010.

3. Altschuh D, Lesk AM, Bloomer AC, Klug A: Correlation of co-ordinated amino acid substitutions with function in viruses related to tobacco mosaic virus. J Mol Biol 1987, 193(4):693-707.
4. Martin LC, Gloor GB, Dunn SD, Wahl LM: Using information theory to search for co-evolving residues in proteins. Bioinformatics 2005 , 21(22):4116-4124.

5. Gültas M, Haubrock M, Tüysüz N, Waack S: Coupled mutation finder: a new entropy-based method quantifying phylogenetic noise for the detection of compensatory mutations. BMC Bioinformatics 2012, 13:225. [http://www.biomedcentral.com/1471-2105/13/225]

6. Dunn SD, Wahl LM, Gloor GB: Mutual information without the influence of phylogeny or entropy dramatically improves residue contact prediction. Bioinformatics 2008, 24(3):333-340.

7. Chakrabarti S, Panchenko AR: Structural and functional roles of Coevolved sites in proteins. PLOS ONE 2010, 5:e8591. [http://dx.doi.org/ 10.1371\%2Fjournal.pone.0008591].

8. Sandler I, Abu-Qarn M, Aharoni A: Protein co-evolution: how do we combine bioinformatics and experimental approaches? Mol BioSyst 2013, 9:175-181. [http://dx.doi.org/10.1039/C2MB25317H]

9. DePristo MA, Weinreich DM, Hartl DL: Missense meanderings in sequence space: a biophysical view of protein evolution. Nat Rev Genet Nat Publishing Group 2005, 6(9):678-687. [http://dx.doi.org/10. 1038/nrg1672]

10. Atchley WR, Wollenberg KR, Fitch WM, Terhalle W, Dress AW: Correlations among amino acid sites in bHLH protein domains: an information theoretic analysis. Mol Biol Evol 2000, 17:164.

11. Tillier ER, Lui TW: Using multiple interdependency to separate functional from phylogenetic correlations in protein alignments. Bioinformatics 2003, 19(6):750-755. [http://bioinformatics.oxfordjournals. org/content/19/6/750.abstract]

12. Merkl R, Zwick M: H2r: Identification of evolutionary important residues by means of an entropy based analysis of multiple sequence alignments. BMC Bioinformatics 2008, 9:151. [http://www. biomedcentral.com/1471-2105/9/151]

13. Gao H, Dou Y, Yang J, Wang J: New methods to measure residues coevolution in proteins. BMC Bioinformatics 2011, 12:206. [http://www. biomedcentral.com/1471-2105/12/206].

14. de Juan D, Pazos F, Valencia A: Emerging methods in protein co-evolution. Nat Rev Genet 2013, 14:249-261.

15. Morcos F, Pagnani A, Lunt B, Bertolino A, Marks DS, Sander C, Zecchina R, Onuchic JN, Hwa T, Weigt M: Direct-coupling analysis of residue coevolution captures native contacts across many protein families. Proc Nat Acad Sci 2011, 108(49):E1293-E1301. [http://www.pnas.org/ content/108/49/E1293.abstract]

16. Marks DS, Colwell LJ, Sheridan R, Hopf TA, Pagnani A, Zecchina R, Sander $C$ : Protein 3D structure computed from evolutionary sequence variation. PLOS ONE 2011, 6(12):e28766.

17. Cheng RR, Morcos F, Levine H, Onuchic JN: Toward rationally redesigning bacterial two-component signaling systems using coevolutionary information. Proc Nat Acad Sci 2014. [http://www.pnas. org/content/early/2014/01/17/1323734111.abstract]

18. Jones DT, Buchan DWA, Cozzetto D, Pontil M: PSICOV: precise structural contact prediction using sparse inverse covariance estimation on large multiple sequence alignments. Bioinformatics 2012, 28(2):184-190.

19. Kamisetty $H$, Ovchinnikov S, Baker D: Assessing the utility of coevolution-based residue-residue contact predictions in a sequence- and structure-rich era. Proc Nat Acad Sci 2013, 110(39):15674-15679. [http://www.pnas.org/content/110/39/15674. abstract]

20. Williamson R: Information theory analysis of the relationship between primary sequence structure and ligand recognition among a class of facilitated transporters. J Theor Biol 1995, 174:179-188.

21. Mirny J, Shakhnovich E: Universally conserved position in protein folds: reading evolutionary signals about stability, folding, kinetics, and function. J Mol Biol 1999, 291:10930-10935.

22. Capra JA, Singh M: Predicting functionally important residues from sequence conservation. Bioinformatics 2007, 23(15):1875-1882.

23. Caffrey DR, Somaroo S, Hughes JD, Mintseris J: Huang ES: Are protein-protein interfaces more conserved in sequence than the rest of the protein surface? Protein Sci 2004, 13:190-2002.

24. Johansson F, Toh H: Relative von Neumann entropy for evaluating amino acid conservation. J Bioinformatics Comput Biol 2010, 
08(05):809-823. [http://www.worldscientific.com/doi/abs/10.1142/ S021972001000494X]

25. NVIDIA CUDA Zone. [http://www.nvidia.com/object/cuda_home_new. html]

26. Liu Y, Wirawan A, Schmidt B: CUDASW++ 3.0: accelerating Smith-Waterman protein database search by coupling CPU and GPU SIMD instructions. BMC Bioinformatics 2013, 14:117. [http://www. biomedcentral.com/1471-2105/14/117]

27. Manavski S, Valle G: CUDA compatible GPU cards as efficient hardware accelerators for Smith-Waterman sequence alignment. BMC Bioinformatics 2008, 9(Suppl 2):S10

28. Lui Y, Maskell D, Schmidt B: CUDASW++: optimizing Smith-Waterman sequence database searches for CUDA-enabled graphics processing units. BMC Res Notes 2009, 2:73.

29. Wirawan A, Kwoh C, Hieu N, Schmidt B: CBESW: Sequence alignment on the Playstation 3. BMC Bioinformatics 2008, 9:377

30. Ufimtsev I, Martinez T: Graphical processing units for quantum chemistry. Comput Sci Eng 2008, 10(6):26-34.

31. Stone J, Hardy D, Ufimtsev I, Schulten K: GPU-accelerated molecular modeling coming of age. J Mol Graph Model 2010, 29(2):116-125.

32. Michalakes J, Vachharajani M: GPU acceleration of numerical weather prediction. Parallel Process Lett 2008, 18(4):531-548.

33. Dixit A, Yi L, Gowthaman R, Torkamani A, Schork NJ, Verkhivker GM: Sequence and structure signatures of cancer mutation Hotspots in protein Kinases. PLOS ONE 2009, 4(10):e7485. [http://dx.doi.org/10.1371 \%2Fjournal.pone.0007485]

34. Yun CH, Boggon TJ, Li Y, Woo MS, Greulich H, Meyerson M, Eck MJ: Structures of lung cancer-derived EGFR mutants and inhibitor complexes: mechanism of activation and insights into differential inhibitor sensitivity. Cancer Cell 2007, 11(3):217-227. [http://www. sciencedirect.com/science/article/pii/S1535610807000281]

35. Zhang H, Berezov A, Wang Q, Zhang G, Drebin J, Murali R, Greene MI: ErbB receptors: from oncogenes to targeted cancer therapies. J Clin Invest 2007, 117(8):2051-2058. [http://www.jci.org/articles/view/32278]

36. Lynch TJ, Bell DW, Sordella R, Gurubhagavatula S, Okimoto RA, Brannigan BW, Harris PL, Haserlat SM, Supko JG, Haluska FG, Louis DN, Christiani DC, Settleman J, Haber DA: Activating mutations in the epidermal growth factor receptor underlying responsiveness of non-small-cell lung cancer to Gefitinib. N Eng/ J Med 2004, 350(21):2129-2139. [http://www. nejm.org/doi/full/10.1056/NEJMoa040938]

37. Balius TE, Rizzo RC: Quantitative prediction of fold resistance for inhibitors of EGFR. Biochemistry 2009, 48(35):8435-8448. [http://pubs. acs.org/doi/abs/10.1021/bi900729a]. [PMID: 19627157].

38. Tinto N, Zagari A, Capuano M, De Simone A, Capobianco V, Daniele G, Giugliano M, Spadaro R, Franzese A, Sacchetti L: Glucokinase gene mutations: structural and genotype-phenotype analyses in MODY children from South Italy. PLOS ONE 2008, 3(4):e1870. [http://dx.plos. org/10.1371\%2Fjournal.pone.0001870]

39. Capuano M, Garcia-Herrero CM, Tinto N, Carluccio C, Capobianco V, Coto I, Cola A, lafusco D, Franzese A, Zagari A, Navas MA, Sacchetti L: Glucokinase (GCK) mutations and their characterization in MODY2 children of Southern Italy. PLOS ONE 2012, 7(6):e38906. [http://dx.doi. org/10.1371\%2Fjournal.pone.0007485]

40. Garcia-Herrero CM, Rubio-Cabezas O, Azriel S, Gutierrez-Nogues A, Aragones A, Vincent O, Campos-Barros A, Argente J, Navas MA: Functional characterization of MODY2 mutations highlights the importance of the fine-tuning of glucokinase and its role in glucose sensing. PLOS ONE 2012, 7:e30518. [http://dx.doi.org/10.1371\%2Fjournal. pone.0038906]

41. Kamata K, Mitsuya M, Nishimura T, ichi Eiki J, Nagata Y: Structural basis for allosteric regulation of the monomeric allosteric enzyme human glucokinase. Structure 2004, 12(3):429-438. [http://www.sciencedirect. com/science/article/pii/S0969212604000474]

42. Hamosh A, Scott AF, Amberger JS, Bocchini CA, McKusick VA: Online Mendelian Inheritance in Man (OMIM), a knowledgebase of human genes and genetic disorders. Nucleic Acids Res 2005, 33(suppl 1):D514-D517. [http://nar.oxfordjournals.org/content/33/suppl_1/D514. abstract]

43. Reichert J, Sühnel J: The IMB Jena image library of biological macromolecules: 2002 update. Nucleic Acids Res 2002, 30:253-254. [http://nar.oxfordjournals.org/content/30/1/253.abstract]
44. Flicek P, Amode MR, Barrell D, Beal K, Brent S, Chen Y, Clapham P, Coates G, Fairley S, Fitzgerald S, Gordon L, Hendrix M, Hourlier T, Johnson N, Kähäri A, Keefe D, Keenan S, Kinsella R, Kokocinski F, Kulesha E, Larsson P, Longden I, McLaren W, Overduin B, Pritchard B, Riat HS, Rios D, Ritchie GRS, Ruffier M, Schuster M, et al.: Ensembl 2011. Nucleic Acids Res 2011, 39(suppl 1):D800-D806. [http://nar.oxfordjournals.org/content/39/ suppl_1/D800.abstract]

45. Keskin O, Tsai CJ, Wolfson H, Nussinov R: A new, structurally nonredundant, diverse data set of protein-protein interfaces and its implications. Protein Sci 2004, 13(4):1043-1055. [http://dx.doi.org/10. 1110/ps.03484604]

46. Janda JO, Busch M, Kuck F, Porfenenko M, Merkl R: CLIPS-1D: analysis of multiple sequence alignments to deduce for residue-positions a role in catalysis, ligand-binding, or protein structure. BMC Bioinformatics 2012, 13:55. [http://www.biomedcentral.com/1471-2105/13/55]

47. Sander C, Schneider R: Database of homology derived protein structures and the structural meaning of sequence alignment. Proteins 1991, 9(1):56-69.

48. Porter CT, Bartlett GJ, Thornton JM: The Catalytic site Atlas: a resource of catalytic sites and residues identified in enzymes using structural data. Nucleic Acids Res 2004, 32(suppl 1):D129-D133. [http://nar. oxfordjournals.org/content/32/suppl_1/D129.abstract]

49. Sherry ST, Ward MH, Kholodov M, Baker J, Phan L, Smigielski EM, Sirotkin K: dbSNP: the NCBI database of genetic variation. Nucleic Acids Res 2001, 29:308-311. [http://nar.oxfordjournals.org/content/29/1/308.abstract]

50. Dixit A, Yi L, Gowthaman R, Torkamani A, Schork NJ, Verkhivker GM: Sequence and structure signatures of cancer mutation hotspots in protein kinases. PLOS ONE 2009, 4(10):e7485. [http://dx.doi.org/10.1371 \%2Fjournal.pone.0030518].

51. Valentinova L, Beer NL, Stanik J, Tribble ND, van de Bunt M, Huckova M, Barrett A, Klimes I, Gasperikova D, Gloyn AL: Identification and functional Characterisation of novel glucokinase mutations causing maturity-onset diabetes of the young in Slovakia. PLOS ONE 2012, 7(4):e34541. [http://dx.doi.org/10.1371\%2Fjournal.pone.0007485]

52. Bosma W, Cannon J, Playoust C: The Magma algebra system. I. The user language. J Symbolic Comput 1997, 24(3-4):235-265. [http://dx.doi. org/10.1006/jsco.1996.0125]. [Computational algebra and number theory (London, 1993)]

53. Anderson E, Bai Z, Bischof C, Blackford S, Demmel J, Dongarra J, Du Croz J, Greenbaum A, Hammarling S, McKenney A, Sorensen D: LAPACK Users' Guide. 3rd edition. Philadelphia, PA: Society for Industrial and Applied Mathematics; 1999

54. An updated set of basic linear algebra subprograms (BLAS). ACM Trans Math Softw 2002, 28(2):135-151. [http://doi.acm.org/10.1145/ 567806.567807]

55. Goto K, Geijn RAvd: Anatomy of high-performance matrix multiplication. ACM Trans Math Softw 2008, 34(3):12:1-12:25. [http://doi. acm.org/10.1145/1356052.1356053]

56. Lifflander J, Evans GC, Arya A, Kale L: Dynamic Scheduling for Work Agglomeration on Heterogeneous Clusters. In Parallel and Distributed Processing Symposium Workshops PhD Forum (IPDPSW), 2012 IEEE 26th International. 2012:2404-2413. doi:10.1109/IPDPSW.2012.297.

57. Vedral V: Introduction to Quantum Information Science (Oxford Graduate Texts). New York: Oxford University Press Inc.; 2006.

58. Nielsen MA, Chuang IL: Quantum Computation and Quantum Information: Cambridge University Press; 2000.

59. Storey JD, Tibshirani R: Statistical significance for genomewide studies. Proc Acad Sci 2003, 100:9440-9445.

60. Walsh B: Multiple comparisons: Bonferroni corrections and false discovery rates. Lecture Notes EEB 581, Department of Ecology and Evolutionary Biology, University of Arizona, 2004.

61. Wang G, Jr RLD: PISCES: recent improvements to a PDB sequence culling server. Nucleic Acids Res 2005, 33(Web-Server-Issue):94-98.

62. Henikoff S, Henikoff JG: Amino acid substitution matrices from protein blocks. Proc Nat Acad Sci 1992, 89(22):10915-10919. [http://www.pnas. org/content/89/22/10915.abstract]

63. Cappellini V, Sommer HJ, Bruzda W, Zyczkowski K: Random bistochastic matrices. J Phys A: Math Theor 2009, 42:23.

64. Grosse I, Bernaola-Galván P, Carpena P, Román-Roldán R, Oliver J, Stanley $\mathrm{HE}$ : Analysis of symbolic sequences using the Jensen-Shannon 
divergence. Phys Rev E 2002, 65:041905. [http://link.aps.org/doi/10.1103/ PhysRevE.65.041905]

65. Göbel U, Sander C, Schneider R, Valencia A: Correlated mutations and residue contacts in proteins. Proteins-Struct Funct Genet 1994, 18(4):309-317.

66. Neher $\mathrm{E}$ : How frequent are correlated changes in families of protein sequences? Proc Nat Acad Sci 1994, 91:98-102. [http://www.pnas.org/ content/91/1/98.abstract]

67. Pollock DD, Taylor WR: Effectiveness of correlation analysis in identifying protein residues undergoing correlated evolution Protein Eng 1997, 10(6):647-657. [http://peds.oxfordjournals.org/content/ 10/6/647.abstract]

68. Lockless SW, Ranganathan R: Evolutionarily conserved pathways of energetic connectivity in protein families. Science 1999, 286(5438):295-299. [http://www.sciencemag.org/content/286/5438/295. abstract]

69. Dekker JP, Fodor A, Aldrich RW, Yellen G: A perturbation-based method for calculating explicit likelihood of evolutionary co-variance in multiple sequence alignments. Bioinformatics 2004, 20(10):1565-1572.

70. Codoner FM, Fares M: Why should we care about molecular coevolution? Evol Bioinform 2008, 4:29-38.

doi:10.1186/1471-2105-15-96

Cite this article as: Gültas et al: Quantum coupled mutation finder: predicting functionally or structurally important sites in proteins using quantum Jensen-Shannon divergence and CUDA programming. BMC Bioinformatics 2014 15:96.

\section{Submit your next manuscript to BioMed Central and take full advantage of:}

- Convenient online submission

- Thorough peer review

- No space constraints or color figure charges

- Immediate publication on acceptance

- Inclusion in PubMed, CAS, Scopus and Google Scholar

- Research which is freely available for redistribution

Submit your manuscript at www.biomedcentral.com/submit 
9.3 Appendix C: PDB enteries of training protein MSAs

9.3 Appendix C: PDB enteries of training protein MSAs 


\section{PDB Enteries of Training MSAs}

Table 9.1: Pdb enteries of training MSAs.

\begin{tabular}{|c|c|c|c|c|c|c|c|}
\hline $2 V 4 E A$ & 1 1CKTA & $2 E O F A$ & $1 T O 9 A$ & $3 B Q 7 A$ & $3 E W S A$ & $2 V M Q A$ & $1 C O 8 A$ \\
\hline $1 Z Y E A$ & $1 D U N A$ & $2 H W 5 A$ & $2 G B R A$ & $4 G T U A$ & $1 G G O A$ & $1 K Y 9 A$ & $1 Y Y 9 A$ \\
\hline $2 E T 1 A$ & $2 B H J A$ & $1 C L 7 H$ & $1 H V 9 A$ & $1 N M L A$ & $1 P Z 1 A$ & $3 C 05 A$ & $2 N Q M A$ \\
\hline $2 R 0 O A$ & $2 W F Q A$ & $3 C Y F A$ & $1 U N 3 A$ & $2 O T U B$ & $1 I N D L$ & $3 E S Y A$ & $1 B M T A$ \\
\hline $2 Q 3 O A$ & $2 D J Q A$ & $1 M C I A$ & $1 P T Q A$ & $1 Y 65 \mathrm{~A}$ & $2 J 0 I A$ & $1 O V 9 A$ & $2 J J Y A$ \\
\hline $1 Y K K B$ & $2 V X L A$ & $1 C Q G A$ & $1 U D C A$ & $2 A 0 W A$ & $1 F Y T B$ & $1 B L 3 A$ & $1 R 36 A$ \\
\hline $2 P 79 A$ & $2 P T 6 A$ & $1 A Q K L$ & 1 IIRBA & $3 D G Z A$ & $2 I F Y A$ & $1 K E N L$ & $1 I 88 \mathrm{~A}$ \\
\hline $1 R U Z H$ & $3 D 5 T A$ & $1 B U H B$ & $3 B 84 A$ & $1 W H I A$ & $2 I S S A$ & $2 C U B A$ & $2 Q Q N H$ \\
\hline $2 D W B A$ & $2 E A O A$ & $1 S 9 P A$ & $2 E M X A$ & $2 F T S A$ & $2 F E M A$ & $1 W 1 B 1$ & $1 N P K A$ \\
\hline $1 Q K W A$ & $1 X Q C A$ & $2 B 1 U A$ & $3 F 9 P A$ & $1 Z U T A$ & $2 Y R H A$ & $2 G O Y A$ & $2 F F Y A$ \\
\hline $1 T Y B E$ & $1 R 9 N A$ & $3 B T 8 A$ & $2 V 8 Q E$ & $1 S J J A$ & $2 I Y 0 B$ & $2 Z J B A$ & $1 U M N A$ \\
\hline 1PTZA & IIOLA & $2 R 4 R H$ & 2CPUA & $2 E O H A$ & $1 T 5 B A$ & $5 B J 3 A$ & $1 D 6 M A$ \\
\hline $1 V H P A$ & $1 I F V A$ & $1 Q O R A$ & $3 F F 1 A$ & $2 D 5 Y A$ & $1 M X S A$ & $2 Q A P A$ & $2 G I X A$ \\
\hline $5 R E Q A$ & $2 F G Q X$ & $2 I P M A$ & $1 O 4 R A$ & $3 B E A A$ & $1 Y I U A$ & 2RHEA & $1 T 5 X A$ \\
\hline $3 C S W A$ & $2 Z 2 Y A$ & $1 W Y O A$ & $2 E 9 U A$ & $1 P 3 O B$ & $1 C 0 F A$ & $1 C Y W A$ & $2 H Q K A$ \\
\hline $1 P R G A$ & $2 O W G A$ & $1 A W 2 A$ & $1 Z 1 D B$ & $2 V 3 X A$ & $5 N L L A$ & $2 F 3 L A$ & $1 C \tilde{U} K A$ \\
\hline 2ETRA & 2 GIVA & $1 V L Z B$ & $2 V F H A$ & $2 B A 0 E$ & $1 P K U A$ & IOUIA & $1 A W 8 E$ \\
\hline 2HANA & 1SSMA & $1 N E K C$ & 1РPAA & $1 A Y M 4$ & $3 H X P A$ & $2 K 5 S A$ & $3 F 0 U X$ \\
\hline $2 R F J A$ & $1 U G A A$ & $1 O M W G$ & $1 P 4 S A$ & $3 D 01 A$ & $2 A D A A$ & $1 H 25 C$ & $2 V S U C$ \\
\hline $1 T 2 Q L$ & $1 A 5 E A$ & $2 Z 83 A$ & $1 P 4 W A$ & 2ITWA & $1 Q T W A$ & $1 U 8 S A$ & 1DJEA \\
\hline $3 G L 1 A$ & $2 A Y M A$ & $3 D 8 X A$ & $1 F H J A$ & $3 G K E A$ & $2 V P J A$ & $1 R 8 M E$ & $2 B I O A$ \\
\hline $2 J B 9 A$ & $8 C A 2 A$ & $2 \mathrm{CHGA}$ & $2 P 71 A$ & $1 P M T A$ & $1 J 2 E A$ & $1 V P 7 A$ & 3CZMA \\
\hline $1 I A G A$ & $2 O G H A$ & $2 \mathrm{CH} 4 \mathrm{~W}$ & $2 V M K A$ & $1 T A B I$ & $1 B 5 Z A$ & $2 R 69 H$ & $1 C S 8 A$ \\
\hline $1 B 8 I B$ & $1 B 50 A$ & 1JDIA & $2 K 1 Z A$ & $1 G V L A$ & $2 V L 9 A$ & $2 U W 1 B$ & $1 I 92 A$ \\
\hline $1 Y 62 A$ & $1 F 2 Z A$ & $1 H V F A$ & $1 C C U A$ & $2 G Z O A$ & $1 A G 9 A$ & $2 B B L A$ & $1 N F I E$ \\
\hline $1 M E X L$ & $1 G F G A$ & $1 T Z P A$ & $3 B W 3 A$ & $1 B B I A$ & $1 Z S 6 A$ & $1 A 5 Z A$ & $2 Q E J C$ \\
\hline $1 U H E B$ & $1 B A F H$ & $1 Q Q J A$ & $3 B 9 J B$ & $2 Z M 1 A$ & $1 L 3 W A$ & $1 G F 6 A$ & $2 Q 4 P A$ \\
\hline $2 V F B A$ & $1 E J I A$ & $3 F L C O$ & $1 W S Z A$ & $2 F 91 A$ & $2 F S V C$ & $1 I Q 3 A$ & $1 M J 8 L$ \\
\hline $1 O J X A$ & $2 J F P A$ & 1OVAA & $1 F A I L$ & $1 A 2 X A$ & 1PKRA & $3 C L U D$ & $3 E D Y A$ \\
\hline $1 M 93 C$ & $1 Q K F A$ & $2 D N M A$ & $1 A 6 T A$ & $1 Y M G A$ & $1 T 3 I A$ & $1 Z L 9 A$ & $3 B Q Y A$ \\
\hline $1 H B R B$ & $1 K 8 X B$ & $2 E D 3 A$ & $1 B 8 G A$ & $1 Y A G A$ & $1 G 9 X A$ & $2 R G 3 A$ & $1 Y A C A$ \\
\hline $2 P 77 A$ & $2 R I V B$ & $2 P B I B$ & $1 H V U A$ & $1 Q F 5 A$ & $2 V 36 A$ & $1 F 4 D A$ & $2 O D B B$ \\
\hline 2PAEA & $2 O T G C$ & $2 I P C A$ & $1 Q 3 I A$ & $1 H W 7 A$ & $1 B P V A$ & $3 G 3 D A$ & $1 Q O G A$ \\
\hline $2 A 9 H A$ & $1 Y 7 T A$ & $1 L B B A$ & $1 N G 9 A$ & $3 D 5 F A$ & $3 C W B A$ & $2 E W 8 A$ & $1 D T G A$ \\
\hline $1 C L 7 L$ & $1 E 20 A$ & $1 G N 6 A$ & $2 P 6 B B$ & $1 U S M A$ & $2 M A S A$ & $1 E C F A$ & $2 Q L 9 B$ \\
\hline $2 Q Q U A$ & $3 D 21 A$ & $3 H L U A$ & $3 H Y G A$ & $2 Q O B A$ & $1 F 54 A$ & $1 C T A A$ & $2 H 79 A$ \\
\hline
\end{tabular}


9.3 Appendix C: PDB enteries of training protein MSAs

Table 9.1 Pdb enteries of training MSAs - continued from previous page

\begin{tabular}{|c|c|c|c|c|c|c|c|}
\hline $2 W 2 N E$ & $\overline{1 N Q B A}$ & $1 Z H 9 A$ & $2 E 2 R A$ & $1 H W U A$ & $2 H Z I A$ & $1 F K O A$ & $2 B W 4 A$ \\
\hline $1 B I Z A$ & $1 R V F L$ & $2 H Y 0 A$ & $1 M 40 A$ & $1 M F 8 B$ & $1 Z 95 A$ & $2 Z D 2 A$ & $2 I U F A$ \\
\hline $2 G R R A$ & $3 C T Z A$ & $1 O 3 S A$ & $1 F S K B$ & $2 J O 6 A$ & $1 L D L A$ & $3 D O K A$ & $1 M P 5 A$ \\
\hline $2036 A$ & $1 M Y 5 A$ & $1 J 19 A$ & $1 M 7 N A$ & $1 C 5 C L$ & $1 V H 7 A$ & $1 J I 8 A$ & $1 J 8 Y F$ \\
\hline $1 G Q R A$ & $2 J 58 A$ & $1 X H G A$ & $2 H 88 A$ & $1 R T 3 B$ & $1 K Z 9 A$ & $2 H Z L A$ & $2 U Z C A$ \\
\hline $1 A N 2 A$ & $1 C G P A$ & $1 N D 9 A$ & $2 V S 4 A$ & $1 I R D A$ & $2 V 55 B$ & $2 F 7 X E$ & $3 M A T A$ \\
\hline $1 A T Y A$ & $1 X 7 Z A$ & $1 B C Z A$ & $2 W 69 A$ & $2 E R 8 A$ & $1 U 6 Z A$ & $2 W 72 A$ & $2 N T 8 A$ \\
\hline $1 O 2 F B$ & $3 H M M A$ & $1 Q L 9 A$ & $1 F S A A$ & $1 T M F 3$ & $2 Q V 1 C$ & $2 W 1 V A$ & $1 A T N A$ \\
\hline $3 C B J A$ & $1 Q J O A$ & $2 P 91 A$ & $1 X F 7 A$ & $1 G Y P A$ & $1 G R O A$ & $1 A D 1 A$ & $2 P 4 V A$ \\
\hline $2 O N 5 A$ & $2 Z P B A$ & $1 Q G K B$ & $1 B W W A$ & $2 A Q M A$ & $2 B M 1 A$ & $2 J N P A$ & $1 W 0 Z U$ \\
\hline $2 Q P U A$ & $1 X 7 A L$ & $3 P B H A$ & $2 E 09 A$ & $1 Y 59 T$ & $1 R H S A$ & $1 I H 0 A$ & $1 Y J R A$ \\
\hline $1 F I 3 A$ & $1 Y F D A$ & $2 B 3 H A$ & $1 W 0 T A$ & $3 F Y S A$ & $1 P K L A$ & $3 F 6 U L$ & $1 Y D B A$ \\
\hline $3 H H H A$ & $3 E P F 3$ & $1 I Q Q A$ & $2 C C G A$ & $1 V 2 X A$ & $1 N O E A$ & $2 V H 9 A$ & $2 K 79 A$ \\
\hline $2 V G L S$ & $3 B V 6 A$ & 1ISNA & $2 Q R Z A$ & $3 B Z X B$ & $2 K B O A$ & $1 A W 8 A$ & $2 G O L A$ \\
\hline $2 Y Q C A$ & $1 Q 6 O A$ & $2 J L J A$ & $2 H L P A$ & $2 B D Q A$ & $3 C 5 Q A$ & $2 G 38 A$ & $1 P 4 C A$ \\
\hline $3 B D M D$ & $2 A P S A$ & $1 M Z B A$ & $2 G J 3 A$ & $3 E 22 A$ & $1 Y I 8 B$ & $1 X Y K A$ & $2 F V C A$ \\
\hline $2 Q Q N L$ & $2 O P O A$ & $3 G K A A$ & $1 E J R C$ & $1 T W Y A$ & $1 A 6 V H$ & $1 W C D J$ & $3 B 6 B A$ \\
\hline $1 U H F A$ & 2EOLA & $1 J P T L$ & $1 Q 5 X A$ & $3 E 7 C A$ & 2QIEA & $2 E S N A$ & $2 E T J A$ \\
\hline $1 Y B Z A$ & $1 O S P O$ & $3 I 8 V A$ & $1 U Z 6 E$ & $1 Z B R A$ & $3 G F T A$ & $2 H 1 V A$ & $1 E 3 I A$ \\
\hline $2 Z 26 A$ & $2 Q 3 Y A$ & $1 P 7 K A$ & $2 N Y B A$ & $2 O 5 K A$ & $3 A I G A$ & $1 I 7 H A$ & $2 U W 2 A$ \\
\hline $1 Z 8 Y J$ & $1 H L 4 A$ & $1 S I 2 A$ & $1 I N 0 A$ & $1 Y X 5 B$ & $2 V V 6 A$ & $1 W 74 A$ & $1 N W 2 A$ \\
\hline $1 N 0 X H$ & $1 T M O A$ & $3 D 3 P A$ & $2 U U M X$ & $1 U G K A$ & $1 O X S C$ & $3 I 4 S A$ & $3 F W Y A$ \\
\hline $1 D D K A$ & $2 G H 9 A$ & $2 Q S Q A$ & $2 J U Z A$ & $1 Z 3 S A$ & $3 H N N A$ & $3 H J B A$ & $1 Z 7 Z 1$ \\
\hline $2 G I 4 A$ & $1 M H W A$ & $2 A X Y A$ & $2 F H T A$ & $2 S N W A$ & $1 E S O A$ & $2 G Q Q A$ & $2 H D C A$ \\
\hline $1 K 9 U A$ & $2 Z 39 A$ & $2 G 6 A A$ & $2 B H 8 A$ & $1 J Z 8 A$ & $1 A T G A$ & $2 R D 1 A$ & $1 T A 8 A$ \\
\hline $3 D D K A$ & $1 W 9 J A$ & $1 S N O A$ & $2 Q V 1 A$ & $1 P 5 J A$ & $1 T Z A A$ & $3 D A J A$ & $1 G W T A$ \\
\hline $1 S A U A$ & $1 W G 2 A$ & $2 G V 0 A$ & $3 C 3 T A$ & $1 J 95 A$ & $2 G M L A$ & $1 L H 0 A$ & $2 D J 6 A$ \\
\hline $2 I U E A$ & $1 K T B A$ & $1 S 1 T A$ & $3 G L B A$ & $1 G Q 2 A$ & $1 R D A A$ & $1 S 3 I A$ & $1 L 8 B A$ \\
\hline $1 T 13 A$ & $1 Y C L A$ & $1 V J A U$ & $2 A S C A$ & $2 G Q R A$ & $2 O M Z A$ & $2 I V 1 A$ & $2 H F G L$ \\
\hline $2 Z U R X$ & $1 Y A B A$ & $3 G 3 N A$ & $3 D L P X$ & $1 I 5 E A$ & $1 S V X B$ & $2 F L 5 A$ & $1 K Z M A$ \\
\hline $1 T X 2 A$ & $2 I O 0 B$ & $3 H H F A$ & $3 B 3 D A$ & $2 V E D A$ & $2 P 2 B A$ & $1 C 1 E H$ & $2 D P 3 A$ \\
\hline $1 N T Y A$ & $2 B Z R A$ & $2 B I G A$ & $3 D E X A$ & $3 E R H A$ & $1 W 8 B L$ & $1 S Y I A$ & $1 C 2 O A$ \\
\hline $3 H P 7 A$ & $1 U F V A$ & $1 D 3 A A$ & $1 S W 1 A$ & $1 M O D A$ & $3 B N D A$ & $2 F 9 O A$ & $1 A 6 F A$ \\
\hline $1 F R B A$ & $1 D E J A$ & $3 D Z Y D$ & $2 R G D A$ & $1 B V K B$ & $1 Z D 9 A$ & $3 B C 7 A$ & $3 D 9 A L$ \\
\hline $1 Z 3 I X$ & $1 E 1 D A$ & $1 G 7 J A$ & $1 E 9 E A$ & $1 D B 3 A$ & $1 S V I A$ & $1 R 64 A$ & $1 Z 07 A$ \\
\hline $1 E Q 2 A$ & $5 C A 2 A$ & $1 J 6 Y A$ & $1 J N R B$ & 2JOTA & $2 O A X A$ & $2 H E D A$ & $2 C U 7 A$ \\
\hline $1 C G J I$ & $1 P C A A$ & $3 G L 6 A$ & $1 U N 2 A$ & $2 E I G A$ & $2 Z H G A$ & $1 Y 791$ & $1 Z G 4 A$ \\
\hline $1 Z 7 B A$ & $1 P E 0 A$ & $1 F P S A$ & $1 N B M D$ & $2 C B Y A$ & $1 S J 1 A$ & $1 B R M A$ & $2 N R L A$ \\
\hline $4 P B G A$ & $1 Y K T B$ & $1 Q 52 A$ & $1 A G 8 A$ & $1 T 11 \mathrm{~A}$ & $1 M 8 V A$ & $2 Q W 7 A$ & $2 N Z D C$ \\
\hline $1 Q A C A$ & $2 P C J A$ & $2 E P 0 A$ & $3 D Q G A$ & 2JEXA & $2 E P V A$ & $3 C X 5 J$ & $2 C 5 L A$ \\
\hline $1 \widetilde{D} G F A$ & $1 C 6 R A$ & $2 Q C U A$ & $2 R G X A$ & $2 I 24 N$ & $1 N E E A$ & $1 F N D A$ & $1 Y 4 R B$ \\
\hline $2 B 8 T A$ & $2 V I I A$ & $2 Z 6 Q A$ & $2 Q M X A$ & $2 I U 8 A$ & $1 F 99 B$ & $1 B 0 T A$ & $2 Z 5 I I$ \\
\hline $1 E A E A$ & $1 K T R L$ & $2 T E C I$ & $3 F S 2 A$ & $2 I D 4 A$ & $3 I Y 7 A$ & $1 H 8 B A$ & $2 P I A A$ \\
\hline $1 M 5 A B$ & $2 B M O A$ & $1 Q D E A$ & $3 F K W A$ & $2 Z A 7 A$ & $1 A 0 J A$ & $1 Y E Z A$ & $1 U E K A$ \\
\hline $1 R V X B$ & $1 Q 7 \mathrm{JA}$ & $1 V S 3 A$ & $1 K 1 C A$ & $1 \mathrm{JO} 8 \mathrm{~A}$ & $2 A U V A$ & $1 J B B A$ & $1 O 9 P A$ \\
\hline $2 Z O K A$ & ЗHOAA & $1 M M 3 A$ & $3 D R S A$ & $2 E N C A$ & $1 K D C A$ & $3 E C 5 A$ & $1 C R K A$ \\
\hline $1 I S 8 A$ & 3HILA & $2 O F 2 A$ & $1 N 1 J A$ & $3 C X 5 C$ & $1 P Y 5 A$ & $2 N W L A$ & $1 X D 8 A$ \\
\hline
\end{tabular}


Table 9.1 Pdb enteries of training MSAs - continued from previous page

\begin{tabular}{|c|c|c|c|c|c|c|c|}
\hline $1 K A W A$ & $1 U L Q A$ & $\overline{1 K E A A}$ & $2 Q O 4 A$ & $3 G K N A$ & $2 Q 66 A$ & $2 I F 2 A$ & $1 Q H H A$ \\
\hline $1 E 1 C A$ & $2 M H A A$ & $1 C M Z A$ & $1 B P T A$ & $2 V D 2 A$ & $1 N C A H$ & $3 I L 8 A$ & $1 T S Z A$ \\
\hline $2 D Q 3 A$ & $2 B Q H A$ & $1 T M 1 I$ & $1 F P O A$ & $2 F 0 I A$ & $1 B I Y A$ & $3 C 1 Y A$ & $2 P T Q A$ \\
\hline $1 H V E A$ & $2 D F B A$ & $1 A 40 A$ & $2 P 6 K A$ & $1 K T R H$ & $1 H 8 S A$ & $1 I Y 9 A$ & $1 C M I A$ \\
\hline $2 Q B U A$ & $1 D 4 M 2$ & $1 M P S M$ & $2 H L E A$ & $1 G P 6 A$ & $1 G P 7 A$ & $3 E D T B$ & $2 Q D Y A$ \\
\hline $1 S R O A$ & $1 P S K L$ & $2 \mathrm{~V} 6 O A$ & $3 B Q V A$ & $2 A W G A$ & $2 E O O A$ & $2 H H E B$ & $1 B J G A$ \\
\hline $2 A A 2 A$ & $2 E C N A$ & $1 O U B A$ & 3EANA & $2 E X Y A$ & $3 D C T A$ & $1 T O Z A$ & $1 B Z Z A$ \\
\hline $1 J 3 D A$ & $2 J I D A$ & $2 P 2 C A$ & $2 E C U A$ & $3 H 02 A$ & $3 D J N B$ & $1 A J J A$ & $1 F L J A$ \\
\hline $2 F Y 9 A$ & $2 O 3 F A$ & $2 Y S S B$ & $2 D 3 E A$ & $2 O Z P A$ & $3 E R Z A$ & $1 C I C A$ & $2 A F 5 A$ \\
\hline $1 Q T M A$ & $1 N E K D$ & $1 U N 8 A$ & $1 B H Y A$ & $2 D F E A$ & $1 C N K A$ & $1 U V Q A$ & $1 B F T A$ \\
\hline $2 J P B A$ & $1 A Q A A$ & $1 \mathrm{CHVS}$ & $2 B U R B$ & $3 H M U A$ & $2 J X B A$ & $3 E G 7 A$ & $1 F T S A$ \\
\hline $2 N Q A A$ & $1 D O T A$ & $2 J 41 A$ & $1 C J C A$ & $1 U 07 A$ & $1 Z G K A$ & $1 W 4 H A$ & $1 K 1 A A$ \\
\hline $1 B 4 S A$ & $1 B Q 0 A$ & $1 J Y M A$ & $2 I T 6 A$ & $2 I B A A$ & $2 J 8 M A$ & $2 P 9 Y A$ & $3 C O V A$ \\
\hline $2 E K 7 A$ & $1 Q N 2 A$ & $2 P W Z A$ & $1 \mathrm{H} 2 \mathrm{VZ}$ & $1 U U E A$ & $1 Y I V A$ & $2 B E M A$ & $3 G 9 Y A$ \\
\hline 1OSEA & $1 K N Q A$ & $1 Y G S A$ & $2 A R J B$ & $1 V Z Y A$ & $1 H W 3 A$ & $3 G N L A$ & $2 Q J X A$ \\
\hline $2 W I O A$ & $1 Q X O A$ & $1 B W 9 A$ & $2 K 6 W A$ & $2 J X M B$ & $1 L 4 Z A$ & $1 L O 0 L$ & $3 E O 0 A$ \\
\hline $2 Z R E A$ & $2 F 1 E A$ & $1 V I O A$ & $1 E I A A$ & $1 C S 3 A$ & $1 A T P E$ & $2 D V W B$ & $2 G T N A$ \\
\hline $2 A Z H A$ & $1 J N L L$ & $2 Z 8 W C$ & $1 H 1 H A$ & $2 E S B A$ & $1 Q Z 8 A$ & $1 J W I B$ & $1 P P J D$ \\
\hline $1 Z T 7 A$ & $1 G T I A$ & $2 D 0 S A$ & $1 M E Y C$ & $1 I 3 D A$ & $1 X 3 S A$ & $1 Y N L H$ & $1 O G W A$ \\
\hline $1 K H U D$ & $1 Q 6 U A$ & $2 H 8 F A$ & $1 F D D A$ & $1 D V A L$ & $1 H I 6 A$ & $1 A H P A$ & $1 O A K L$ \\
\hline $1 D 5 B B$ & $1 W U I S$ & $1 B C 8 C$ & $3 B 8 K A$ & $1 P J Z A$ & $2 A 7 V A$ & $1 Q F K L$ & $1 M Y 7 A$ \\
\hline $2 H 3 L A$ & $1 Y 69 K$ & $2 P I L A$ & $2 B J Y A$ & $1 H K 0 X$ & $1 J O 0 A$ & $1 N B U A$ & $2 P 5 M A$ \\
\hline $2 I F F Y$ & $1 R D B A$ & $1 N D U A$ & $1 C P J A$ & $1 G X 3 A$ & $3 H M F A$ & 2PPTA & $1 I V 0 A$ \\
\hline $1 R 3 R A$ & $3 E 8 J A$ & $1 D I 2 A$ & $3 D G V A$ & $2 Y W L A$ & $1 Y 6 W A$ & $1 Z C 3 A$ & $1 Q G H A$ \\
\hline $2 D G T A$ & $1 D R B A$ & $1 W A 5 A$ & $1 F S E A$ & $2 R A 3 A$ & $2 H H O B$ & $2 S P O A$ & $1 U D 7 A$ \\
\hline $1 W 54 A$ & $1 F F T B$ & $1 Y Z G A$ & $1 A E 1 A$ & $1 B X W A$ & $1 K 82 A$ & $3 B J F A$ & $3 B R P A$ \\
\hline $1 A D 9 B$ & $1 B L U A$ & $1 Q C N A$ & $1 M Q O A$ & $1 G 79 A$ & $1 I Y U A$ & $3 G 7 L A$ & $2 J 65 A$ \\
\hline $3 C 90 X$ & $2 G 9 M A$ & $\widetilde{1 O P C A}$ & $1 N G G A$ & $1 F Y 7 A$ & $3 D 5 K A$ & $1 K 5 V A$ & $1 G 4 U R$ \\
\hline $2 C H 7 A$ & $2 A 3 V A$ & $2 D J 7 A$ & $1 D 7 A A$ & $2 R I P A$ & $2 N U 8 A$ & $1 M U 2 A$ & $2 A A B L$ \\
\hline $2 D D Y A$ & $1 Z V 8 A$ & $1 Z O V A$ & $1 D X Q A$ & $9 P A I A$ & $2 B S 3 A$ & $1 D 9 Z A$ & $1 D R 8 A$ \\
\hline $1 C G H A$ & $1 O P H A$ & $1 U 9 L A$ & $2 E Y 2 A$ & $2 B V 5 A$ & $3 C B 0 A$ & $3 E Y M A$ & $2 N L L B$ \\
\hline $1 C 6 S A$ & $2 Q W T A$ & $1 F O R L$ & $1 Z B A 1$ & $2 D L U A$ & $1 D K K A$ & $1 O I 7 A$ & $3 G P D R$ \\
\hline $2 D B Y A$ & $\widetilde{1 B L L E}$ & $2 F Q 3 A$ & $2 V 4 J C$ & $2 P P Y A$ & $2 O X B A$ & $1 A V H A$ & $1 E G 5 A$ \\
\hline $2 V 7 H A$ & $1 R U E 1$ & $2 O X F A$ & $2 V 4 O A$ & $2 C K F B$ & $1 U F Y A$ & $1 D 4 Z A$ & $1 E 4 X I$ \\
\hline $2 J F N A$ & $1 T 46 A$ & $2 Q 81 A$ & $1 D O 1 A$ & $1 D E 0 A$ & $1 F X L A$ & $1 K 25 A$ & $3 H 4 J A$ \\
\hline $1 Q D U A$ & $2 D L H A$ & $2 O 5 X H$ & 2ENPA & $1 J B A A$ & $1 E V T C$ & $1 A B 0 A$ & $1 M Q I A$ \\
\hline $1 Y P H E$ & $1 Y N W A$ & $3 B W T A$ & $1 H 1 Z A$ & $1 R V J L$ & $3 B Q D A$ & $2 E K X A$ & $2 Y V 4 A$ \\
\hline $1 B W V A$ & $1 H N A A$ & $1 B 1 B A$ & $1 G Q 8 A$ & $1 H K O A$ & $1 C H 9 A$ & $1 N M E B$ & $1 T F B A$ \\
\hline $2 A 83 A$ & $2 Y Q D A$ & $1 R C I A$ & $2 E M L A$ & $1 K Q F A$ & $1 R D 5 A$ & $3 A 0 I X$ & $3 H 43 A$ \\
\hline $1 H X 0 A$ & $2 V R 6 A$ & $1 J Y 0 A$ & $1 Z 7 W A$ & $2 N N Y A$ & $1 F I G L$ & $1 G G W A$ & $1 V Y D A$ \\
\hline $1 Z M 6 A$ & $1 A 0 N B$ & $1 D V I A$ & $1 U I 8 A$ & $1 L K Y B$ & $2 G M I A$ & $3 E X B A$ & $1 Z P E A$ \\
\hline $2 N M V B$ & $2 R 8 S L$ & $2 P 2 X A$ & $1 J G 8 A$ & $1 E Q 4 A$ & $1 P W K A$ & $2 O P 2 A$ & $1 Y S 4 A$ \\
\hline $1 G W O A$ & $5 C H Y A$ & $3 B W U D$ & $2 Z V 3 A$ & $3 B O 9 A$ & $2 R 31 A$ & $2 F 2 E A$ & 1 ROEA \\
\hline $2 J X W A$ & $2 N T F A$ & $2 K C X A$ & $1 H C H A$ & $1 H Y R B$ & $1 F B I H$ & $3 E 0 O A$ & $2 C 7 L B$ \\
\hline $1 C E E A$ & $2 U Y O A$ & $2 F O L A$ & $1 E 21 A$ & $2 I W 9 A$ & $1 J 36 A$ & $1 J 7 M A$ & $3 B Z 6 A$ \\
\hline $1 A A B A$ & $1 C 30 B$ & $1 C 1 J A$ & $2 D J B A$ & $1 Y M A A$ & $1 P I Z A$ & $1 N S U A$ & $1 E B D C$ \\
\hline $1 K Z L A$ & $1 E U G A$ & $1 O 6 L A$ & $1 S L M A$ & $2 E P Z A$ & $1 A 14 L$ & $2 K 9 N A$ & $1 E W 2 A$ \\
\hline
\end{tabular}


9.3 Appendix C: PDB enteries of training protein MSAs

Table 9.1 Pdb enteries of training MSAs - continued from previous page

\begin{tabular}{|c|c|c|c|c|c|c|c|}
\hline $1 I 3 O B$ & $2 C J 3 A$ & $1 C R W G$ & $1 N 2 S A$ & $2 C Z 8 A$ & $1 I 8 K B$ & $1 T C W A$ & $2 D W W A$ \\
\hline $2 M E B A$ & $2 Q 20 A$ & $1 S H M B$ & $1 B M G A$ & $1 Y I B A$ & $3 D O 6 A$ & $1 Y F S A$ & $1 K I 1 A$ \\
\hline $1 A U U A$ & $1 R 8 Q A$ & $2 F 1 V A$ & $1 T W 0 A$ & $1 W O H A$ & $1 V 67 A$ & $1 Z A A C$ & $3 D 4 D A$ \\
\hline $3 C R R A$ & $3 D X 6 A$ & $1 M J X A$ & $1 N G 6 A$ & $3 B S Z L$ & $1 K 4 Q A$ & $2 M C G 1$ & $1 V Z 0 A$ \\
\hline $2 V W S A$ & $3 B C Q A$ & $1 N N 7 A$ & $2 D 3 A A$ & $2 Y V 1 A$ & $1 W E S A$ & $2 A 77 L$ & $2 V D 8 A$ \\
\hline $1 P S 8 A$ & $1 B X 0 A$ & $2 C W 2 A$ & $2 V X P A$ & $2 Q M C B$ & $1 Q 41 A$ & $1 B 3 R A$ & $3 E N S A$ \\
\hline $1 F 9 M A$ & $2 C 7 W A$ & $2 Y W W A$ & $2 G H J A$ & $1 E A H 4$ & $1 Q O J A$ & $1 H 5 Q A$ & $2 E 3 H A$ \\
\hline $1 I J Y A$ & $2 I M 5 A$ & $2 O S S A$ & $3 C G 0 A$ & $1 Z 9 D A$ & $1 U J 0 A$ & $1 F D 9 A$ & $3 B A 7 A$ \\
\hline $1 Q P D A$ & $1 F D Q A$ & $2 E L I A$ & $1 Y V F A$ & $3 B K 8 A$ & $1 G Y C A$ & $1 H L K A$ & $1 H A X B$ \\
\hline $1 T A H A$ & $1 T E 0 A$ & $1 J G L L$ & $2 E L Z A$ & $1 R 4 P A$ & $3 E 70 C$ & $2 Y U J A$ & $1 D O Z A$ \\
\hline $2 V R P B$ & $2 Q 6 G A$ & $1 O I D A$ & $1 A R 61$ & $3 B C O A$ & $1 B T Y A$ & $2 J 0 F A$ & $1 A B 6 A$ \\
\hline $1 I 7 Q A$ & $1 D X H A$ & $2 V 09 A$ & $2 Q H N A$ & $2 E S W A$ & $2 E 74 B$ & 9PAPA & $1 O A U L$ \\
\hline $1 K N O A$ & $3 B 8 E A$ & $1 K V 0 A$ & $1 T J R A$ & $2 B 1 K A$ & $1 V R P A$ & $1 Y U C A$ & $2 Q 5 W D$ \\
\hline $1 U W A A$ & $2 O S O A$ & $1 I 1 Z A$ & $2 C U 8 A$ & $1 S B 6 A$ & $2 J M M A$ & $1 L 0 H A$ & $1 R 5 P A$ \\
\hline $1 I 7 G A$ & $1 A I H A$ & $2 C 2 V C$ & $7 F D 1 A$ & $1 E 93 A$ & $2 P H C B$ & $3 B I G A$ & $1 D X U B$ \\
\hline $2 W 1 G A$ & $1 E S 1 A$ & $2 D 31 A$ & $3 B A 4 A$ & $3 G A 5 A$ & $2 R 751$ & $1 Z M U A$ & $1 H F S A$ \\
\hline $2 P 5 C A$ & $2 G T D A$ & $1 H 1 T A$ & $2 W G J A$ & $1 D Z R A$ & $2 H I 9 A$ & $2 V 7 A A$ & $1 C I A A$ \\
\hline $1 M J S A$ & $3 G F 4 A$ & $1 H R O A$ & $1 J X S A$ & $1 S 17 A$ & $2 Q O F A$ & $1 O 6 A A$ & $3 E M N X$ \\
\hline $2 D I 2 A$ & $1 O 1 \mathrm{JB}$ & $2 H H L A$ & $3 I Y 3 B$ & $2 O S 1 A$ & $1 B J J A$ & $1 L 7 T H$ & $1 T F 3 A$ \\
\hline $2 D K R A$ & $3 I 5 T A$ & $3 B O S A$ & $4 M D H A$ & $1 Q S 0 A$ & $2 G G M A$ & $3 C Z J A$ & $1 E E P A$ \\
\hline $966 C A$ & $1 B 89 A$ & $1 F 11 A$ & $2 B P Q A$ & 1JHEA & $3 I 6 V A$ & $3 G L 3 A$ & $1 J 1 L A$ \\
\hline $2 Z R U A$ & $2 H 8 F B$ & $3 G H Z A$ & $1 M Q 1 A$ & $1 X G Q B$ & $2 R I W A$ & $1 X 09 A$ & $1 R R P A$ \\
\hline $1 I O Q A$ & $1 R J 5 A$ & $1 F C S A$ & $1 L W 0 B$ & $2 H F 1 A$ & $2 O W D A$ & $1 F C Y A$ & $1 D W K A$ \\
\hline $3 B H 0 A$ & $3 H J W A$ & $2 P O I A$ & $1 X F R A$ & $1 Y F K A$ & $1 M X 2 A$ & $1 E V 5 A$ & 2EEHA \\
\hline $3 E W F A$ & $3 E 02 A$ & $1 B A 0 A$ & $3 H F M L$ & $1 D P J A$ & $2 C 0 R A$ & $2 D U W A$ & $1 E 6 U A$ \\
\hline $2 J B M A$ & $1 E V F A$ & $2 F W L A$ & $2 H G M A$ & $2 I G 2 H$ & $1 C 83 A$ & $2 G I M A$ & $1 F 62 A$ \\
\hline $1 N S K R$ & $3 F 4 L A$ & $1 C C 5 A$ & $1 V K 2 A$ & $2 G O U A$ & $1 R O V A$ & $1 P Y O A$ & $1 D F 1 A$ \\
\hline $1 Q 0 X H$ & $2 F S 2 A$ & $1 I B E A$ & $2 C 1 N A$ & $1 C T 2 I$ & $2 Z 10 A$ & $1 M H L A$ & $2 I 3 H A$ \\
\hline $1 D 3 W A$ & $3 C T Q A$ & $1 J S T B$ & $2 J Q H A$ & $1 H 9 S B$ & $1 F 6 N L$ & $1 Y S 9 A$ & $2 J N V A$ \\
\hline $2 A 6 H C$ & $1 H F P A$ & $2 Z O M A$ & $3 \mathrm{C} 5 \mathrm{JB}$ & $8 A T C B$ & $2 Q 3 B A$ & $1 V P C A$ & $1 H J 5 A$ \\
\hline $1 C 1 B B$ & $1 R W 7 A$ & $3 E P Y A$ & $1 C F P A$ & $1 Z C N A$ & $1 I X 8 A$ & $1 E 4 E A$ & $1 \mathrm{COV} 4$ \\
\hline $1 X M 5 A$ & $2 B 1 X B$ & $3 E Z P A$ & $2 Z U Q C$ & $1 Y Z L A$ & $1 I X 9 A$ & $2 D M L A$ & $1 A T L A$ \\
\hline $2 O Q 3 A$ & $2 D Z T A$ & $2 P O V A$ & $3 B F 0 A$ & $1 X T T A$ & $3 B S X A$ & $3 B L M A$ & $1 U 4 J A$ \\
\hline $1 O A Q L$ & $1 Q M 0 A$ & $2 B U W B$ & $1 T 47 A$ & $2 A 0 L C$ & $2 O B 4 A$ & $1 S U P A$ & $2 V G G A$ \\
\hline $6 C C P A$ & $3 D H X A$ & $1 Q G 4 A$ & $1 K T Q A$ & $2 J 5 P A$ & $2 W 9 D H$ & $1 Y 4 A I$ & $3 B Q U D$ \\
\hline $1 F 00 I$ & $1 P B 1 A$ & $2 D 1 X A$ & $1 A B O A$ & $3 B K W A$ & $3 D 09 A$ & $1 K 2 F A$ & $2 I C 9 A$ \\
\hline $1 E W L A$ & $2 G P 6 A$ & $1 M H Y D$ & $3 F I F A$ & $1 P 52 A$ & $2 C 4 M A$ & $1 G P 2 G$ & $2 R 9 S A$ \\
\hline $2 E M H A$ & $2 V J 3 A$ & $1 C M Y B$ & $2 W 2 G A$ & $3 B 2 N A$ & $1 R D O 1$ & $1 C V A A$ & $1 P 3 J A$ \\
\hline $1 Q S F A$ & $2 V H 1 A$ & $2 D P N A$ & $1 R S R A$ & $2 A T X A$ & $1 K J 3 H$ & $1 A U W A$ & $2 A 02 A$ \\
\hline $1 M H S A$ & $1 A S 0 A$ & $2 R G P A$ & $1 I R 9 A$ & $1 V 11 A$ & $3 G L 5 A$ & $3 C H V A$ & $1 P U Z A$ \\
\hline $1 M B E A$ & $3 D H Q A$ & $1 Z L 3 A$ & $1 M U T A$ & $1 N I 5 A$ & $1 G F J A$ & $3 H H 1 A$ & $1 H B A B$ \\
\hline $1 F O D 4$ & $1 U I E A$ & $3 F L 0 A$ & $1 C B 9 A$ & $2 F 7 L A$ & $2 A 7 S A$ & $1 C S Y A$ & $2 N S H A$ \\
\hline $1 Z U X A$ & $1 F 4 H A$ & $1 F 6 L L$ & $2 D M O A$ & $1 I 9 E A$ & $2 V G 5 A$ & $1 X E C A$ & $2 B J D A$ \\
\hline $1 A 9 E A$ & $3 C 00 B$ & $6 F A B L$ & $2 R 4 F A$ & $1 G X S B$ & 1PMRA & $1 L O F D$ & $2 A 66 A$ \\
\hline $1 B F 3 A$ & $1 X 0 M A$ & $2 D K F A$ & $2 D 4 V A$ & $1 S Q N A$ & $1 N D H A$ & $1 Q V 6 A$ & $3 E 64 A$ \\
\hline $1 U S 5 A$ & $1 K K T A$ & $1 K O L A$ & $1 D S X A$ & $2 P T J A$ & $1 Q 2 X A$ & $1 N C Q B$ & $1 E K 6 A$ \\
\hline $1 E Z O A$ & $1 S 2 J A$ & $1 R T T A$ & $2 B O 1 A$ & $1 Y C 7 A$ & $2 R N 2 A$ & $3 C Y Y A$ & $3 D R M A$ \\
\hline
\end{tabular}


Table 9.1 Pdb enteries of training MSAs - continued from previous page

\begin{tabular}{|c|c|c|c|c|c|c|c|}
\hline $3 B Z T A$ & $1 F R F S$ & $2 G F Y A$ & $2 H 1 O A$ & $1 G Z 3 A$ & $3 B 5 J A$ & $1 E X 2 A$ & $1 O 6 D A$ \\
\hline $2 N X I A$ & $2 P C P A$ & $1 L P Q A$ & $1 A 9 Z A$ & $3 B 3 F A$ & $3 D F G A$ & $3 E P D 3$ & $2 D J 1 A$ \\
\hline $1 S U 0 B$ & $1 L 7 I L$ & $1 N D 4 A$ & $1 H V D A$ & $3 C Z C A$ & $2 G 9 H A$ & $2 H 0 P A$ & $2 Y X W A$ \\
\hline $3 I Y 5 A$ & $1 O X N A$ & $2 O 3 K A$ & $1 X 5 S A$ & $1 U E X C$ & $1 X H F A$ & $1 D T S A$ & $1 Y N J D$ \\
\hline $3 R S P A$ & $2 Q 1 Z A$ & $2 G D N A$ & $1 I P 7 A$ & $2 Q O 9 A$ & $2 D K S A$ & $1 H J R A$ & $2 J 4 W D$ \\
\hline $3 D 54 B$ & $3 C W 0 A$ & $1 V J W A$ & $1 J L A A$ & $1 M I Q A$ & $1 M 04 A$ & $3 B D M B$ & $1 T X A A$ \\
\hline $3 B O W A$ & $1 J W Q A$ & $1 U T X A$ & $2 H 75 A$ & $1 B K M A$ & $2 A X 9 A$ & $3 F R 4 A$ & $2 B I M A$ \\
\hline $1 V I I A$ & $2 R N J A$ & $1 A K N A$ & $1 L A J A$ & $1 F 05 A$ & $2 B L F A$ & $1 B I I A$ & $2 D L Y A$ \\
\hline $1 W M V A$ & $1 J J E A$ & $2 C F Y A$ & $2 I S 8 A$ & $1 D O S A$ & $1 H V A A$ & $2 Q 07 A$ & $1 I A O B$ \\
\hline $1 N I 2 A$ & $1 V H U A$ & $1 U 06 A$ & $1 U M R C$ & $1 L 1 D A$ & $1 C T L A$ & $1 M Z A A$ & $3 E S 3 A$ \\
\hline $1 J Y S A$ & $1 O S F A$ & $1 L 9 Q A$ & $2 I T V A$ & $1 E G J H$ & $1 X Y 0 A$ & $2 E 54 A$ & $1 E U J A$ \\
\hline $2 E Y 5 A$ & $2 N V 6 A$ & $1 U D O A$ & $1 J G Y M$ & $1 Z 91 A$ & $1 Z H C A$ & $1 U G B A$ & $2 Q X C A$ \\
\hline $1 R 57 \mathrm{~A}$ & $2 I O V A$ & $1 S 96 A$ & $2 Q E 0 A$ & $1 H J 8 A$ & 2RJTA & $1 U U L A$ & $1 M 57 C$ \\
\hline $2 D 2 Q A$ & $1 N T G A$ & $3 E M O C$ & $2 P F 0 A$ & $2 C 6 N A$ & $1 T 1 T A$ & $1 L I 9 A$ & $1 A Y Z A$ \\
\hline $2 D A 2 A$ & $1 J C A A$ & $2 K J K A$ & $1 I B D A$ & $1 V Z U A$ & $2 K 91 B$ & $3 D K 7 A$ & $1 F 91 A$ \\
\hline $2 E O I A$ & $2 B 1 Z A$ & $1 D I 0 A$ & $1 Z 8 R A$ & $2 I 4 Q A$ & $3 C 5 W A$ & $1 D D 4 C$ & $2 D N P A$ \\
\hline $1 I G J A$ & $1 Y P 1 A$ & $2 R H Z A$ & $1 F 8 E A$ & $3 C F 6 R$ & $1 F T C A$ & $2 P S Y A$ & $2 O 2 C A$ \\
\hline $1 Y 0 S A$ & $1 U L N A$ & $1 E K S A$ & $2 N L J C$ & $1 B K L A$ & $4 T M K A$ & $2 U Z 9 A$ & $2 T G I A$ \\
\hline $3 E K O A$ & $1 K A 9 H$ & $1 E G W A$ & $2 B O Y A$ & $1 F 3 O A$ & $4 U B P B$ & $2 A Q Q A$ & $2 O X 4 A$ \\
\hline $2 I 7 G A$ & 2OGIA & 2ENIA & $2 \mathrm{ORBH}$ & $1 G N G A$ & $1 E A 5 A$ & $2 Q 6 N A$ & $2 A 7 R A$ \\
\hline $1 W H 3 A$ & $1 Z Y S A$ & $2 N L 8 A$ & $1 D P E A$ & $2 E 2 A A$ & $2 Z Q Q A$ & $3 E T P A$ & $2 N S 1 B$ \\
\hline $1 F V C A$ & $2 Q 3 R A$ & $1 P O U A$ & $3 B U 2 A$ & $2 T B S A$ & $2 P 3 U B$ & $2 A F P A$ & $1 Q B Z A$ \\
\hline $1 D 2 A A$ & $1 Q Q D B$ & $3 G 5 L A$ & $2 V U L A$ & $2 C T 2 A$ & $1 M Z R A$ & $1 X 3 X A$ & $1 H I L B$ \\
\hline $2 V S L A$ & $1 T 2 M A$ & $3 C L N A$ & $1 A A M A$ & $3 D 4 M A$ & $1 R V T H$ & $1 F 1 A A$ & $1 S N N A$ \\
\hline $3 D D 4 A$ & 3DPRE & $1 N Y O A$ & $1 G 2 S A$ & $2 K C P A$ & $2 C X 4 A$ & $1 K N 1 A$ & $3 E U B 2$ \\
\hline $1 A 5 M C$ & $1 M 2 I A$ & $2 Q W O B$ & $3 E 3 B X$ & $3 F G H A$ & $1 Y N Y A$ & $1 W 5 C F$ & $2 H K Y A$ \\
\hline $2 G F W A$ & $3 C 9 W A$ & $2 B J M H$ & $1 D 0 Q A$ & $1 N 3 B A$ & $2 V C Q A$ & $1 Z V V W$ & $1 H I X A$ \\
\hline $1 I 2 A A$ & $3 D G E A$ & $1 Q H H D$ & $2 F M K A$ & $1 I 5 S A$ & $3 D 57 A$ & $1 K J Q A$ & $1 L K J A$ \\
\hline $1 P 8 D A$ & $3 D 66 A$ & $1 E 28 A$ & $1 M A 3 A$ & $1 G H 4 A$ & $1 U H C A$ & $2 W G M A$ & $2 C 0 D A$ \\
\hline $2 P 4 B A$ & $3 C P I G$ & $2 D X S A$ & $2 B 0 L A$ & $2 F T N A$ & $3 H C S A$ & $1 H 85 A$ & $2 H F P A$ \\
\hline $2 R M L A$ & $1 M R E L$ & $2 C C S A$ & $1 A V 8 A$ & $2 D A O A$ & $1 U E G A$ & $1 R L 2 A$ & $2 G N C A$ \\
\hline $1 F 66 D$ & $1 Q 8 I A$ & $2 J P C A$ & $2 Z 43 A$ & $3 E 2 Y A$ & $1 G 16 A$ & $1 F L 5 A$ & $1 G 5 Z A$ \\
\hline $2 A W T A$ & $1 J R 9 A$ & $1 O D 6 A$ & $1 O 7 \mathrm{JA}$ & $1 I 5 D A$ & $2 A 1 W H$ & $1 Y Y H A$ & $2 H C 8 A$ \\
\hline $1 W E J H$ & $2 Q J R A$ & $1 E G A A$ & $2 Q G U A$ & $1 C V E A$ & $1 G E J A$ & $2 Q E 7 D$ & $1 H 6 K Z$ \\
\hline $1 A S G A$ & 1JUDA & $1 Y N I A$ & $1 Q 98 A$ & $1 X G U B$ & $1 B O 0 A$ & 2JEJA & $1 O 78 \mathrm{~A}$ \\
\hline $1 M 3 K A$ & $2 F M J A$ & $1 U W 9 A$ & $2 K L 5 A$ & $1 D 8 Y A$ & $3 D U U A$ & $8 P R K A$ & $2 F 2 B A$ \\
\hline $1 P 8 P A$ & $1 P O H A$ & $1 Z 3 N A$ & $1 Q F W L$ & $5 С P A A$ & $2 H W 0 A$ & $2 Y X R A$ & $1 C E 2 A$ \\
\hline $1 P Y T C$ & $1 Q W D A$ & $1 L 8 Q A$ & $3 B W 4 A$ & $2 W A 8 A$ & $3 E G V B$ & $3 B A 1 A$ & $2 P 8 W S$ \\
\hline $2 R G N C$ & $3 C 4 C A$ & $2 W G X A$ & $1 P 2 H A$ & $1 N U C A$ & $1 J B O B$ & $1 L J Y A$ & $2 D K 2 A$ \\
\hline $3 F T T A$ & $1 P K D A$ & $1 Z N C A$ & $1 P Y C A$ & $2 J K 6 A$ & $2 G 0 F A$ & $3 H E I A$ & $1 D N 0 A$ \\
\hline $1 O Y 5 A$ & $3 H O F A$ & $1 Q S 0 B$ & $1 H 9 Q A$ & $3 B 9 Y A$ & $1 X 0 A A$ & $1 F W G C$ & $1 G 8 O A$ \\
\hline $1 P V C 1$ & $1 P R 3 A$ & $1 O 12 A$ & $2 O T G A$ & $2 E D Y A$ & $3 P M G A$ & $1 F U E A$ & $2 D 4 A B$ \\
\hline $2 G R X C$ & $1 C D O A$ & $2 D T 1 A$ & $1 Z 8 P A$ & $3 G 4 D A$ & $1 J T O A$ & $3 B E W A$ & $3 I Y 3 A$ \\
\hline $1 L Q W A$ & $3 I Y 2 B$ & $1 P 4 L B$ & $2 K 48 A$ & $1 T 2 K A$ & $1 B U P A$ & $1 A 4 I A$ & \\
\hline
\end{tabular}


9.4 Appendix D: Curriculum Vitae 


\section{Curriculum Vitae}

\section{PERSÖNLICHE DATEN}

Name:

Geburtstag:

Geburtsort:

Familienstand:

Staatsangehörigkeit

Abschluss:
Mehmet Gültas

17.05.1984

Kirikkale/Türkei

Ledig

Türkisch

Promotion

\section{AUSBILDUNG}

09/1998-06/2002

09/2002-06/ 2006

09/2006-09/2007

10/2007-10/2009

10/2009-06/2013

Seit 01.07.2013
Kirikkale Anadolu Lisesi (Gymnasium), Türkei, Abschluss Abitur.

Studium der Statistik und Computer Science an der Karadeniz Technische Universität, mit Abschluss B.Sc.

Erfolgreicher Abschluss der Deutschen Sprachprüfung.

Studium der Angewandten Informatik mit dem Nebenfach Bioinformatik an der Georg-August Universität Göttingen, mit Abschluss M.Sc.

Doktorand und wissenschaftlicher Mitarbeiter im Institut für Informatik an der Georg-August Universität Göttingen, mit Abschluss Promotion.

Wissenschaftlicher Mitarbeiter im Institut für Bioinformatik an der Universitätsmedizin, Georg-August Universität Göttingen. 


\section{BERUFSERFAHRUNG}

03/2008-11/2008

02/2009-06/2009

$10 / 2008-11 / 2009$

Seit 01.11.2009
Studentische Hilfskraft bei der Fakultät für Geowissenschaften und Geographie, Georg-August Universität Göttingen.

Studentische Hilfskraft bei der Fakultät für Geo- und Umweltwissenschaften, Ludwig-Maximilians Universität München.

Studentische Hilfskraft beim Deutschen Forschungszentrum für Luft- und Raumfahrt.

Wissenschaftliche Betreung sowohl unterschiedliche Vorlesungen als auch Bachelor, Master oder Forschungsorientierte Projektarbeiten.

\section{BESONDERE KENNTNISSE}

Sprachkenntnisse:

PC-Kenntnisse:

Sonstiges:
Türkisch (Muttersprache), Deutsch - fliessend in Wort und Schrift, Gute Englisch Kenntnisse.

Fundierte Programmierungs-Kenntnisse, insbesondere Java, PHP, Sql, Html, JavaScript, Css und Flash.

Langjährige Tätigkeit in der Selbstverwaltung des Studentenwohnheims sowie Tätigkeit als Vorsitzender. 


\section{PUBLIKATIONEN}

[1] Erpenbeck D, Voigt O, Gültas M, Wörheide G: The sponge genetree server-providing a phylogenetic backbone for poriferan evolutionary studies. Zootaxa 2008,1939:58-60.

[2] Körner MC, Schöbel A, Ashauer K, Schulz K, Gieshold M, Rex R, Zhang H, Gültas M: Book Chapter: Projekt Promotoranalyse in Gene Graphen, Organismen; Modellierungsund Anaysemethoden in der Systembiologie Shaker Verlag 2010.

[3] Gültas M, Haubrock M, Tüysüz N, Waack S: Coupled Mutation Finder: A new entropybased method quantifying phylogenetic noise for the detection of compensatory mutations. BMC Bioinformatics 2012, 13:225.

[4] Gültas M, Düzgün G, Herzog S, Jäger SJ, Meckbach C, Wingender E, Waack S: Quantum Coupled Mutation Finder: Predicting functionally or structurally important sites in proteins using quantum Jensen-Shannon divergence and CUDA programming. BMC Bioinformatics 2014, 15:96.

[5] Dong Z, Wang K, Gültas M, Welter M, Dang L, Wierschin T, Stanke M, Waack S: CRFbased models of protein surfaces improve protein-protein interaction site predictions. BMC Bioinformatics 2014, under review.

[6] Gültas M, Haubrock M, Wlochowitz D, Zeidler S, Waack S, Wingender E: TF-Spiker: A novel information theory-based method for the identification of functionally important transcription factors in co-expressed genes. Bioinformatics 2014, under review.

\section{Conferences, Workshops, Meetings}

- Statistical and dynamical models in biology and medicine (October, 2011, Göttingen): Poster präsentation

- Workshop über Algorithmen und Komplexität, 63. Theorietag (Januar, 2012, Brandennburg): Vortag über "A new entropy-based method quantifying phylogenetic noise for the detection of compensatory mutations"

- German Conference of Bioinformatics (September, 2012, Jena): Poster präsentation

- Meeting Gene Regulation and Information Theory (April, 2013, Halle): Poster präsentation

- German Conference of Bioinformatics (September, 2013, Göttingen): Poster präsentation 